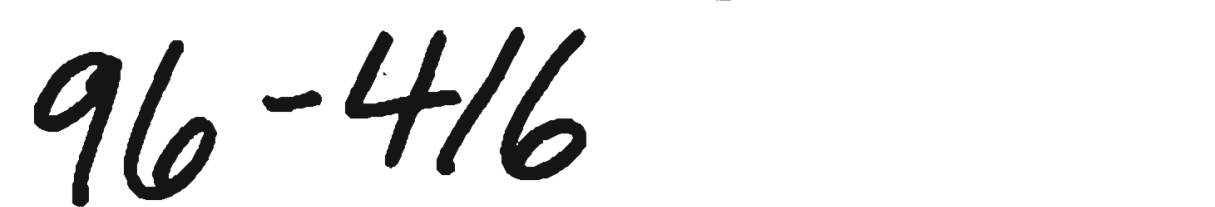

Ground-Water and Surface-Water Elevations in the

University of Alaska Fairbanks Area, 1992-95

U.S. GEOLOGICAL SURVEY

Open-File Report 96-416

Prepared in cooperation with the

UNIVERSITY OF ALASKA FAIRBANKS 
|

I

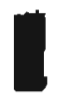

I

I

I

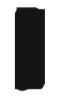




\section{Ground-Water and Surface-Water Elevations in the University of Alaska Fairbanks Area, 1992-95}

By Melanie L. Jackson and Michael R. Lilly

U.S. GEOLOGICAL SURVEY

Open-File Report 96-416

Prepared in cooperation with the

UNIVERSITY OF ALASKA FAIRBANKS

Fairbanks, Alaska 1996 


\section{U.S. DEPARTMENT OF THE INTERIOR \\ BRUCE BABBITT, Secretary}

\section{U.S. GEOLOGICAL SURVEY}

Gordon P. Eaton, Director

Special thanks to Anne Kriegler, David Claar, and Sonja Benson for their assistance in the production of this report.

For additional information write to:

District Chief

U.S. Geological Survey 4230 University Drive, Suite 201 Anchorage, AK 99508-4664
Copies of this report can be purchased from:

U.S. Geologlcal Survey

Branch of Information Services

Box 25286

Denver, CO 80225-0286

http//Boatdakanc.wr.usgs.gov/index.html 


\section{CONTENTS}

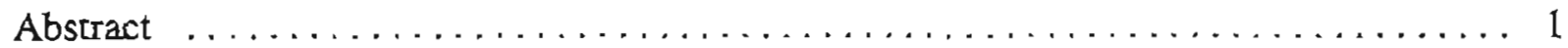

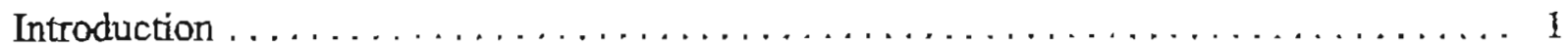

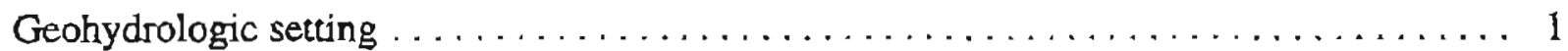

Ground-water sites. . . . . . . . . . . . . . . . . . . . . . . . . . 5

Surface-water sites. . . . . . . . . . . . . . . . . . . . . . . . . 10

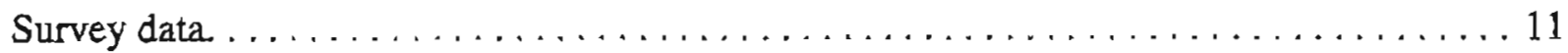

Ground-water and surface-water elevations $\ldots \ldots \ldots \ldots \ldots \ldots \ldots \ldots \ldots \ldots \ldots \ldots$

References cited. . . . . . . . . . . . . . . . . . . . . . . . . . . . . 11

Water-elevation tables and hydrographs $\ldots \ldots \ldots \ldots \ldots \ldots \ldots \ldots \ldots \ldots$

FIGURES

1. Map showing the location of Fairbanks, Alaska, and the University of Alaska Fairbanks study area. . . . . . . . . . . . . . . . . . . . . . 2

2. Map showing the University of Alaska Fairbanks study area, with hydrologic data-collection sites $\ldots \ldots \ldots \ldots \ldots \ldots \ldots \ldots \ldots \ldots \ldots \ldots \ldots \ldots$

3. Stage hydrographs of the Chena River at Fairbanks gaging station and the Tanana River at Fairbanks gaging station for calendar years 1991 to $1995 \ldots \ldots \ldots \ldots \ldots 4$

4. Map showing locations of hydrologic data-collection sites near the intersection of Fairbanks Street and Geist Road in the University of Alaska Fairbanks study area . 6

5. Diagram showing derivation of local numbers from the official rectangular subdivision of public lands.

\section{TABLES}

1. Ground-water data collection sites, University of Alaska Fairbanks area . . . . . . 7

2. Surface-water data-collection sites, University of Alaska Fairbanks area . . . . . . 10

CONVERSION FACTOAS AND VERTICAL AND HORIZONTAL DATUMS

\begin{tabular}{rll}
\hline Muttiply & \multicolumn{1}{c}{ By } & To obtain \\
\hline fool $(\mathrm{ft})$ & 0.3048 & meter \\
mile $(\mathrm{mi})$ & 1.609 & kilometer \\
square mile $\left(\mathrm{mi}^{2}\right)$ & 2.59 & square kilometer \\
gallon (gal) & 3.785 & liter \\
gallons per minute (gai/min) & 0.06308 & liters per second \\
& & \\
\hline
\end{tabular}


Vertical Datum:

In this report, "sea leves" refers to the National Geodetic Vertical Daturn of 1929 (NGVD of 1929), a geodetic datum derived from a general adjustonent of the first-order level nets of both the United States and Canada, formerly called Sea Level Datum of 1929.

Horizontal Daturn:

The horizontal datum for all locations in this report is the North American Datum of 1927. Multiple reference marks were used from Alaska Departrnent of Transportation and Public Facilities surveys. Global positioning survey insoruments were also used to determine horizontal control points. 


\title{
Ground-Water and Surface-Water Elevations in the University of Alaska Fairbanks Area, 1992-95
}

\author{
By Melanie L. Jackson and Michael R. Lilly
}

\begin{abstract}
The U.S. Geological Survey, in cooperation with the University of Alaska Fairbanks, collected water-elevation data at 50 ground-water sites and 11 surface-water sites. These data were collected during 1992 to 1995 to characterize the geohydrology of the University of Alaska Fairbanks area.
\end{abstract}

\section{INTRODUCTION}

The U.S. Geological Survey (USGS), in cooperation with the University of Alaska Fairbanks (UAF), is presently (1996) conducting a cooperative study to characterize the geohydrology of the UAF area, which lies west of and adjacent to the city of Fairbanks (figs. 1 and 2). The UAF study area is about $3 \mathrm{mi}^{2}$ in size and lies partly in the alluvial plain of the Chena and Tanana Rivers and partly on College Hill. University Avenue forms the eastern boundary of the study area, with the Chena River on the south, and the Parks Highway on the west (figs. 1 and 2). College Hill, where the main UAF campus is located, forms the northern boundary of the study area (fig. 2).

Water levels in 50 ground-water wells and at 11 surface-water sites were measured at monthly intervals. In addition, water levels at selected sites were measured at more frequent intervals to monitor short-term changes in ground-water levels caused by rapid stage changes of the Chena and Tanana Rivers. Additional water levels were measured to document the effects of UAF water-supply well pumping on the ground-water system. Ground-water data are maintained in the USGS Ground-Water Site Inventory (GWSI) data base and surface-water data are maintained in the USGS Automatic Data Acquisition and Processing System (ADAPS) data base. This report presents ground-water and surface-water elevation data collected between May 1992 and October 1995.

\section{GEOHYDROLOGIC SETTING}

The UAF campus is in the Tanana River Valley on the northem boundary between the alluvial sand and gravel deposits of the Chena Alluvium (Péwé and others, 1976) and the bedrock hills of the Yukon-Tanana Upland (Anderson, 1970). The primary depositional source of the Chena Alluvium is the Tanana River. Sediment facies within the Chena Alluvium are laterally discontinuous, as is typical of deposits from braided rivers (Rust, 1978). The thickness of these deposits is unknown but may be in excess of $500 \mathrm{ft}$ in places (Nelson, 1978). The bedrock hills are part of a metamorphic rock system that forms the Yukon-Tanana Upland. Most wells in the bedrock obtain water from fracture systems that have formed during numerous tectonic events (Nelson, 1978). 
Eolian silts of the Fairbanks Loess and reworked silt deposits of the Ready Bullion Formation cover the bedrock hills and grade into alluvial-fan silt deposits and the Chena Alluvium.

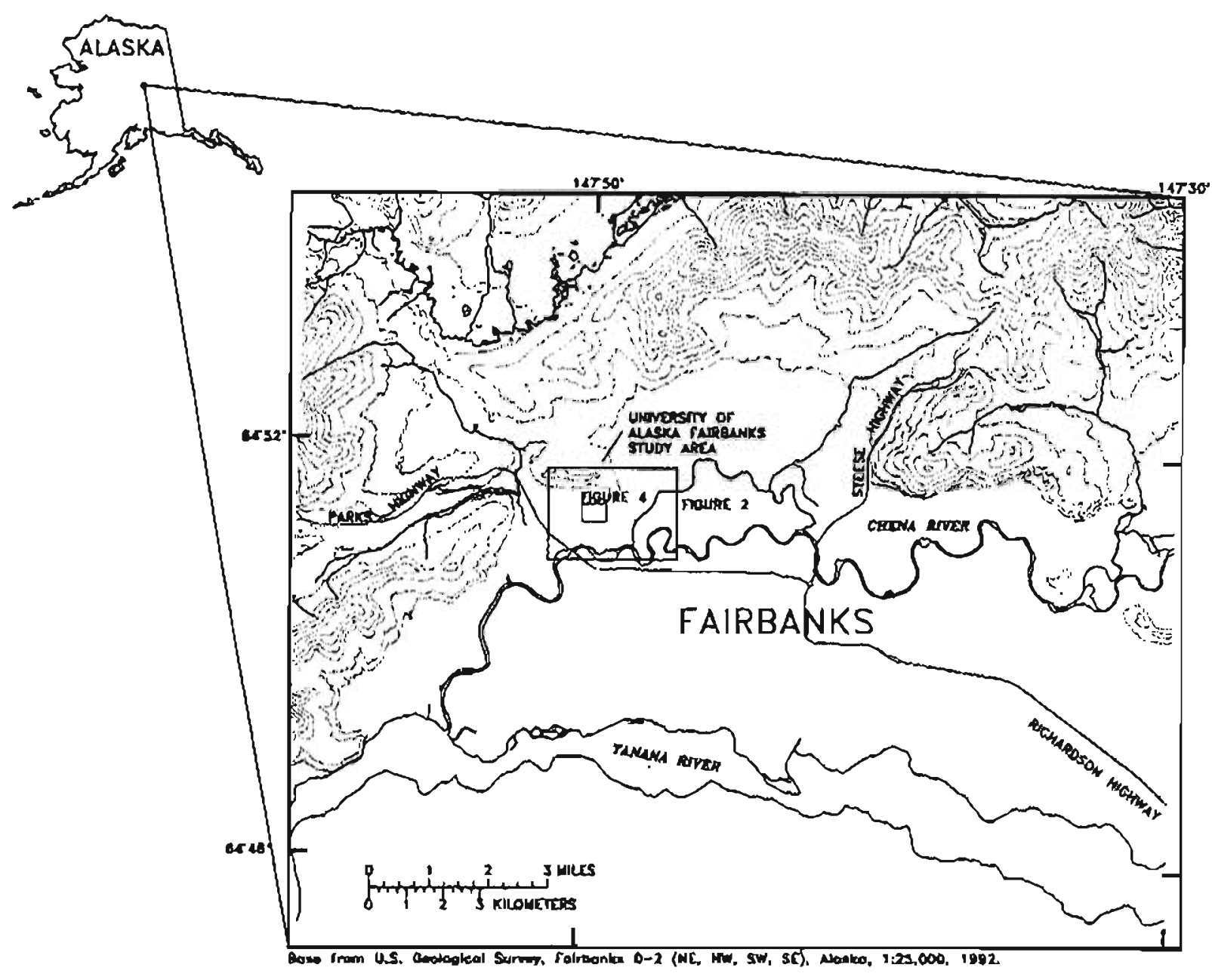

Figure 1. Location of Fairbanks, Alaska, and the University of Alaska Fairbanks study area.

The distribution of permafrost in the study area is discontinuous. Geotechnical borings west of the study area at the Parks Highway and Geist Road intersection indicate that permafrost extends from approximately $40 \mathrm{ft}$ below the land surface down to depths greater than $100 \mathrm{ft}$ below the land surface (Alaska Department of Transportation and Public Facilities, 1990). Drillers' logs of wells throughout the study area indicate that the permafrost thickness ranges from 5 to more than $30 \mathrm{ft}$. UAF water-supply wells located between College Hill and Geist Road are in permafrost-free zones. Péwé and Bell (1974) describe the permafrost distribution in the study area and characterize the ground as containing a moderate to high ice content.

The Tanana River is the main surface-water influence on ground water in the Fairbanks area alluvial aquifer (Nelson, 1978; Glass and others, in press). Typically, the Tanana River stage rises for one to two weeks during the spring, as a result of snowmelt runoff at lower elevations and icejam effects during breakup. A prominent stage increase usually occurs for a longer period during the middle of the surnmer, in response to snow and glacial runoff from the Alaska Range, $50 \mathrm{mi}$ 


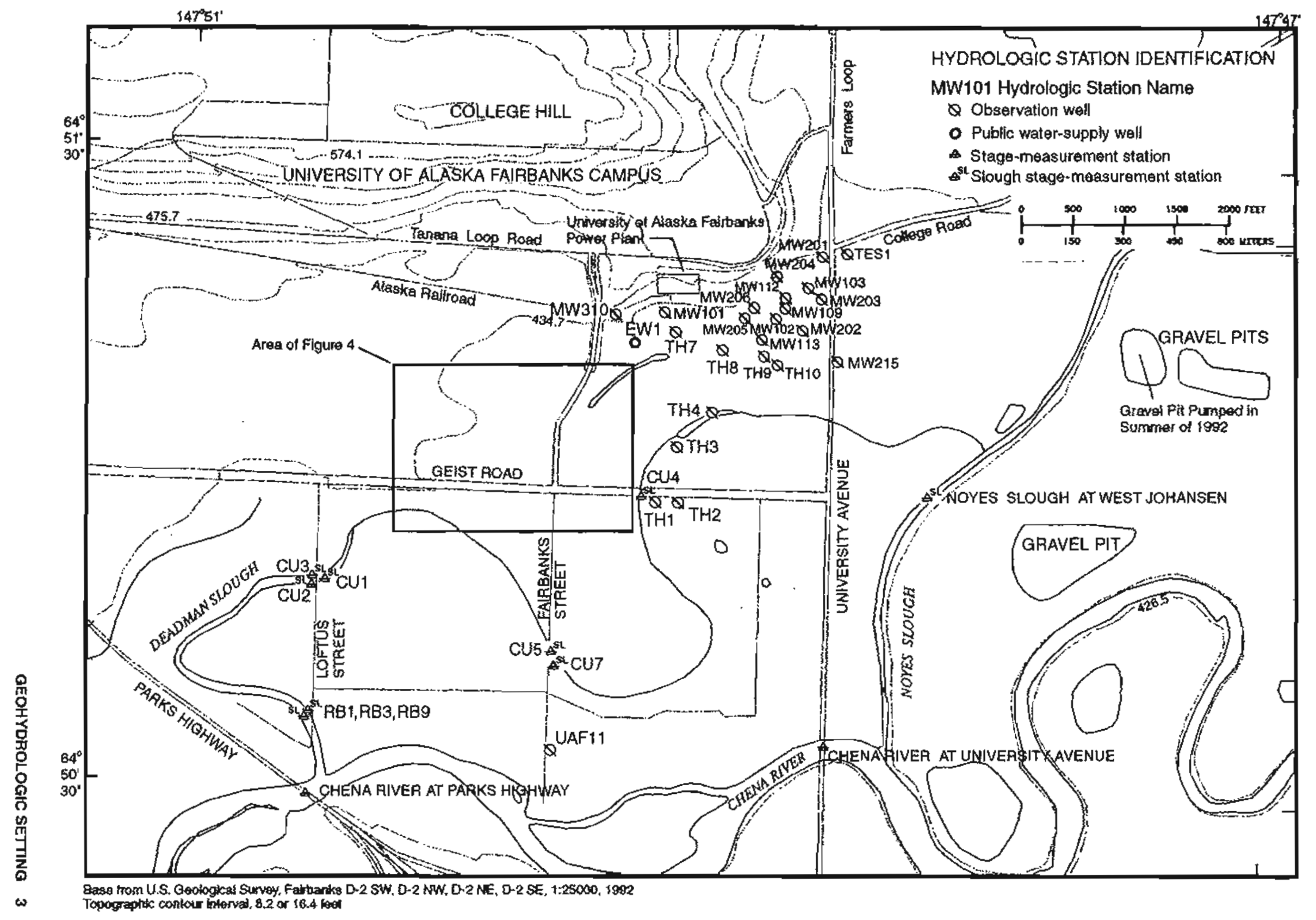

Figure 2. Unlversity of Alaska Fairbanks study area, with hydrologic data-collection sltes. 
south of the area shown in figure 2, or from excessive rainfall in the Yukon-Tanana Upland (fig. 3). The Tanana River stage decreases during late sumuer when temperatures drop in the Alaska Range and high-elevation snow and glacial runoff declines. The stage levels increase after a complete ice layer covers the river, as a result of an increase in flow resistance from the ice cover. Stage levels then decrease throughout the winter because of a continuous reduction in flow (fig. 3).
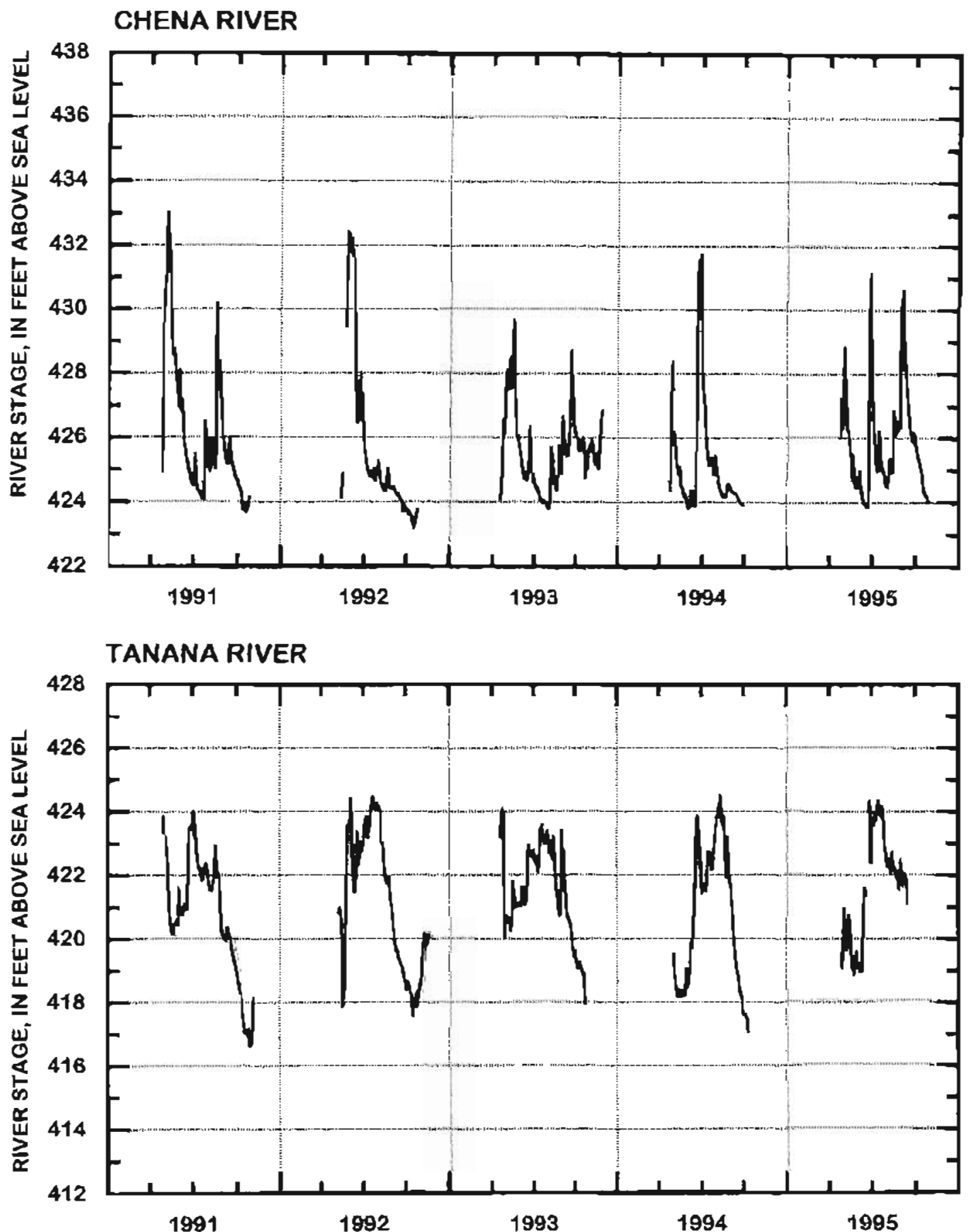

Figure 3. Stage hydrographs of the Chena River at Fairbanks gaging station and the Tanana River at Fairbanks gaging station for calendar years,1991 to 1995. 
Backwater from the Tanana River as well as variation in flow in the Chena River affects the Chena River stage within the study area. Flow in the Chena River rises during spring snowmeltrunoff and late-summer rainfall-runoff events. Ice also covers most of the Chena Rjver during the winter season, and stages are lower during this time (fig. 3). Deadman Slough enters the Chena River just upstream from the Parks Highway bridge (fig. 2). The culverts at crossings of Deadman Slough under Loftus Street, Fairbanks Street, and Geist Road control the flow through the slough (fig. 2). Backwater effects from the Chena River extend up the slough to the crossing at Geist Road. Water-level data-collection sites north of Geist Road and just south of the Fairbanks Street Bridge are in old slough channels leading to Deadman Slough but are no longer connected to Deadman Slough. Sediments of the Chena Alluvium lie beneath the silty slough deposits.

The Chena River at Fairbanks continuous-record gaging station is located about 0.5 mi downstream from the Steese Highway bridge; the Tanana River at Fairbanks continuous-record gaging station is about $5 \mathrm{mi}$ southwest of the UAF main campus (fig. 1). Data from these sites, located outside the UAF study area, are included for comparison with the hydrographs at the back of this report.

The depth from land surface to ground water is less than $20 \mathrm{ft}$ throughout most of the study area. Ground-water levels respond to changes in stage of the Chena and Tanana Rivers. Pumping at a gravel pit to the east of the study area during the summer of 1992 (fig. 3) caused a drop in ground-water levels during the summer of 1992 and early winter of 1993 . The water levels in observation wells during the 1993 and 1994 summer seasons are probably more typical of normal levels.

UAF has two primary water-supply wells (GW JB and GW2) located to the northwest of the Geist Road and Fairbanks Street intersection (fig. 4). A third water-supply well (EW1), located to the south of the UAF power plant and east of the Fairbanks Street Alaska Railroad overpass (fig. 2), provides an emergency back-up water supply. Observation wells are located throughout the study area (figs. 2 and 4). Most of the wells were installed for previous environmental site investigations. Additional observation wells, installed near the primary water-supply wells during the summer and early winter of 1992 and 1994, were used to understand water-supply well pumping effects.

\section{GROUND-WATER SITES}

During this study, water levels were measured in a total of 50 wells (figs. 2, 4, and table 1). Most of the wells are relatively shallow-less than $30 \mathrm{ft}$ deep-and screened across the water table in the Chena Alluvium. The water elevation in a well was determined by using either a steel tape and chalk or an electric tape to measure the vertical distance between a measuring point and the water surface. The electric tapes were calibrated against steel tapes and correction factors were applied. Replicate measurements were taken at each well until depth-to-water readings were duplicated within $0.01 \mathrm{ft}$ for measurements made by steel tapes and within $0.02 \mathrm{ft}$ for those made by electric tapes. The measuring points at all wells used in this study were labeled to avoid errors. 


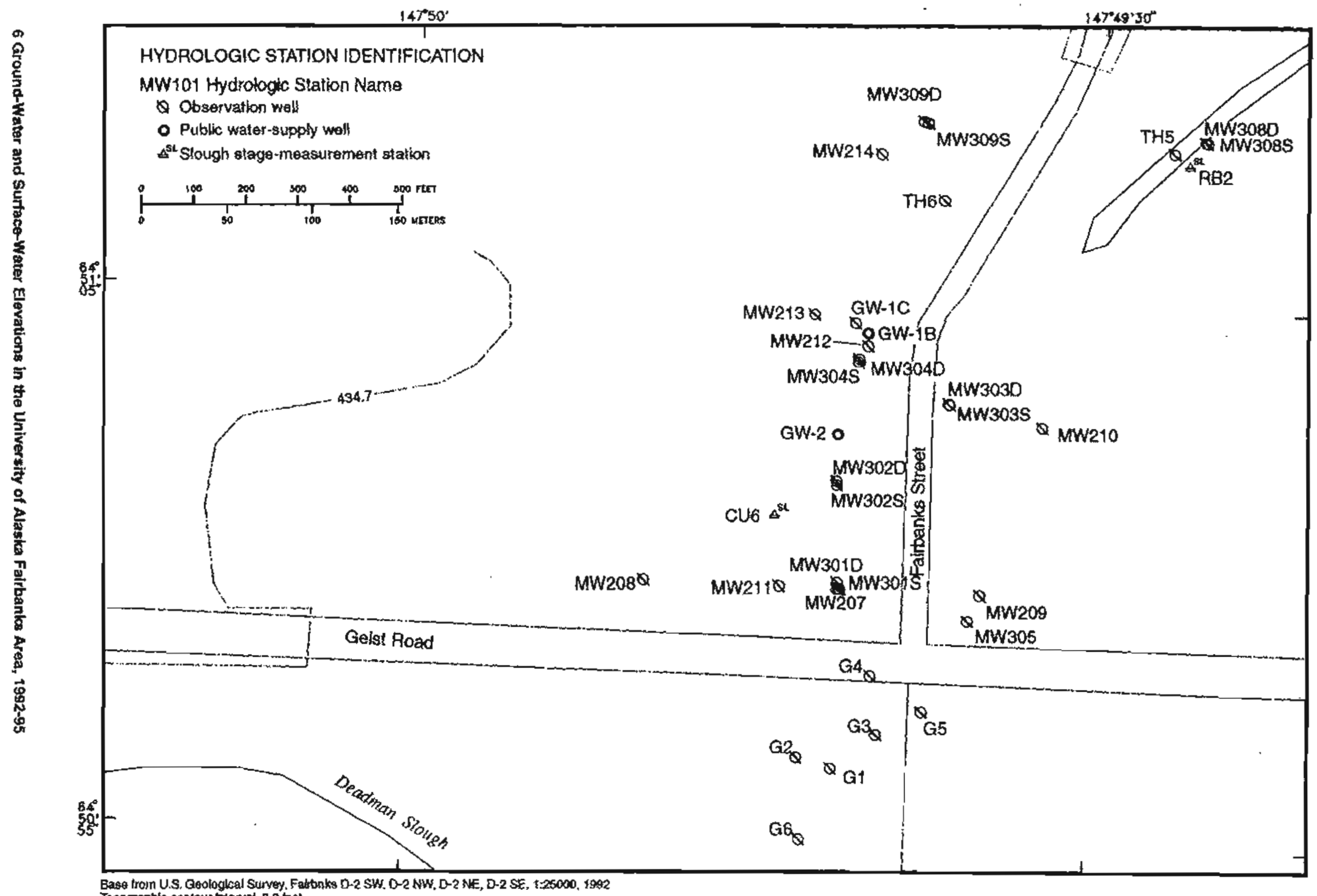

Easa Iron U.S, Goologlcal Survey, Fabtoks O-2 SW. D-2 WW, D-2 NKE, D-2 SE, t-26000, 1992

Figure 4. Locations of hydrologic data-colfection sites near the intersection of Fairbanks Street and Geist Road in the University of Alaska Fairbanks study area. 
Table 1. Ground-water data-collection sites, University of Alaska Fairbanks area

\begin{tabular}{|c|c|c|}
\hline USGS Field ID & GWSI Site ID & GWSI Local Number \\
\hline EW1 & 645113147492101 & PCO0100106DBCB 1 \\
\hline G5 & 645056147493601 & FC00100107BAAA I \\
\hline G6 & 645054147494301 & FC00100107BAAB 1 \\
\hline GW1B & 645104147494002 & FCO0100106CDDB I \\
\hline GWIC & 645104147494004 & FCO0100106CDDB2 \\
\hline GW2 & 645102147494102 & FCO0100106CDDB3 \\
\hline MW101 & 645112147491601 & FC00100106DBCAl \\
\hline MW102 & 645115147485001 & FCO0100106DACA1 \\
\hline MW103 & 645117147484301 & FC00100106DABD2 \\
\hline MW109 & 645116147484801 & FC00100106DACA2 \\
\hline MW112 & 645117147484801 & FCO0100106DACA3 \\
\hline MW113 & 645113147485301 & FCO0100106DACC1 \\
\hline MW201 & 645121147484001 & FC00100106DAAB 1 \\
\hline MW202 & 645114147484401 & FC00100106DACA4 \\
\hline MW203 & 645117147484001 & FCO0100106DADB 1 \\
\hline MW204 & 645119147485001 & FCO0100106DABD 1 \\
\hline MW20S & 645115147485701 & FC00100106DBDAl \\
\hline MW206 & 645116147485501 & FCO0100106DACB 1 \\
\hline MW207 & 645059147484101 & FCO0100106CDDCS \\
\hline MW208 & 645059147494901 & FC00100106CDCD 1 \\
\hline MW209 & 645059147493401 & FC00100106CDDD 1 \\
\hline MW210 & 645102147493301 & FC00100106CDDA 1 \\
\hline MW211 & 645059147494501 & FC00100106CDCD2 \\
\hline$M W 212$ & 645103147494001 & FCO0100106CDDB4 \\
\hline MW213 & 645104147494501 & FC00100106CDCA1 \\
\hline$M W 214$ & 645108147494601 & FC00100106CDBA1 \\
\hline MW215 & 645111147483601 & FCO0100105CBCC1 \\
\hline MW301D & 645059147494001 & FCO0 $100106 C D D C 2$ \\
\hline MW301S & 645059147494002 & FCO0100106CDDC3 \\
\hline
\end{tabular}


Table 1. Ground-water data-collection sites, University of Alaska Fairbanks area (Continued)

\begin{tabular}{|c|c|c|}
\hline USGS Feld ID & GWSI Site ID & GWSI Local Number \\
\hline$M W 302 D$ & 645101147494001 & FCOO100106CDDC4 \\
\hline MW302S & 645101147494002 & FCO0100106CDDC5 \\
\hline MW304D & 645103147494001 & FC00100106CDDBS \\
\hline MW304S & 645103147494002 & FC00100106CDDB6 \\
\hline MW308D & 645116147492601 & FCO0100106DCBAl \\
\hline MW3085 & 645116147492602 & FC00100101DCBA2 \\
\hline MW309D & 645109147494001 & FCO0100106CDAB 1 \\
\hline MW309S & 645109147494002 & FCO0100106CDAB2 \\
\hline MW310 & 645116147492601 & FCO0100106DBCB 1 \\
\hline TES ] & 645121147483501 & $F C 00100106 C B B B 1$ \\
\hline TH1 & 645057147491601 & FCO0100107ABBA1 \\
\hline TH2 & 645157147491001 & FCO0100107ABAB! \\
\hline TH3 & 645103147491101 & FCO0100106DCDBI \\
\hline TH4 & 645106147490401 & FC00100106DCAD1 \\
\hline TH5 & 645110147492901 & FC00100106CDAAI \\
\hline TH6 & 645107147493701 & FC00100106CDAC1 \\
\hline TH7 & 645113147491401 & FC00100106DBCD 1 \\
\hline $2 \mathrm{H} 8$ & 645112147490201 & FC00100106DBDD 1 \\
\hline TH9 & 645111147485201 & FC000100106DACC1 \\
\hline TH10 & 645115147484901 & FC00100106DDBAI \\
\hline UAF1! & 645036147493801 & FC00100107BDDA 1 \\
\hline
\end{tabular}

The USGS field identification number (field ID) for ground-water sites was assigned on the basis of previous projects and the University's usage of the site. The ground-water site identification (site $\mathrm{DD}$ ) is based on latitude and longitude and is the primary identifier used in the USGS data base GWSI. The local number is based on the State Rectangular Coordinate System, defined by base and meridian, township, range, section, and quarter section to the fourth order number (fig. 5). 


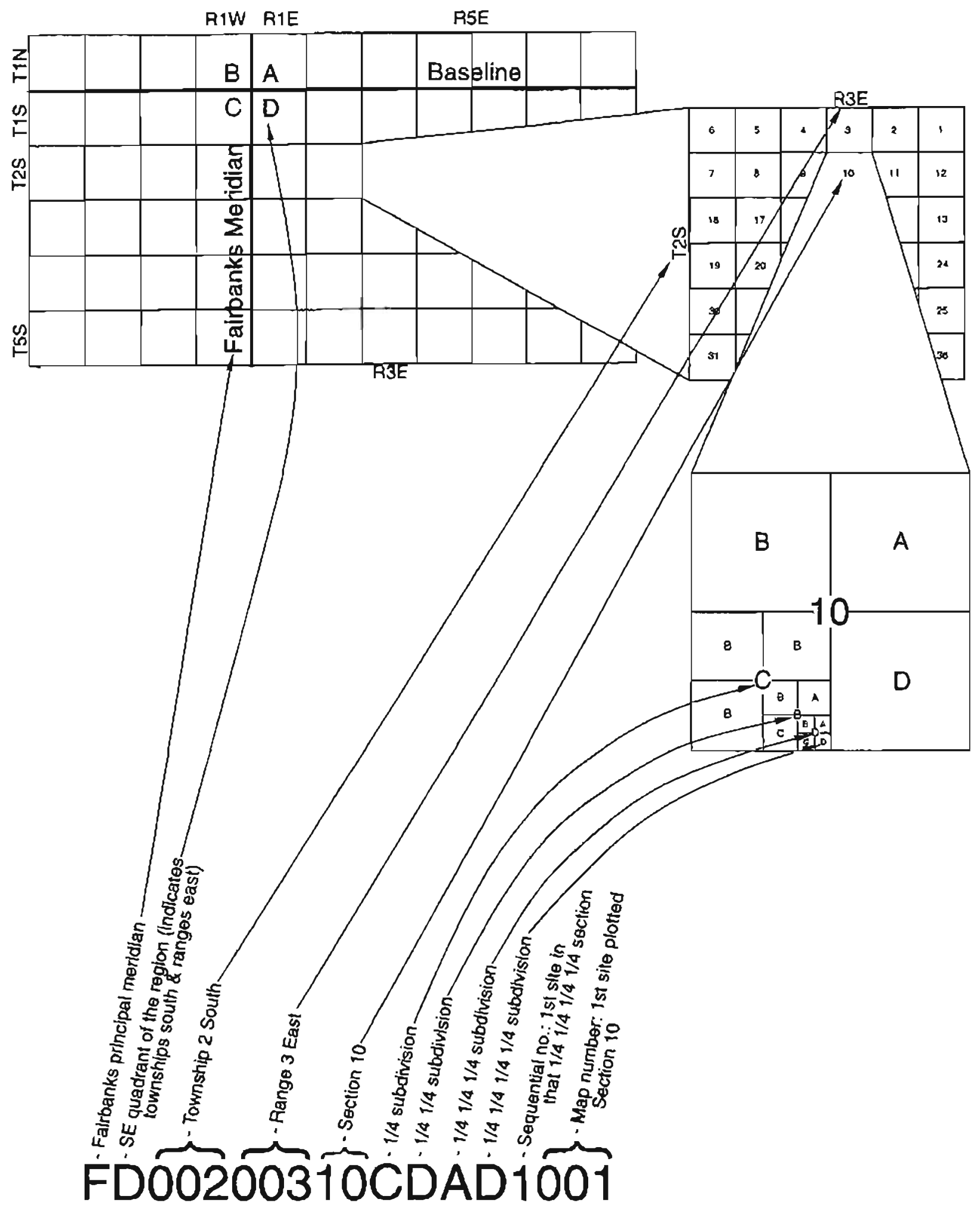

Figure 5. Derivation of local numbers from the official rectangular subdivision of public lands. 


\section{SURFACE-WATER SITES}

Water elevations were measured at 11 surface-water sites: 2 on the Chena River and 9 on miscellaneous sloughs (figs. 2, 4, and table 2). The USGS operates continuous-record gaging stations on both the Chena and Tanana Rivers. The sloughs act as retention ponds for snowmelt and rainfall runoff. Water elevations at most surface-water sites were determined by reading a staff gage or by using a measuring tape to obtain the distance from the water surface to a point of known elevation. At gaging-station sites, water elevations were determined by using wire-weight gages on bridges or by surveying to the water surface. In the winter, holes were cut through the ice to measure water elevations.

Table 2. Surface-water data-collection sites, University of Alaska Fairbanks area

\begin{tabular}{|c|c|}
\hline USGS Field ID & ADAPS Station Number \\
\hline \multicolumn{2}{|c|}{ Sloughs } \\
\hline CU1 & 1551401760 \\
\hline $\mathrm{CU} 2$ & 1551401760 \\
\hline CU3 & 1551401760 \\
\hline CU4 & 1551401710 \\
\hline CU5 & 1551401730 \\
\hline CU6 & 1551401710 \\
\hline CU7 & 1551401730 \\
\hline RBI & 1551401790 \\
\hline RB2 & 1551401730 \\
\hline \multicolumn{2}{|c|}{ Rivers } \\
\hline Chena River at University Avenue Bridge & 15514016 \\
\hline Chena River at Parks Highway Bridge & 15514018 \\
\hline
\end{tabular}

The USGS field identification number (field $\mathrm{DD}$ ) was assigned according to whether the site is a culvert site (CU) or a rebar site (RB), and then numbered chronologically according to the date of its first use. The surface-water station number consists of a two-digit number representing the state, followed by a six-digit number representing the downstream order number. The numbers are not consecutive, thus allowing for new sites to be added in intermediate locations. This information is included in the ADAPS data base. 


\section{SURVEY DATA}

Elevations of reference points at all water-elevation measuring sites in this study were surveyed to the National Geodetic Vertical Datum of 1929. This datum is called "sea level" in this report. Elevations of all reference points in this study were tied to a reference benchmark (University 2) located near the Duckering Building on the UAF campus. Its elevation is $492.36 \mathrm{ft}$ (U.S. Coast and Geodetic Survey, 1966). The elevations of reference points forming the UAF vertical survey net were connected to the vertical survey net used by the USGS in a study of the Fairbanks International Airport study area, located southwest of the UAF study area (Claar and Lilly, 1995), and to the vertical survey net used by the USGS in a study of the Alaska Railroad Industrial Area located east of the UAF study area (Kriegler and Lilly, 1995; Lilly and others, 1996).

Selected wells were re-surveyed to check and adjust elevations for frost jacking. Frost jacking occurs mainly in late spring or early summer, after the snow has melted. Updated measuring point elevations are applied to water elevations after the estimated time of movernent. Horizontal locations of sites were determined by horizontal ground surveys, satellite-based global positioning systems, and from maps of the study area. The horizontal datum for all locations in this report is North American Datum of 1927.

\section{GROUND-WATER AND SURFACE-WATER ELEVATIONS}

The remainder of this report consists of a section called "Water-Elevation Tables and Hydrographs." This section presents all the ground-water and surface-water data collected in the UAF area between 1992 and 1995. Data for each site consist of water elevations collected during mass measurements (MM), which are nearly synoptic sets of measurements made once each month at a large group of sites, and partial measurements (PM), which are sets of measurements made as needed at a smaller selection of sites.

Each table showing water levels contains information about the site, well construction, and each measurement made at the site. Land surface (LS) is the average elevation of the ground immediately surrounding the site. The measuring point (MP) is the point of known elevation from which all water-elevation measurements are taken. The error is the difference between replicate measurements made at each well during a given mass or partial measurement.

Hydrographs show all water elevations contained in the corresponding table. Each point on the hydrograph represents a measured water elevation. Uniform time and elevation scales for all hydrographs are used to allow direct comparison between hydrographs. Data points are plotted and connected with lines. These lines show the estimated trends between measurements; however, the connecting lines do not reflect any shorter period stage changes that may have occurred between measurements. No trend lines were drawn between points 2 months or more apart.

\section{REFERENCES CITED}

Alaska Department of Transportation and Public Facilities. 1990, Parks Highway/Chena Ridge inlerchange bridge no. 1878 \& 1879 (bridge no. 1879 future construction): Fairbanks, Alaska, unpublisbed report, $18 \mathrm{p}$.

Anderson, G.S., 1970, Hydrologic teconnaissance of the Tanana Basin, Central Alaska: U.S. Geological Survey Hydrologic Investigations Atlas HA-319, 4 sheets. 
Claar, D.V., and Lilly, M.R., 1995. Ground-water and surface-water elevations in the Fairbanks International Airport area, Alaska, 1990-94: U.S. Geological Survey Open-File Report 95-382.155 p.

Gilfilian Engineering, Inc., 1993, Groundwater characterization study, University Car Care Cencer: Aachorage, Alaska, prepared for the Alaska Department of Environmental Conservation. 27 p. +1 appendix [GEI project no. 192033).

Glass, R.L., Lilly, M.R., and Meyer, D.F., in press, Ground-water levels in an alluvial plain between the Tanana and Chena Rivers near Fairbanks, Alaska, 1986-93: U.S. Geological Survey Wacer-Resources Investigations Report 96-4060.

Kriegler, A.T., and Lilly, M.R, 1995, Water-elevation, stream-discharge, and ground-water quality datn in the Alaska Railroad Industrial Area, Fairbanks, Alaska, May 1993 to May 1995: U.S. Geological Survey Open-File Report 95-364, $247 \mathrm{p}$.

Lilly, M.R., McCarthy, K.A., Kriegler, A.T., Vohden, J., and Burno, G.E., 1996, Compilation and preliminary interpretations of hydrologic and water-quality daca from the Railroad Industrial Area, Fairbanks. Alaska, 1993-94: U.S. Geological Survey Water-Resources Investigations Report 96-4049, 45 p.

Nelson, G.L., 1978, Hydrologic information for land-use planning, Fairbanks vicinity, Alaska: U.S. Geological Survey Open-File Report 78-959, 47 p.

Péwé, T.L., and Bell. J.W., 1974, Map showing distribution of permafrost in the Fairbanks D-2 SW quadrangle, Alaska: U.S. Greological Survey Miscellaneous Investigations Series Map I-829-B, scale 1:24,000, 1 sheet.

Pewé, TL. Bell, J.W., Forbes, R.B., and Weber, F.R., 1976, Geologic map of the Fairbanks D-2 SW quadrangle, Alaska: U.S. Geological Survey Miscellaneous Investigations Series Map 1-829-A, scale 1:24000, 1 sheet.

Rust, B.R, 1978, Depositional models for braided alluvium, in Miall, A.D., ed., Fluvial sedimentology: Canadian Society of Petroleum Geology Memoir 5, p. 605 - 625.

U.S. Coast and Geodetic Survey, 1966, Vertical concrol datum: U.S. Department of Commerce, Environmental Sciences Services Administration, September, $26 \mathrm{p}$. 


\section{WATER-ELEVATION TABLES AND HYDROGRAPHS}


EW1 WATER-SUPPLY WELL

All measurements in feet Depth to bottom of well from MP: Depth from TOC to top of SI :

Depth from TOC to bottom of SI : Land Surface Dakum :

Datum corrections, reference survey notes in site folders Date $\mid$ MP elevation (feet above sea level) previously named GW3

Site 10: Local Number:

(1)

\begin{tabular}{c|c} 
Feet & Elevation \\
\hline 44.0 & 386.2 \\
30.0 & 400.2 \\
44.0 & 386.2 \\
& 428.5
\end{tabular}

\begin{tabular}{c|c} 
Date & MP elevation (feet above sea level) \\
\hline $02-21-95$ & 430.24
\end{tabular}

\begin{tabular}{|c|c|c|c|c|c|c|c|c|}
\hline Date & Time & Method & MP to WS & Error & LS to WS & WS stevation & Flow Rate (gavmin) & Remerks \\
\hline $12-08-93$ & 1618 & E-tape & $\cdot 11.90$ & 0.01 & 10.16 & $41 \overline{8.34}$ & NA & $P^{\prime} T$ \\
\hline $12-09-93$ & 1053 & Steel tape & 11.94 & 0.01 & 10.20 & 418.30 & NA & PT \\
\hline $12-09-93$ & 1342 & Steel tape & $\{1.94$ & 0.01 & 10.20 & 418.30 & NA & PT \\
\hline $12-09-93$ & 1337 & Steed tape & 14.25 & 0.01 & 12.51 & 415.99 & NA & $\mathrm{PT}$ \\
\hline $12-09-83$ & 1342 & Steel tape & 19.45 & 0.01 & 17.71 & 410.79 & NA & PT \\
\hline $12-09-93$ & 1711 & Steel tape & 19.90 & 0.01 & 18.16 & 410.34 & 300 & PT, $2233300 \mathrm{gad}$ \\
\hline $12-09-93$ & 1740 & Steel tape & 19.99 & 0.01 & 18.25 & 410.25 & 300 & PT, 2245000 gal \\
\hline $12-09-93$ & 1808 & Steed tape & 20.05 & 0.01 & 18.31 & $4+0.19$ & 300 & PT, $2252000 \mathrm{gal}$ \\
\hline $12-09-93$ & 2032 & Steel tape & 20.36 & 0.01 & $\uparrow 8.62$ & 409.88 & NA & $\mathrm{PT}$ \\
\hline $12-09-93$ & 2133 & Steel tape & 20.51 & 0.01 & 18.77 & 409.73 & NA & PT \\
\hline $12-09-93$ & $2 \uparrow 44$ & Steel tape & 12.35 & 0.04 & 10.61 & 417.89 & NA & PT \\
\hline $12-09-93$ & 2145 & Steel tape & 12.34 & 0.01 & 10.60 & 417.90 & NA & PT \\
\hline $12-09-93$ & 2151 & Steel tape & 12.34 & 0.01 & 10.60 & 417.90 & NA & PT \\
\hline $12-09-93$ & 2220 & Steel tape & 12.24 & 0.01 & 10.50 & 418.00 & NA & PT \\
\hline $12-10-93$ & 1446 & Steel tape & 11.94 & $0.0 t$ & 10.20 & 418.30 & NA & PT \\
\hline $02-21-95$ & 1315 & Steel tape & 13.49 & 0.01 & 11.75 & 416.75 & NA & PT \\
\hline $02-21-85$ & 1335 & Steel tape & 13.51 & 0.01 & 11.77 & 416.73 & NA & PT \\
\hline $02-22-95$ & 1049 & Steel tape & 13.54 & 0.01 & 11.80 & 416.70 & NA & PT \\
\hline $02-22-95$ & 1153 & Steel tape & 16.65 & 0.01 & 14.91 & 413.59 & NA & PT \\
\hline 02-24-95 & 1002 & Steel tape & 17.28 & 0.01 & 15.54 & 412.96 & $\mathrm{NA}$ & PT \\
\hline
\end{tabular}

LS, fand surface

MP, measuring point

$N A$, not available

$P M$, partial measurement

PT. pump test

SI, screened interval

$T O C$, top of casing

WS, water surface 
EW1 WATER-SUPPLY WELL previously named GW3
(Continued)

\begin{tabular}{|c|c|c|c|c|c|c|c|c|}
\hline Date & Time & Method & MP to WS & Error & LS to WS & WS elevation & Flow Rate (gal/min) & Remarks \\
\hline $02-27-95$ & 1540 & Steel tape & 17.76 & 0.01 & 16.02 & 412.48 & NA & PT \\
\hline $03-01-95$ & 0936 & Steel tape & 18.35 & 0.01 & 16.61 & 411.89 & NA & PT \\
\hline $03-02-95$ & 0856 & Steel tape & .17 .89 & 0.01 & 16.15 & $4\{2.35$ & NA & PT \\
\hline $03-06-95$ & 1040 & Steel tape & 17.79 & 0.01 & 16.05 & 412.45 & NA & PT \\
\hline $03-08-95$ & 1600 & Steet tape & 17.66 & 0.01 & 15.92 & 412.58 & NA & PT \\
\hline $03-10-95$ & 0953 & Steel tape & 17.80 & 0.01 & 16.06 & 412.44 & NA & PT \\
\hline 03-13-95 & 1158 & Steel tape & 17.87 & 0.01 & 16.13 & 412.37 & NA & PT \\
\hline $03-20-95$ & 1507 & Steel tape & $\uparrow 3.73$ & 0.01 & 11.98 & 416.51 & NA & PT \\
\hline $04-18-95$ & 0955 & Steel tape & 12.85 & 0.01 & 11.11 & 417.39 & NA & MM \\
\hline $07-18-95$ & 1036 & Steel tape & 10.76 & 0.01 & 9.02 & 419.48 & NA & MM \\
\hline $08-15-95$ & 0846 & Steel tape & 10.85 & 0.01 & 9.11 & 419.39 & NA & MM \\
\hline $08-16-95$ & 1103 & Steel tape & 10.83 & 0.01 & 9.09 & 419.41 & NA & MM \\
\hline $09-15-95$ & 1655 & Steel tape & 10.08 & 0.01 & 8.34 & 420.16 & NA & MM \\
\hline $10-17-95$ & $\$ 230$ & Stesl tape & 10.66 & 0.01 & 8.92 & 419.58 & NA & MM \\
\hline
\end{tabular}

Site ID:

645113147492101

Local Number: $\frac{\text { FC00100106DBCB1 }}{-}$

.




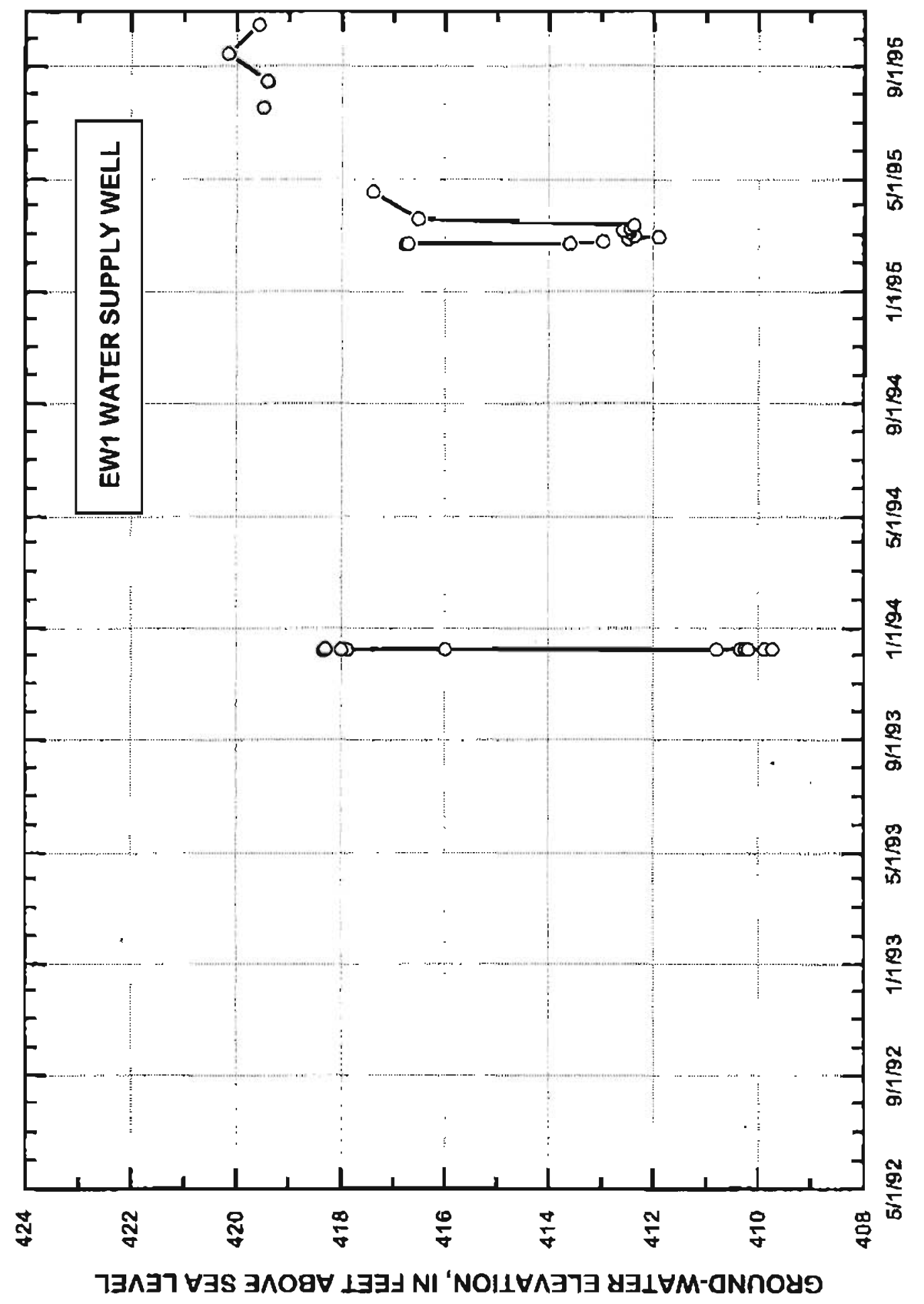




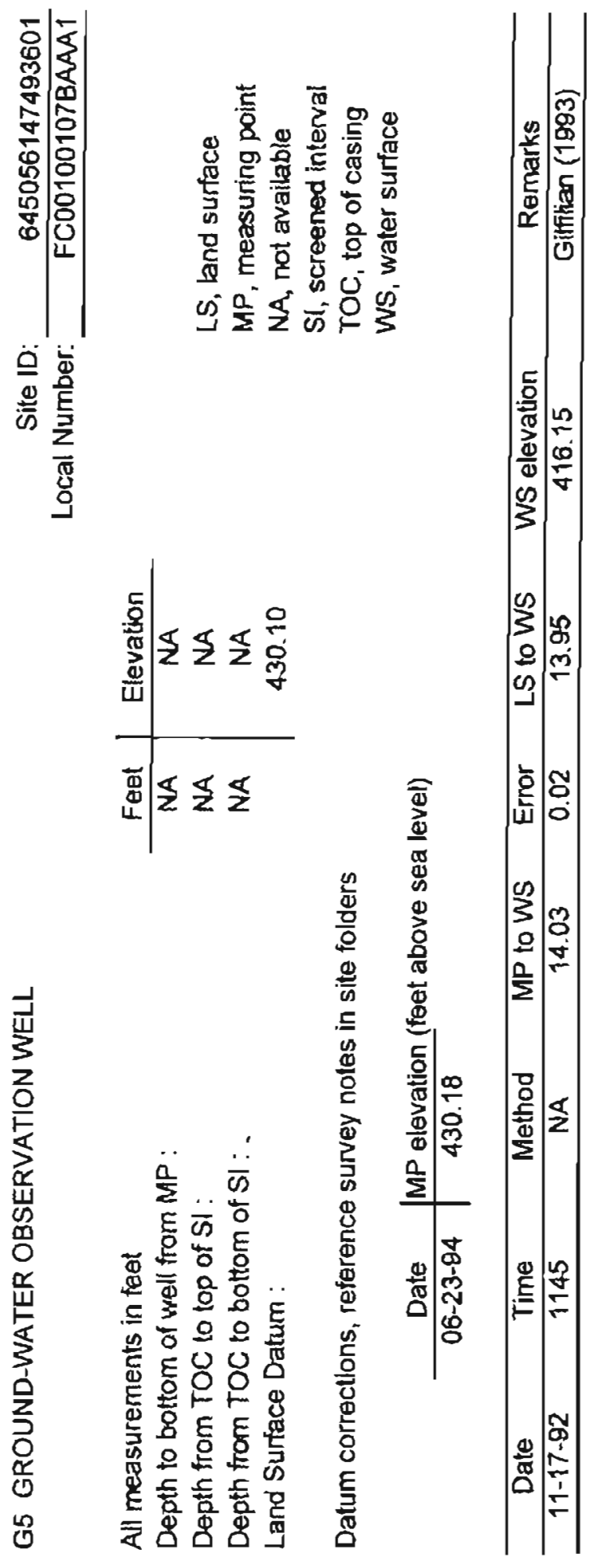




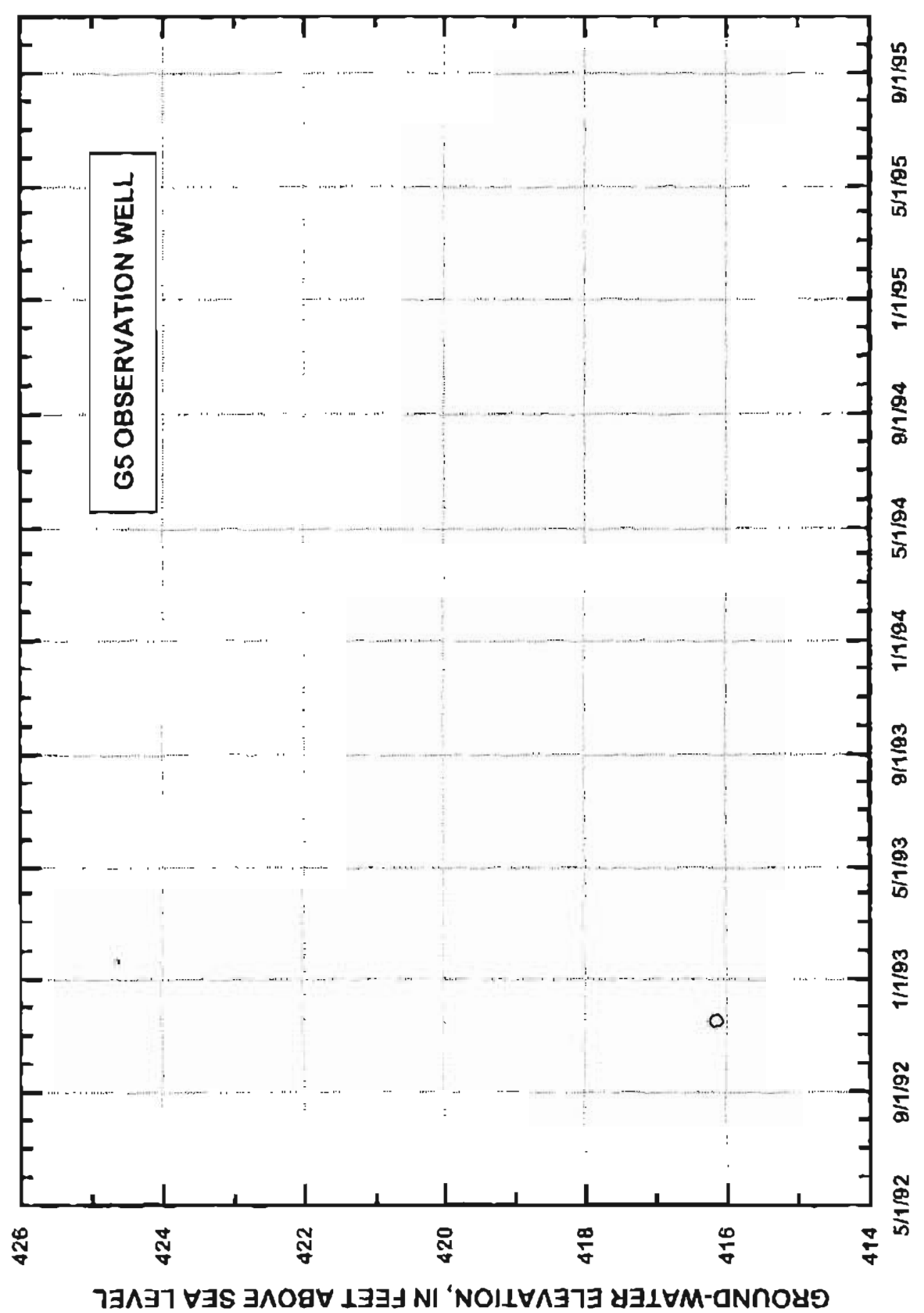


Site 10: Local Number

\begin{tabular}{c|c} 
Feet & Elevation \\
\hline 8.0 & 422.4 \\
18.0 & 412.4 \\
& 430.5
\end{tabular}

Alf meastrements in feet Depth from TOC to top of SI:

Depth from TOC to bottom of S1:

Land Surface Datum :

Datum corrections, reference survey notes in site folders

\begin{tabular}{c|c} 
Date & MP eievation (feet abova sea level) \\
\hline $8-17-93$ & 430.37
\end{tabular}
LS, land surtace MP, measuring point

NA, not available

$P M_{1}$ partial measurement SI, screened interval TOC, lop of casing

WS, water surface

\begin{tabular}{|c|c|c|c|c|c|c|c|}
\hline Date & Time & Method & MP to WS & Error & LS to WS & WS elevation & Remarks \\
\hline $11-18-92$ & $\mathrm{NA}$ & NA & 15.32 & 0.02 & 15.45 & $4 \longdiv { 5 . 0 5 }$ & Giffilan (1993) \\
\hline $02-24-93$ & 1600 & Steel tape & 13.05 & 0.01 & 13.18 & 417.32 & PM \\
\hline $02-25-93$ & 1310 & Steel tape & 13.06 & 0.01 & 13.19 & 417.31 & $M M$ \\
\hline $03-12-93$ & 1404 & Steel tape & 13.28 & 0.01 & 13.41 & 417.09 & MM \\
\hline $03-17-93$ & 1530 & Steel tape & 13.38 & 0.01 & 13.51 & 416.99 & $P M$ \\
\hline $04-09-93$ & 1150 & Steel tape & 13.52 & 0.01 & 13.65 & 416.85 & PM \\
\hline $04-16-93$ & 1530 & Sleel tape & 12.96 & 0.01 & 13.09 & 417.41 & $M A$ \\
\hline $05-18-93$ & 1528 & Steel tape & 10.39 & 0.01 & 10.52 & 419.98 & MM \\
\hline $06-11-93$ & 1011 & Steel tape & 11.48 & 0.01 & 11.61 & 418.89 & MM \\
\hline $07-17-93$ & 1704 & Steel tape & 11.53 & 0.01 & 11.66 & 418.84 & MM \\
\hline $08-16-93$ & 1053 & Steel tape & 10.98 & 0.01 & 11.11 & 419.39 & MM \\
\hline $09-15-93$ & 1621 & Steel tape & 11.33 & 0.01 & 11.46 & 419.04 & $M M$ \\
\hline $10-19-93$ & 1336 & Steel tape & 11.88 & 0.01 & 12.01 & 418.49 & MM \\
\hline $10-28-93$ & 1349 & E-tape & 12.39 & 0.02 & 12.52 & 417.98 & PM \\
\hline $11-16-93$ & 1355 & Steel tape & 12.64 & 0.04 & 12.77 & 417.73 & MM \\
\hline $01-19-94$ & 1128 & Steel tape & 13.19 & 0.01 & 13.32 & 417.18 & $M M$ \\
\hline $02-03-94$ & 1504 & Steel tape & $\uparrow 3.46$ & 0.07 & 13.59 & 416.91 & PM \\
\hline $02-24-94$ & 1440 & Steel tape & 13.45 & 0.01 & 13.58 & 416.92 & $M M$ \\
\hline $03-09-94$ & 1542 & Steel tapa & 13.41 & 0.01 & 13.54 & 416.96 & PM \\
\hline $03-16-94$ & 1337 & Steet tape & 13.46 & 0.01 & 13.59 & 416.91 & $M M$ \\
\hline
\end{tabular}


G6 GROUND-WATER OBSERVATION WELL_ (ContinLed)

Site iD:

645054147494301

Local Number: $\quad$ FC00100107BAAB1

\begin{tabular}{cccccccc}
\hline Date & Time & Method & MP to WS & Error & LS to WS & WS elevation & Remarks \\
\hline $04-21-94$ & 1303 & Steel tape & 13.75 & 0.01 & 13.88 & 416.62 & MM \\
$06-03-94$ & 0916 & Steel tape & 13.71 & 0.01 & 13.84 & 416.66 & PM \\
$06-09-94$ & 1730 & Steel tape & 13.42 & 0.01 & 13.55 & 416.95 & PM \\
$06-15-94$ & 0916 & Steel tape & 13.34 & 0.01 & 13.47 & 417.03 & MM \\
$06-21-94$ & 1652 & Steel tape & 12.35 & 0.01 & 12.48 & 418.02 & PM \\
$07-01-94$ & 1534 & Steel tape & NA & 0.01 & NA & NA & PM, unable to open lock \\
$07-15-94$ & 1015 & Steel tape & 11.04 & 0.01 & 11.17 & 419.33 & MM \\
$08-16-94$ & 0925 & Steel tape & 10.54 & 0.01 & 10.67 & 419.83 & MM \\
$09-15-94$ & 1142 & Steel tape & 12.18 & 0.01 & 12.31 & 418.19 & MM \\
$09-29-94$ & 1338 & Steel tape & 13.06 & 0.01 & 13.19 & 417.31 & PM \\
$10-20-94$ & 1247 & Steel tape & 13.75 & 0.01 & 13.88 & 416.62 & MM \\
\hline
\end{tabular}


GROUND-WATER ELEVATION, IN FEET ABOVE SEA LEVEL

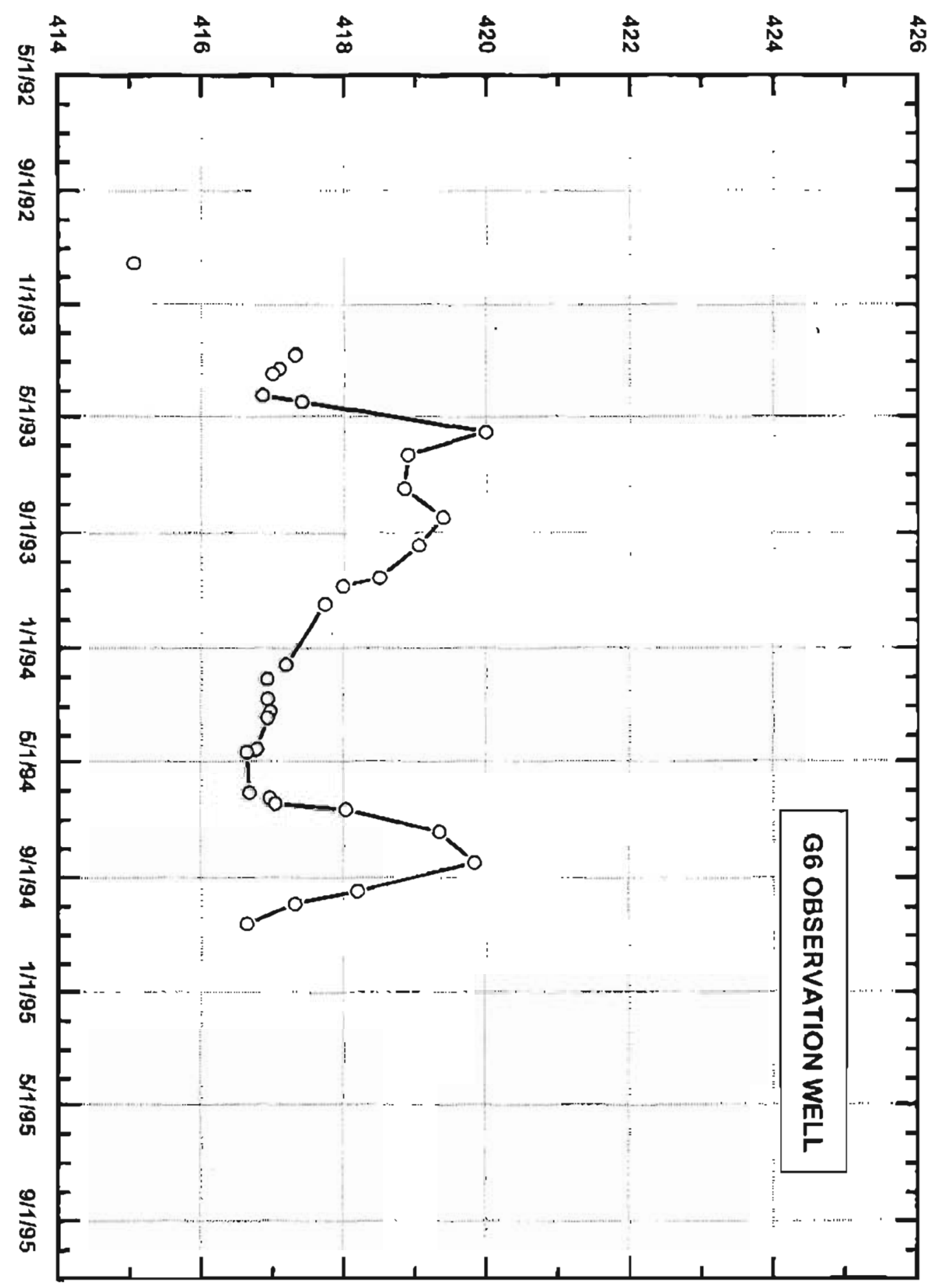


GW1B WATER-SUPPLY WELL

\section{CONTINUOUS-DATA COLLECTION STATION}

North Well, Outside of Fence

All measurements in feet

Depth to bottom of well from MP :

Depth to Pump Inlet from MP :

Land Surfaçe Dałum:

\begin{tabular}{c|c} 
Feel & Elevation \\
\hline 70.0 & 367.3 \\
42.0 & 395.3 \\
& 435.0
\end{tabular}

Datum corrections, reference survey notes in site folders

\begin{tabular}{c|c} 
Date & MP elevation (feet above sea level) \\
\hline $08-04-92$ & 437.28 \\
$08-17-93$ & 437.18
\end{tabular}

Site ID:

Local Number: 645104147494002 FC00100106CDDB1

LS, land surface $M M$, mass measurement $M P$, measuring point NA, not available $P M_{2}$, partial measurement PT, pump tes!

SI, screened interval TOC, top of casing WS, water surface

\begin{tabular}{|c|c|c|c|c|c|c|c|c|}
\hline Date & Time & Method & MP to WS & ErTor & LS to WS & WS elevation & $\begin{array}{l}\text { Flow Rate } \\
\text { (gal/min) }\end{array}$ & Remarks \\
\hline $06-17-92$ & 1730 & Steel tape & 18.47 & 0.01 & 16.29 & 418.81 & 0 & PM \\
\hline $07-17-92$ & 1400 & Steel tape & 20.02 & 0.01 & 17.84 & 417.26 & 0 & MM \\
\hline $08-13-92$ & 2015 & Steel tape & 20.36 & 0.01 & 18.18 & 416.92 & 0 & $\mathrm{MM}$ \\
\hline $08-14-92$ & 1530 & Steel tape & 20.38 & 0.01 & $\{8.20$ & 416.90 & 0 & $P M$ \\
\hline $09-14-92$ & 1827 & Steel tape & 22.80 & 0.01 & 20.42 & 414.88 & 0 & $P M$ \\
\hline $11-06-92$ & 1335 & Steel tape & 24.11 & 0.01 & 21.93 & 413.17 & 0 & MM \\
\hline $12-14-92$ & 1512 & Steel tape & 22.90 & 0.01 & 20.72 & 414.38 & 0 & MM \\
\hline $01-30-93$ & 1235 & Steel tape & 21.98 & 0.01 & 19.80 & 415.30 & 0 & $\mathrm{PM}$ \\
\hline $01-31-93$ & 1841 & Steel tape & 21.99 & 0.01 & 19.81 & 415.29 & 0 & PM, installed instr sheiter \\
\hline $02-10-93$ & 1640 & Steel tape & 22.24 & 0.01 & 20.06 & $4 \div 5.04$ & 0 & $P A$ \\
\hline $02-13-93$ & 1548 & Steel tape & 22.19 & 0.01 & 20.01 & 415.09 & 275 & PM \\
\hline $02-15-93$ & 1350 & Steel tape & 22.10 & 0.01 & 19.92 & 415.18 & 305 & PT \\
\hline $02-15-93$ & 1518 & Steel tape & 22.05 & 0.01 & 19.87 & 415.23 & 0 & PT, Pump off 20 minutes \\
\hline $02-15-93$ & 1535 & Steel tape & 23.25 & 0.01 & 21.07 & 414.03 & 455 & $F T$ \\
\hline $02-15-93$ & 1608 & Steel tape & 22.12 & 0.01 & 19.94 & 415.16 & 280 & PT \\
\hline $02-19-93$ & 0947 & Steel tape & 22.09 & 0.01 & 19.91 & 415.19 & 0 & $M M$ \\
\hline $02-22-93$ & 1550 & Steel tape & 20.82 & 0.01 & 18.64 & 416.46 & 0 & MM \\
\hline $02-24-93$ & 1033 & Steel tape & 23.11 & 0.01 & 20.93 & 414.17 & 435 & $\mathrm{PM}$ \\
\hline
\end{tabular}


GW1B WATER-SUPPLY WELL

(Continued)

Site ID:

645104147494002

CONTINUOUS-DATA COLLECTION STATION

Local Number:

FC00100106CDDB1

\begin{tabular}{|c|c|c|c|c|c|c|c|c|}
\hline Date & Time & Method & MP to WS & Error & LS to WS & WS elevation & $\begin{array}{c}\text { Flow Rate } \\
\text { (gal/min) }\end{array}$ & Remarks \\
\hline $02-25-93$ & 1145 & Steel tape & 20.68 & 0.01 & 18.50 & 416.60 & 0 & $M M$ \\
\hline $03-12-93$ & 1138 & Steel tape & 21.72 & 0.01 & 19.54 & 415.56 & 190 & PM, 130 psi \\
\hline $04-09-93$ & 1240 & Steel tape & 22.39 & 0.01 & 20.21 & 414.89 & 0 & $P M$ \\
\hline $04-16-93$ & 0855 & Steel tape & 21.79 & 0.01 & 19.61 & 415.49 & 250 & MM, 120 psi \\
\hline $05-18-93$ & 1512 & Steel tape & 19.11 & 0.01 & 16.93 & 418.17 & 0 & $M M$ \\
\hline $06-11-93$ & 1035 & Steel tape & 19.55 & 0.01 & 17.37 & 417.73 & 190 & $M M_{1} 128$ psi \\
\hline $07-06-93$ & 1525 & Steel tape & 19.98 & 0.01 & 17.80 & 417.30 & 220 & PM, 125 psì \\
\hline $07-16-93$ & 1107 & Steel tape & 21.47 & 0.01 & 19.29 & 415.91 & 400 & MM, 85 psi \\
\hline $08-16-93$ & 1025 & Steel tape & 19.62 & 0.01 & 17.44 & 417.66 & 200 & MM, $123 \mathrm{psi}$ \\
\hline $09-16-93$ & 1352 & $\varepsilon$-tape & 20.55 & 0.02 & 18.37 & $4 \uparrow 6.63$ & 290 & MM, 105 psi \\
\hline $10-19-93$ & 1315 & Steel tape & 21.85 & 0.01 & 19.67 & 415.33 & 290 & MM, $100 \mathrm{ps}$ \\
\hline $10-27-93$ & 1646 & Steel tape & NA & 0.01 & NA & NA & 0 & PM, well lock frozen, 0 osi \\
\hline $10-27-93$ & 1744 & E-tape & 19.76 & 0.02 & 17.58 & 417.42 & 0 & $P M, O p s i$ \\
\hline $10-28-93$ & 1330 & E-tape & 19.68 & 0.02 & 17.50 & 417.50 & 0 & PM, 0 psi \\
\hline $11-16-93$ & 1624 & Steel tape & 21.27 & 0.01 & 19.09 & 415.91 & 280 & MAM, 100 psi \\
\hline $12-22-93$ & 1547 & E-tape & 20.72 & 0.02 & 18.54 & 416.46 & 150 & MM, 120 psi \\
\hline $01-05-94$ & 1040 & E-tape & 20.78 & 0.02 & 18.60 & 416.40 & 160 & $P M, 120$ psi \\
\hline $01-19-94$ & 1050 & Steel tape & 22.08 & 0.01 & 19.90 & 415.10 & 270 & MM, 95 psi \\
\hline $03-16-94$ & 1541 & Steel tape & 22.38 & 0.01 & 20.20 & 414.80 & 250 & MN, 95 psi \\
\hline $04-21-94$ & 1406 & Steel tape & 22.29 & 0.01 & 20.11 & 414.89 & 220 & MM, 95 psi \\
\hline $05-16-94$ & 2102 & Steel tape & 20.61 & 0.01 & 18.43 & 416.57 & 150 & MM, $110 \mathrm{psi}$ \\
\hline $06-15-94$ & 1010 & Steel tape & 22.34 & 0.01 & 20.16 & 414.84 & 0 & $M M$ \\
\hline $07-15-94$ & 0910 & Steel tape & 19.62 & 0.01 & 17.44 & 417.56 & 0 & MM, 0 psi \\
\hline$O B-15-94$ & 0938 & Steel tape & 19.01 & 0.01 & 16.83 & 418.17 & 0 & MM, 100 psi \\
\hline $09-15-94$ & 1318 & Steel tape & 20.58 & 0.01 & 18.40 & 416.60 & 0 & MM, 75 psi \\
\hline $10-20-94$ & 1420 & Steel tape & 22.22 & 0.01 & 20.04 & 414.96 & 0 & $\mathrm{MH}, 70 \mathrm{psi}$ \\
\hline $10-26-94$ & 1356 & Steel tape & 22.26 & 0.01 & 20.08 & 414.92 & 0 & PM, 75 psi \\
\hline $11-16-94$ & 1510 & Steel tape & 22.22 & 0.01 & 20.04 & $4\} 4.96$ & 220 & MM, 75 psi \\
\hline $12-23-94$ & 1635 & Steel tape & NA & $\mathrm{NA}$ & $N A$ & NA & 150 & MM, 87 psi \\
\hline $02-16-95$ & 1655 & Steel tape & 22.45 & 0.01 & 20.27 & 414.73 & 200 & MM, $72 \mathrm{ps}$ \\
\hline
\end{tabular}




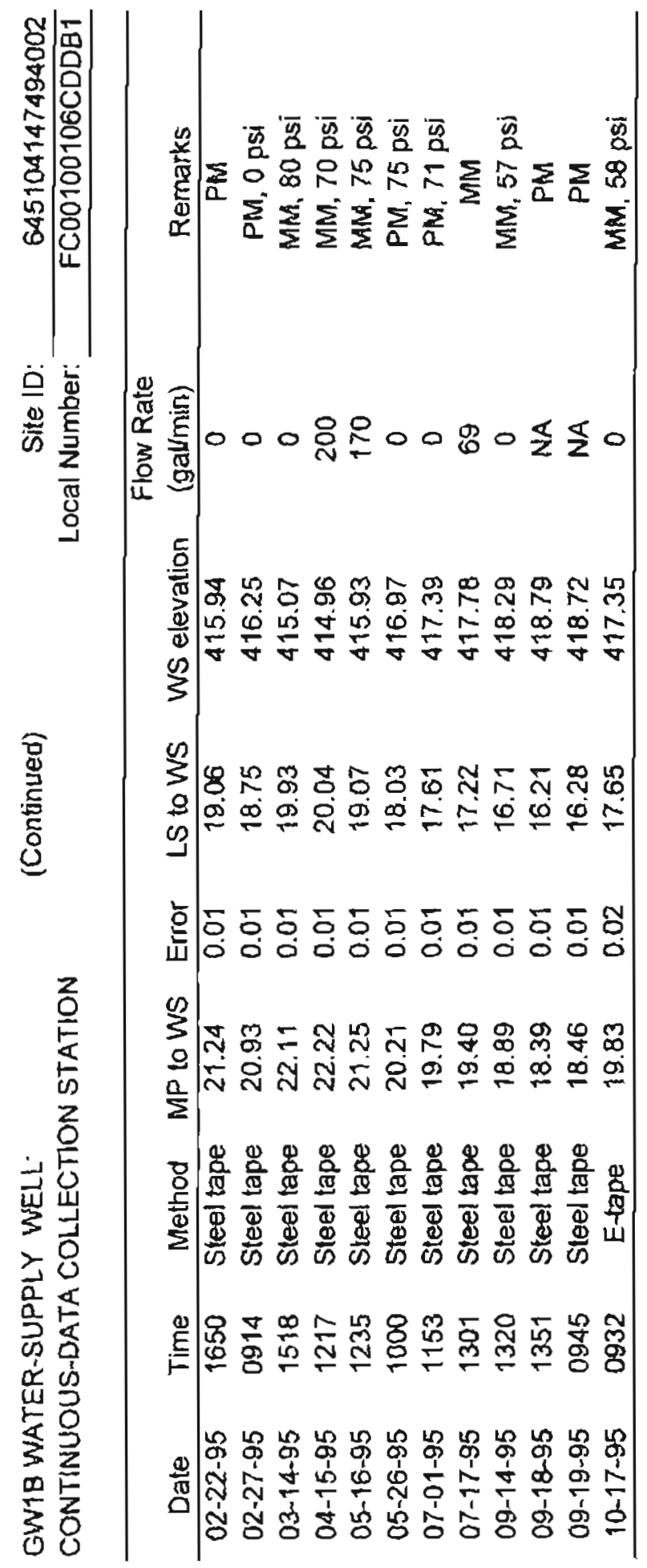




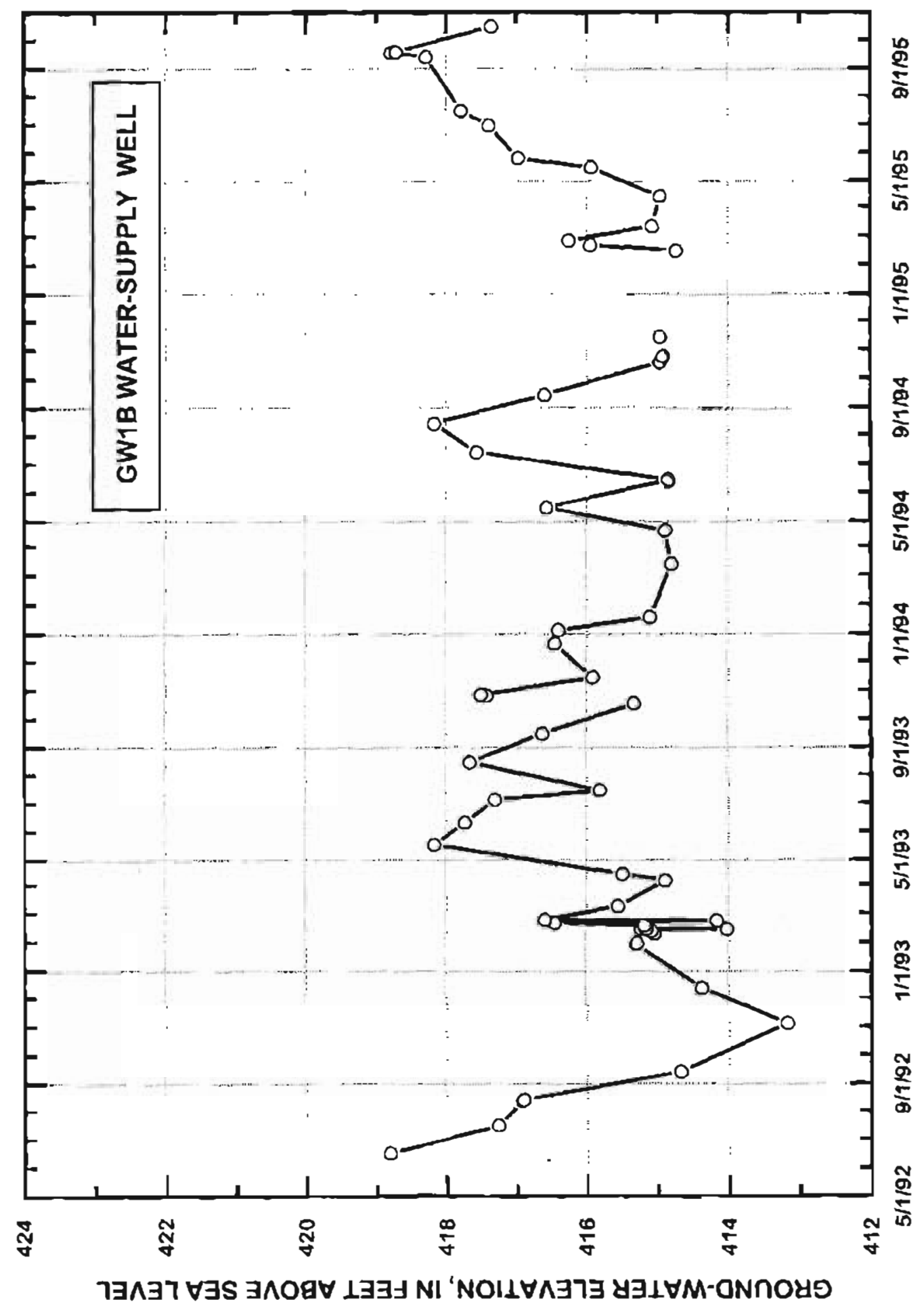


GW1C ABANDONED WATER-SUPPLY WELL CONTINUOUS-DATA COLLECTION STATION

North Wel!, Inside Well House

All measurements in feet

Depth to bottom of well from MP :

Depth to Pump Inlet from MP :

Land Surface Datum:

\begin{tabular}{c|c} 
Feet & Elevation \\
\hline$>30$ & NA \\
NA & NA \\
& 436.0
\end{tabular}

Datum corrections, reference survey notes in site folders

\begin{tabular}{c|c} 
Date & MP elevation (feet above sea level) \\
\hline $08-04-92$ & $\overline{437.98}$
\end{tabular}
Site ID: Local Number:

LS, land surface

$M M$, mass measurement

$M P$, measuring point

$N A$, not available

$\mathrm{PM}_{\text {, partial measurement }}$

PT, pump test

SI, screened interval

TOC, top of casing

WS, water surface

\begin{tabular}{cccccccc}
\hline Date & Time & Method & MP to WS & Error & LS to WS & WS elevation & Remarks \\
\hline $07-17-92$ & 1430 & Steel tape & 20.78 & 0.01 & 18.80 & 417.20 & MM \\
$08-13-92$ & 2013 & Steel tape & 21.03 & 0.01 & 19.05 & 416.95 & MM \\
$09-14-92$ & 1840 & Steel tape & 23.25 & 0.01 & 21.27 & 414.73 & PM \\
$11-06-92$ & 1330 & Steel tape & 24.03 & 0.01 & 22.05 & 413.95 & MM \\
$12-14-92$ & 1409 & Steel tape & 22.79 & 0.01 & 20.81 & 415.20 & MM \\
$01-30-93$ & 0944 & Steel tape & 21.77 & 0.01 & 19.79 & 416.21 & PM, installed instr. \\
$02-10-93$ & 1520 & Steel tape & 21.95 & 0.01 & 19.97 & 416.03 & PM \\
$02-13-93$ & 1620 & Steel tape & 22.02 & 0.01 & 20.04 & 415.86 & PM \\
$02-15-93$ & 1345 & Steel tape & 21.81 & 0.01 & 19.83 & 416.17 & MM, PT \\
$02-15-93$ & 1515 & Steel tape & 21.81 & 0.01 & 19.83 & 416.17 & MM, PT \\
$02-19-93$ & 0940 & Steel tape & 21.97 & 0.01 & 19.99 & 416.01 & MM \\
$02-22-93$ & 1543 & Steel tape & 21.59 & 0.01 & 19.61 & 416.39 & MM \\
$02-24-93$ & 0858 & Steel tape & 21.34 & 0.01 & 19.36 & 416.64 & PM \\
$02-24-93$ & 1054 & Steel tape & 21.70 & 0.01 & 19.72 & 416.28 & PM \\
$02-25-93$ & 1150 & Steel tape & 21.39 & 0.01 & 19.41 & 416.59 & MM \\
$02-28-93$ & 1549 & Steel tape & 21.88 & 0.01 & 19.90 & 416.10 & PM \\
$03-01-93$ & 1645 & Steel tape & 22.11 & 0.01 & 20.13 & 415.87 & PMA \\
$03-12-93$ & 1156 & Steel tape & 21.81 & 0.01 & 19.83 & 416.17 & MM \\
$04-16-93$ & 0850 & Steel tape & 21.66 & 0.01 & 19.68 & 416.32 & MM \\
$06-11-93$ & 1027 & Steel tape & 19.60 & 0.01 & 17.62 & 418.38 & MM
\end{tabular}


GW1C ABANDONED WATER-SUPPLY WELL CONTINUOUS-DATA COLLECTION STATION
(Continued)
Site lD:

645104147494004

Local Number: FC00100106CDDB2

\begin{tabular}{|c|c|c|c|c|c|c|c|}
\hline Date & Time & Method & MP to WS & Error & LS to WS & WS elevation & Remarks \\
\hline $07-06-93$ & 1515 & Steel tape & 19.93 & 0.01 & 17.95 & $4 \nmid 8.05$ & $M A$ \\
\hline $07-16-93$ & 1112 & Steel tape & 20.58 & 0.01 & 18.60 & 417.40 & MM \\
\hline $08-16-93$ & 1030 & Steel tape & 19.63 & 0.01 & 17.65 & 418.35 & MM \\
\hline $09-16-93$ & 1347 & E-tape & 20.16 & 0.02 & 18.18 & 417.82 & MM \\
\hline $10-19-93$ & 1309 & Steel tape & 20.43 & 0.01 & $\$ 8.45$ & 417.55 & MM \\
\hline $10-27-93$ & 1644 & E-tape & 20.69 & 0.02 & 18.71 & 417.29 & $\mathrm{PM}$ \\
\hline $10-28-93$ & 1327 & E-tape & 20.39 & 0.02 & 18.41 & 417.58 & $P M$ \\
\hline $11-16-93$ & 1615 & Steel tape & 21.63 & 0.01 & 19.65 & 416.35 & MM \\
\hline $12-22-93$ & 1500 & E-tape & 20.94 & 0.02 & 18.96 & 417.04 & MM \\
\hline $01-04-94$ & 1646 & E-tape & 20.90 & 0.02 & B. 92 & $4\{7.08$ & $\mathrm{PM}$ \\
\hline $01-19-94$ & 1059 & Steel tape & 21.72 & 0.01 & 19.74 & 416.26 & MM \\
\hline $02-23-94$ & 1218 & Steel tape & 22.05 & 0.01 & 20.07 & 415.93 & MM \\
\hline $03-16-94$ & 1547 & Steel tape & 22.12 & 0.01 & 20.14 & 415.86 & MM \\
\hline $04-21-94$ & 1402 & Steel tape & 22.21 & 0.01 & 20.23 & 415.77 & $M M$ \\
\hline $05-16-94$ & 2047 & Steel tape & 20.84 & 0.01 & 18.86 & 417.14 & MM \\
\hline $06-15-94$ & 1017 & Steel tape & 21.91 & 0.03 & 19.93 & 416.07 & $M M$ \\
\hline $07-15-94$ & 0902 & Steel tape & 18.51 & 0.01 & 16.53 & 419.47 & MM \\
\hline $07-20-94$ & 1844 & Steel tape & 19.51 & 0.01 & 17.53 & 418.47 & PM \\
\hline $08-15-94$ & 0935 & Steel tape & 19.21 & 0.01 & 17.23 & 418.77 & $\mathrm{MM}$ \\
\hline $09-15-94$ & 1317 & Steel tape & 20.38 & 0.01 & 18.40 & 417.60 & MM \\
\hline $10-20-94$ & 1422 & Steel tape & 21.99 & 0.01 & 20.01 & 415.99 & MM \\
\hline $10-26-94$ & 1348 & Steel tape & 22.12 & 0.01 & 20.14 & 415.86 & $P M$ \\
\hline $11-16-94$ & 1500 & Steel tape & 22.18 & 0.01 & 20.20 & 415.80 & MM \\
\hline $12-23-94$ & 1531 & Steel tape & 21.74 & 0.01 & 19.76 & 416.24 & MM \\
\hline $02-16-95$ & 1652 & Steel tape & 22.51 & 0.01 & 20.53 & $4 \nmid 5.47$ & MM \\
\hline $02-22-95$ & 1710 & Steel tape & 22.06 & 0.01 & 20.08 & 415.92 & $P M$ \\
\hline $02-27-95$ & 0915 & Steel tape & 21.65 & 0.01 & 19.67 & 416.33 & PM \\
\hline $03-14-95$ & 1525 & Steel tape & 22.38 & 0.01 & 20.40 & 415.60 & MM \\
\hline $04-15-95$ & 1212 & Steel tape & 22.33 & 0.01 & 20.35 & 415.65 & MM \\
\hline $05-16-95$ & 1227 & Steel tape & 20.56 & 0.01 & 18.58 & 417.42 & MM \\
\hline $05-26-95$ & 0937 & Steel tape & 20.49 & 0.01 & 18.51 & 417.49 & PM \\
\hline
\end{tabular}


GW1C ABANDONED WATER-SUPPLY WELL CONTINUOUS-DATA COLLECTION STATION

(Continued)

Site ID

Local Number:

645104147494004

\begin{tabular}{cccccccc}
\hline Date & Time & Method & MP to WS & Error & LS to WS & WS elevation & Remarks \\
\hline $07-01-95$ & 1142 & Steed tape & 20.07 & 0.01 & 18.09 & 417.91 & PM \\
$07-17-95$ & 1306 & Steel tape & 19.18 & 0.01 & 17.20 & 418.80 & MM \\
$09-14-95$ & 1313 & Steel tape & 18.90 & 0.01 & 16.82 & 419.08 & MM \\
$09-18-95$ & 1545 & Steel tape & 18.66 & 0.01 & 16.68 & 419.32 & PM \\
$10-17-95$ & 0928 & E-tape & 19.88 & 0.02 & 17.90 & 418.10 & MM \\
\hline
\end{tabular}




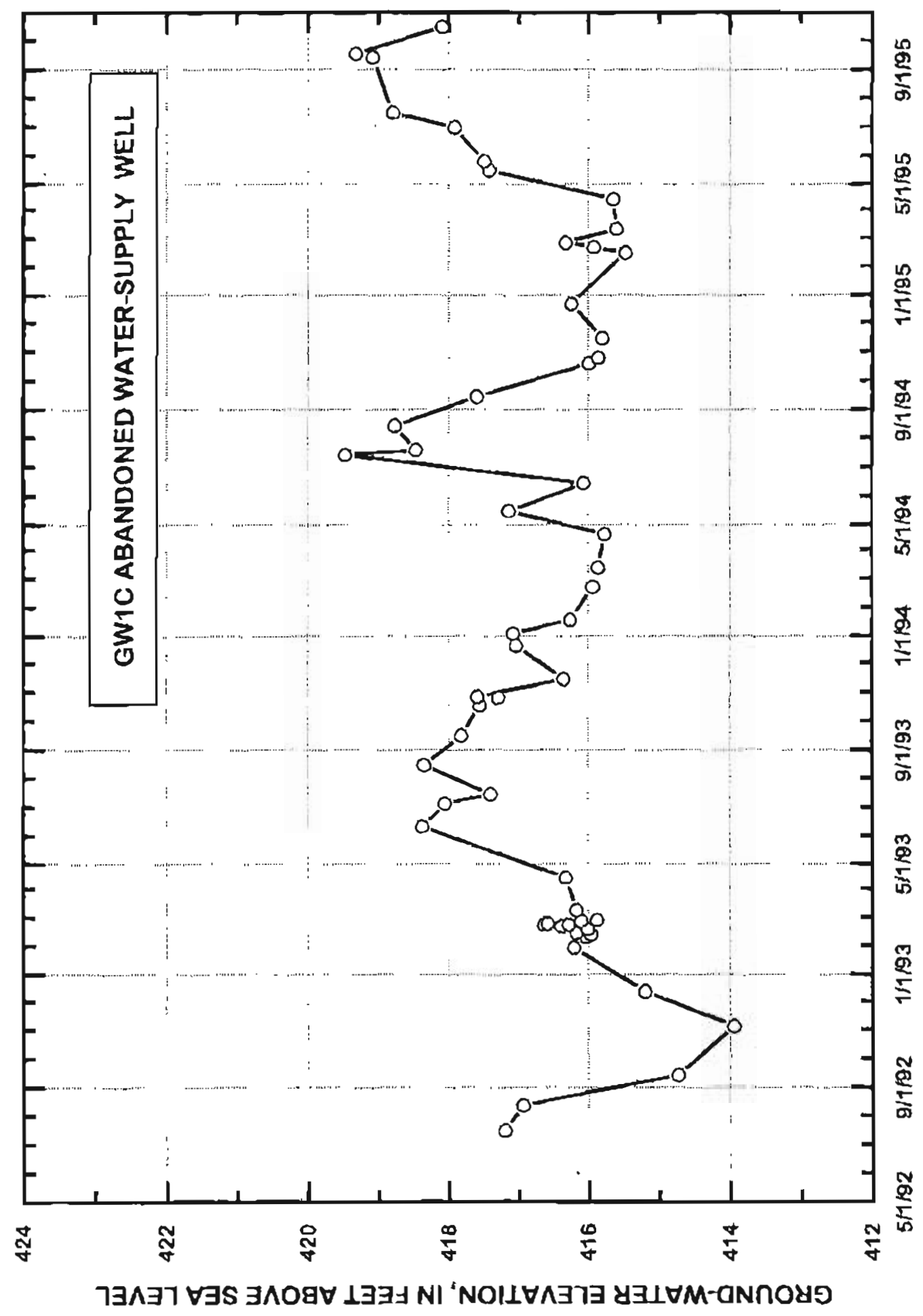



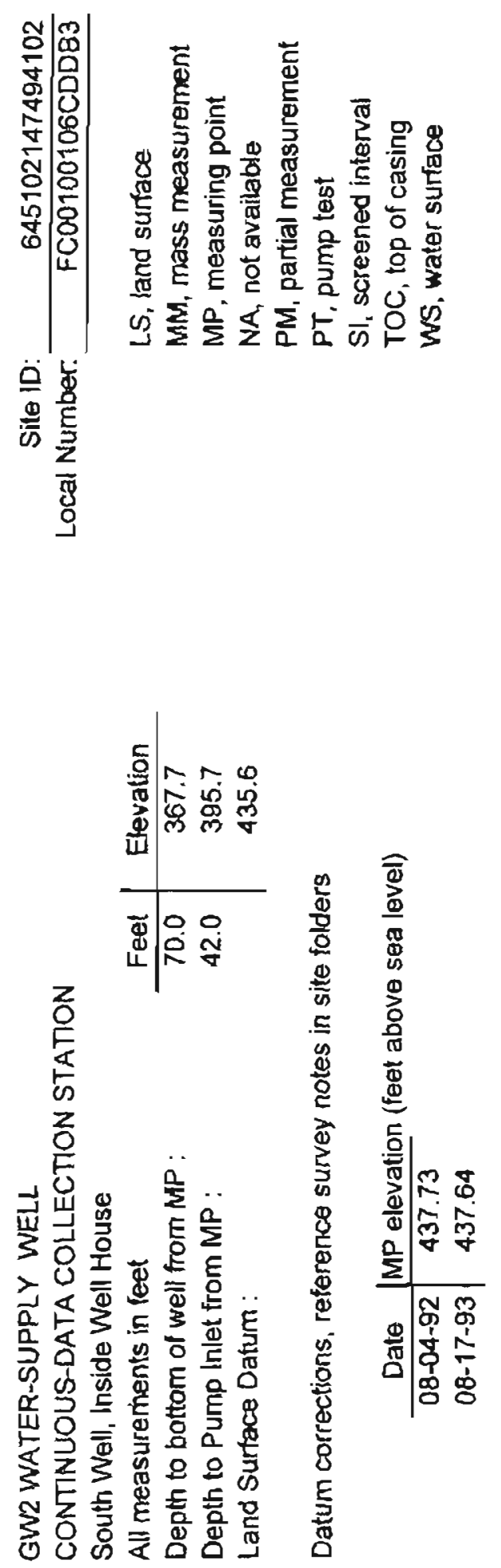

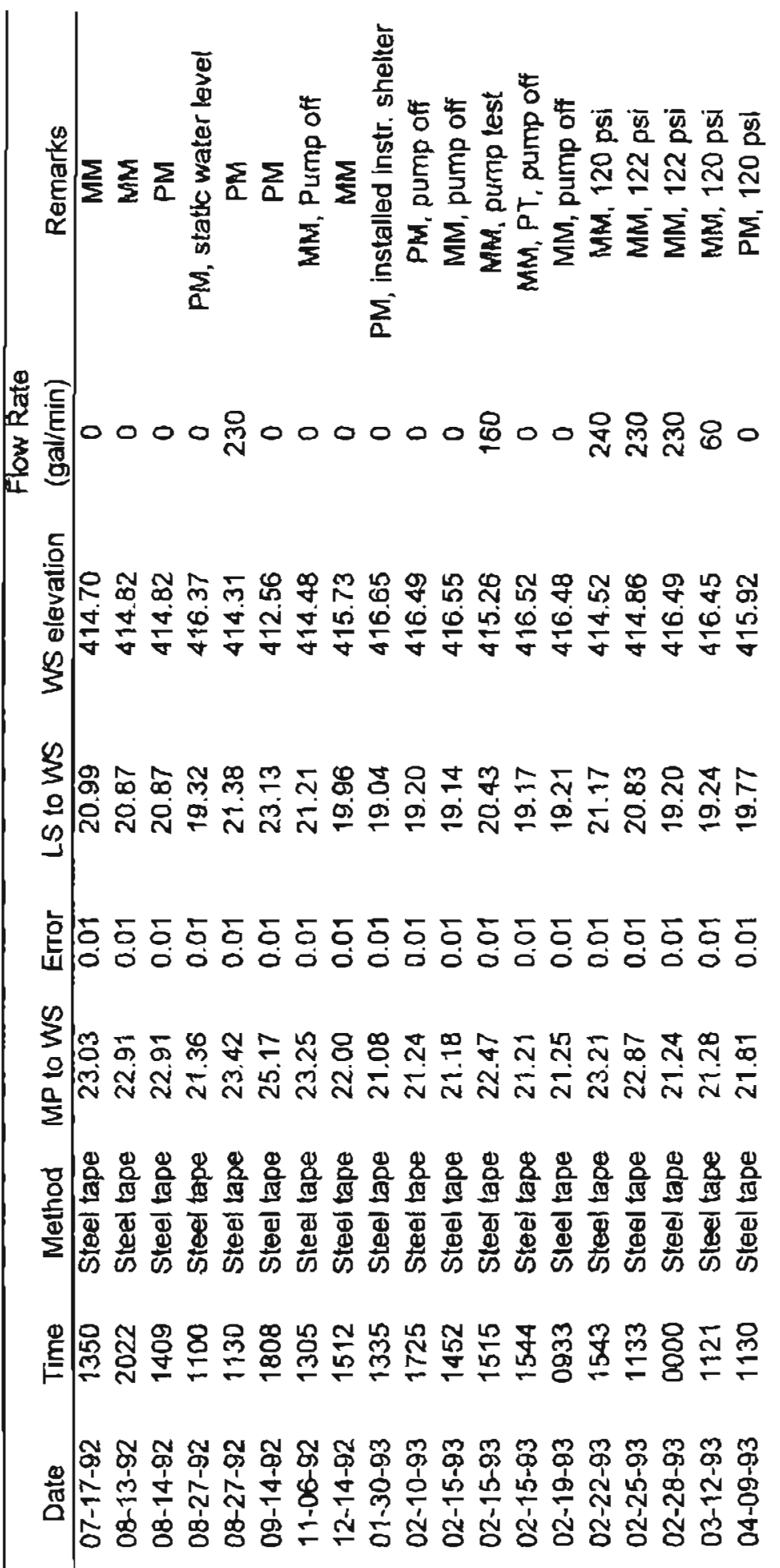


GW2 WATER-SUPPLY WELL CONTINUOUS-DATA COLLECTION STATION

Site ID:

645102147494102

Local Number: FC00100106CDDB3

\begin{tabular}{|c|c|c|c|c|c|c|c|c|}
\hline Date & Time & Wethod & MP to WS & Error & LS to WS & WS elevation & $\begin{array}{c}\text { Flow Rate } \\
\text { (gal/min) }\end{array}$ & Remarks \\
\hline $04-16-93$ & 0900 & Steel tape & 21.08 & 0.01 & 19.04 & 416.65 & 0 & MM, 120 psi \\
\hline $06-11-93$ & 1042 & Steel tape & 19.14 & 0.01 & 17.10 & 418.50 & 0 & MM \\
\hline $07-06-93$ & 1600 & Steel tape & 19.35 & 0.01 & 17.31 & 418.29 & 0 & $\mathrm{PM}_{4} \uparrow 18 \rho \mathrm{pi}$ \\
\hline $07-16-93$ & 1117 & Steel tape & 23.03 & 0.01 & 20.99 & 414.61 & 300 & MM, 127 psi \\
\hline $08-16-93$ & 1034 & Steel tape & 19.06 & 0.01 & 17.02 & 418.58 & 0 & MM, 121 psi \\
\hline $09-16-93$ & 1358 & E-tape & 19.49 & 0.02 & 17.45 & 418.15 & 0 & MM, 122 psi \\
\hline $10-19-93$ & 1325 & Steel tape & 19.81 & 0.01 & 17.77 & 417.83 & 0 & $\mathrm{MM}$ \\
\hline $10-27-93$ & 1702 & E-tape & 22.67 & 0.02 & 20.63 & 414.97 & 290 & PM, 100 psi \\
\hline $10-28-93$ & 1340 & E-tape & 22.36 & 0.02 & 20.32 & 415.28 & 290 & PM, 100 psi \\
\hline $11-16-93$ & 1633 & Steel tape & 20.64 & 0.01 & 18.60 & 417.00 & 0 & PM, 100 psi \\
\hline $01-04-94$ & 1635 & E-tape & 20.47 & 0.02 & 18.43 & 417.17 & 0 & PM, 128 psi \\
\hline $01-19-94$ & 1031 & Steel tape & 21.12 & 0.01 & 19.08 & 416.52 & 0 & MM, $128 \mathrm{psi}$ \\
\hline $02-23-94$ & 1210 & Steel tape & 21.46 & 0.01 & 19.42 & 416.18 & 0 & MM, 100 psi \\
\hline $03-\uparrow 6-94$ & 1551 & Steel tape & 21.50 & 0.01 & 19.46 & 416.14 & 0 & MM, $100 \mathrm{psi}$ \\
\hline $04-21-94$ & 1328 & Steel tape & 21.61 & 0.01 & 19.57 & 416.03 & 0 & MM, 100 psi \\
\hline $05-16-94$ & 2031 & Steel tape & 20.35 & 0.01 & 18.31 & 417.29 & 0 & MM, $100 \mathrm{psi}$ \\
\hline $06-15-94$ & 0950 & Steel tape & 21.31 & 0.01 & 19.27 & 416.33 & 0 & MM \\
\hline $06-27-94$ & 1625 & Steel tape & 19.51 & 0.01 & 17.47 & 418.13 & 0 & PM \\
\hline $07-15-94$ & 0918 & Steel tape & 18.86 & 0.01 & 16.82 & 418.78 & 0 & $\mathrm{MM}_{1} 97 \mathrm{psi}$ \\
\hline $07-21-94$ & 1020 & Steel tape & 18.98 & 0.01 & 16.94 & 418.66 & 0 & PM, 98 psi \\
\hline $08-15-94$ & 0958 & Steel tape & 19.60 & 0.01 & 17.56 & 418.04 & 0 & MM, 96 psi \\
\hline $09-15-94$ & 1332 & Steel tape & 19.83 & 0.01 & 17.79 & 417.81 & 0 & MM, 78 psi \\
\hline $10-20-94$ & 1428 & Steel tape & 21.42 & 0.01 & 19.38 & 416.22 & 0 & MM, 70 psì \\
\hline $10-26-94$ & 1411 & Steel tape & 21.54 & 0.01 & 19.50 & 416.10 & 0 & PM \\
\hline $11-16-94$ & 1450 & Steel tape & 21.64 & 0.01 & 19.60 & 416.00 & 0 & MM, 78 psi \\
\hline $12-23-94$ & 1524 & Steel tape & 21.25 & 0.01 & 19.21 & 416.39 & 0 & MM, 85 psi \\
\hline $02-16-95$ & 1705 & Steel tape & 21.16 & 0.01 & 19.12 & 416.48 & 250 & MM, 80 psi \\
\hline $02-22-95$ & 1640 & Steel tape & 21.68 & 0.01 & 19.64 & 415.96 & 0 & PM \\
\hline $02-27-95$ & 0852 & Steel tape & 21,36 & 0.01 & 19.32 & 416.28 & 0 & PM, 126 psi \\
\hline 03-14-95 & 1533 & Steel tape & 21.88 & 0.01 & 19.84 & 415.76 & 0 & MM, 80 psi \\
\hline
\end{tabular}




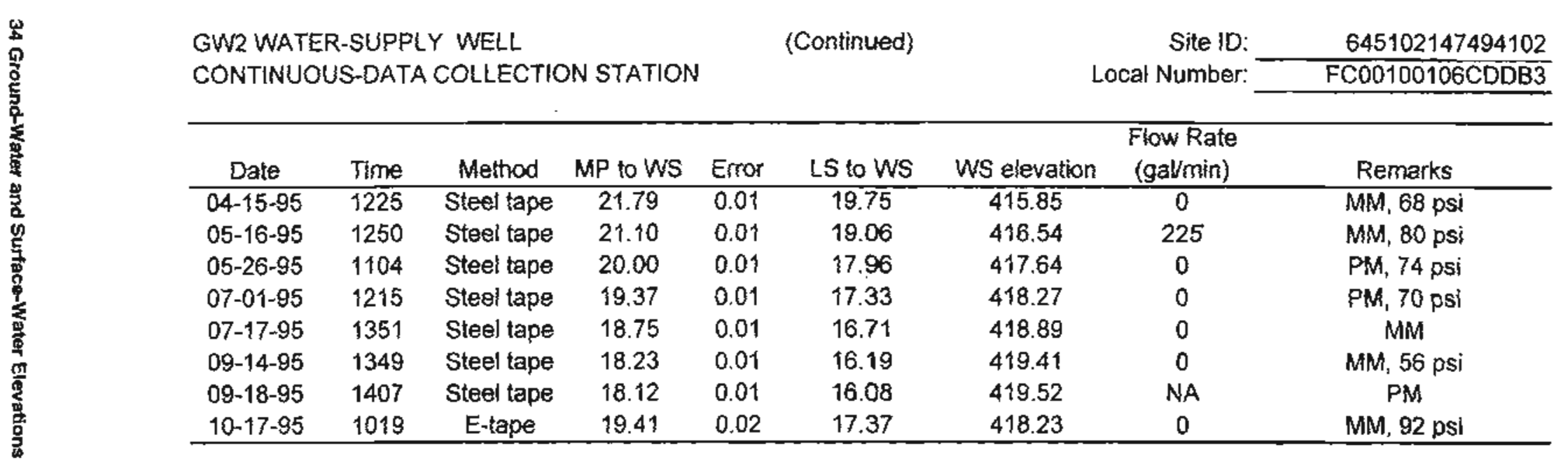




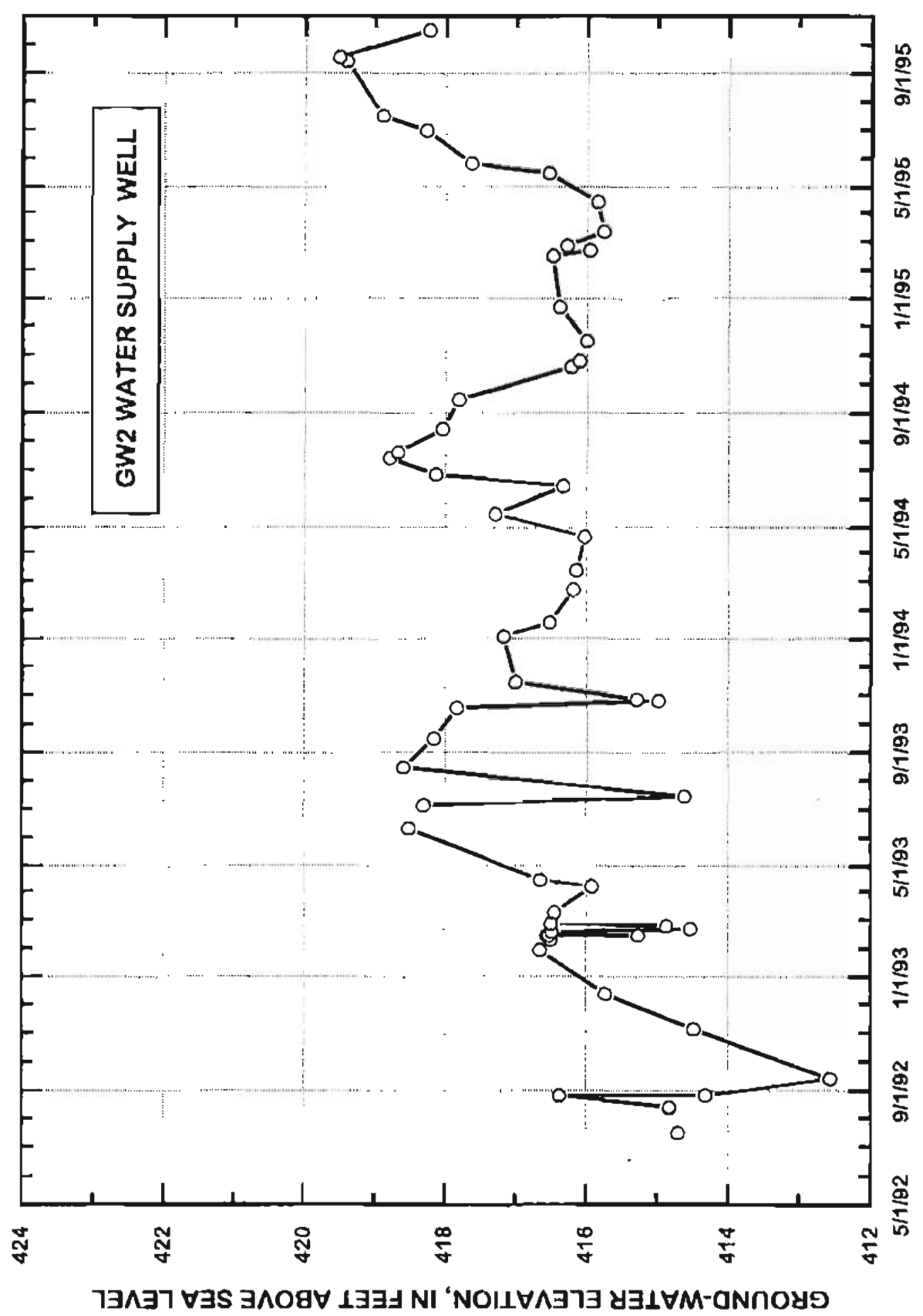




\section{MW101 GROUND-WATER OBSERVATION WELL}

Near Power Plant

All measurements in feet

Depth to bottom of well from MP :

Depth from TOC to top of SI :

Depth from TOC to bottom of SI :

Land Surface Datum :

\begin{tabular}{c|c} 
Feet & Elevation \\
\hline 29.5 & 408.9 \\
6.5 & 431.9 \\
25.6 & 412.8 \\
& 435.1
\end{tabular}

Datum corrections, reference survey notes in site folders

$$
\begin{array}{c|c}
\text { Date } & \text { MP Elevation (feet above sea level) } \\
\hline 07-01-92 & 436.44
\end{array}
$$

Site ID:

Local Number:

645112147491601 FC00100106DBCA1

LS, land surface

$M M$, mass measurement

MP, measuring point

$N A$, not available

$\mathrm{PM}_{\mathrm{r}}$ partial measurement

SI, screened interval

TOC, top of casing

WS, water surface

\begin{tabular}{|c|c|c|c|c|c|c|c|}
\hline Date & Time & Method & MP to WS & Error & LS to WS & WS elevation & Remarks \\
\hline $06-18-92$ & 1704 & Steel tape & 19.21 & 0.01 & 15.87 & 419.23 & $\mathrm{PM}$ \\
\hline $07-17-92$ & 1455 & Steel tape & 21.11 & 0.01 & 17.77 & 417.33 & MM \\
\hline $08-13-92$ & 2001 & Steel tape & 22.01 & 0.01 & 18.67 & 416.43 & MM \\
\hline $09-14-92$ & 1857 & Steel tape & 24.48 & 0.01 & 21.14 & 413.96 & PM \\
\hline 11-06-92 & 1245 & Steel tape & 23.48 & 0.01 & 20.14 & 414.96 & MA \\
\hline $12-03-92$ & 1000 & Steet tape & 22.48 & 0.01 & 19.14 & 415.96 & MM \\
\hline $12-14-92$ & 1040 & Steel tape & 22.12 & 0.01 & 18.78 & 416.32 & MM \\
\hline $02-24-93$ & 1632 & Steel tape & 21.01 & 0.01 & 17.67 & 417.43 & PM \\
\hline $02-25-93$ & 1323 & Steel tape & 20.95 & 0.01 & 17.61 & 417.49 & MM \\
\hline $03-12-93$ & 1410 & Steel tape & 21.20 & 0.01 & 17.86 & 417.24 & MM \\
\hline $03-17-93$ & 1518 & Steel tape & 21.32 & 0.01 & 17.98 & 417.12 & PM \\
\hline $04-02-93$ & 1705 & Steol tape & 21.48 & 0.01 & 18.14 & 416.96 & PM \\
\hline $04-09-93$ & 1404 & Steel tape & 21.21 & 0.01 & 17.87 & 417.23 & PM \\
\hline $04-12-93$ & 1652 & Sleel tape & 20.98 & 0.01 & 17.64 & 417.46 & PM \\
\hline $04-14-93$ & 1340 & Steet tape & 20.60 & 0.01 & 17.26 & 417.84 & PH \\
\hline $04-16-93$ & 1510 & Steel tape & 20.15 & 0.01 & 16.81 & 418.29 & MM \\
\hline $04-19-93$ & 1755 & Steel tape & 19.63 & 0.01 & 16.29 & 418.81 & PM \\
\hline $04-21-93$ & 1558 & Steel tape & 19.33 & 0.01 & 15.99 & 419.11 & PM \\
\hline $04-23-93$ & 1548 & Steel tape & 19.08 & 0.01 & 15.74 & 419.36 & PM \\
\hline $05-02-93$ & 1720 & Steel tape & 18.50 & 0.01 & 15.16 & 419.94 & $\mathrm{PM}$ \\
\hline
\end{tabular}


MW101 GROUND-WATER OBSERVATION WELL Near Power Plant
(Continued)

\begin{tabular}{|c|c|c|c|c|c|c|c|}
\hline Date & Time & Method & MP to WS & Error & LS to WS & WS elevation & Remarks \\
\hline $05-03-93$ & 1146 & Steed tape & 18.50 & 0.01 & 15.16 & 419.94 & PM \\
\hline $05-05-93$ & 1141 & Steel tape & 18.59 & 0.01 & 15.25 & 419.85 & $\mathrm{PM}$ \\
\hline $05-08-93$ & 2057 & Steel tape & 18.59 & 0.01 & 15.25 & 419.85 & PM \\
\hline $05-10-93$ & 1405 & Steel tape & 18.60 & 0.01 & 15.26 & $4 \uparrow 9.84$ & $\mathrm{PM}$ \\
\hline $05-12-93$ & 1020 & Steel tape & 18.58 & 0.01 & 15.24 & 419.86 & PM \\
\hline $05-14-93$ & 0959 & Steel tape & 18.49 & 0.01 & 15.15 & 419.95 & PM \\
\hline $05-18-93$ & 1017 & Steel tape & 18.42 & 0.01 & 15.08 & 420.02 & MAA \\
\hline $05-24-93$ & 1335 & Steel tape & 18.26 & 0.01 & 14.92 & 420.18 & $\mathrm{PM}$ \\
\hline $05-26-93$ & 1750 & Steel tape & 18.24 & 0.01 & 14.90 & 420.20 & PM \\
\hline $05-29-93$ & 1337 & Steel tape & 18.32 & 0.01 & 14.98 & 420.12 & $\mathrm{PM}$ \\
\hline $06-02-93$ & 1527 & Steel tape & 18.42 & 0.01 & 15.08 & 420.02 & PM \\
\hline $06-08-93$ & 1412 & Steel tape & 18.60 & 0.01 & 15.26 & 419.84 & PM \\
\hline $06-11-83$ & 1110 & Steel tape & 18.70 & 0.01 & 15.36 & 419.74 & $M M$ \\
\hline $06-2\}-93$ & 1105 & Steel tape & 18.98 & 0.01 & 15.64 & 419.46 & $P M$ \\
\hline $06-25-93$ & 1640 & Steel tape & 19.01 & 0.01 & 15.67 & 419.43 & PM \\
\hline $07-01-93$ & 1335 & E-tape & 19.01 & 0.02 & 15.67 & 419.43 & $P M$ \\
\hline $07-09-93$ & 0848 & Steel tape & 19.12 & 0.01 & $\{5.78$ & 419.32 & PM \\
\hline $07-16-93$ & 1209 & Steel tape & 19.23 & 0.01 & 15.89 & 419.21 & MM \\
\hline $07-21-93$ & 1428 & Steel tape & 19.24 & 0.01 & 15.90 & 419.20 & PM \\
\hline $07-28.93$ & 1731 & Steel tape & 19.14 & 0.01 & 15.80 & 419.30 & $P M$ \\
\hline $08-05-93$ & 1818 & Steel tape & 19.14 & 0.01 & 15.80 & 419.30 & $\mathrm{PM}$ \\
\hline $08-11-93$ & 1405 & Steel tape & 18.99 & 0.01 & 15.65 & 419.45 & $\mathrm{PM}$ \\
\hline $08-16-93$ & 0919 & Sleel tape & 18.97 & 0.01 & 15.63 & 419.47 & $M M$ \\
\hline $08-27-93$ & 1920 & Steel tape & $\uparrow 9.03$ & 0.01 & 15.69 & 419.41 & PM \\
\hline $09-08-93$ & 1815 & Steel tape & 18.99 & 0.01 & 15.65 & 419.45 & PM \\
\hline $09-15-83$ & 1615 & Steel tape & 18.89 & 0.01 & 15.55 & 419.55 & $M M$ \\
\hline $09-16-93$ & 1430 & E-tape & 18.89 & 0.02 & 15.55 & 419.55 & MM \\
\hline $09-29-93$ & 1631 & E-tape & 18.60 & 0.02 & 15.26 & 419.84 & PM \\
\hline $10-08-93$ & 1640 & E-tape & 18.62 & 0.02 & 15.28 & 419.82 & PM \\
\hline $10-14-93$ & 1257 & E-tape & 18.81 & 0.02 & 15.47 & 419.63 & $\mathrm{PM}$ \\
\hline $10-19-93$ & 1255 & Steel tape & 18.91 & 0.01 & 15.57 & 419.53 & MM \\
\hline
\end{tabular}

Site ID:

645112147491601 Local Number: $\overline{\text { FC00100106DBCA1 }}$ 


\begin{tabular}{|c|c|c|c|c|c|c|c|c|}
\hline 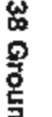 & \multicolumn{4}{|c|}{$\begin{array}{l}\text { NW101 GROUND-WATER OBSERVATION WELL } \\
\text { Near Power Plant }\end{array}$} & \multicolumn{2}{|c|}{ (Continued) } & \multirow{2}{*}{$\begin{array}{l}\text { Site 10: } \\
\text { Local Number: } \\
\text { WS elevation }\end{array}$} & \multirow{2}{*}{$\begin{array}{r}\frac{645 \$ 12 \nmid 47491601}{\text { FC00100106DBCA1 }} \\
\text { Remarks }\end{array}$} \\
\hline & Date & Time & Method & $\overline{\mathrm{MP}}$ to WS & Error & LS to WS & & \\
\hline 7 & $11-04-93$ & 1558 & $\bar{E}$-tape & $1 \overline{9.36}$ & 0.02 & 16.02 & $41 \overline{9.08}$ & $\mathrm{PM}$ \\
\hline a & $11-17-93$ & 1541 & Steel tape & 19.78 & 0.01 & 16.44 & 418.66 & MM \\
\hline 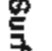 & $\{2-01-93$ & 1502 & E-tape & 19.92 & 0.02 & 16.58 & 418.52 & PM \\
\hline 蛋 & $12-07-93$ & 1431 & Steel tape & 19.89 & 0.01 & 16.55 & 418.55 & PM \\
\hline 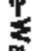 & $12-08-93$ & 1602 & E-tape & 19.99 & 0.02 & 16.65 & 418.45 & PM \\
\hline & $12-09-93$ & 0956 & Steel tape & 19.96 & 0.01 & 16.62 & 418.48 & PM \\
\hline 览 & $12-22-93$ & 1144 & E-tape & 20.04 & 0.02 & 16.70 & 418.40 & MM \\
\hline 总 & $01-05-94$ & 0940 & E-tape & 20.13 & 0.02 & 16.79 & 418.31 & PM \\
\hline 产 & $01-19-94$ & 1226 & Steel tape & 20.40 & 0.01 & 17.06 & 418.04 & MM \\
\hline$\frac{6}{5}$ & $02-03-94$ & 1451 & Steel tape & 20.74 & 0.01 & 17.40 & 417.70 & $P M$ \\
\hline 富 & $02-24-94$ & 1320 & Steel tape & 21.02 & 0.01 & 17.68 & 417.42 & MM \\
\hline & $03-09-94$ & 1610 & Stoel tape & 21.05 & 0.01 & 17.71 & 417.39 & PM \\
\hline & $03-16-94$ & $\uparrow 424$ & Steel tape & 21.10 & 0.01 & 17.76 & 417.34 & MM \\
\hline$\vec{z}$ & $03-30-94$ & 1215 & Steel tape & 21.21 & 0.01 & 17.87 & 417.23 & PM \\
\hline $\mathrm{g}$ & $04-2\}-94$ & 1330 & Steel tape & 21.04 & 0.01 & 17.70 & 417.40 & $M M$ \\
\hline Z & $05-04-94$ & 1314 & E-tape & 20.55 & 0.02 & 17.21 & 417.89 & PAA \\
\hline 7 & $06-03-94$ & 0841 & Steel tape & 20.52 & 0.01 & 17.18 & 417.92 & PM \\
\hline 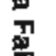 & $06-09-94$ & 1343 & Stecl tape & 20.64 & 0.01 & 17.30 & $4 \uparrow 7.80$ & $P M$ \\
\hline$\frac{\pi}{7}$ & $06-15-94$ & 1030 & Steol tape & 20.68 & 0.01 & 17.34 & 417.76 & $M M$ \\
\hline & $06-21-94$ & 1709 & Steel tape & 20.44 & 0.01 & 17.10 & 418.00 & PM \\
\hline & $07-01-84$ & 1545 & Steel tape & 19.49 & 0.01 & 16.15 & 418.95 & $P M$ \\
\hline & $07-15-94$ & 0844 & Steel tape & 19.95 & 0.01 & 16.61 & 418.49 & $M A$ \\
\hline 要 & $07-21-94$ & 1630 & Steel tape & 18.90 & 0.01 & 15.56 & 419.54 & PM \\
\hline & $08-03-94$ & 1331 & Steel tape & 18.97 & 0.01 & 15.63 & 419.47 & PM \\
\hline & $08-12-94$ & 1539 & Steel tape & 18.84 & 0.01 & 15.50 & 419.60 & $P M$ \\
\hline & $08-15-94$ & 1214 & Steel tape & 18.75 & 0.01 & 15.41 & 419.69 & MM \\
\hline & $09-08-94$ & 1740 & Steel tape & 19.00 & 0.01 & 15.66 & 419.44 & $P M$ \\
\hline & $09-15-94$ & 1249 & Steel tape & 19.21 & 0.01 & 15.87 & $4 \longdiv { 9 . 2 3 }$ & MM \\
\hline & $09-22-94$ & 1131 & Steel tape & 19.51 & 0.01 & 16.17 & 418.93 & $\mathrm{PM}$ \\
\hline & $09-29-94$ & 1238 & Steel tape & 19.82 & 0.01 & 16.48 & 418.62 & PM \\
\hline & $10-20-94$ & 1321 & Steed tape & 20.49 & 0.01 & 17.15 & 417.95 & MM \\
\hline
\end{tabular}




\begin{tabular}{|c|c|c|c|c|c|c|c|}
\hline $\begin{array}{l}\text { MW101 GRe } \\
\text { Near Power }\end{array}$ & WATE & SERVATIC & WELL & Contint & & $\begin{array}{l}\text { Site ID: } \\
\text { Local Number: }\end{array}$ & $\begin{array}{r}645112147491601 \\
\text { FC00100106D8CA1 } \\
\end{array}$ \\
\hline Date & Time & Method & MP to WS & Error & LS to WS & WS elevation & Remarks \\
\hline $10-25-94$ & 1342 & Steel tape & 20.61 & 0.01 & 17.27 & 417.83 & $\mathrm{PM}$ \\
\hline $11-17-94$ & 1205 & Steel tape & 21.10 & 0.01 & 17.76 & 417.34 & MM \\
\hline $12-02-94$ & $\uparrow 412$ & E-tape & NA & 0.02 & NA & NA & $\mathrm{PM}$ \\
\hline $12-23-94$ & 1503 & Steel tape & 21.09 & 0.01 & 17.75 & 417.35 & MM \\
\hline 0$\}-18-85$ & 1600 & Steel tape & NA & 0.01 & NA & NA & $M M$ \\
\hline $02-16-95$ & 1455 & Steel tape & $N A$ & 0.01 & NA & NA & MM \\
\hline $02-21-95$ & 1632 & Steel tape & 21.58 & 0.01 & 18.24 & 416.86 & $\mathbf{P T}$ \\
\hline $02-22-95$ & 1115 & Steel tape & 21.68 & 0.01 & 18.34 & 416.76 & PT \\
\hline $02-22-95$ & 1115 & Steel tape & 21.68 & 0.01 & 18.34 & 416.76 & PT \\
\hline $02-23-95$ & 1150 & Steel tape & NA & 0.01 & $\mathrm{NA}$ & NA & $\mathrm{PT}$ \\
\hline $02-24-95$ & 1000 & Steel tape & 22.21 & 0.01 & 18.87 & 416.23 & $P T$ \\
\hline $02-26-95$ & 1514 & Steel tape & 22.45 & 0.01 & 19.11 & 415.99 & PT \\
\hline $02-27-95$ & 0959 & Steel tape & 22.48 & 0.01 & 19.14 & 415.96 & PT \\
\hline $03-01-95$ & 0910 & Steel tape & 22.63 & 0.01 & 19.29 & 415.81 & PT \\
\hline $03-02-95$ & 1253 & Steel tape & $22.4 \uparrow$ & 0.01 & 19.07 & 416.03 & PT \\
\hline $03-06-95$ & 1025 & Steel tape & 22.69 & 0.01 & 19.35 & 415.75 & $\mathrm{PT}$ \\
\hline $03-08-95$ & 1456 & Steel tape & 22.68 & 0.01 & 19.34 & 415.76 & PT \\
\hline $03-13-95$ & 1054 & Steet tape & 22.78 & 0.01 & 19.44 & 415.66 & PT \\
\hline $03-20-95$ & 1434 & Steel tape & 21.86 & 0.01 & 18.52 & 416.58 & MM \\
\hline $04-15-95$ & 1109 & Steel tape & 21.00 & 0.01 & 17.66 & 417.44 & MM \\
\hline $05-17-95$ & 1000 & Steel tape & 20.02 & 0.01 & 16.68 & 418.42 & MM \\
\hline $05-26-95$ & 1345 & Steel tape & 19.77 & 0.01 & 16.43 & 418.67 & $P M$ \\
\hline $07-18-95$ & 1049 & Steel tape & 18.88 & 0.01 & 15.54 & 419.56 & $M M$ \\
\hline $08-16-95$ & 1519 & Steel tape & 18.24 & 0.01 & 14.90 & 420.20 & MM \\
\hline $09-14-95$ & 1519 & E-tape & 17.57 & 0.02 & 14.23 & 420.87 & MM \\
\hline $10-17-95$ & 1257 & E-tape & 18.76 & 0.02 & 15.42 & 419.68 & MM \\
\hline
\end{tabular}




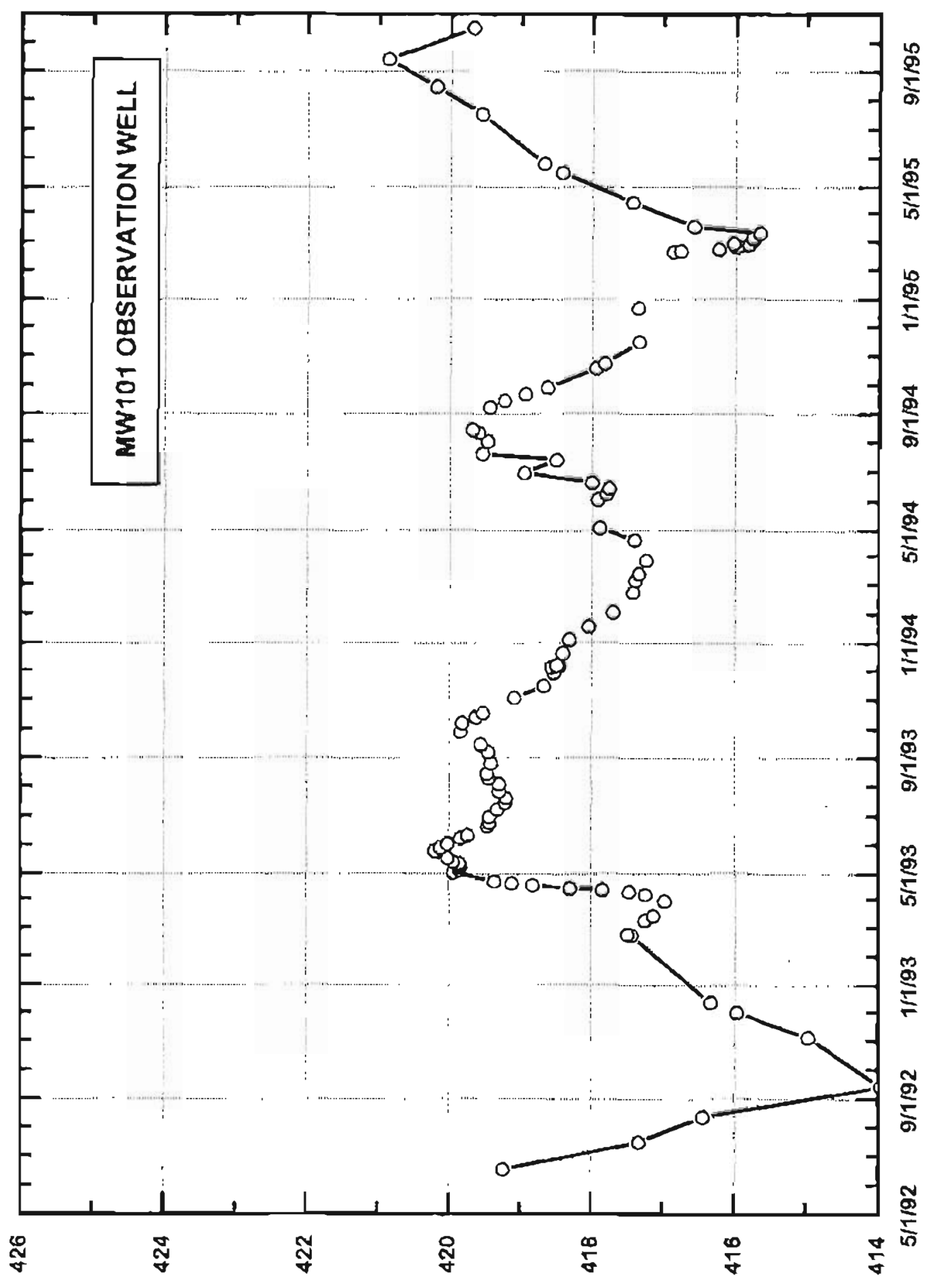

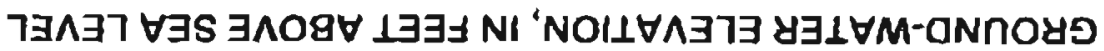


MWSO2 GROUND-WATER OBSERVATION WELL

West of gas pump

All measurements in feet

Depth to bottom of well from MP :

Depth from TOC to top of SI :

Depth trom TOC to bottom of SI:

Land Surface Datum :

\begin{tabular}{c|c} 
Feet & Elevation \\
\hline 24.6 & 407.6 \\
5.1 & 427.1 \\
24.5 & 407.7 \\
& 432.5
\end{tabular}

Datum corrections, reference survey notes in site folders

\begin{tabular}{c|c} 
Date & MP elevation (feel above sea level) \\
\hline $07-01-92$ & 432.13
\end{tabular}
Site ID:

Local Number:

$\begin{array}{r}645115147485001 \\ \hline \text { FC00100106DACA1 }\end{array}$

LS, land surface

$M M$, mass measurement

$M P$, measuring point

$N A$, not available

PM, partlal measurement

SI, screened interval

TOC, top of casing

WS, water surface

\begin{tabular}{|c|c|c|c|c|c|c|c|}
\hline Date & Time & Method & MP to WS & Error & LS to WS & WS elevation & Remarks \\
\hline $07-17-92$ & $15 \overline{42}$ & Steel tape & $15 \overline{94}$ & $\overline{0} .0 \overline{1}$ & 16.31 & $4 \overline{16.19}$ & $\mathrm{MM}$ \\
\hline $08-13-92$ & 2153 & Steel tape & 16.86 & 0.01 & 17.23 & 415.27 & MM \\
\hline $09-14-92$ & 1925 & Steel tape & NA & 0.01 & NA & NA & PM, blocked inner casing \\
\hline $12-01-92$ & 1125 & Steel tape & 15.47 & 0.01 & 15.84 & 416.66 & MM \\
\hline $12-14-92$ & 0951 & Steel tape & 15.05 & 0.01 & $\$ 5.42$ & 417.08 & PNH \\
\hline $02-19-93$ & 1154 & Steel tape & 13.94 & 0.01 & 14.31 & 418.19 & $\mathrm{MM}$ \\
\hline $02-25-93$ & 1627 & Steel tape & 13.87 & 0.01 & 14.24 & 418.26 & $M N$ \\
\hline $03-12-93$ & 1115 & Steel tape & 14.19 & 0.01 & 14.56 & 417.94 & MM \\
\hline $04-16-93$ & 1432 & Steel tape & 13.22 & 0.01 & 13.59 & 418.91 & MM \\
\hline $06-11-93$ & 1127 & Steel tape & 11.65 & 0.01 & 12.02 & 420.48 & MM \\
\hline $07-16-93$ & $\uparrow 253$ & Steel tape & $\$ 2.14$ & 0.01 & 12.51 & 419.99 & MM \\
\hline $08-16-93$ & 0855 & Steel tape & 11.94 & 0.01 & 12.31 & 420.19 & MM \\
\hline $09-15-93$ & 1636 & E-tape & 11.79 & 0.02 & 12.16 & 420.34 & MM \\
\hline
\end{tabular}




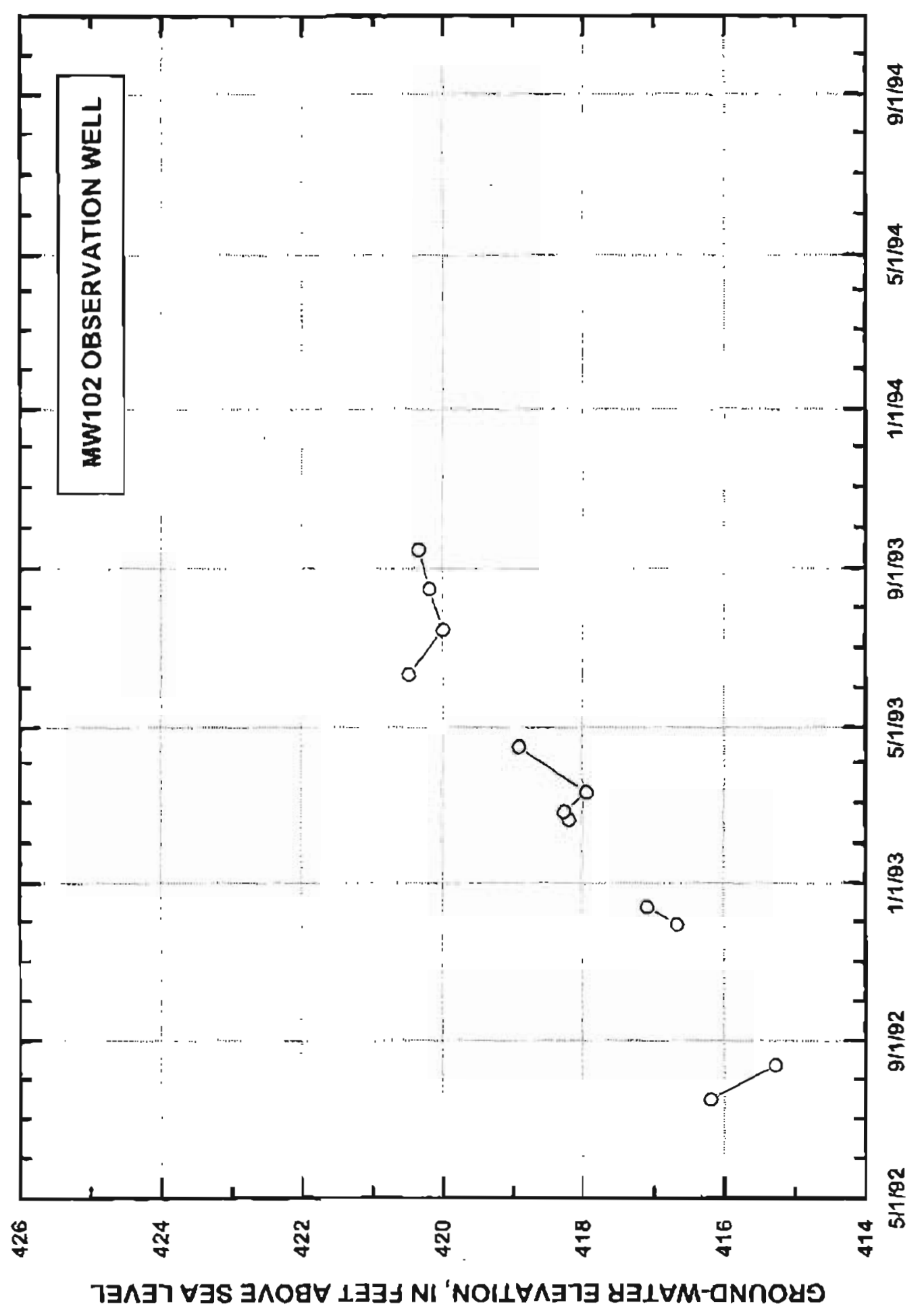


Site ID:

Local Number

645117147484304

(1)

\begin{tabular}{c|c} 
Feet & Elevation \\
\hline NA & NA \\
NA & NA \\
NA & NA \\
& 432.5
\end{tabular}

All measurements in feet

Depth to bottom of well from MP :

Depth from TOC to top of $S \mid$ :

Depth from TOC to bottom of S1:

Land Surface Datum:

Datum corrections, reference survey notes in site folders

$$
\begin{array}{c|rc}
\text { Date } & \text { MP elevation (feet above sea level) } \\
\hline 07-01-92 & 432.02 \\
11-03-92 & 432.02 \quad \text { Updated MP }
\end{array}
$$

\begin{tabular}{cccccccc}
\hline Date & Time & Method & MP to WS & Eror & LS to WS & WS etevation & Remarks \\
\hline $07-15-94$ & 0844 & Steel tape & 11.28 & $\overline{0} .01$ & $11.7 \overline{6}$ & $4 \overline{20.74}$ & MM \\
$09-15-94$ & 1502 & Steel tape & 11.90 & 0.01 & 12.38 & 420.12 & MM \\
\hline
\end{tabular}

LS, land surface

MAh, mass measurement

$M P$, measuring point

NA, not avajlable

PM, partial measurement

Sl, screened interval

TOC, top of casing

WS, water surface 


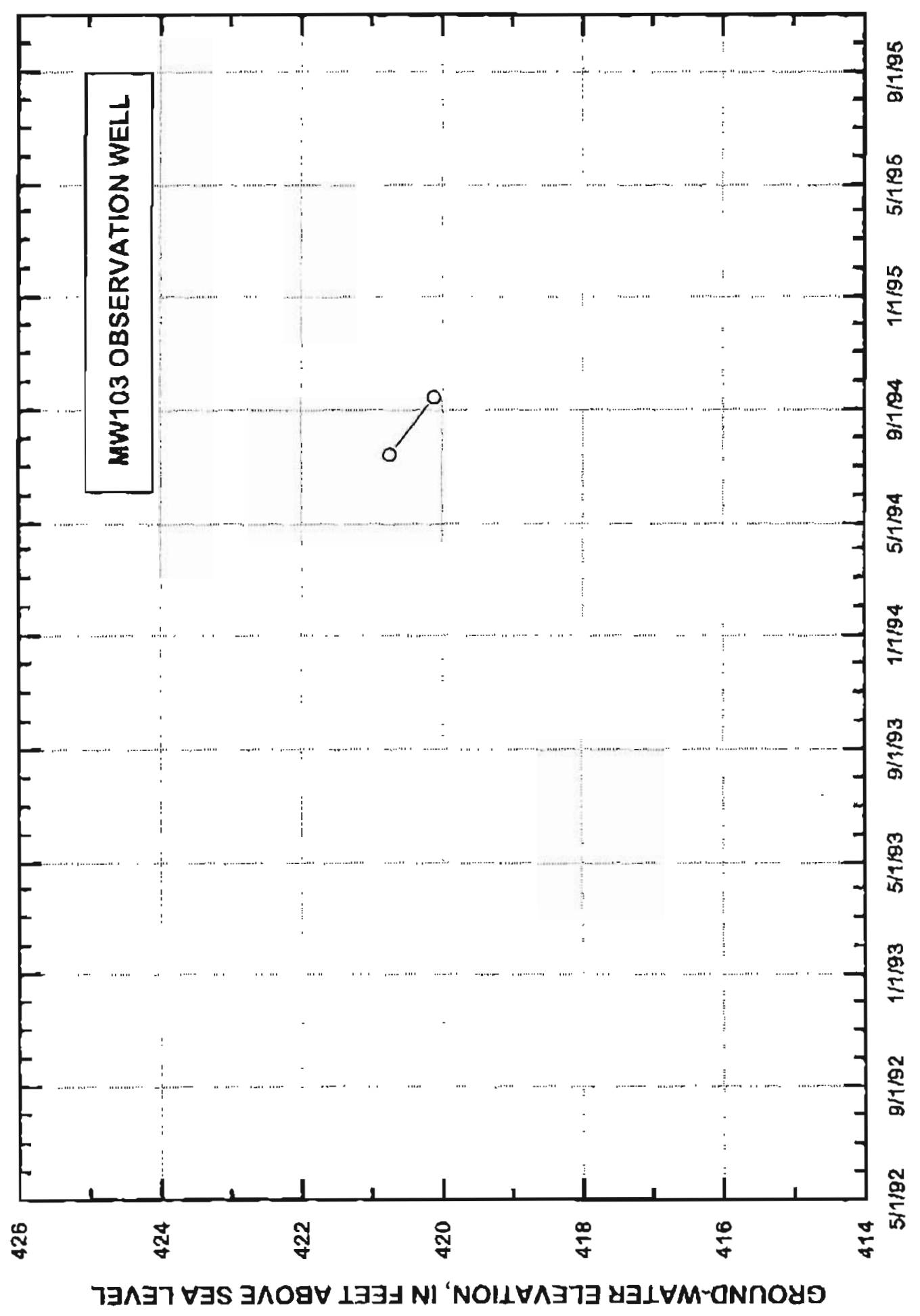




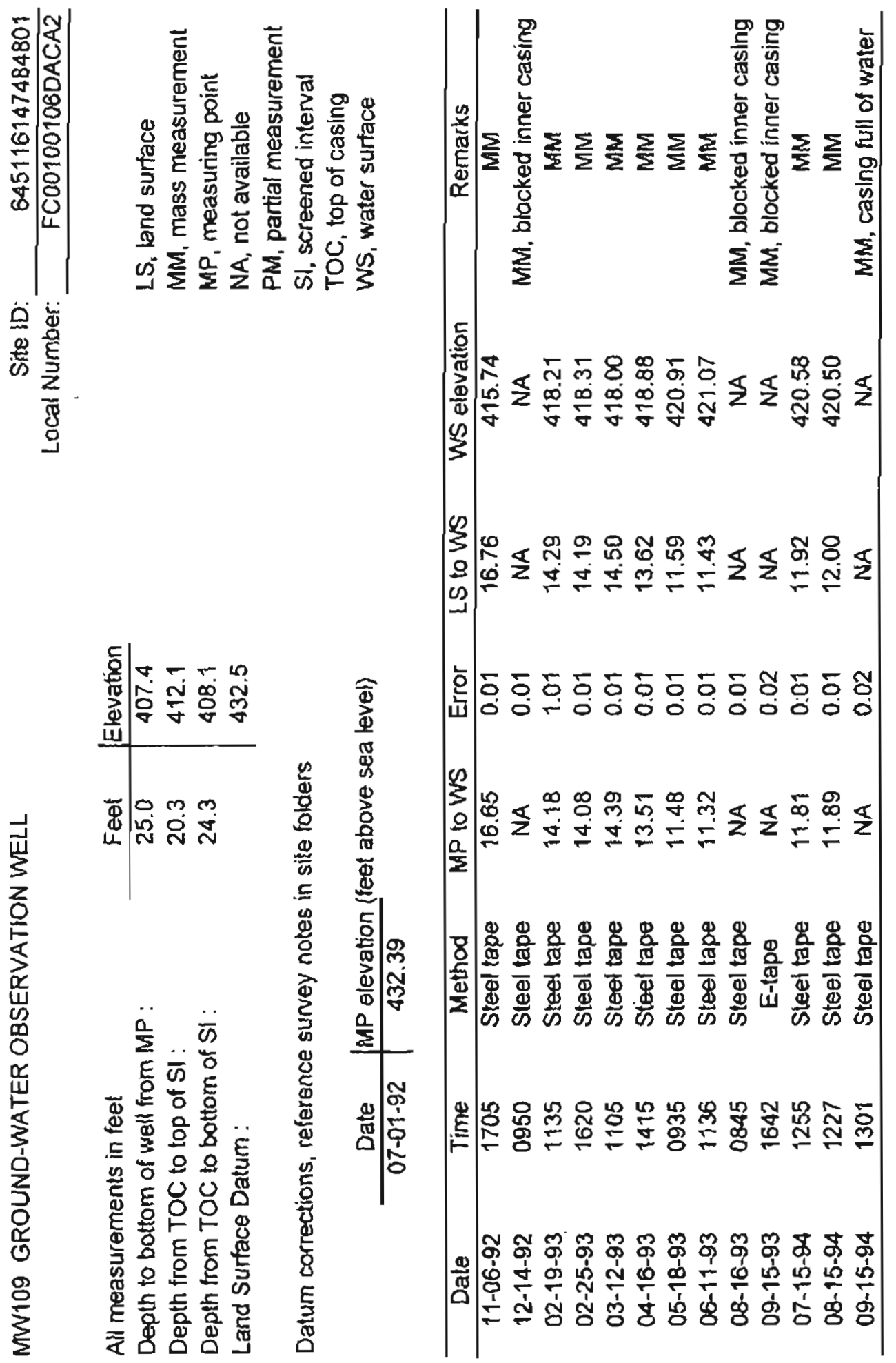




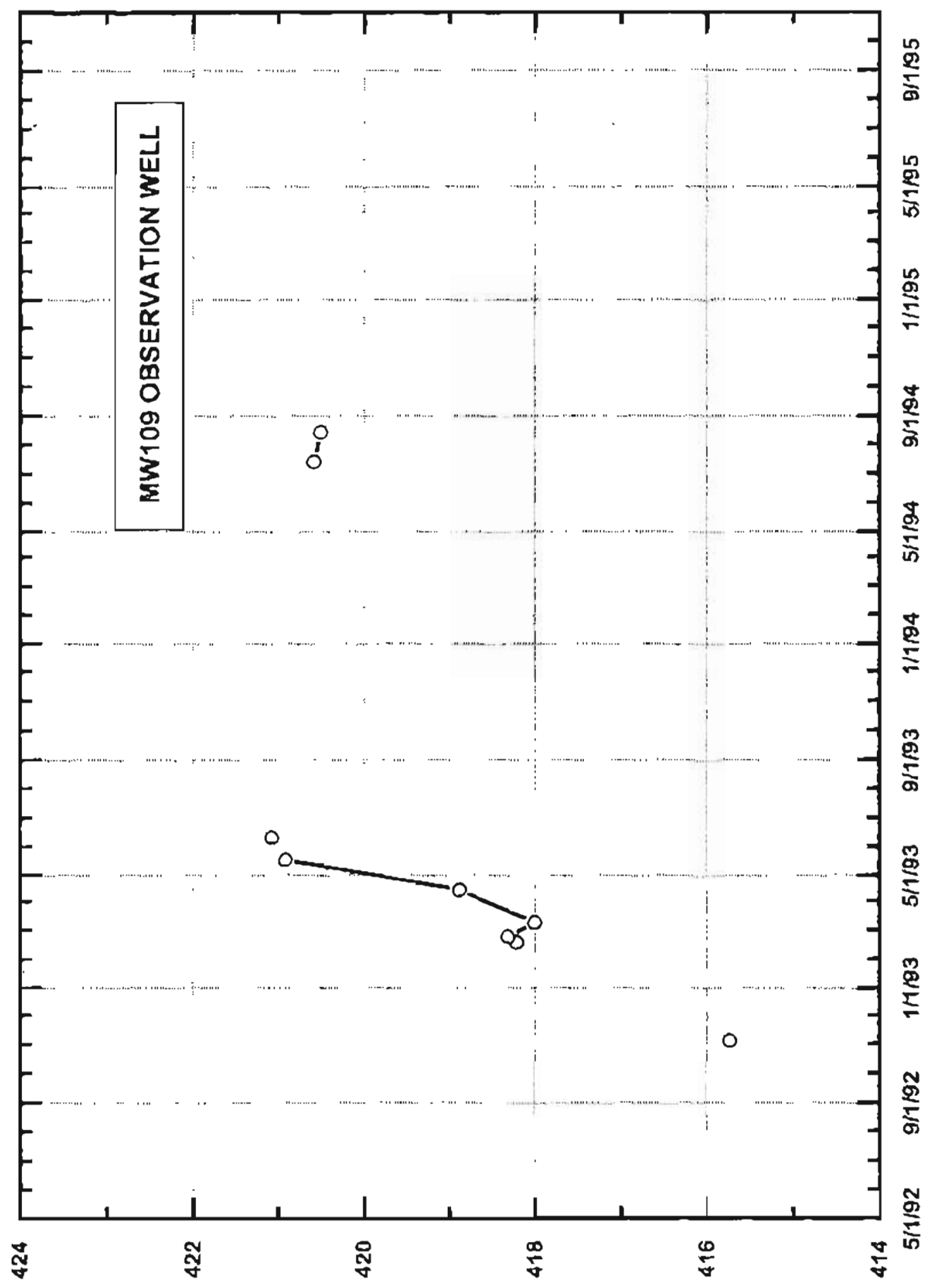

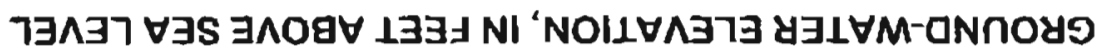


Site ID: Local Number:

\begin{tabular}{c|c} 
Feet & Elevation \\
\hline 23.8 & 408.3 \\
19.7 & 412.3 \\
24.2 & 407.9 \\
& 432.5
\end{tabular}

All measurements in feet Depth to bottom of well from MP : Depth from TOC to top of Si :

Deptin from TOC to bottom of SI :

Land Surface Datum:

Datum corrections, reference survey notes in stle folders

\begin{tabular}{c|cc} 
Date & MP Elevation (feet above sea level) \\
\hline $07-01-92$ & 432.43 & \\
$11-03.92$ & $432.02 \quad$ Updated MP
\end{tabular}

$645117\{47484801$ FC00100106DACA3

LS, and surface MM, mass measurement $M P$, measuring point NA, not available PM, partial measurement $\mathrm{SI}$, screened interval TOC, top of casing WS, water surface

\begin{tabular}{|c|c|c|c|c|c|c|c|}
\hline Date & Time & Method & $\mathrm{MP}$ to WS & Error & $\mathrm{LS}$ to WS & WS elevation & Remarks \\
\hline $07-17.92$ & 1624 & Steel tape & NA & 0.01 & NA & $\overline{\mathrm{NA}}$ & MM, Blocked \\
\hline $08-13-92$ & 2203 & Steel tape & NA & 0.01 & NA & NA & MM, Blocked \\
\hline $05-18-93$ & 1316 & Steel tape & 11.44 & 0.01 & 11.92 & 420.58 & MM \\
\hline $06-11-93$ & 1152 & Steel tape & 11.82 & 0.01 & 12,30 & 420.20 & MN \\
\hline $08-16-93$ & 0853 & Steel tape & 12.13 & 0.01 & 12.61 & 419.89 & $M M$ \\
\hline $09-15-93$ & 1645 & E-tape & 11.97 & 0.02 & 12.45 & 420.05 & $M M$ \\
\hline $07-15-94$ & 1039 & Steel tape & 11.79 & 0.01 & 12.27 & 420.23 & MM \\
\hline $08-15-94$ & 1232 & Steel tape & 11.76 & 0.01 & 12.24 & 420.26 & MM \\
\hline $09-\frac{1}{5}-94$ & 1308 & Steel tape & $12.3 \mathrm{~B}$ & 0.01 & 12.86 & 419.64 & $M M$ \\
\hline
\end{tabular}




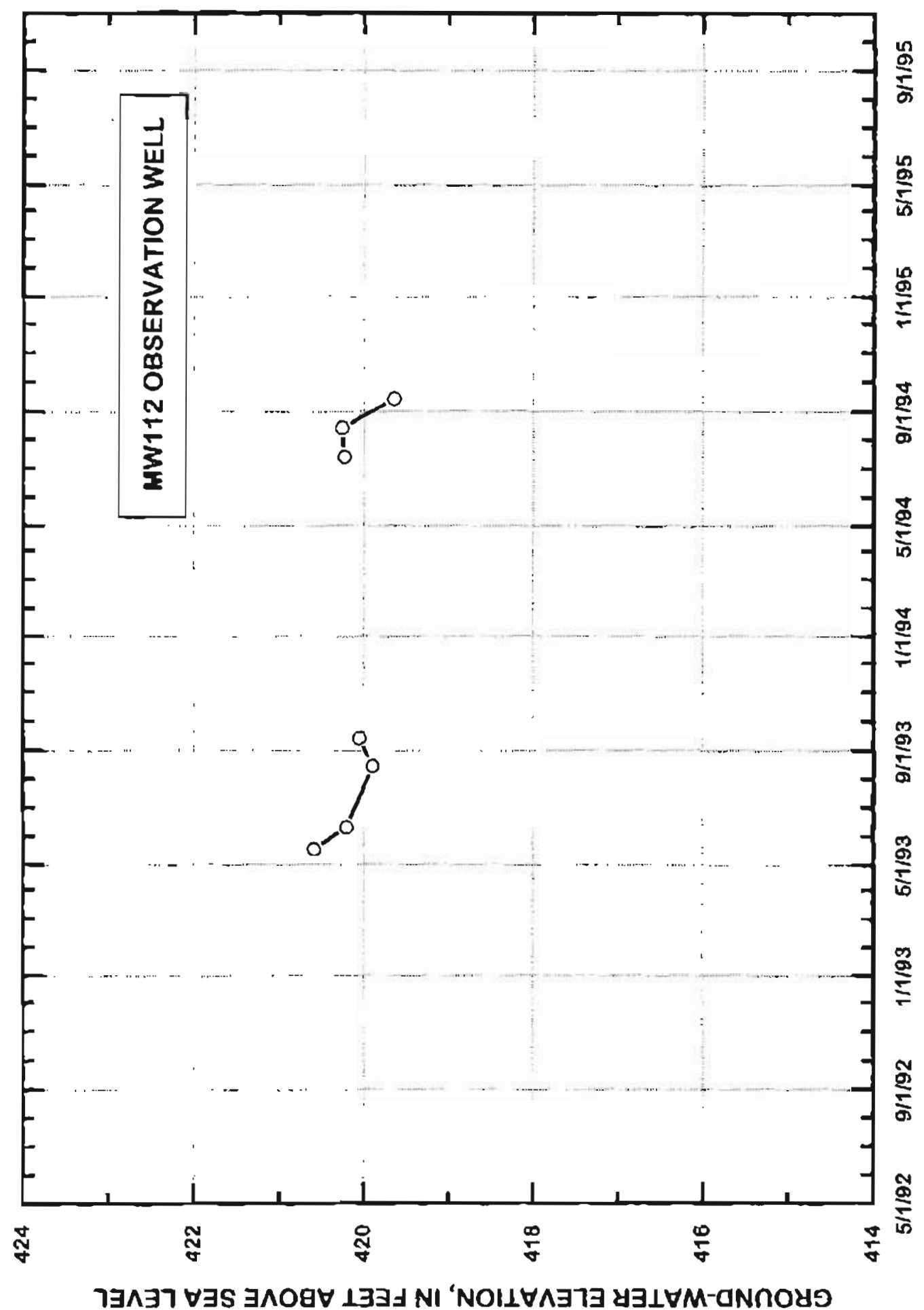


MW113 GROUND-WATER OBSERVATION WELL

Near UAF gravel pit

All measurements in fee:

Depth to bottom of well from MP :

Depti fron TOC to top of $\mathrm{SI}$ :

Depth from TOC to bottom of $\$$ :

Land Surface Datum:

\begin{tabular}{c|c} 
Feet & Elevation \\
\hline 26.9 & 409.1 \\
19.1 & 417.0 \\
23.1 & 412.9 \\
& 432.7
\end{tabular}

Datum corrections, reference survey notes in site folders

$$
\begin{array}{c|c}
\text { Date } & \text { MP Elevation (feet above sea (evel) } \\
\hline 07-01-92 & 436.03
\end{array}
$$

Site ID:

Local Number.

645113147485301 FC00100106DACC1

LS, land surface

$M M$, mass measurement

MP, measuring point

NA, not available

PN, partial measurement

SI, screened interval

TOC, top of casing

WS, water surface

\begin{tabular}{|c|c|c|c|c|c|c|c|}
\hline Date & Time & Method & MP to WS & Error & LS to WS & WS elevation & Remarks \\
\hline $07-17-92$ & 1531 & Steel tape & 19.70 & 0.01 & 16.37 & 416.33 & MN \\
\hline $08-13-92$ & 2131 & Steel tape & 20.75 & 0.01 & 17.42 & 415.28 & $\mathrm{MM}$ \\
\hline $09-14-92$ & 1917 & Steel tape & 23.38 & 0.01 & 20.05 & 412.65 & PM \\
\hline $11-06-92$ & 1645 & Steel tape & 19.56 & 0.01 & 16.23 & 416.47 & MM \\
\hline $12-01-92$ & 1158 & Steel tape & 19.62 & 0.01 & 16.29 & 416.41 & $M M$ \\
\hline $12-14-92$ & 1010 & Steel tape & 19.21 & 0.01 & 15.88 & 416.82 & MM \\
\hline $02-26-93$ & 1455 & Steel tape & NA & 0.01 & NA & NA & PM, Blocked inner casing \\
\hline $04-16-93$ & 1445 & Steel tape & 14.27 & 0.01 & 10.94 & 421.76 & MM \\
\hline $05-18-93$ & 0945 & Steet tape & 15.46 & 0.01 & 12.13 & 420.57 & MM \\
\hline $06-11-93$ & 1138 & Steel tape & 15.88 & 0.01 & 12.55 & 420.15 & MM \\
\hline $07-46-93$ & 1216 & Steel tape & 16.32 & 0.01 & 12.99 & 419.71 & MM \\
\hline $08-16-93$ & 0900 & Steel tape & 16.10 & 0.01 & 12.77 & 419.93 & MM \\
\hline $09-15-93$ & 1652 & Steel tape & 15.96 & 0.01 & 12.63 & 420.07 & MM \\
\hline $10-19-93$ & 1245 & Steel tape & 16.00 & 0.01 & 12.67 & 420.03 & MM \\
\hline $11-16-93$ & 1500 & Steel lape & 16.79 & 0.01 & 13.46 & 419.24 & $M M$ \\
\hline $12-22-93$ & 1103 & E-tape & 17.11 & 0.02 & 13.78 & 418.92 & MM \\
\hline $01-19-94$ & 1219 & Steel tape & 17.38 & 0.01 & 14.05 & 418.65 & MM \\
\hline $02-24-94$ & 1312 & Steel tape & 18.03 & 0.01 & 14.70 & 418.00 & MM \\
\hline $03-\uparrow 6-94$ & 1200 & Steel tape & 18.15 & 0.01 & 14.82 & 417.88 & $M A$ \\
\hline $04-21-94$ & 1410 & Steel tape & 18.11 & 0.01 & 14.78 & 417.92 & MM \\
\hline
\end{tabular}




\begin{tabular}{|c|c|c|c|c|c|c|c|}
\hline \multicolumn{4}{|c|}{$\begin{array}{l}\text { MW113 GROUND-WATER OBSERVATION WELL } \\
\text { Near UAF gravel pit }\end{array}$} & \multicolumn{2}{|c|}{ Continued) } & \multirow{2}{*}{$\begin{array}{r}\text { Site ID: } \\
\text { Local Number: } \\
\text { WS elevation }\end{array}$} & \multirow{2}{*}{$\begin{array}{r}645113147485301 \\
\text { FC00100106DACC1 } \\
\text { Remarks }\end{array}$} \\
\hline Date & Time & Method & MP to WS & Error & LS to WS & & \\
\hline $06-15-94$ & 0906 & Steel tape & 17.68 & 0.01 & 14.35 & 418.35 & $\mathrm{MM}$ \\
\hline $07-15-94$ & 1012 & Steel tape & 15.79 & 0.01 & 12.46 & 420.24 & MM \\
\hline $08-15-94$ & 1222 & Steel tape & 15.85 & 0.01 & 12.52 & 420.18 & MM \\
\hline $09-15-94$ & 1257 & Steel tape & 16.42 & 0.01 & 13.09 & 419.61 & MM \\
\hline $09-22-94$ & $\$ 125$ & Steel tape & 16.70 & 0.01 & 13.37 & 419.33 & MM \\
\hline $04-15-95$ & 1103 & Steet tape & 18.47 & 0.01 & 14.84 & 417.86 & $M M$ \\
\hline $05-17-95$ & 1005 & Steel tape & 17.09 & 0.01 & 13.76 & 418.94 & MM \\
\hline $06-15-95$ & 1443 & E-tape & 17.25 & 0.02 & 13.92 & 418.78 & MM \\
\hline $07-17-95$ & 1731 & Steel tape & 15.97 & 0.01 & 12.64 & 420.06 & MM \\
\hline $08-15-95$ & 1331 & E-tape & 16.22 & 0.02 & 12.89 & 419.81 & $M M$ \\
\hline $09-14-95$ & 1515 & E-tape & 15.16 & 0.02 & 11.83 & 420.87 & MM \\
\hline
\end{tabular}




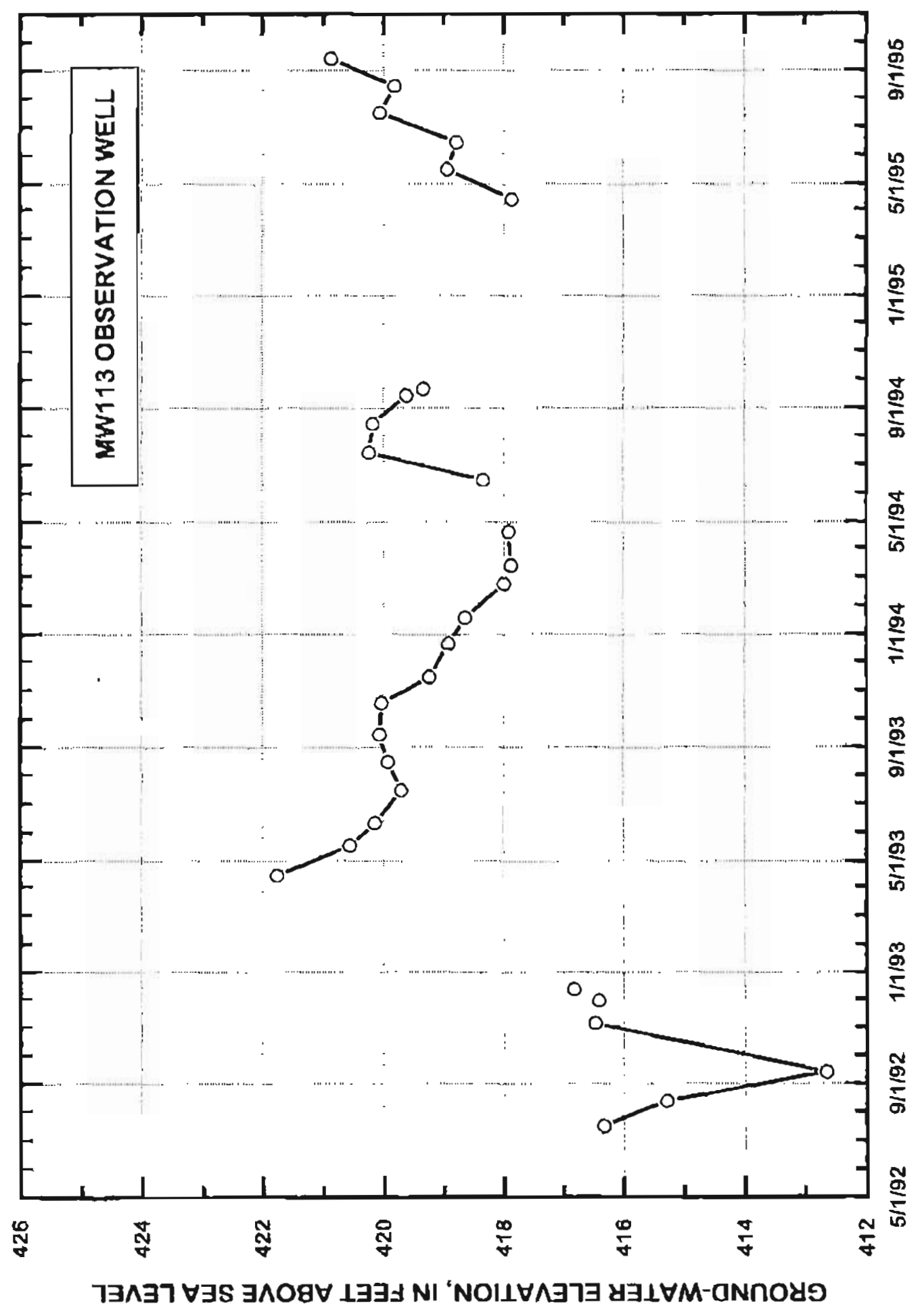


Site ID: Local Number

\begin{tabular}{c|c} 
Feet & Elevation \\
\hline 18.9 & 414.0 \\
7.6 & 425.3 \\
17.6 & 415.3 \\
& 431.4
\end{tabular}

All measurements in feet

Depth to bottom of well from MP :

Depth from TOC to top of $\$ 1$ :

Depth from TOC to boltom of S1:

Land Surface Datum:

Datum corrections, reference suney notes in site folders

\begin{tabular}{c|c} 
Date & MP Elevation (feet above sea level) \\
\hline $07-01-92$ & 432.90 \\
$07-31-92$ & 432.90
\end{tabular}

$\frac{645121147484001}{\text { FC00100106DAAB1 }}$

\section{LS, land surtace} $M M$, mass measurement $M P$, measuring point NA, not available PM, partial measurement S1, screened interval TOC, top of casing WS, water surface

\begin{tabular}{|c|c|c|c|c|c|c|c|}
\hline Date & Time & Method & MP to WS & Error & LS to WS & WS elevation & Remarks \\
\hline $07-17-92$ & 1659 & Steel tape & 17.70 & 0.01 & 16.20 & 415.20 & $M M$ \\
\hline $08-14-92$ & 1219 & Steel tape & NA & 0.01 & NA & NA & PM, dry \\
\hline $09-14-92$ & 1957 & Steel tape & NA & 0.01 & NA & NA & $P M$, dry \\
\hline $11-06-92$ & 0930 & Steel tape & 17.50 & 0.01 & 16.00 & 415.40 & MM \\
\hline $12-01-92$ & 1036 & Steel tape & 16.48 & 0.01 & 14.98 & 416.42 & $M M$ \\
\hline $12-14-82$ & 0846 & Steel lape & 16.06 & 0.01 & 14.56 & 416.84 & $M M$ \\
\hline $02-12-93$ & 1445 & Sted tape & 14.82 & 0.01 & 13.32 & 418.08 & PM \\
\hline $02-19-93$ & 1035 & Steel tape & 14.80 & 0.01 & 13.30 & 418.10 & MM \\
\hline $02-25-93$ & 1610 & Steel tape & 14.71 & 0.01 & 13.21 & 418.19 & $M A M$ \\
\hline $03-12-93$ & 1123 & Steel tape & 15.05 & 0.01 & 13.55 & 417.95 & MN \\
\hline $03-17-93$ & 1508 & Steel tape & 15.07 & 0.01 & 13.57 & 417.83 & PM \\
\hline $04-02-93$ & 1626 & Steel tape & 15.22 & 0.01 & 13.72 & 417.68 & PM \\
\hline $04-09-93$ & 1418 & Steel tape & 15.00 & 0.01 & 13.50 & 417.90 & PM \\
\hline $04-16-93$ & 1350 & Steel tape & 14.48 & 0.01 & 12.98 & 418.42 & $M M$ \\
\hline $05-03-93$ & 1156 & Steel tape & 12.54 & 0.01 & $\{1.04$ & 420.36 & PM \\
\hline $05-05-93$ & 1134 & Steel tape & 12.37 & 0.01 & 10.87 & 420.53 & PM \\
\hline $05-06-93$ & 1641 & Steel tape & 12.33 & 0.01 & 10.83 & 420.57 & $P M$ \\
\hline $05-14-93$ & 0945 & Steel tape & 12.04 & 0.01 & 10.54 & 420.86 & PM \\
\hline
\end{tabular}


MW201 GROUND-WATER OBSERVATION WELL (Continued)

Site iD:

645121147484001

Local Number:

FC00100106DAAB1

\begin{tabular}{|c|c|c|c|c|c|c|c|}
\hline Date & Time & Method & MP to WS & Error & LS to WS & WS elevation & Remarks \\
\hline $05-18-93$ & 0907 & Steel tape & 11.99 & 0.01 & 10.49 & 420.91 & MA \\
\hline $06-11-93$ & 1138 & Steel tape & 12.42 & 0.01 & 10.92 & 420.48 & MM \\
\hline $06-30-93$ & 1903 & E-tape & 12.70 & 0.02 & 11.20 & 420.20 & $P M$ \\
\hline $07-16-93$ & 1228 & Steel tape & 12.95 & 0.02 & 11.45 & 419.95 & MM \\
\hline $08-16.93$ & 0825 & Steel tape & 12.81 & 0.01 & 11.31 & 420.09 & $\mathrm{MM}$ \\
\hline $09-08-93$ & 1805 & Steel tape & $\{2.65$ & 0.01 & 11.15 & 420.25 & $\mathrm{PM}$ \\
\hline $09-15-93$ & 1553 & E-tape & 12.58 & 0.02 & 11.08 & 420,32 & MM \\
\hline $10-19-93$ & 1230 & Steel tape & 12.52 & 0.01 & $\uparrow 1.02$ & 420.38 & $\mathrm{MM}$ \\
\hline $11-16-93$ & 1555 & Steel tape & 13.27 & 0.01 & 11.77 & 419.63 & MM \\
\hline $12-22-93$ & 1037 & E-tape & 13.59 & 0.02 & 12.09 & 419.31 & $M M$ \\
\hline $04-19-94$ & 1201 & Steel tape & 13.93 & 0.01 & 12.43 & 418.97 & MM \\
\hline $02-24-94$ & 1146 & Steel tape & 14.58 & 0.01 & 13.08 & 418.32 & MM \\
\hline $03-16-94$ & 1129 & E-tape & $\{4.77$ & 0.02 & 13.27 & 418.13 & $\mathrm{MM}$ \\
\hline $04-21-94$ & 1307 & Steel tape & 14.89 & 0.01 & 13.39 & 418.01 & MM \\
\hline $06-15-94$ & 0836 & Steel lape & 14.32 & 0.01 & 12.82 & 418.58 & MM \\
\hline $07-15-94$ & 0946 & Steel tape & 12.40 & 0.01 & 10.90 & 420.50 & MM \\
\hline $08-15-94$ & 1243 & Steel tape & 12.50 & 0.01 & 11.00 & 420.40 & $M M$ \\
\hline $09-15-94$ & 1449 & Steel tape & 13.01 & 0.01 & 11.51 & 419.89 & MM \\
\hline $10-20-94$ & 1333 & Steel tape & 14.12 & 0.01 & 12.62 & 418.78 & $M M$ \\
\hline $11-17-94$ & 1210 & Steel tape & 14.68 & 0.01 & $\nmid 3.18$ & 418.22 & MM \\
\hline $01-18-95$ & 1615 & Steel tape & 14.87 & 0.01 & 13.37 & 418.03 & $M M$ \\
\hline $02-36-95$ & 3450 & Steel tape & 15.18 & 0.01 & 13.68 & 417.72 & MM \\
\hline $04-15-95$ & 1056 & Steel tape & 15.17 & 0.01 & 13.67 & 417.73 & $M M$ \\
\hline $05-17-95$ & 1009 & Steel tape & 13.69 & 0.01 & 12.19 & 419.21 & MM \\
\hline $06-15-95$ & 1433 & E-tape & 13.80 & 0.02 & 12.30 & 419.10 & MM \\
\hline $07-17-95$ & 1722 & Steel tape & 12.68 & 0.01 & 11.18 & 420.22 & MM \\
\hline $08-15-95$ & 1325 & E-tape & 12.87 & 0.02 & 11.37 & 420.03 & MM \\
\hline $09-14-95$ & 1505 & E-tape & 11.73 & 0.02 & 10.23 & 421.17 & MM \\
\hline $10-17-95$ & 1240 & E-tape & 12.44 & 0.02 & 10.94 & 420.46 & MM \\
\hline
\end{tabular}




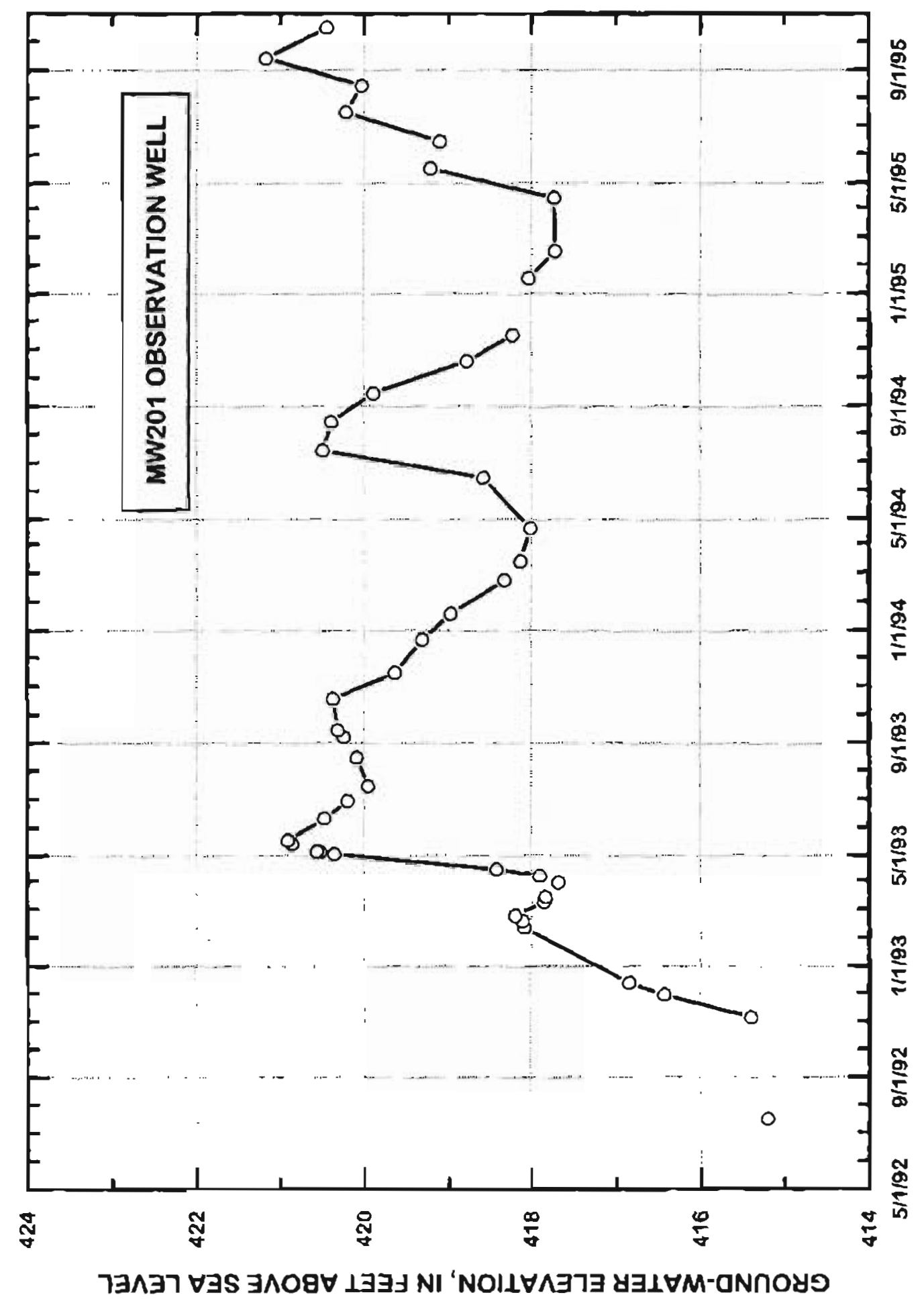

WATER-ELEVATION TABLES AND HYDROGRAPHS 57 

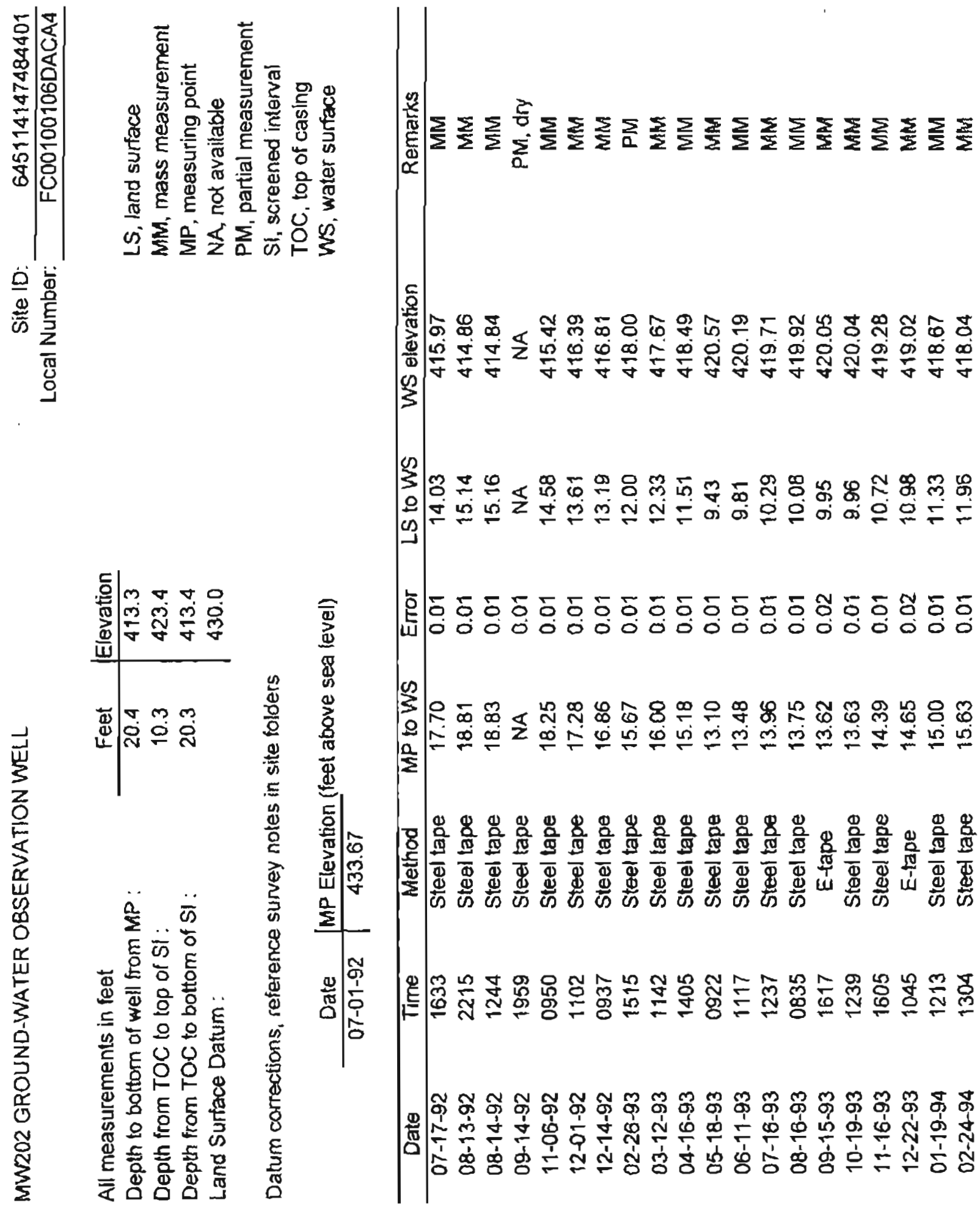
MW202 GROUND-WATER OBSERVATION WELL (COntinuEd)

Site 10:

645114147484401

Local Number:

FC $\overline{C 00100106 D A C A 4}$

\begin{tabular}{cccccccc}
\hline Date & Time & Method & MP to WS & Error & LS to WS & WS elevation & Rernarks \\
\hline $03-16-94$ & 1149 & E-tape & 15.78 & 0.02 & 12.11 & 417.89 & MM \\
$04-21-94$ & 1331 & Steel tape & 15.83 & 0.01 & 12.16 & 417.84 & MM \\
$06-15-94$ & 0900 & Steel tape & 15.37 & 0.01 & 11.70 & 418.30 & MM \\
$07-15-94$ & 0954 & Steel tape & 13.39 & 0.01 & 9.72 & 420.28 & MM \\
$08-15-94$ & 1248 & Steel tape & 13.56 & 0.01 & 9.89 & 420.11 & MM \\
$09-15-94$ & 1455 & Steel tape & 14.05 & 0.01 & 10.38 & 419.62 & MM \\
\hline
\end{tabular}




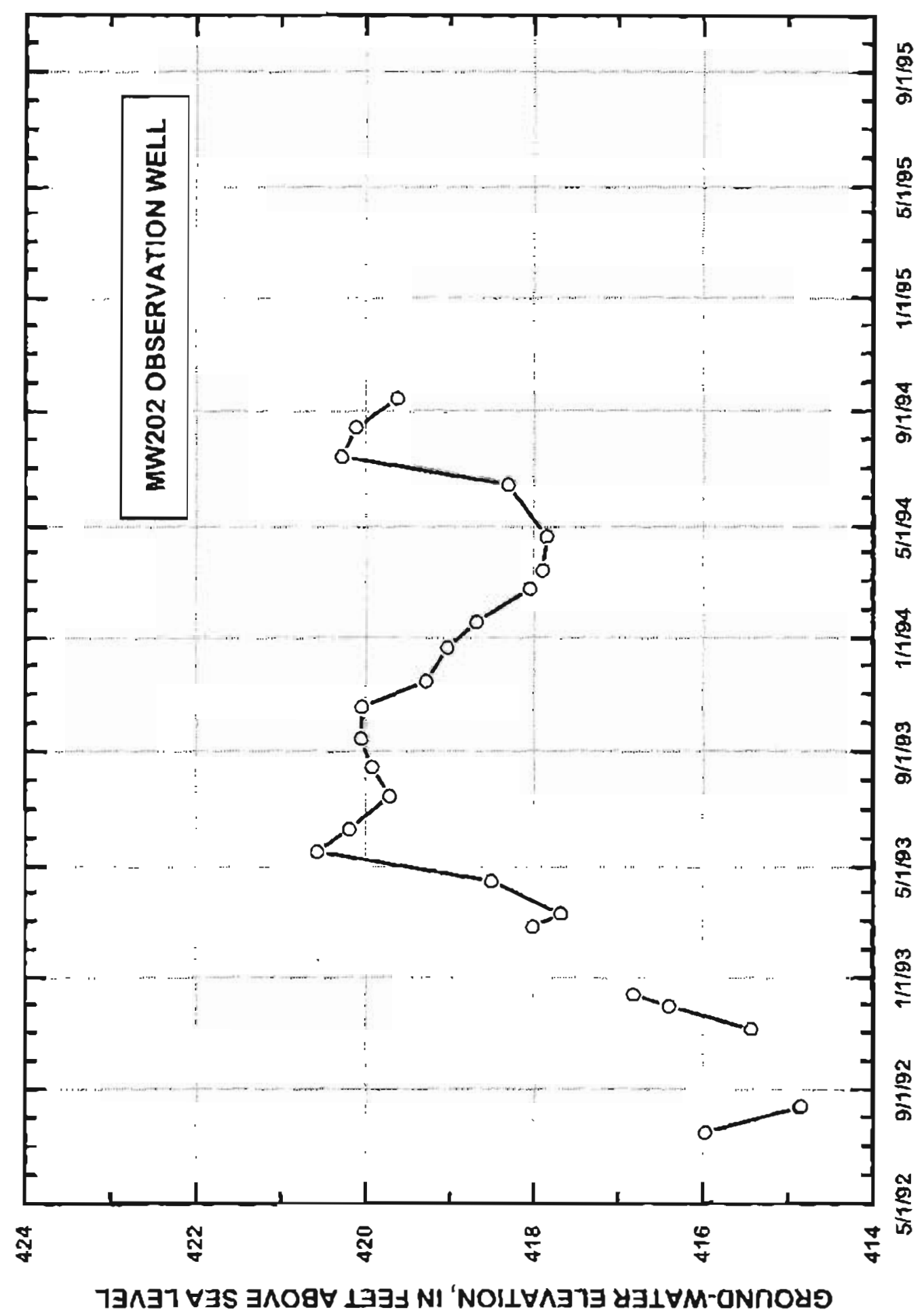


Site ID:

Local Number:

(17147484001

All measurements in feet

Depth to bottom of well from MP

Depth from TOC to top of Sl :

Depth from TOC to bottom of SI :

Land Surface Datum :

\begin{tabular}{c|c} 
Feet & Elevation \\
\hline 19.4 & 414.0 \\
10.9 & 422.6 \\
18.9 & 414.6 \\
& 432.0
\end{tabular}

Datum correclions, reference survey notes in site folders

$$
\begin{array}{c|c}
\text { Date } & \text { MP Elevathon (feet above sea level) } \\
\hline 07-01-92 & 433.46
\end{array}
$$

FC00100106DADB1

LS, land surface

MM, mass measurement

$M P$, measuring point

$N A$, not available

$P M_{1}$ partial measurement

Sl, screened interval

WS, water surface
TOC, top of casing

\begin{tabular}{|c|c|c|c|c|c|c|c|}
\hline Date & Time & Metsod & MP to $\sqrt{\mathrm{NS}}$ & Ertor & LS to WS & WS ejevation & Remarks \\
\hline $07-17-92$ & 1649 & Steel tape & 17.89 & 0.01 & $16 . \overline{43}$ & $4 \longdiv { 1 5 . 5 7 }$ & MMA \\
\hline $08-13-92$ & 2225 & Steel tape & NA & 0.01 & $\mathrm{NA}$ & NA & MM, dry \\
\hline $08-14-92$ & 1230 & E tape & 18.95 & 0.02 & 17.49 & 414.51 & $M M$ \\
\hline $09-14-92$ & 1959 & Steel tape & NA & 0.01 & NA & NA & PM, dry \\
\hline $11-06-92$ & 0940 & Steel tape & 30.03 & 0.01 & 16.57 & 415.43 & MA \\
\hline $12-01-92$ & 1048 & Steel tape & $\uparrow 7.03$ & 0.01 & 15.57 & 416.43 & $M M$ \\
\hline $12-14-92$ & 0911 & Steel tape & 16.63 & 0.01 & 15.17 & $4+6.83$ & MAM \\
\hline $02-20-93$ & 1600 & Steel tape & 15.42 & 0.01 & 13.96 & 418.04 & $\mathrm{MM}$ \\
\hline $02-25-93$ & 1544 & Steel tape & 15.39 & 0.01 & 13.93 & $4+8.07$ & $M M$ \\
\hline $03-12-93$ & 1143 & Steel tape & 15.73 & 0.01 & 14.27 & 417.73 & $M M$ \\
\hline $04-16-93$ & 1356 & Steel tape & 15.04 & 0.01 & 13.58 & 418.42 & $M M$ \\
\hline $05-18-93$ & 0915 & Steel tape & 12.78 & 0.01 & 11.32 & 420.68 & $M N$ \\
\hline $06-11-93$ & 1112 & Steel tape & 1314 & 0.01 & 11.68 & 420.32 & MM \\
\hline $06-30-93$ & 1910 & E-tape & 13.36 & 0.02 & 11.90 & 420.10 & PM \\
\hline $07-16-93$ & 1232 & E-lape & $\uparrow 3.64$ & 0.02 & 12.18 & 419.82 & $M M$ \\
\hline $08-16-93$ & 0830 & Steel tape & 13.45 & 0.01 & 11.99 & 420.01 & $M M$ \\
\hline $09-08-93$ & 1808 & Stecl tape & 13.40 & 0.01 & 11.94 & 420.06 & PM \\
\hline $09-15-93$ & 1613 & E-tape & 13.29 & 0.02 & 11.83 & 420.17 & $M M$ \\
\hline $10-19-93$ & 1234 & Steel tape & 13,28 & 0.01 & 11.82 & 420.18 & MM \\
\hline $11-16-93$ & 1556 & Steel tape & 14.02 & 0.01 & 12.56 & 419.44 & $M M$ \\
\hline
\end{tabular}





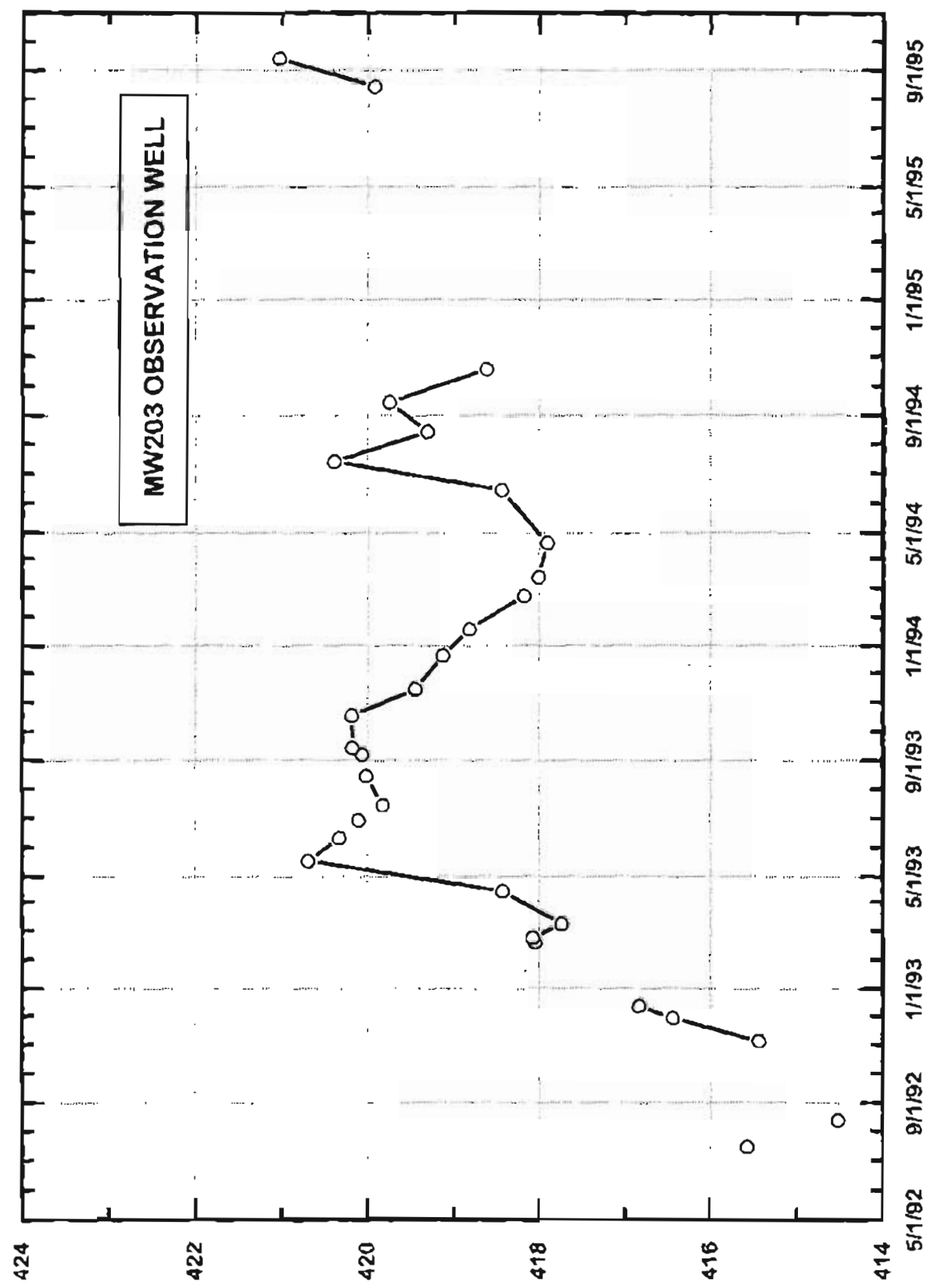

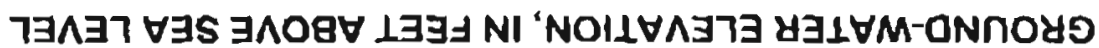


Site iD: Local Number:

\begin{tabular}{c|c} 
Feet & Elevation \\
\hline 18.4 & 413.3 \\
11.9 & 419.8 \\
17.9 & 413.8 \\
& 428.7
\end{tabular}

Datum corrections, reference survey notes in site folders

$$
\begin{array}{c|c}
\text { Date } & \text { MP Elevation (feet above sea level) } \\
\hline 07-29-92 & 431.74
\end{array}
$$

Depth from TOC to top of SI:

Depth from TOC to bottom of $\$ 1$ :

Land Surface Datum :
All measurements in feet

Depth to bottom of well from MP :

\begin{tabular}{cccccccc}
\hline Date & Time & Method & MP to WS & Error & LS to WS & WS elevation & Remarks \\
\hline $07-17-92$ & 1626 & Steel tape & 15.60 & 0.01 & 12.56 & 416.14 & MM \\
$08-13-92$ & 2207 & Steel tape & 16.80 & 0.01 & 13.76 & 414.94 & MM \\
$08-14-92$ & 1255 & Steel tape & 16.82 & 0.01 & 13.78 & 414.92 & PM \\
$09-14-92$ & 1959 & Steel tape & NA & 0.01 & NA & NA & MM, dry \\
$11-06-92$ & 0958 & Steel tape & 16.40 & 0.01 & 13.36 & 415.34 & MM \\
$12-03-92$ & 0950 & Steel tape & 15.36 & 0.01 & 12.32 & 416.38 & MM \\
$12-14-92$ & 0937 & Steel tape & 16.01 & 0.01 & 12.97 & 415.73 & MM \\
$02-12-93$ & 1545 & Steel tape & 13.79 & 0.01 & 10.75 & 417.95 & PM \\
$02-19-93$ & 1119 & Steel tape & 13.79 & 0.01 & 10.75 & 417.95 & MM \\
$02-25-93$ & 1615 & Steel tape & 13.71 & 0.01 & 10.67 & 418.03 & MM \\
$03-12-93$ & 1120 & Steel tape & 14.02 & 0.01 & 10.98 & 417.72 & MM \\
$04-16-93$ & 1413 & Steel tape & 13.25 & 0.01 & 10.21 & 418.49 & MM \\
$05-18-93$ & 0930 & Steel tape & 11.03 & 0.01 & 7.99 & 420.71 & MM \\
$06-11-93$ & 1130 & Steel tape & 10.65 & 0.01 & 7.61 & 421.09 & MM \\
$07-16-93$ & 1224 & Steel tape & 11.96 & 0.01 & 8.92 & 419.78 & MM \\
$08-16-93$ & 0845 & Steel tape & 11.75 & 0.01 & 8.71 & 419.99 & MM \\
$09-08-93$ & 1812 & Steel tape & 11.77 & 0.01 & 8.73 & 419.97 & PM \\
$09-15-93$ & 1558 & E-tape & 11.61 & 0.02 & 8.57 & 420.13 & MM \\
$10-19-93$ & 1240 & Steel tape & 11.57 & 0.01 & 8.53 & 420.17 & MM \\
$11-16-93$ & 1509 & Steel tape & 12.55 & 0.01 & 9.51 & 419.19 & MM
\end{tabular}

\section{LS, land surface} MM, mass measurement MP, measuring point NA, not available $P M_{1}$ partial measurement SI, screened interval TOC, top of casing WS, water surface 
MW204 GROUND-WATER OBSERVATION WELL (ContinuEd)

Site ID:

$64511914748500 \uparrow$

Local Number:

FC00100106DABD1

\begin{tabular}{cccccccc}
\hline Date & Time & Method & MP to WS & Error & LS to WS & WS elevation & Remarks \\
\hline $12-22-93$ & 1140 & E-tape & $\{2.56$ & 0.02 & 9.62 & 419.08 & MM \\
$01-19-94$ & 1231 & Steel tape & 12.99 & 0.01 & 9.95 & 418.75 & MM \\
$03-16-94$ & 1154 & E-tape & 13.79 & 0.02 & 10.75 & 417.95 & MM \\
$04-21-94$ & 1320 & Steel tape & 13.88 & 0.01 & 10.84 & 417.86 & MM \\
$06-15-94$ & 0840 & Steet tape & 13.37 & 0.01 & 10.33 & 418.37 & MM \\
$07-15-94$ & 0959 & Steel tape & 11.46 & 0.01 & 8.42 & 420.28 & MM \\
$08-15-94$ & 1238 & Steel tape & 11.53 & 0.01 & 8.49 & 420.21 & MM \\
$09-15-94$ & 1306 & Steel tape & 12.00 & 0.01 & 8.96 & 419.74 & MM \\
$10-18-94$ & 1538 & Steel tape & 13.14 & 0.01 & 10.10 & 418.60 & MM \\
$10-20-94$ & 1330 & Steel tape & 13.15 & 0.01 & 10.11 & 418.59 & MM \\
$04-15-95$ & 1059 & Steel tape & 14.07 & 0.01 & 11.03 & 417.67 & MM \\
$06-15-95$ & 1437 & E-tape & 12.85 & 0.02 & 9.81 & 418.89 & MM \\
\hline
\end{tabular}




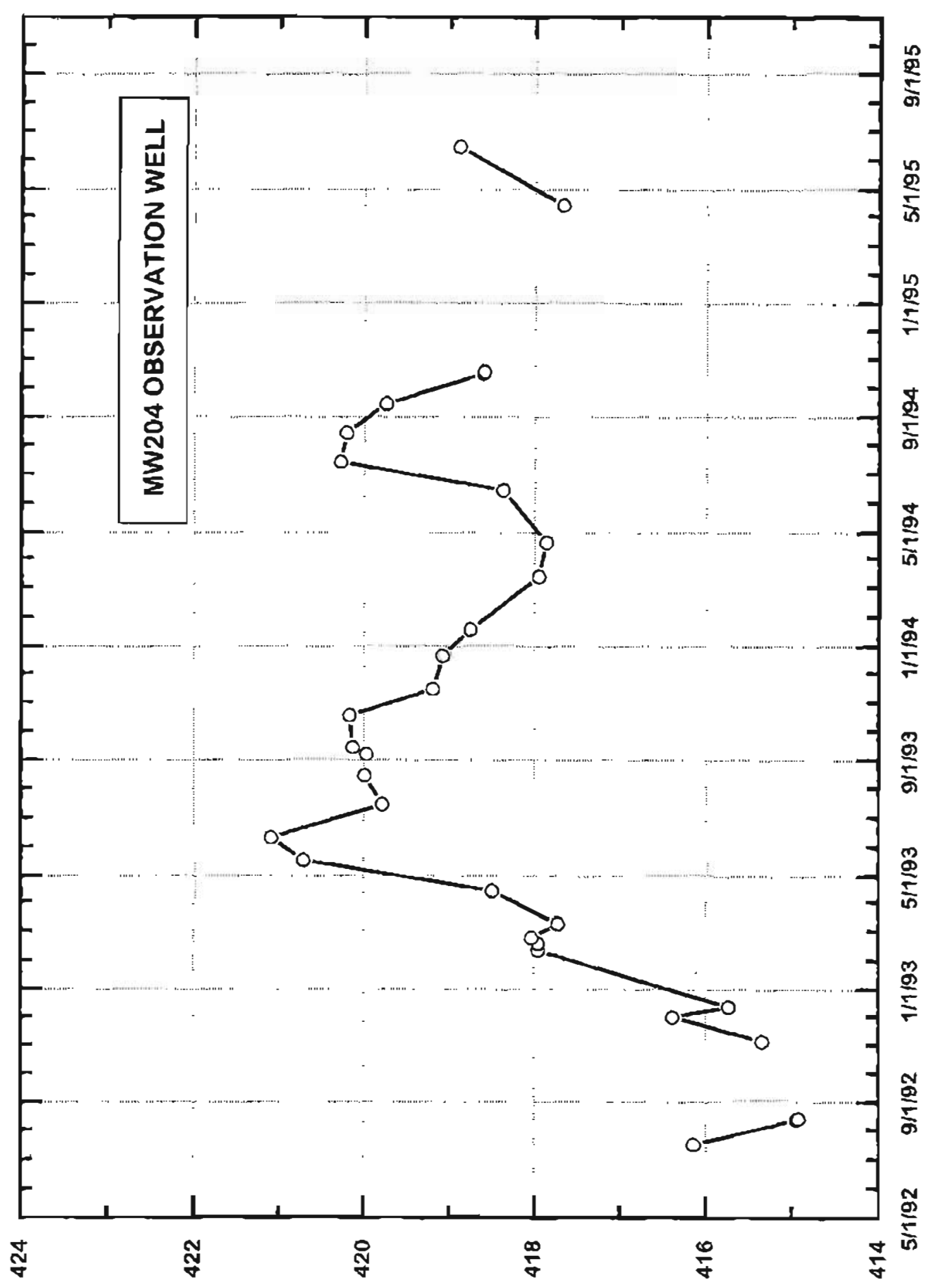

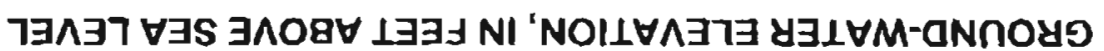


Site ID:

Local Number:

\begin{tabular}{c|c} 
Feet & Elevation \\
\hline 19.5 & 412.9 \\
9.0 & 423.4 \\
19.0 & 413.4 \\
& 430.9
\end{tabular}

All measurements in feet

Depth to bottom of well from MP:

Depth from TOC to top of SI:

Depth from TOC to bottom of $S i$ :

Land Surface Datum :

Datum corrections, reference survey notes in site folders

$$
\begin{array}{c|c}
\text { Date } & \text { MP Elevation (feet above sea lovel) } \\
\hline 07-01-92 & 432.38
\end{array}
$$

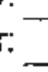
645115147485701 FC00100106DBDA1

LS, land surface MM, mass measurement $M P$, measuring point NA, not available PM, partial measurement SI, screened interval TOC, top of casing

\begin{tabular}{|c|c|c|c|c|c|c|c|}
\hline Date & Time & Method & $\overline{M P}$ to WS & Error & LS to WS & WS elevation & Remarks \\
\hline $07-17-92$ & 1517 & Steel tape & 15.86 & 0.01 & 14.38 & 416.52 & NA \\
\hline $08-13-92$ & 2124 & Steel tape & 16.89 & 0.01 & 15.41 & 415.49 & MM \\
\hline $08-14-92$ & 1318 & Steel tape & 16.92 & 0.01 & 15.44 & 415.46 & PM \\
\hline $09-14-92$ & 1808 & Steel tape & NA & 0.01 & NA & NA & $P M, d r y$ \\
\hline $11-06-92$ & 1640 & Steel tape & 17.76 & 0.01 & 16.28 & 414.62 & MM \\
\hline $12-01-92$ & 1142 & Steel tape & 16.00 & 0.01 & 14.52 & 416.38 & MM \\
\hline $12-14-92$ & 1024 & Steel tape & 15.54 & 0.01 & 14.06 & 416,84 & MM \\
\hline $02-19-93$ & 1217 & Steel tape & 15.30 & 0.01 & 13.82 & 417.08 & $M M$ \\
\hline $02-25-93$ & 1635 & Steel tape & 15.35 & 0.01 & 13.87 & 417.03 & $\mathrm{MM}$ \\
\hline $03-12-93$ & 1050 & Steel tape & 14.76 & 0.01 & 13.28 & 417.62 & $M M$ \\
\hline $04-16-93$ & 1447 & Steel tape & 13.54 & 0.01 & 12.06 & 418.84 & MM \\
\hline $05-18-93$ & 1010 & Steel tape & 12.65 & 0.01 & 11.17 & 419.73 & $M M$ \\
\hline $06-11-93$ & 1122 & Steel tape & 12.23 & 0.01 & 10.75 & 420.15 & MM \\
\hline $06-30-93$ & 1919 & E-tape & 12.49 & 0.02 & 11.01 & 419.89 & $P M$ \\
\hline $07-16-93$ & 1213 & Steel tape & 12.73 & 0.01 & 11.25 & 419.65 & $M M$ \\
\hline $08-16-93$ & $0 ९ 08$ & Steel tape & 12.51 & 0.01 & 11.03 & 419.87 & MN \\
\hline $09-15-93$ & 1629 & E-tape & 13.17 & 0.02 & 11.69 & 419.21 & MM \\
\hline $10-19-93$ & 1256 & Steel tape & 12.39 & 0.01 & 10.91 & 419.99 & $M M$ \\
\hline $11-16-93$ & 1612 & Steel tape & 13.17 & 0.01 & 11.69 & 419.21 & $M A A$ \\
\hline $12-22-93$ & 1108 & E-tape & 13.46 & 0.02 & 11.98 & 418.92 & $M M$ \\
\hline
\end{tabular}
WS, water surface 
Site ID: Local Number:

645115147485701

FC00100106DBDA1

\begin{tabular}{cccccccc}
\hline Dale & Time & Method & MP to WS & Error & LS to WS & WS elevation & Remarks \\
\hline $01-19-94$ & 1222 & Steel tape & 13.81 & 0.01 & 12.33 & 418.57 & MM \\
$02-24-94$ & 1315 & Steel tape & 14.42 & 0.01 & $\{2.94$ & 417.96 & MM \\
$03-16-94$ & 1214 & E-tape & 14.55 & 0.02 & 13.07 & 417.83 & MM \\
$04-21-94$ & 1436 & Steel tape & 14.46 & 0.01 & 12.98 & 417.92 & MM \\
$06-15-94$ & 0913 & Steel tape & 14.13 & 0.01 & 12.65 & 418.25 & MM \\
$07-15-94$ & 1017 & Steel tape & 12.20 & 0.01 & 10.72 & 420.18 & MM \\
$08-15-94$ & 1219 & Steel tape & 12.24 & 0.01 & 10.76 & 420.14 & MM \\
$09-15-94$ & 1253 & Steel tape & 12.78 & 0.01 & 11.30 & 419.60 & AM \\
\hline
\end{tabular}




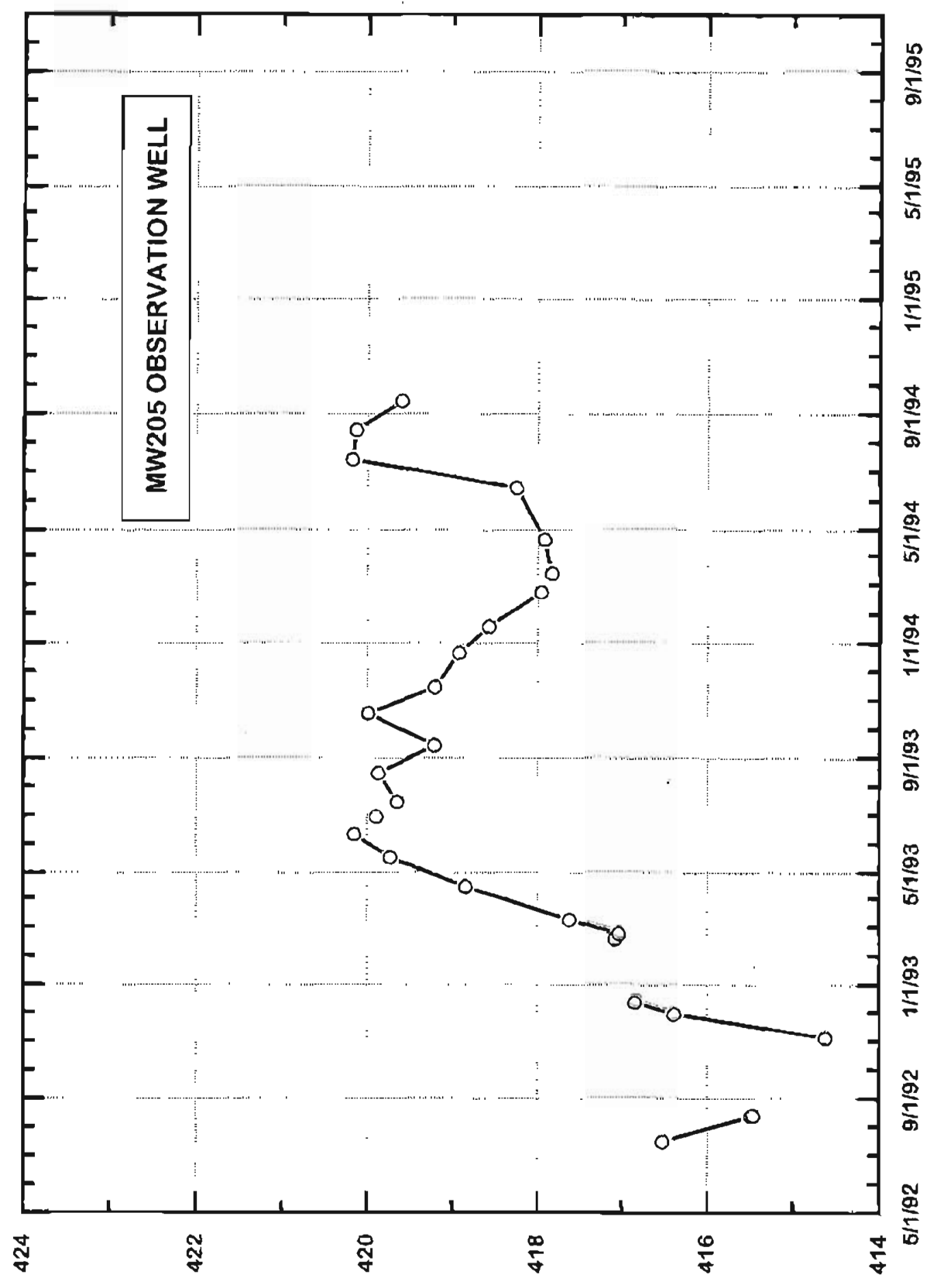

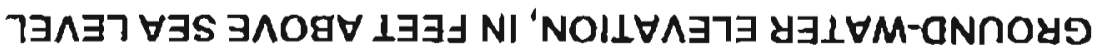




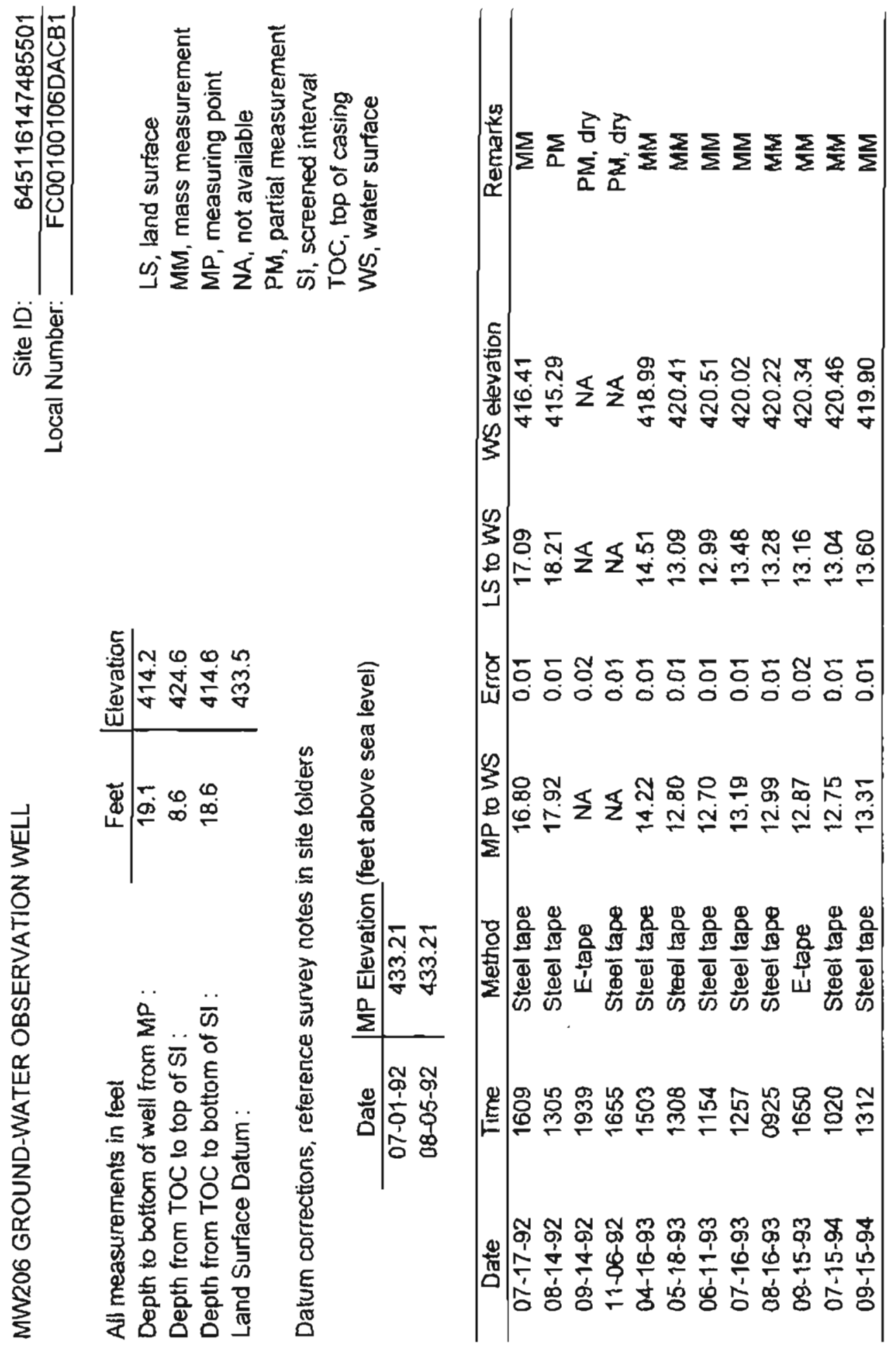




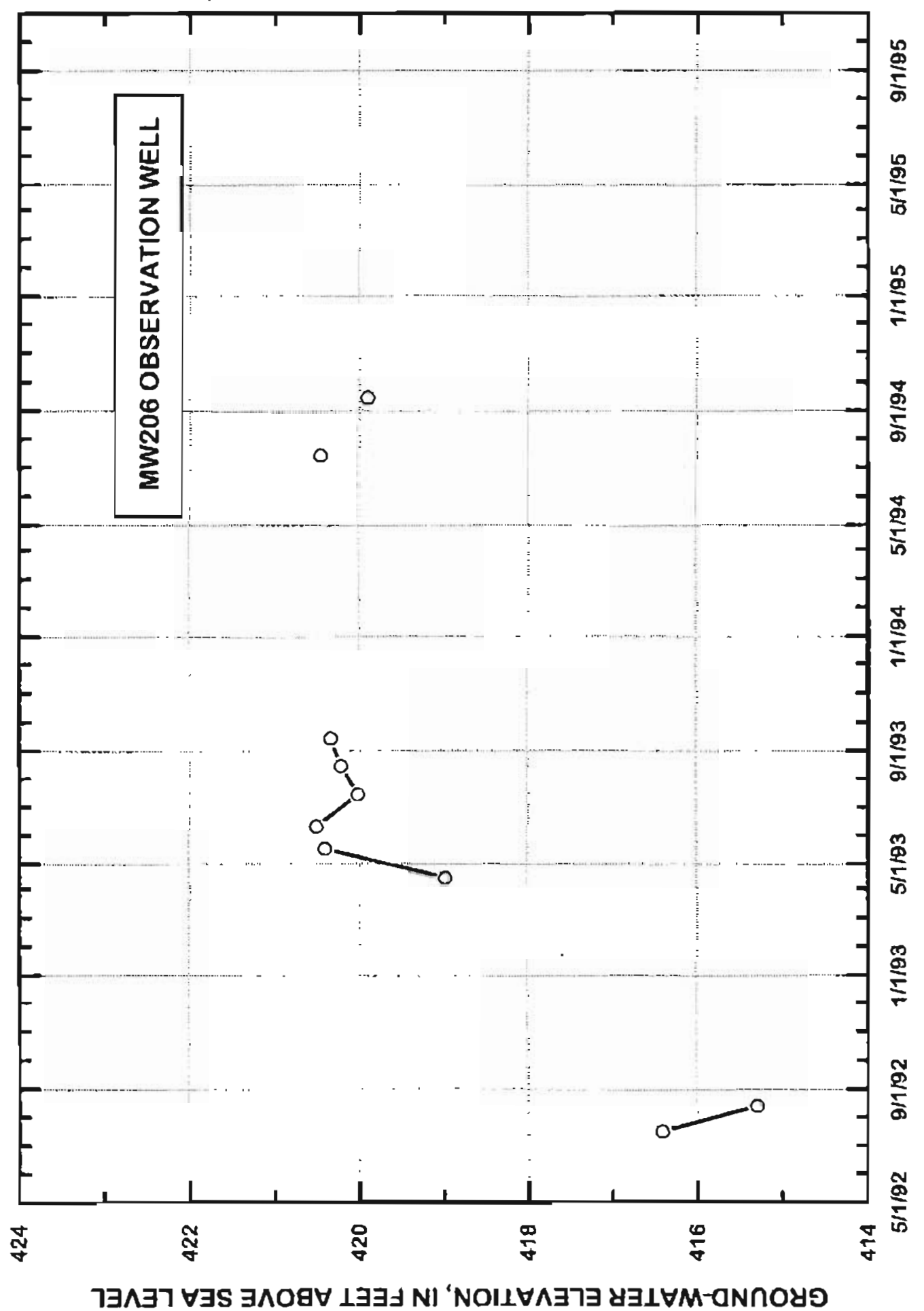


MW207 GROUND-WATER OBSERVATION WELL

CONTINUOUS-DATA COLLECTION STATION, initially called GW 1

All measurements in feet

Depth to bottom of well from MP :

Depth from TOC to top of SI :

Depth from TOC to bottom of SI:

Land Surface Datum :

\begin{tabular}{c|c} 
Feet & Elevation \\
\hline $29 . \overline{8}$ & $\overline{403.6}$ \\
16.8 & 416.6 \\
26.8 & 406.6 \\
& 430.7
\end{tabular}

Datum corrections, reference survey notes in site folders

\begin{tabular}{c|cc} 
Date & MP elevation (feet above sea level) \\
\hline $08-05-92$ & 433.36 & \\
$02-13-93$ & 433.38 & new MP \\
$08-18-93$ & 433.33 & updated MP
\end{tabular}

Site ID:

Local Number:

645059147484101 FC00100106CDDC1

LS, land surface $M M$, mass measurement $M P$, measuring point NA, not available PM, partial measurement SI, screened interval TOC, top of casing WS, water surface

\begin{tabular}{|c|c|c|c|c|c|c|c|}
\hline Date & Time & Method & MP to WS & Error & LS to WS & WS elevation & Remarks \\
\hline $06-17-92$ & 1541 & Steel tape & 14.36 & 0.01 & 11.73 & 419.00 & $\mathrm{PM}$ \\
\hline $07-17-92$ & 1330 & Steel tape & 15.65 & 0.01 & 13.02 & 417.71 & MM \\
\hline $08-13-82$ & 2104 & Steel tape & 15.95 & 0.01 & 13.32 & 417.41 & MM \\
\hline $08-26-92$ & 1125 & Steel tape & 16.65 & 0.01 & 14.02 & 416.71 & PM \\
\hline $09-13-92$ & 2021 & Steel tape & 18.14 & 0.01 & 15.51 & 415.22 & PM \\
\hline $09-14-92$ & 1715 & Steel tape & 18.23 & 0.01 & 15.60 & 415.13 & PM \\
\hline $11-06-92$ & 1300 & Steel tape & 18.48 & 0.01 & 15.85 & 414.88 & MM \\
\hline $12-01-92$ & 1004 & Steel tape & 16.33 & 0.01 & 13.70 & 417.03 & PM \\
\hline $12-08-92$ & 1045 & Steel tape & 17.25 & 0.01 & 14.62 & 416.11 & PM \\
\hline $12-14-92$ & 1627 & Steel tape & 17.18 & 0.01 & 14.55 & 416.18 & MM \\
\hline $02-01-93$ & 1004 & Steel tape & 16.33 & 0.01 & 13,70 & 417.03 & $P M$ \\
\hline $02-10-93$ & 1355 & Steel tape & 16.43 & 0.01 & 13.80 & 416.93 & PM \\
\hline $02-13-93$ & 1321 & Steel tape & 16.52 & 0.01 & 13.89 & 416.84 & Installed instr. shelter \\
\hline $02-19-93$ & 0919 & Steel tape & 16.50 & 0.01 & 13.87 & 416.88 & MM \\
\hline $02-22-93$ & 1532 & Steel tape & 16.61 & 0.01 & 13.98 & 416.77 & $\mathrm{PM}$ \\
\hline $02-24-93$ & 1357 & Steel tape & 16.62 & 0.01 & 13.99 & 416.76 & MM \\
\hline $02-25-93$ & 1127 & Steel tape & 16.58 & 0.01 & 13.95 & 416.80 & MM \\
\hline $03-12-93$ & 1046 & Steel tape & 16.64 & 0.01 & 14.01 & 416.74 & MM \\
\hline
\end{tabular}


MW207 GROUND-WATER OBSERVATION WELL (Continued) CONTINUOUS-DATA COLLECTION STATION, initially called GW- 1

Site ID: Local Number:

645059147484101

\begin{tabular}{|c|c|c|c|c|c|c|c|}
\hline Date & Time & Method & MP to WS & Error & LS to WS & WS elevation & Remarks \\
\hline $03-17-93$ & 1539 & Steel tape & 16.87 & 0.01 & 14.24 & $4 \overline{16.51}$ & $\mathrm{PM}$ \\
\hline $0402-93$ & 1643 & Steel tape & 17.07 & 0.01 & 14.44 & 416.31 & PM \\
\hline $04-09-93$ & 1142 & Steel lape & 17.02 & 0.01 & 14.39 & 416.36 & PM \\
\hline $04-\{2-93$ & 1642 & Steel tape & 16.77 & 0.01 & 14.14 & 416.61 & $\mathrm{PM}$ \\
\hline $04-14-93$ & 1330 & Steel tape & 16.60 & 0.01 & 13.97 & 416.78 & PH \\
\hline $04-16-93$ & 0907 & Steel tape & 16.45 & 0.01 & 13.82 & 416.93 & MM \\
\hline $04-19-93$ & 1725 & Steel tape & 16.21 & 0.01 & 13.58 & $4 \uparrow 7.17$ & PM \\
\hline $04-21-93$ & 1604 & Steel tape & 16.01 & 0.01 & 13.38 & 417.37 & PM \\
\hline $04-23-93$ & 1604 & Steel lape & 15.78 & 0.01 & 13.15 & 417.60 & PM \\
\hline $05-02-93$ & 1755 & Steel tape & 14.85 & 0.01 & 12.22 & 418.53 & PM \\
\hline $05-03-93$ & 1055 & Steel tape & 14.78 & 0.01 & 12.15 & 418.60 & PM \\
\hline $05-05-93$ & 1149 & Steel lape & 14.57 & 0.01 & 11.94 & 418.81 & PM \\
\hline $05-08-93$ & 2106 & Steel tape & 14.35 & 0.01 & 11.72 & 419.03 & PM \\
\hline $05-10-93$ & 1340 & Steel kape & 14.17 & 0.01 & 11.54 & 419.21 & PM \\
\hline $05-12-93$ & 1103 & Steel tape & $\$ 4.08$ & 0.01 & 11.45 & $\$ 19.30$ & PM \\
\hline $05-\{4-93$ & 1010 & Steel tape & 13.96 & 0.01 & 11.33 & 419.42 & PM \\
\hline $05-18-93$ & 1430 & Steel tape & 13.82 & 0.01 & 11.19 & 419.51 & MM \\
\hline $05-24-93$ & 1346 & Steel tape & 13.72 & 0.01 & 11.09 & 419.61 & PM \\
\hline $05-26-93$ & 1706 & Steel tape & 14.02 & 0.01 & 11.39 & 419.31 & $\mathrm{PM}$ \\
\hline $05-29-93$ & 1345 & Steel tape & 14.02 & 0.01 & 11.39 & 419.31 & PM \\
\hline $06-02-93$ & 1532 & Steel tape & 14.26 & 0.01 & 11.63 & 419.07 & PM \\
\hline $06-08-93$ & 1348 & Steet tape & 14.53 & 0.01 & 11.90 & 418.80 & $P M$ \\
\hline $06-11-93$ & 1024 & Steel tape & 14.61 & 0.01 & 11.98 & 418.72 & MM \\
\hline $06-21-93$ & 1025 & Steel tape & 14.72 & 0.01 & 12.09 & 418.61 & $\mathrm{PM}$ \\
\hline $07-09-93$ & 0854 & Steel tape & 14.74 & 0.01 & 12.11 & 418.59 & $\mathrm{PM}$ \\
\hline $07-16-93$ & 1124 & Steel tape & 15.14 & 0.01 & 12.51 & 418.19 & $M M$ \\
\hline $07-21-93$ & 1438 & Steel tape & 14.83 & 0.01 & 12.20 & 418.50 & PM \\
\hline $07-28-93$ & 1738 & Steel tape & 14.6 & 0.01 & 11.97 & $4 \uparrow 8.73$ & PM \\
\hline $08-05-93$ & 1825 & Steel tape & 14.53 & 0.01 & 11.90 & 418.80 & PM \\
\hline $08-11-93$ & 1410 & Steel tape & 14.33 & 0.01 & 11.70 & 419.00 & PM \\
\hline $08-16-93$ & 1041 & Steel tape & 14.42 & 0.01 & 11.79 & 418.91 & $M M$ \\
\hline
\end{tabular}




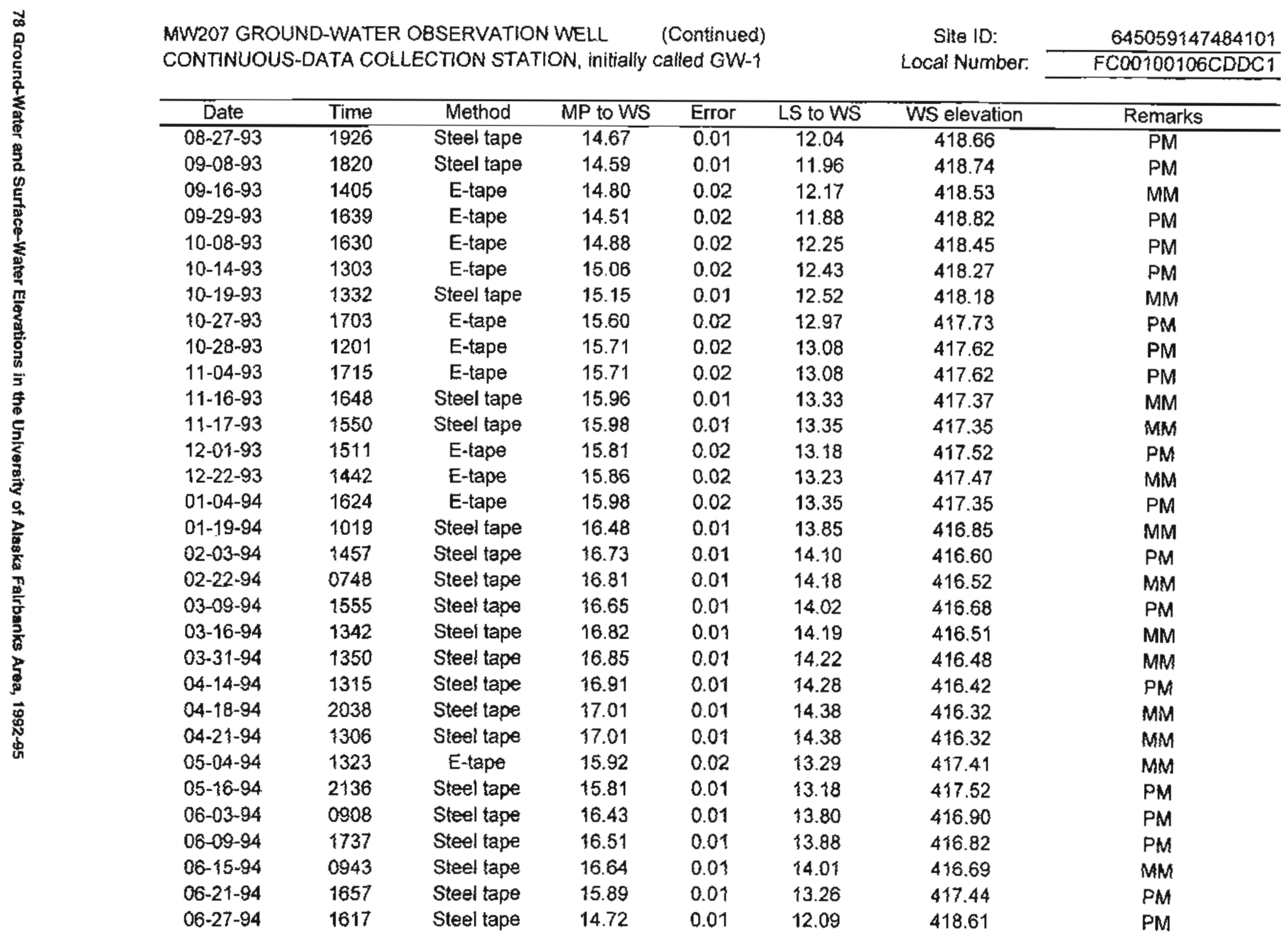


MW207 GROUND-WATER OBSERVATION WELL (COntinued)

Site ID:

645059147484101 CONTINUOUS-DATA COLLECTION STATION, initally called GW-1

Local Number.

FC00100106CDDC1

\begin{tabular}{|c|c|c|c|c|c|c|c|}
\hline Date & Time & Method & MP to WS & Error & LS to WS & WS elevation & Remarks \\
\hline $07-01-94$ & 1528 & Steel tape & 14.03 & 0.01 & 11.40 & 419.30 & PM \\
\hline $07-15-94$ & 0955 & Steel tape & 14.34 & 0.01 & 11.71 & 418.99 & MM \\
\hline $07-21-94$ & 1045 & Steel tape & 14.35 & 0.01 & 11.72 & 418.98 & $\mathrm{PM}$ \\
\hline 08-03-94 & 1432 & Steel tape & 14.47 & 0.01 & 11.84 & 418.86 & $\mathrm{PM}$ \\
\hline $08-12-94$ & 1550 & Steel tape & 14.07 & 0.01 & 11.44 & 419.26 & PM \\
\hline $08-15-94$ & 1007 & Steel tape & 13.93 & 0.01 & 11.30 & 419.40 & MM \\
\hline $09-08-94$ & 1747 & Steel tape & 14.87 & 0.01 & 12.24 & 418.46 & PM \\
\hline $09-15-94$ & 1430 & Steel tape & 15.25 & 0.01 & 12.62 & 418.08 & MNA \\
\hline $09-29-94$ & 1334 & Steel tape & 16.11 & 0.01 & 13.48 & 417.22 & PM \\
\hline $10-25-94$ & 1329 & Steel tape & 16.95 & 0.01 & 14.32 & 416.38 & PM \\
\hline $11-16-94$ & 1545 & Steel tape & 17.03 & 0.01 & 14.40 & 416.30 & MM \\
\hline $12-02-94$ & 1430 & E-tape & 17.02 & 0.02 & 14.39 & 416.31 & PM \\
\hline $12-23-94$ & 1517 & Steel tape & 16.70 & 0.01 & 14.07 & 416.63 & MM \\
\hline $01-18.95$ & 1523 & Steel tape & 17.10 & 0.01 & 14.47 & 416.23 & AM \\
\hline $02-16-95$ & 1546 & Steel tape & 17.36 & 0.01 & 14.73 & 415.97 & MM \\
\hline $02-21-95$ & 1658 & Steel tape & NA & 0.01 & $N A$ & NA & PA \\
\hline $02-22-95$ & 1620 & Steel tape & 17.29 & 0.01 & 14.66 & 416.04 & PT \\
\hline $02-27-95$ & 0830 & Steel tape & 17.06 & 0.01 & 14.43 & 416.27 & PT \\
\hline $03-14-95$ & 1545 & Steat tape & 17.35 & 0.01 & 14.72 & 415.98 & PT \\
\hline $04-15-95$ & 1204 & Steel tape & 17.24 & 0.01 & 14.61 & 416.09 & MM \\
\hline $05-16-95$ & 1430 & Steel tape & 15.38 & 0.01 & 12.75 & 417.95 & $M M$ \\
\hline $05-26-95$ & 1125 & Steel tape & $\{5.52$ & 0.01 & 12.89 & 417.81 & PM \\
\hline $07-17-95$ & 1415 & Steel tape & 14.07 & 0.01 & 11.44 & 419.26 & MM \\
\hline $08-16-95$ & 1607 & Steel tape & 14.55 & 0.01 & 11.92 & 418.78 & MM \\
\hline $09-14-95$ & 1633 & E-tape & 13.51 & 0.02 & 10.88 & 419.82 & MM \\
\hline $10-05-95$ & 1520 & Steel tape & 14.27 & 0.01 & 11.64 & 419.06 & PM \\
\hline $10-17-95$ & 1229 & E-tape & 14.91 & 0.02 & 12.28 & 418.42 & MM \\
\hline
\end{tabular}




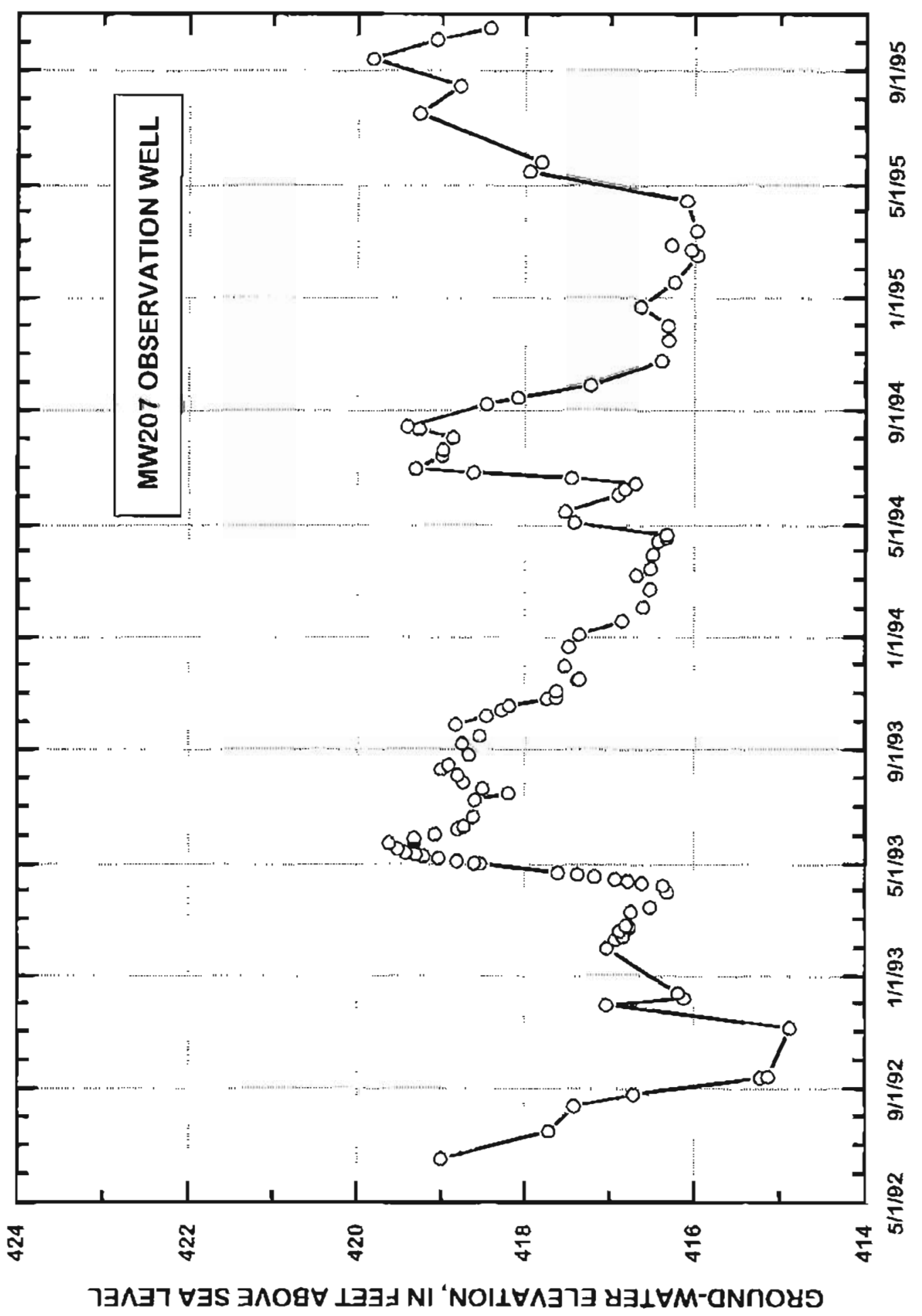


MW208 GROUND-WATER OESERVATION WELL

Initially called GW2

All measurements in feet

Depth to bottom of well from MP :

Depth from TOC to top of Si:

Depth trom TOC to bottom of SI :

Land Surface Datum:

\begin{tabular}{c|c} 
Feet & Elevation \\
\hline 22.3 & 410.4 \\
10.8 & 421.8 \\
20.80 & 411.81 \\
& 430.7
\end{tabular}

Datum corrections, reference survey notes in site folders \begin{tabular}{c|c} 
Date & MP Elevation (feel above sea level) \\
\hline $07-01-92$ & 432.61
\end{tabular}
Site lD:

Local Number

645059147494901 FC00100106CDCD1

LS, land surface

$M M$, mass measurement

MP: measuring point

$\mathrm{PM}$, partial measurement

SI, screened interval

TOC, top of casing

WS, water surface

\begin{tabular}{|c|c|c|c|c|c|c|c|}
\hline Date & Time & Method & MP to WS & Error & LS to WS & WS elevation & Remarks \\
\hline $06-18-92$ & 0800 & E-tape & 13.39 & 0.02 & 11.48 & $4 \overline{419.22}$ & $P M$ \\
\hline $07-17-92$ & 1254 & Steel tape & 14.61 & 0.01 & 12.70 & 418.00 & MM \\
\hline $08-13-92$ & 2030 & Steel tape & 14.92 & 0.01 & 13.01 & 417.69 & MM \\
\hline $08-26-92$ & 1200 & Steel tape & 15.53 & 0.01 & 13.62 & 417.08 & PM \\
\hline $09-14-92$ & 1735 & Steel tape & 16.90 & 0.01 & 14.99 & 415.71 & PM \\
\hline $11-06-92$ & 1420 & Steel tape & 17.67 & 0.01 & 15.66 & 415.04 & MM \\
\hline $12-08-92$ & 1017 & Steel tape & 16.53 & 0.01 & 14.62 & 416.08 & PM \\
\hline $12-14-92$ & 1610 & Steel tape & 16.45 & 0.01 & 14.54 & 416.16 & MM \\
\hline $02-25-93$ & 1221 & Steel tape & 15.82 & 0.01 & 13.91 & 416.79 & MM \\
\hline $03-12-93$ & 1109 & Steel tape & 15.68 & 0.01 & 13.97 & 416.73 & $\mathrm{MM}$ \\
\hline $04-16-93$ & 1550 & Steel tape & 15.69 & 0.01 & 13.78 & 416.92 & MM \\
\hline $05-18-93$ & 1055 & Steel tape & 12.84 & 0.01 & 10.93 & 419.77 & MM \\
\hline $06-11-93$ & 1019 & Steel tape & 13.56 & 0.01 & 11.65 & 419.05 & $M M$ \\
\hline $07-16-93$ & 1138 & Steel tape & 14.06 & 0.01 & 12.15 & 478.55 & $M M$ \\
\hline $08-16-93$ & 0134 & Steel tape & 12.42 & 0.01 & 10.51 & 420.19 & $M M$ \\
\hline $10-19-93$ & 1415 & Steel tape & 13.95 & 0.01 & 12.04 & 418.66 & $M M$ \\
\hline $10-27-93$ & 1740 & E-tape & 14.43 & 0.02 & 12.52 & 418.18 & PM \\
\hline $10-28-93$ & 1152 & Etape & 14.33 & 0.02 & 12.42 & 418.28 & PM \\
\hline $12-22-93$ & 1432 & E-tape & 13.74 & 0.02 & 11.83 & 418.87 & MM \\
\hline $02-24-94$ & 1456 & Steel tape & 15.89 & 0.01 & 13.98 & 416.72 & MM \\
\hline
\end{tabular}


MW208 GROUND-WATER OBSERVATION WELL (ContinUEd) Initially called $\mathrm{GW} 2$

Site 10: Local Number:

645059147494901

\begin{tabular}{cccccccc}
\hline Date & Time & Method & MP to WS & Error & LS to WS & WS elevation & Remarks \\
\hline $04-21-94$ & 1445 & Steel tape & 16.10 & 0.01 & 14.19 & $416.5 t$ & MM \\
$05-16-94$ & 2211 & Steel tape & 14.98 & 0.01 & 13.07 & 417.63 & MM \\
$06-15-94$ & 0848 & Steel tape & 15.75 & 0.01 & 13.84 & 416.86 & MM \\
$07-15-94$ & 1040 & Steel tape & 13.52 & 0.01 & 11.61 & 419.09 & MM \\
$08-15-94$ & 1017 & Steel tape & 13.16 & 0.01 & 11.25 & 419.45 & MM \\
$09-15-94$ & 1341 & Steel tape & 14.39 & 0.01 & 12.28 & 418.42 & MM \\
$10-20-94$ & 1239 & Steel tape & 15.76 & 0.01 & 13.85 & 416.85 & MM \\
\hline
\end{tabular}




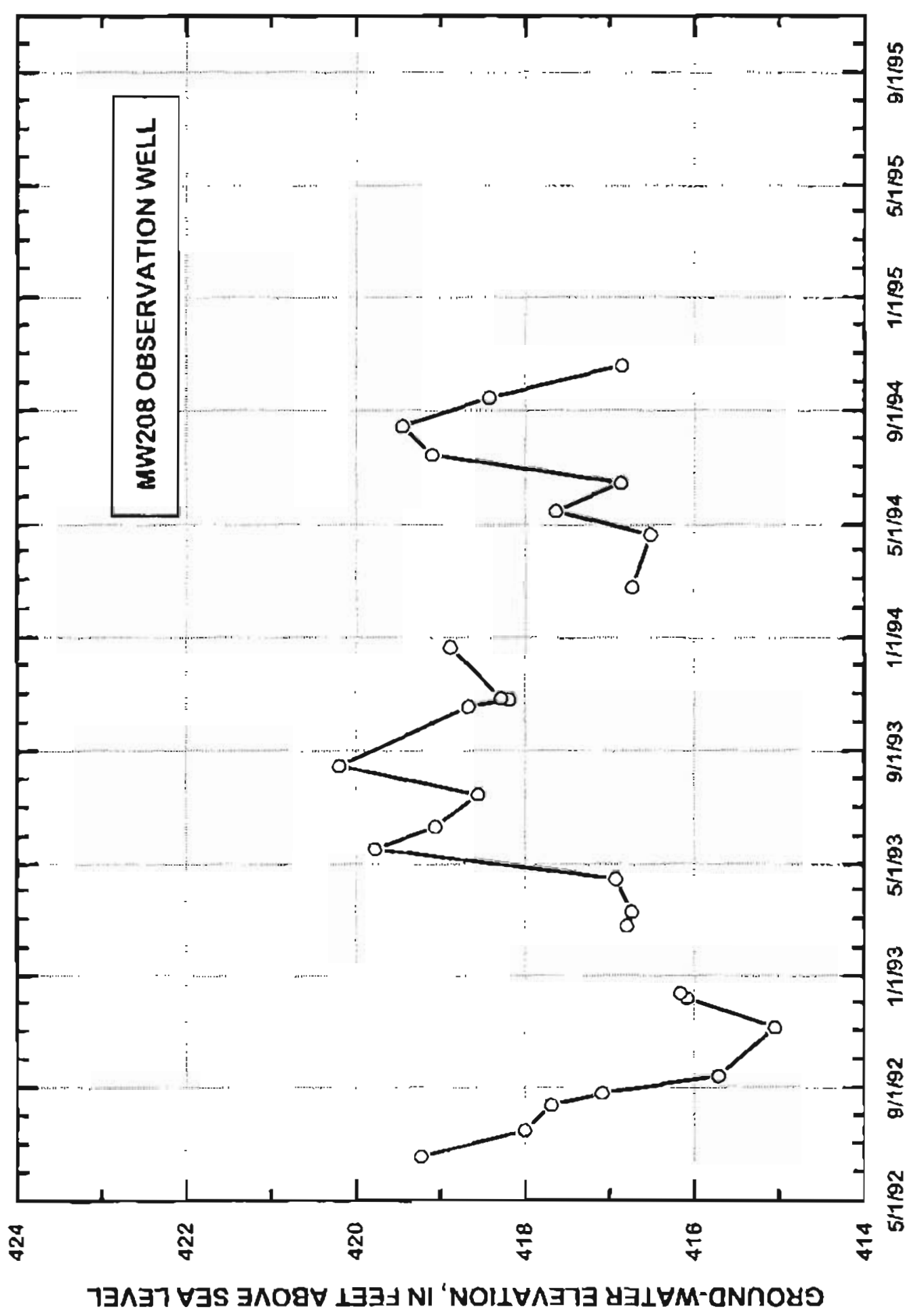


Site ID: Local Number. 645059147493401

\begin{tabular}{lc|c}
\cline { 2 - 3 } Depth to bottom of well from MP : & 21.3 & 410.0 \\
Depth from TOC to top of SI : & 11.3 & 420.0 \\
Depth from TOC to bottom of SI : & 21.3 & 410.0 \\
Land Surface Datum : & & 430.9
\end{tabular}

Datum corrections, reference survey notes in site folders

\begin{tabular}{|c|c|c|c|c|c|c|c|}
\hline \multicolumn{3}{|c|}{ Depth to bottom of well from MP : } & 21.3 & 410.0 & & & LS land suface \\
\hline \multirow{3}{*}{\multicolumn{3}{|c|}{$\begin{array}{l}\text { Depth from TOC lo top of SI: } \\
\text { Depth from TOC to bottom of SI: } \\
\text { Land Surface Datum: }\end{array}$}} & 11.3 & 420.0 & & & \multirow{8}{*}{$\begin{array}{l}\text { MM, mass measurement } \\
\text { MP, measuring point } \\
\text { NA, not avallable } \\
\text { PM, partial measurement } \\
\text { S1, screened interval } \\
\text { TOC, top of casing } \\
\text { WS, water surface }\end{array}$} \\
\hline & & & 21.3 & 410.0 & & & \\
\hline & & & & 430.9 & & & \\
\hline & & & & & & & \\
\hline \multirow{2}{*}{\multicolumn{7}{|c|}{ Datum corrections, reference survey notes in site folders }} & \\
\hline & & & & & & & \\
\hline & Date & MP Elevation & et above se & levet) & & & \\
\hline & $10-31-93$ & $4 \overline{31.28}$ & & & & & \\
\hline Date & Time & Method & MP to WS & Error & LS to WS & WS elevation & Remarks \\
\hline $11-17-92$ & NÄ & NA & 19.9 & 0.02 & 19.52 & 411.38 & Gilfilian (1993) \\
\hline $12-04-92$ & 1310 & Steel tape & NA & 0.01 & NA & NA & MM, dry/frozen \\
\hline $12-14-92$ & 1156 & Steel tape & NA & 0.01 & NA & NA & MM, dryifrozen \\
\hline $02-22-93$ & 1656 & Steel tape & NA & 0.01 & NA & NA & PM, dry/frozen \\
\hline $04-16-93$ & 1558 & Steed tape & NA & 0.01 & NA & NA & MM, dryffrozen \\
\hline $05-18-93$ & 1535 & Steel tape & NA & 0.01 & NA & NA & $M M$, dry/frozen \\
\hline $06-11-93$ & 1025 & Steel tape & NA & 0.01 & NA & NA & MM, dry \\
\hline $07-17-93$ & 1323 & Steel tape & 15.89 & 0.01 & 15.51 & 415.39 & MM \\
\hline $08-16-93$ & 1152 & Steel tape & 14.14 & 0.01 & 13.76 & 417.14 & MM \\
\hline $09-15-93$ & 1645 & Steel tape & 12.08 & 0.01 & 11.70 & 419.20 & MM \\
\hline $10-19-93$ & 1405 & Steel tape & 12.49 & 0.01 & 12.11 & 418.79 & $M M$ \\
\hline $10-27-93$ & 1809 & E-tape & 12.70 & 0.02 & 12.32 & 418.58 & $M M$ \\
\hline $10-28-93$ & 1136 & E-tape & 12.65 & 0.02 & 12.27 & 418.63 & MM \\
\hline $11-16-93$ & 1400 & E-tape & 12.65 & 0.02 & 12.27 & 418.63 & MM \\
\hline $12-22-93$ & 1415 & E-tape & 13.29 & 0.02 & 12.91 & 417.99 & MM \\
\hline $04-21-94$ & 1405 & Steel tape & 13.54 & 0.01 & 13.16 & 417.74 & MM \\
\hline $06-15-94$ & 0923 & Sted tape & 12.54 & 0.01 & 12.16 & 418.74 & MM \\
\hline $07-15-94$ & 1055 & Steel tape & 11.86 & 0.01 & 11.48 & 419.42 & MM \\
\hline $08-15-94$ & 1356 & Steel tape & 14.36 & 0.01 & 13.98 & 416.92 & MM \\
\hline $09-15-94$ & 1400 & Steel tape & 12.58 & 0.01 & 12.20 & 418.70 & MM \\
\hline
\end{tabular}


MW209 GROUND-WATER OBSERVATION WELL (COntinued)

Site ID:

645059147493401

Local Number: FC00100106CDDD1

\begin{tabular}{cccccccc}
\hline Date & Time & Method & MP to WS & Error & LS to WS & WS elevation & Remarks \\
\hline $10-20-94$ & 1253 & Steel tape & 13.30 & 0.01 & 12.92 & 417.98 & MM \\
\hline
\end{tabular}




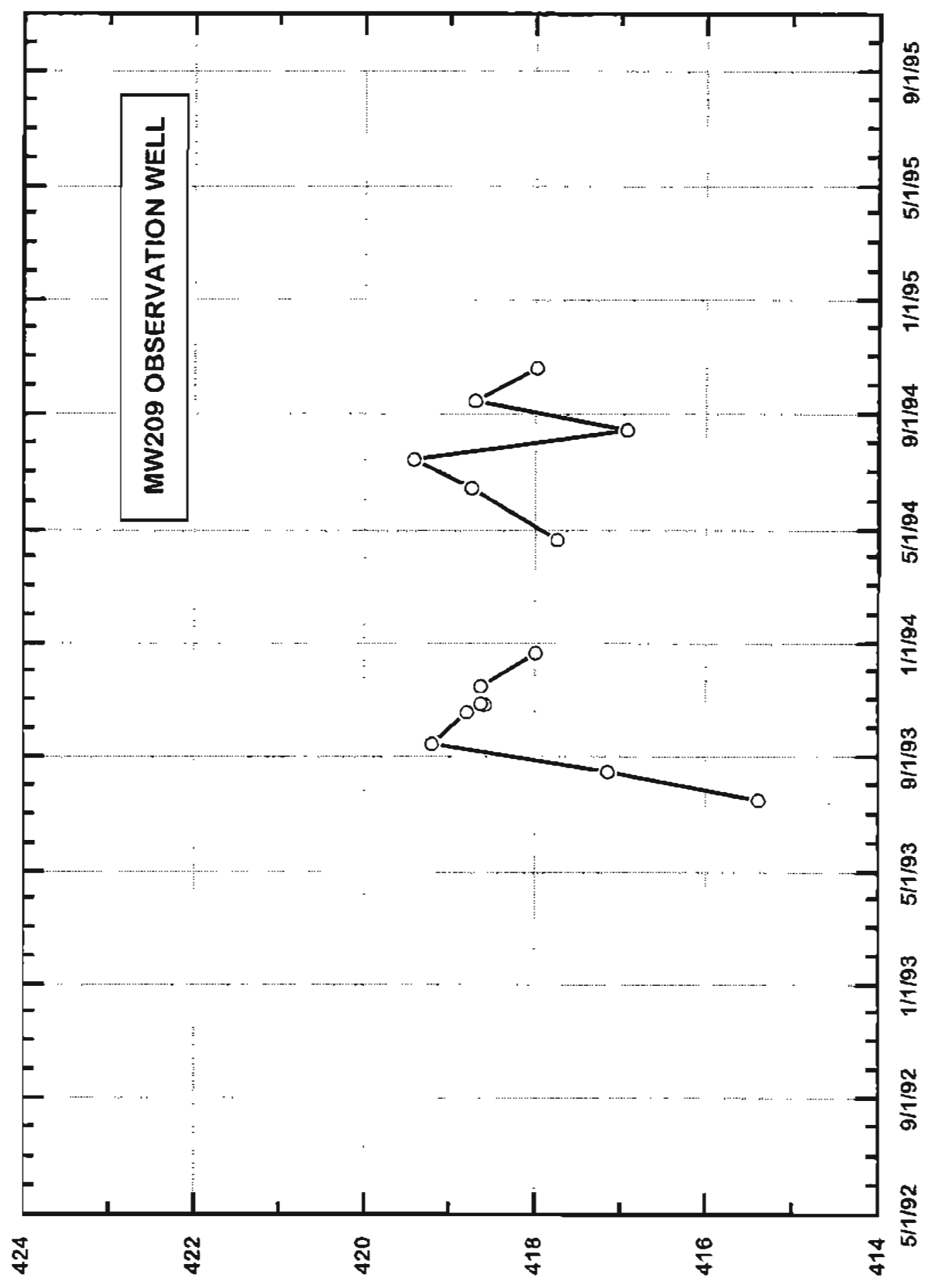

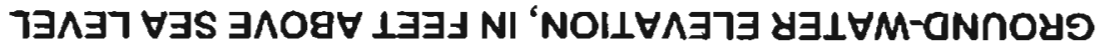



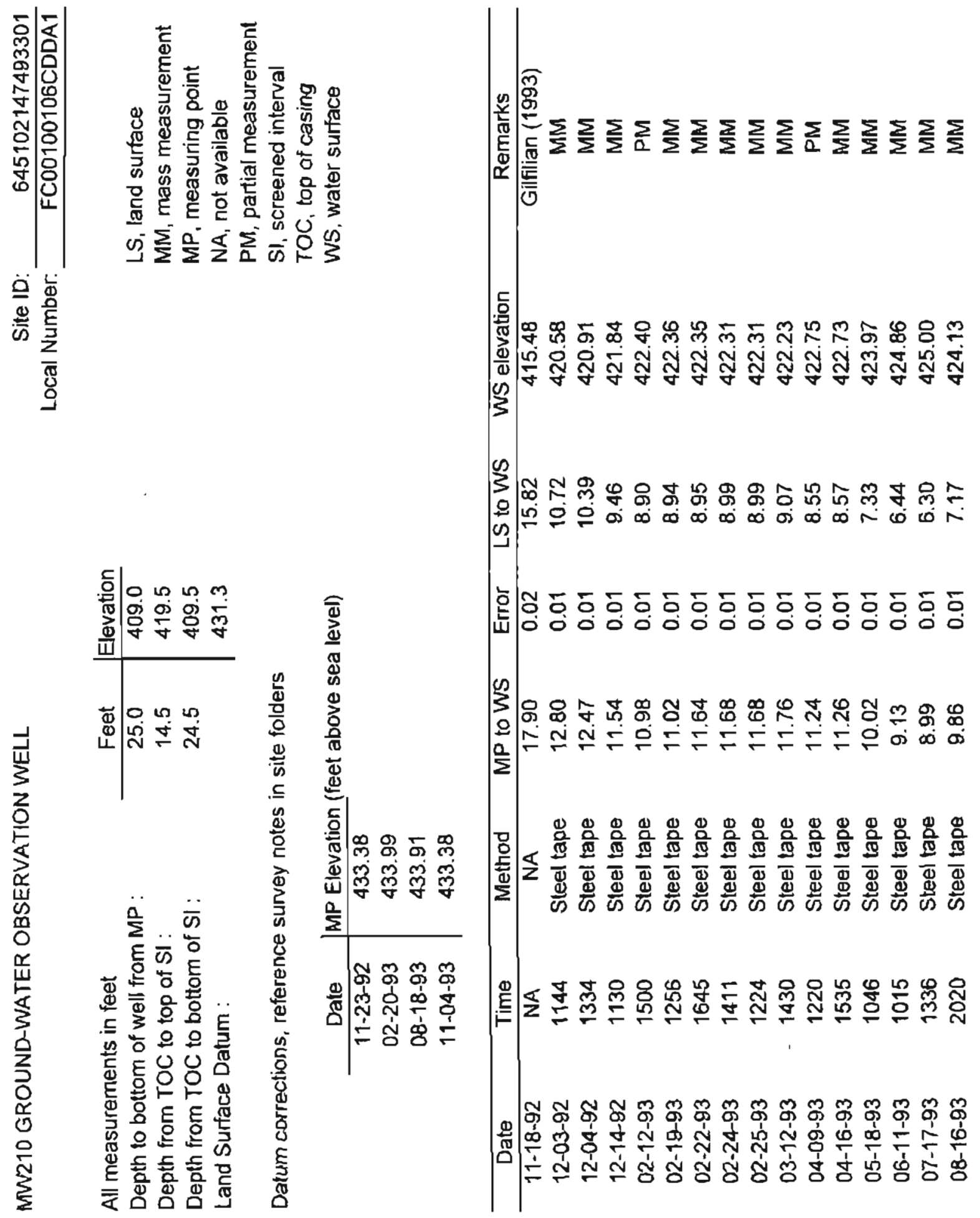


\begin{tabular}{|c|c|c|c|c|c|c|c|}
\hline \multicolumn{4}{|c|}{ MW210 GROUND-WATER OBSERVATION WELL } & \multicolumn{2}{|c|}{ Continued) } & \multirow{2}{*}{$\begin{array}{r}\text { Site ID: } \\
\text { Local Number: } \\
\text { WS elevation }\end{array}$} & \multirow{2}{*}{$\begin{array}{r}\frac{645102147493301}{\text { FC00100106CDDA1 }} \\
\text { Remarks }\end{array}$} \\
\hline Date & Time & Method & $\mathrm{MP}$ to $\mathrm{WS}$ & Error & LS to WS & & \\
\hline $09-15-93$ & 1639 & Steel tape & 10.74 & 0.01 & 8.13 & 423.47 & MM \\
\hline $10-19-93$ & 1410 & Steel tape & 10.74 & 0.01 & 8.13 & 423.17 & $M M$ \\
\hline $10-27-93$ & 1814 & E-lape & 10.91 & 0.02 & 8.30 & 423.00 & MM \\
\hline $10-28-93$ & 1144 & E-tape & 10.91 & 0.02 & 8.30 & 423.00 & MM \\
\hline $11-16-93$ & 1345 & Steel tape & 11.22 & 0.01 & 8.61 & 422.69 & MM \\
\hline $02-24-94$ & 1540 & Steel tape & 12.46 & 0.01 & 9.85 & 421.45 & MM \\
\hline $03-16-94$ & 1418 & Steel tape & 12.64 & 0.01 & 10.03 & 421.27 & MM \\
\hline $04-18-94$ & 2028 & Steel tape & 13.00 & 0.01 & 10.39 & 420.91 & $M M$ \\
\hline $04-21-94$ & 1411 & Steel tape & 12.99 & 0.01 & 10.38 & 420.92 & MM \\
\hline $06-15-94$ & 0929 & Steel tape & 13.14 & 0.01 & 10.53 & 420.77 & $M M$ \\
\hline $07-15-94$ & 1034 & Steel tape & 12.36 & 0.01 & 9.75 & 421.55 & MM \\
\hline $08-15-94$ & 1403 & Steel tape & 11.79 & 0.01 & 9.18 & 421.59 & MM \\
\hline $09-15-94$ & 1356 & Steel tape & 12.10 & 0.01 & 9.49 & 421.28 & MM \\
\hline $10-18-94$ & 1517 & Steel tape & 12.66 & 0.01 & 10.05 & 420.72 & MM \\
\hline
\end{tabular}

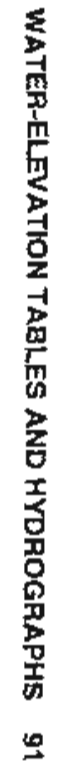




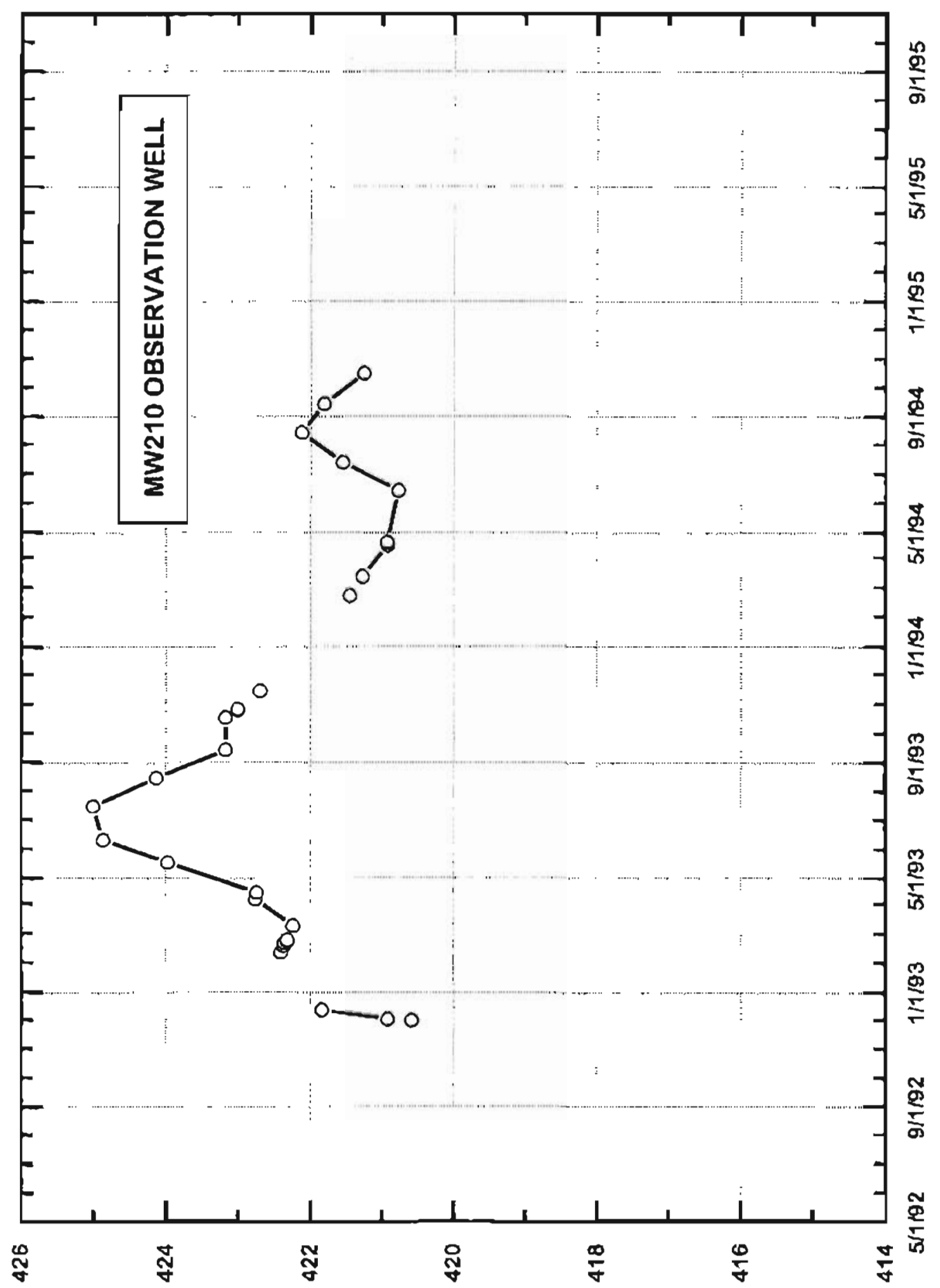

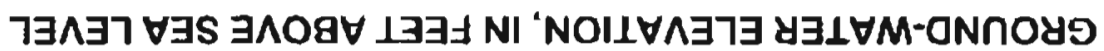


All measurements in feel

Depth to bottom of well from MP :

Depth from TOC to top of SI :

Depth from TOC to bottom of SI:

Land Surface Datum :

\begin{tabular}{c|c} 
Feet & Elevation \\
\hline 19.8 & 410.9 \\
9.1 & 421.6 \\
19.3 & 411.4 \\
& 427.79
\end{tabular}

Datum corrections, reference survey notes in site folders

\begin{tabular}{c|c} 
Date & MP Elevation (feet above sea level) \\
\hline $02-13-93$ & 430.69 \\
$02-20-93$ & 430.70 \\
$08-18.93$ & 430.48
\end{tabular}

FC00100106CDCD2

$L S_{\text {, land surface }}$

MM, mass measurement

MP, measuring point

$N A$, not avaliable

PM, partial measurement

$\mathrm{Si}$, screened interval

TOC, top of casing

WS, water surface

\begin{tabular}{|c|c|c|c|c|c|c|c|}
\hline Date & Time & Method & MP to WS & ErTor & LS to WS & WS elevation & Remaiks \\
\hline $11-18-92$ & NA & NA & 14.78 & 0.02 & $11.8 \mathrm{~B}$ & 415.91 & Giffilian (1993) \\
\hline $12-04-92$ & 1356 & Steel tape & 14.18 & 0.01 & 11.28 & 416.51 & $M M$ \\
\hline $12-14-92$ & 1645 & Steed tape & 14.46 & 0.01 & 11.56 & 416.23 & MM \\
\hline $02-10-93$ & 1415 & Steel tape & 13.78 & 0.01 & 10.88 & 416.91 & PM \\
\hline $02-13-93$ & 1420 & Steel tape & 13.89 & 0.01 & 10.99 & 416.80 & PM, instalted instr. shelter \\
\hline $02-19-93$ & 0926 & Steel lape & 13.78 & 0.07 & 10.87 & 416.92 & MM \\
\hline $02-22-93$ & 1530 & Steel tape & 13.96 & 0.01 & 11.05 & 416.74 & PM \\
\hline $02-24-93$ & 1402 & Steel tape & 13.97 & 0.01 & 11.06 & 416.73 & MM \\
\hline $02-25-93$ & 1120 & Steel tape & 13.98 & 0.01 & 11.07 & 416.72 & MM \\
\hline $03-12-93$ & 1053 & Steel tape & 14.04 & 0.01 & 11.13 & 416.66 & MM \\
\hline $04-09-93$ & 1135 & Steel tape & 14.45 & 0.01 & 11.54 & 416.25 & PM \\
\hline $04-16-93$ & 0915 & Steel tape & 13.98 & 0.01 & 11.07 & 416.72 & $M M$ \\
\hline $05-18-93$ & 1430 & Steel tape & 11.15 & 0.01 & 8.24 & 419.33 & $M M$ \\
\hline $06-11-93$ & 1030 & Steel tape & 11.72 & 0.01 & 8.81 & 418.76 & $\mathrm{MM}$ \\
\hline $07-16-93$ & 1130 & Steel tape & 12.10 & 0.01 & 9.19 & 418.38 & $\mathrm{MM}$ \\
\hline $08-16-93$ & 1045 & Steel tape & 11.60 & 0.01 & 8.69 & 418.88 & $\mathrm{MM}$ \\
\hline $09-16-93$ & 1408 & E-lape & 11.96 & 0.02 & 9.27 & 418.52 & MM \\
\hline $10-19-93$ & 1333 & Steel lape & 12.27 & 0.01 & 9.58 & 418.21 & PW \\
\hline
\end{tabular}


Site ID:

645059147494501 Local Number

FC00100106COCD2

\begin{tabular}{cccccccc}
\hline Date & Time & Method & MP to WS & Error & LS to WS & WS elevation & Remarks \\
\hline $10-28-93$ & 1158 & E-tape & 12.80 & 0.02 & 10.11 & 417.68 & PM \\
$11-16-93$ & 1659 & Steel tape & 13.14 & 0.01 & 10.45 & 417.34 & MM \\
$12-22-93$ & 1447 & E-tape & 13.17 & 0.02 & 10.48 & 417.31 & MM \\
$01-19-94$ & 1025 & E-tape & 13.78 & 0.02 & 11.09 & 416.70 & MM \\
$02-24-94$ & 1450 & Steel tape & 14.20 & 0.01 & 11.51 & 416.28 & MM \\
$03-16-94$ & 1346 & Steel tape & 15.25 & 0.01 & 12.56 & 415.23 & MM \\
$04-21-94$ & 1315 & Steel tape & 15.46 & 0.01 & 12.77 & 415.02 & MM \\
$05-16-94$ & 2145 & Steel tape & 13.23 & 0.01 & 10.54 & 417.25 & MM \\
$06-15-94$ & 0940 & Steel tape & 13.87 & 0.01 & 11.18 & 416.61 & MM \\
$07-15-94$ & 1005 & Steel tape & 11.57 & 0.01 & 8.88 & 418.91 & MM \\
$08-15-94$ & 1014 & Steel tape & 11.18 & 0.01 & 8.49 & 419.30 & MM \\
$09-15-94$ & 1337 & Steel tape & 12.39 & 0.01 & 9.70 & 418.09 & MM \\
$10-18-94$ & 1622 & Steel tape & 13.94 & 0.01 & 11.25 & 416.54 & MM \\
\hline
\end{tabular}




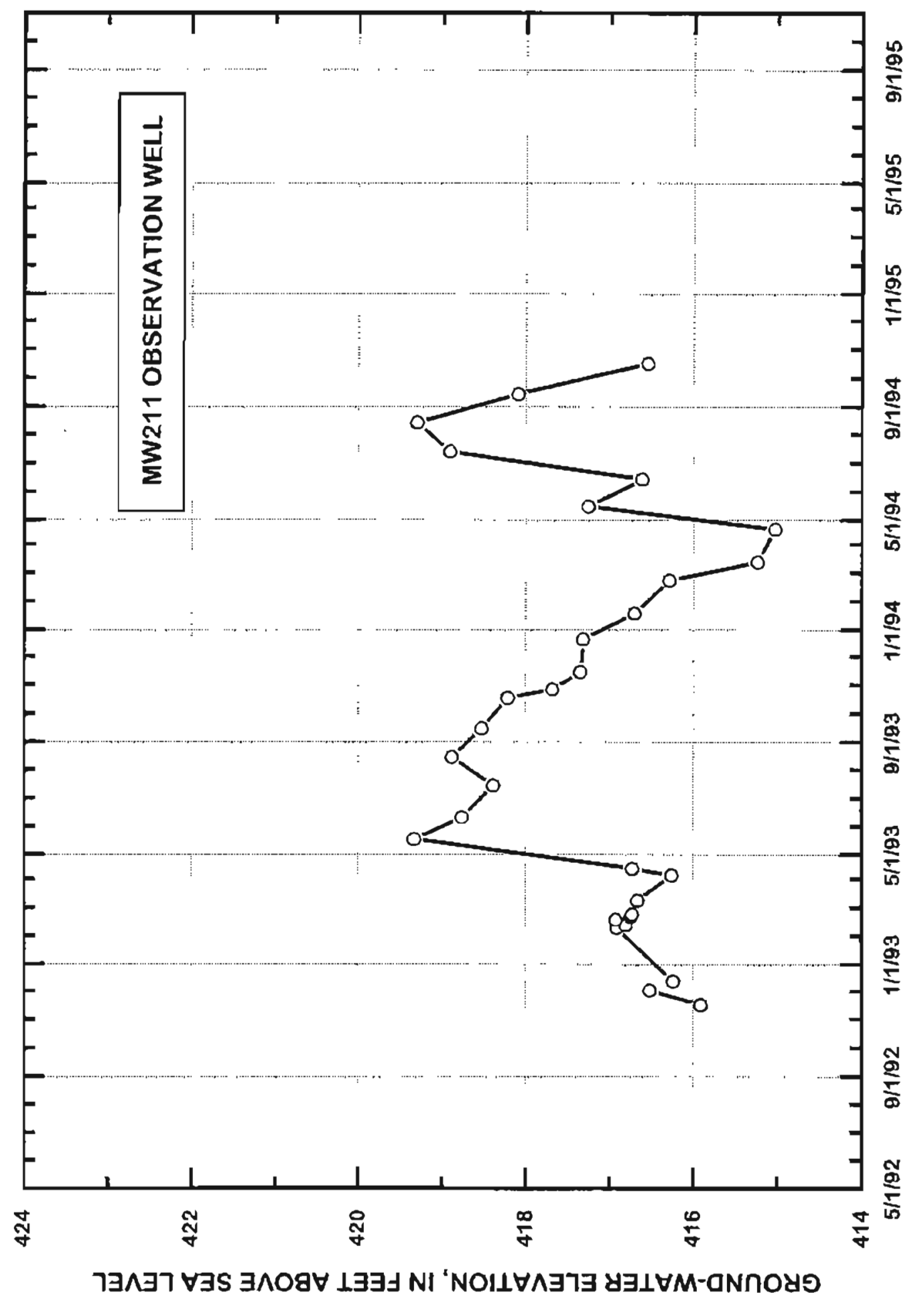


Site ID: Local Number

\begin{tabular}{c|c} 
Feet & Elevation \\
\hline 23.9 & 411.0 \\
21.2 & 413.7 \\
23.4 & 411.5 \\
& 432.75
\end{tabular}

All measurements in feet

Depth to bottom of well from MP:

Depth from TOC to top of $\$ I$ :

Depth from TOC to bottom of SI :

Land Surface Datum :

Datum corrections, reference survey notes in site folders

\begin{tabular}{c|c} 
Date & MP Elevation (feet above sea level) \\
\hline $02-13-93$ & 434.91 \\
$02-13-93$ & 434.88 \\
$08-18-93$ & 434.77
\end{tabular}

645103147494001

FC00100106CDDB4

LS, land surface

$M M$, mass rneasurement

$M P$, measuring point

NA, not available

PM, partial measurement

PT, pump test

SI, screened interval

TOC, top of casing

WS, water surface

\begin{tabular}{|c|c|c|c|c|c|c|c|}
\hline Date & Time & Method & MP to WS & Error & LS to WS & WS elevation & Remarks \\
\hline $11-23-92$ & 1416 & Steel tape & 19.98 & 0.01 & 17.82 & 414.93 & $\mathrm{PM}$ \\
\hline $12-04-92$ & 1410 & Steel tape & 19.61 & 0.01 & 17.45 & 415.30 & MM \\
\hline $12-14-92$ & 1440 & Steel tape & 19.48 & 0.01 & 17.32 & 415.43 & $\mathrm{MM}$ \\
\hline $01-15-93$ & 1605 & Steel tape & 18.43 & 0.01 & 16.27 & 416.48 & PM \\
\hline $02-10-93$ & 1448 & Stéel tape & 18.64 & 0.01 & 16.48 & 416.27 & PM \\
\hline $02-13-93$ & 1540 & Steel tape & 18.73 & 0.01 & 16.60 & 416.15 & PM, installed instr, shelter \\
\hline $02-19-93$ & 1000 & Steel tape & 18.60 & 0.01 & 16.47 & 416.28 & MM \\
\hline $02-20-93$ & 1000 & Steel tape & 18.63 & 0.01 & 16.50 & 416.25 & MM, installed CR10 \\
\hline $02-22-93$ & 1557 & Steel tape & 18.47 & 0.01 & 16.34 & 416.41 & MM \\
\hline $02-24-93$ & 1019 & Steel tape & 18.57 & 0.01 & 16.44 & 416.31 & PM \\
\hline $02-25-93$ & 1139 & Steel tape & 18.31 & 0.01 & 16.18 & 416.57 & MM \\
\hline $03-12-93$ & 1205 & Steel tape & 18.57 & 0.01 & 16.44 & 416.31 & MM \\
\hline $04-16-93$ & 0902 & Steel tape & 18.37 & 0.01 & 16.24 & 416.51 & MM \\
\hline $04-30-93$ & 1650 & Steel tape & 16.87 & 0.01 & 14.74 & 418.01 & PM \\
\hline $05-12-93$ & 1059 & E-tape & 15.97 & 0.02 & 13.84 & 418.91 & $P M$ \\
\hline $05-18-93$ & 1500 & Steel tape & 15.85 & 0.01 & 13.72 & 418.92 & MM \\
\hline $06-11-93$ & 1021 & Steel tape & 16.35 & 0.01 & 14.22 & 418.42 & MM \\
\hline $07-16-93$ & 1102 & Steel tape & 16.58 & 0.01 & 14.45 & 418.19 & MM \\
\hline
\end{tabular}


Site ID:

645103147494001

Local Number:

FC00100106CDDB4

\begin{tabular}{|c|c|c|c|c|c|c|c|}
\hline Date & Time & Method & MP to WS & Error & LS to WS & WS elevation & Remarks \\
\hline $08-16-93$ & 1020 & Steel tape & 16.34 & 0.01 & 14.21 & 418.43 & MM \\
\hline $09-16-93$ & 1413 & E-tape & 16.83 & 0.02 & 14.81 & 417.94 & MM \\
\hline $10-19-93$ & 1321 & Steel tape & 17.09 & 0.01 & 15.07 & 417.68 & PM \\
\hline $10-27-93$ & 1650 & E-tape & 16.39 & 0.02 & 14.37 & 418.38 & PM \\
\hline $10-28-93$ & 1335 & E-tape & 17.23 & 0.02 & 15.21 & 417.54 & PM \\
\hline $11-16-93$ & 1604 & Steel tape & 17.95 & 0.01 & 15.93 & 416.82 & MM \\
\hline $12-22-93$ & 1541 & E-tape & 17.68 & 0.02 & 15.66 & 417.09 & MM \\
\hline $01-05-94$ & 1031 & Eulape & 17.69 & 0.02 & 15.67 & 417.08 & $P M$ \\
\hline $01-19-94$ & 1041 & Sleel tape & 18.39 & 0.01 & 16.37 & 416.38 & MM \\
\hline $02-22-94$ & 0903 & Steel tape & 18.73 & 0.01 & 16.71 & 416.04 & MM \\
\hline $03-16-94$ & 1349 & Steel tape & 18.78 & 0.01 & 16.76 & 415.99 & MM \\
\hline $04-14-94$ & 1349 & Steel tape & 18.78 & 0.01 & 16.76 & 415.99 & PM \\
\hline $04-21.94$ & 1338 & Steel tape & 19.91 & 0.01 & 17.89 & 414.86 & MM \\
\hline $05-16-94$ & 2120 & Steel tape & 17.59 & 0.01 & 15.57 & 417.18 & MM \\
\hline $06-15-94$ & 0930 & Steel tape & 18.58 & 0.01 & 16.56 & 416.19 & MM \\
\hline $07-15-94$ & 0935 & Steel tape & 18.20 & 0.01 & 16.18 & 416.57 & MM \\
\hline $07-21-94$ & 1036 & Steel tape & 16.28 & 0.01 & 14.26 & 418.49 & PM \\
\hline $07-22-94$ & 1352 & Sleel tape & $\{6.50$ & 0.01 & 14.48 & 418.27 & $P M$ \\
\hline $08-15-94$ & 0950 & Steel tape & 15.93 & 0.01 & 13.91 & 418.84 & $M M$ \\
\hline $09-15-94$ & 1426 & Steel tape & $\$ 7.10$ & 0.01 & 15.08 & 417.67 & MM \\
\hline $09-29-94$ & 1310 & Steel lape & 17.95 & 0.01 & 15.93 & 416.82 & PM \\
\hline $10-18-94$ & 1549 & Steel tape & 18.54 & 0.01 & 16.52 & 416.23 & MM \\
\hline $10-25-84$ & 1320 & Steel tape & 18.82 & 0.01 & 16.80 & 415.95 & PM \\
\hline $02-17-95$ & 1332 & Steel tape & 19.28 & 0.01 & 17.26 & 415.49 & PM \\
\hline $02-27-95$ & 0910 & Steel tape & 18.51 & 0.01 & 16.35 & 416.40 & PT \\
\hline $03-14-95$ & 1503 & Steel tape & 19.13 & 0.01 & 16.97 & 415.78 & PT \\
\hline $04-18-95$ & 1144 & Steel tape & 19.08 & 0.01 & 16.92 & 415.83 & $\mathrm{PM}$ \\
\hline $05-26-95$ & 1035 & Steel tape & 17.25 & 0.01 & 15.09 & 417.66 & PM \\
\hline $06-05-95$ & 1614 & Steel tape & 17.46 & 0.01 & 15.30 & 417,45 & PM \\
\hline $07-17-95$ & 1311 & Steel tape & 16.12 & 0.01 & 13.96 & 418.79 & MM \\
\hline $08-16-95$ & 1538 & Steet tape & 16.43 & 0.01 & 14.27 & 418.48 & MKA \\
\hline
\end{tabular}




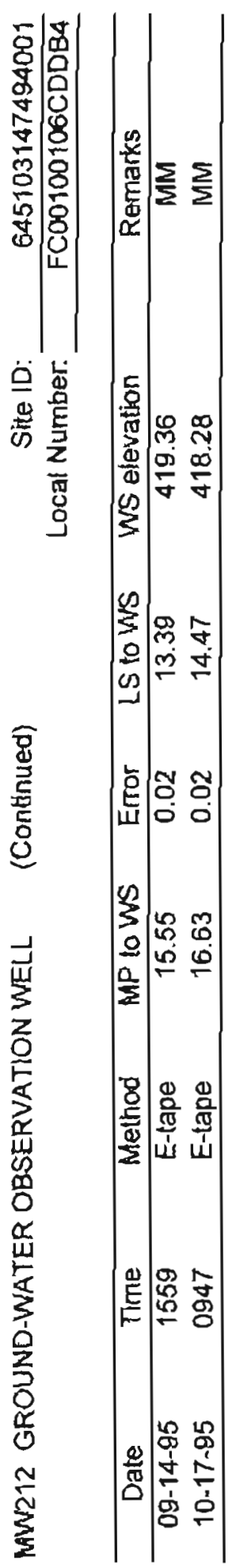




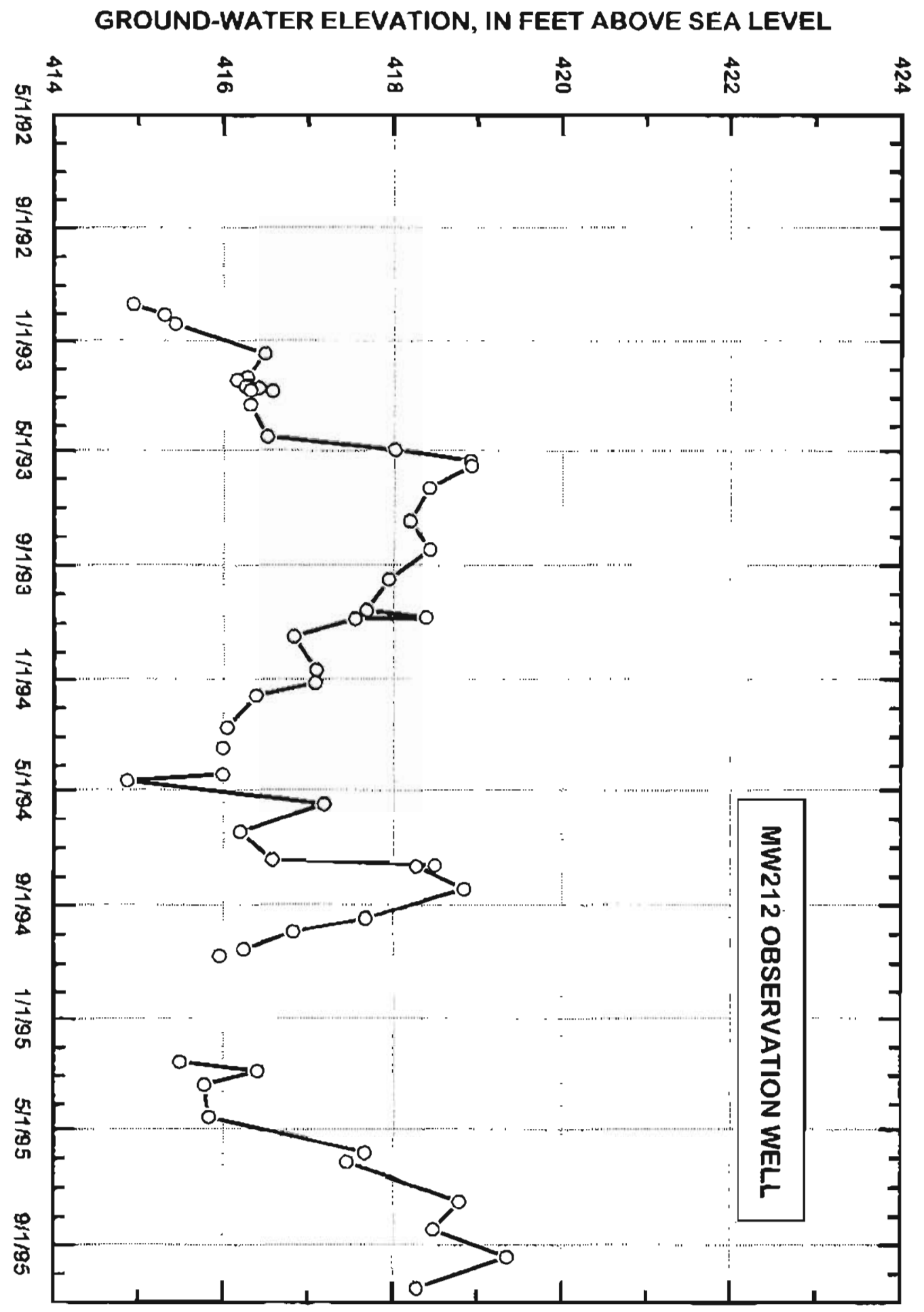


Site ID: Local Number:

\begin{tabular}{c|c} 
Feet & Elevalion \\
\hline 23.9 & 409.8 \\
21.2 & 412.5 \\
23.5 & 410.1 \\
& 431.54
\end{tabular}

Datum corrections, reference survey notes in site folders

\begin{tabular}{c|c} 
Date & MP Elevation (feet above sea level) \\
\hline $02-13-93$ & 433.74 \\
$02-13-93$ & 433.71 \\
$08-18-93$ & 433.61
\end{tabular}

All measurements in feet

Depth to bottom of well from MP :

Depth from TOC to top of $\mathrm{SI}$ :

Depth from $7 \mathrm{OC}$ to bottom of SI :

Land Surface Datum :

(2) -433.93
r:

$\frac{645104147494501}{\text { FC00100106CDCA1 }}$

\section{LS, land surface} MM, mass measurement MP, measuring point NA, nol available PM, partial measurement S!, screened interval TOC, top of casing WS, water surface

\begin{tabular}{|c|c|c|c|c|c|c|c|}
\hline Date & Time & Hethod & MP to MS & Error & LS to WS & WS etevation & Remarks \\
\hline $11-23-92$ & 1432 & Steel tape & 18.79 & 0.01 & 16.59 & $4 \overline{14.95}$ & PMA \\
\hline $12-04-92$ & 1415 & Steel tape & 18.52 & 0.01 & 16.32 & 415.22 & MM \\
\hline $12-14-92$ & 1425 & Steel tape & 18.40 & 0.01 & 16.20 & 415.34 & MM \\
\hline $01-15-93$ & 1625 & Steel tape & 17.31 & 0.01 & 15.11 & 416.43 & $P M$ \\
\hline $02-13-93$ & 1545 & Steel tape & 17.59 & 0.01 & 15.42 & 416.12 & PM, installed instr. shelter \\
\hline $02-19-93$ & 0958 & Steel tape & 17.50 & 0.01 & 15.33 & 416.21 & $\mathrm{MM}$ \\
\hline $02-20-93$ & 1034 & Steel tape & 17.52 & 0.01 & 15.35 & 416.19 & MM, installed CR10 \\
\hline $02-22-93$ & 1559 & Steel tape & 17.29 & 0.01 & 15.12 & 416.42 & MM \\
\hline $02-24-93$ & 1008 & Steel tape & 17.41 & 0.01 & 15.24 & 416.30 & PM \\
\hline $02-25-93$ & 1159 & Steel tape & 17.12 & 0.01 & 14.95 & 416.59 & MA \\
\hline $03-12-93$ & 1221 & Steel tape & 17.44 & $0: 01$ & 15.27 & 416.27 & $M M$ \\
\hline $04-16-93$ & 0912 & Steel tape & 17.23 & 0.01 & 15.06 & 416.48 & $M M$ \\
\hline $04-30-93$ & 1700 & Steel tape & 15.78 & 0.01 & 13.61 & 417.93 & PAM \\
\hline $05-12-93$ & 1055 & E-tape & 14.86 & 0.02 & 12.69 & 418.85 & PM \\
\hline $05-18-93$ & 1445 & Steel tape & 14.64 & 0.01 & 12.47 & 418.97 & $M M$ \\
\hline $06-11-93$ & 1016 & Steel tape & 15.19 & 0.01 & 13.02 & 418.42 & MM \\
\hline $07-16-93$ & 1057 & Steel tape & 16.05 & 0.01 & 13.88 & 417.56 & MM \\
\hline $08-16-93$ & 1016 & Steel tape & 15.23 & 0.01 & 13.06 & 418.38 & MM \\
\hline
\end{tabular}


MW213 GROUND-WATER OBSERVATION WELL (Continued)

Site ID:

645104147494501

Local Number:

FC00100106CDCA1

\begin{tabular}{cccccccc}
\hline Date & Time & Method & MP to WS & Error & LS to WS & WS elevation & Remarks \\
\hline $10-19-93$ & 1318 & Steel tape & 15.92 & 0.01 & 13.85 & 417.69 & MM \\
$10-27-93$ & 1655 & E-tape & 16.21 & 0.02 & 14.14 & 417.40 & PM \\
$10-28-93$ & 1322 & E-tape & 16.04 & 0.02 & 13.97 & 417.57 & PM \\
$11-16-93$ & 1606 & Steel tape & 16.81 & 0.01 & 14.74 & 416.80 & MM \\
$12-22-93$ & 1552 & E-tape & 16.54 & 0.02 & 14.47 & 417.07 & PM \\
$01-04-94$ & 1658 & E-tape & 16.54 & 0.02 & 14.47 & 417.07 & PM \\
$01-19-94$ & 1104 & E-tape & 17.25 & 0.02 & 15.18 & 416.36 & MM \\
$02-22-94$ & 0920 & Steel tape & 17.63 & 0.01 & 15.56 & 415.98 & MM, removed CR10 \\
$03-16-94$ & 1354 & Steel tape & 17.67 & 0.01 & 15.60 & 415.94 & MM \\
$04-21-94$ & 1347 & Steel tape & 17.77 & 0.01 & 15.70 & 415.84 & MM \\
$05-16-94$ & 2113 & Steel tape & 16.44 & 0.01 & 14.37 & 417.17 & MM \\
$06-15-94$ & 0921 & Steel tape & 17.44 & 0.01 & 15.37 & 416.17 & MM \\
$07-15-94$ & 0940 & Steel tape & 15.12 & 0.01 & 13.05 & 418.49 & MM \\
$08-15-94$ & 0955 & Steel tape & 14.84 & 0.01 & 12.77 & 418.77 & MM \\
$09-15-94$ & 1330 & Steel lape & 15.92 & 0.01 & 13.85 & 417.69 & MM \\
$10-18-94$ & 1545 & Steel tape & 17.38 & 0.01 & 15.31 & 416.23 & MM \\
$10-20-94$ & 1327 & Steel tape & 17.60 & 0.01 & 15.40 & 416.14 & MM \\
\hline
\end{tabular}




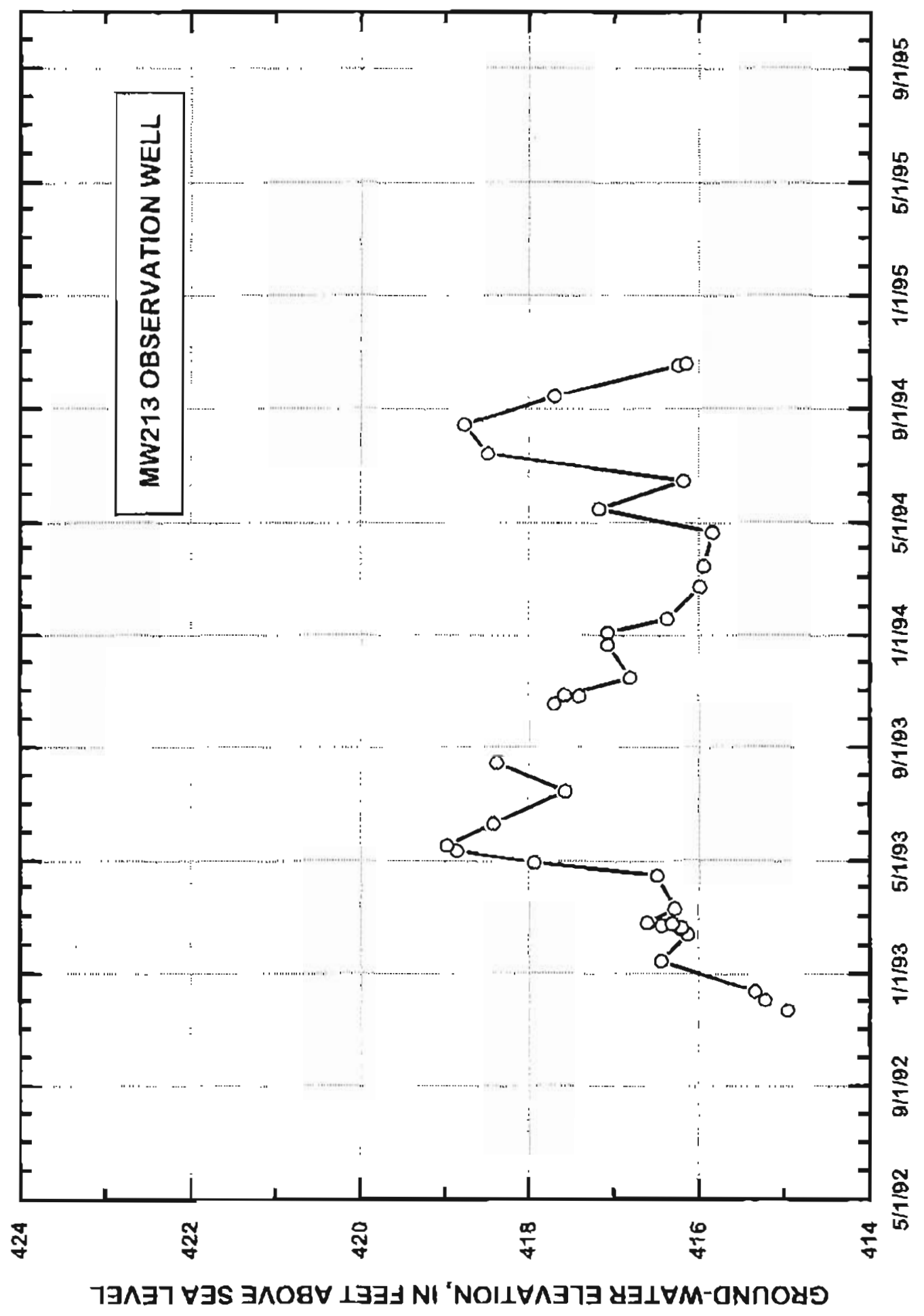




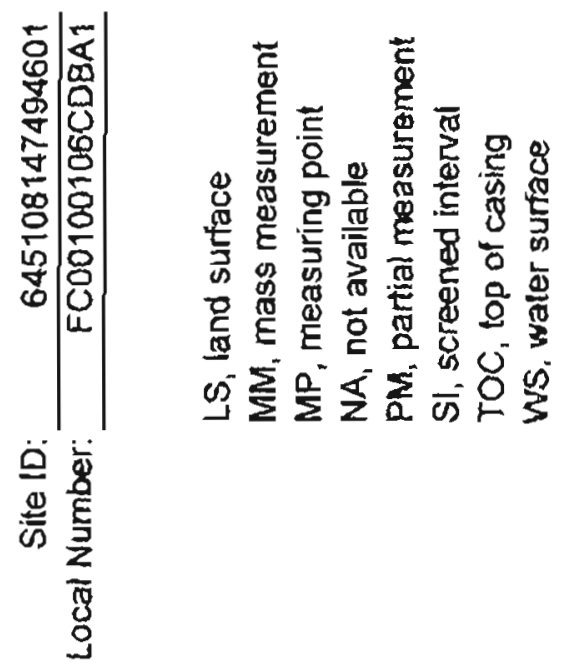

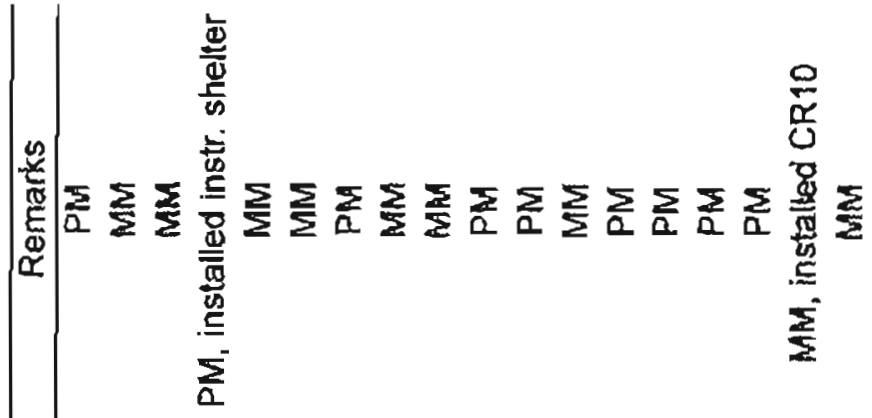

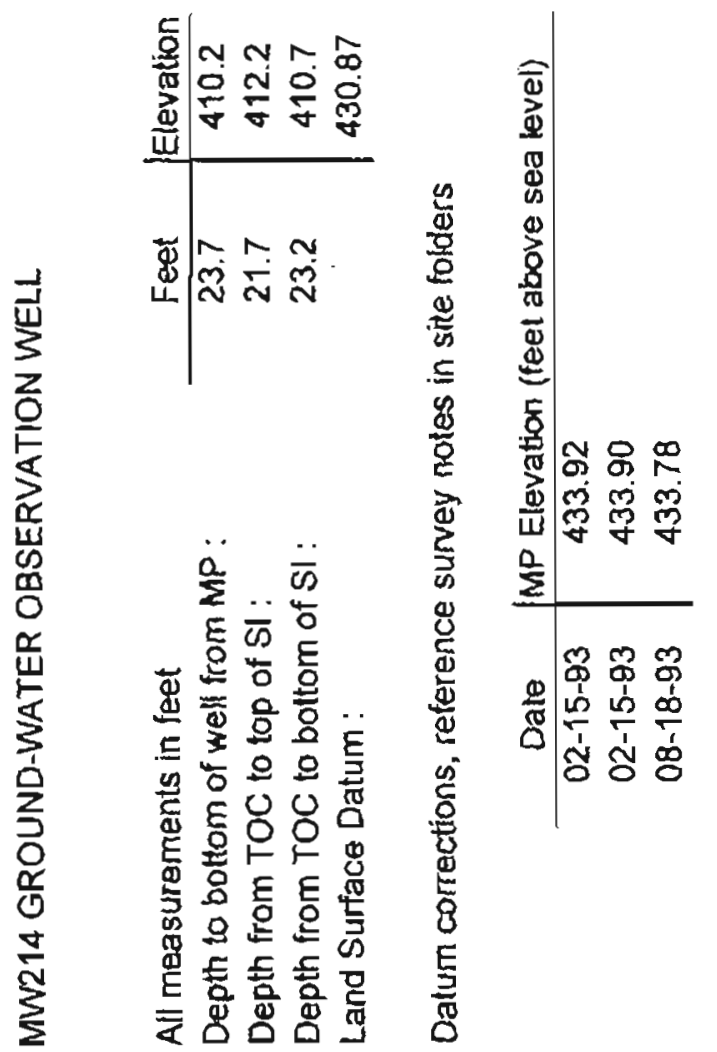

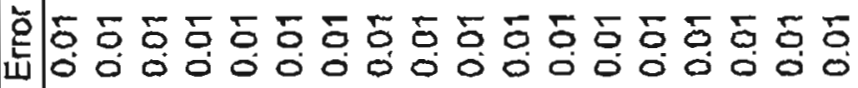

क)

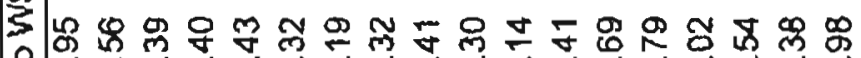

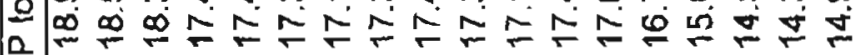
$\frac{2}{2}$

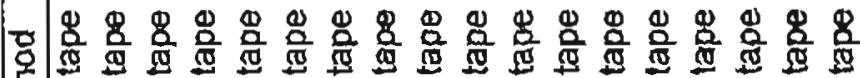

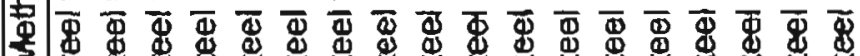

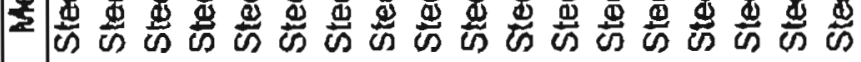

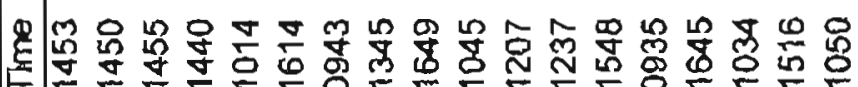

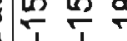

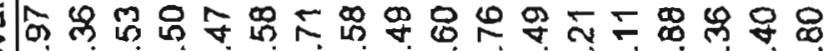

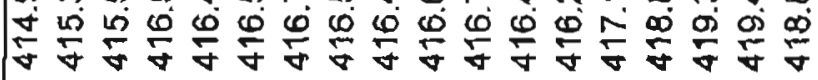

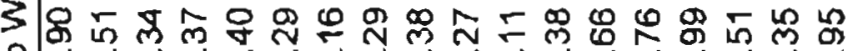

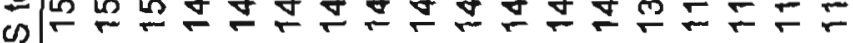




\begin{tabular}{|c|c|c|c|c|c|c|c|c|}
\hline & MW214 GRC & WAT & BSERVATKO & WELL & ontinue & & Sile lo: & 645108147494601 \\
\hline & Date & THine & Method & MP to WS & Efror & LS to WS & WS elevation & Remarks \\
\hline & $07-01-93$ & 1250 & E-tape & 15.40 & 0.02 & 12.37 & 418.38 & $\mathrm{PM}$ \\
\hline & $07-16-93$ & 1044 & Steel tape & 15.70 & 0.02 & 12.67 & 418.08 & MM \\
\hline & $08-16-93$ & 1005 & Steel tape & 15.18 & 0.01 & 12.15 & 418.60 & MM \\
\hline & $09-16-93$ & 1422 & E-tape & 15.46 & 0.02 & 12.55 & 418.32 & MM \\
\hline & $10-19-93$ & 1300 & Steel tape & 15.68 & 0.01 & 12.67 & 418.20 & MM \\
\hline & $10-27-93$ & 1752 & E-tape & 15.93 & 0.02 & 13.02 & 417.85 & PM \\
\hline & $10-28-93$ & 1314 & E-tape & 15.83 & 0.02 & 12.92 & $4 \uparrow 7.95$ & $P M$ \\
\hline & $11-16-93$ & 1545 & Steel tape & 16.54 & 0.01 & 13.63 & 417.24 & MM \\
\hline & $12-07-93$ & 1649 & Steel tape & 16.41 & 0.01 & $\uparrow 3.50$ & 417.37 & PM \\
\hline & $12-08-93$ & 1537 & E-tape & 16.63 & 0.02 & 13.72 & 417.15 & PM \\
\hline & $12-09-93$ & 0925 & Steel tape & 16.63 & 0.01 & 13.72 & 417.15 & PM \\
\hline & $12-22-93$ & 1258 & E-tape & 16.50 & 0.02 & 13.59 & 417.28 & $M M$ \\
\hline & $01-19-94$ & 1117 & Etape & 17.00 & 0.02 & 14.09 & 416.78 & MM \\
\hline & $02-22-94$ & 1019 & Steel tape & 17.49 & 0.01 & 14.58 & 416.29 & MM, pulled CR10 \\
\hline & $03-16-94$ & 1401 & Steel tape & 17.51 & 0.01 & 14.60 & 416.27 & MM \\
\hline & $04-21-94$ & 1431 & Steel tape & 17.60 & 0.01 & 14.69 & 416.18 & MA \\
\hline & $05-16-94$ & 2226 & Steel tape & 16.26 & 0.01 & 13.35 & 417.52 & MM \\
\hline & $06-15-94$ & 1023 & Steel tape & 17.18 & 0.01 & 14.27 & 416.60 & MM \\
\hline & $07-15-94$ & 0850 & Steel tape & 15.22 & 0.01 & 12.31 & 418.56 & $M M$ \\
\hline & $08-15-94$ & 0925 & Steel tape & 14.92 & 0.01 & 12.01 & 418.86 & MM \\
\hline & $09-15-94$ & 1414 & Steel tape & 15.64 & 0.01 & 12.73 & 418.14 & MM \\
\hline
\end{tabular}




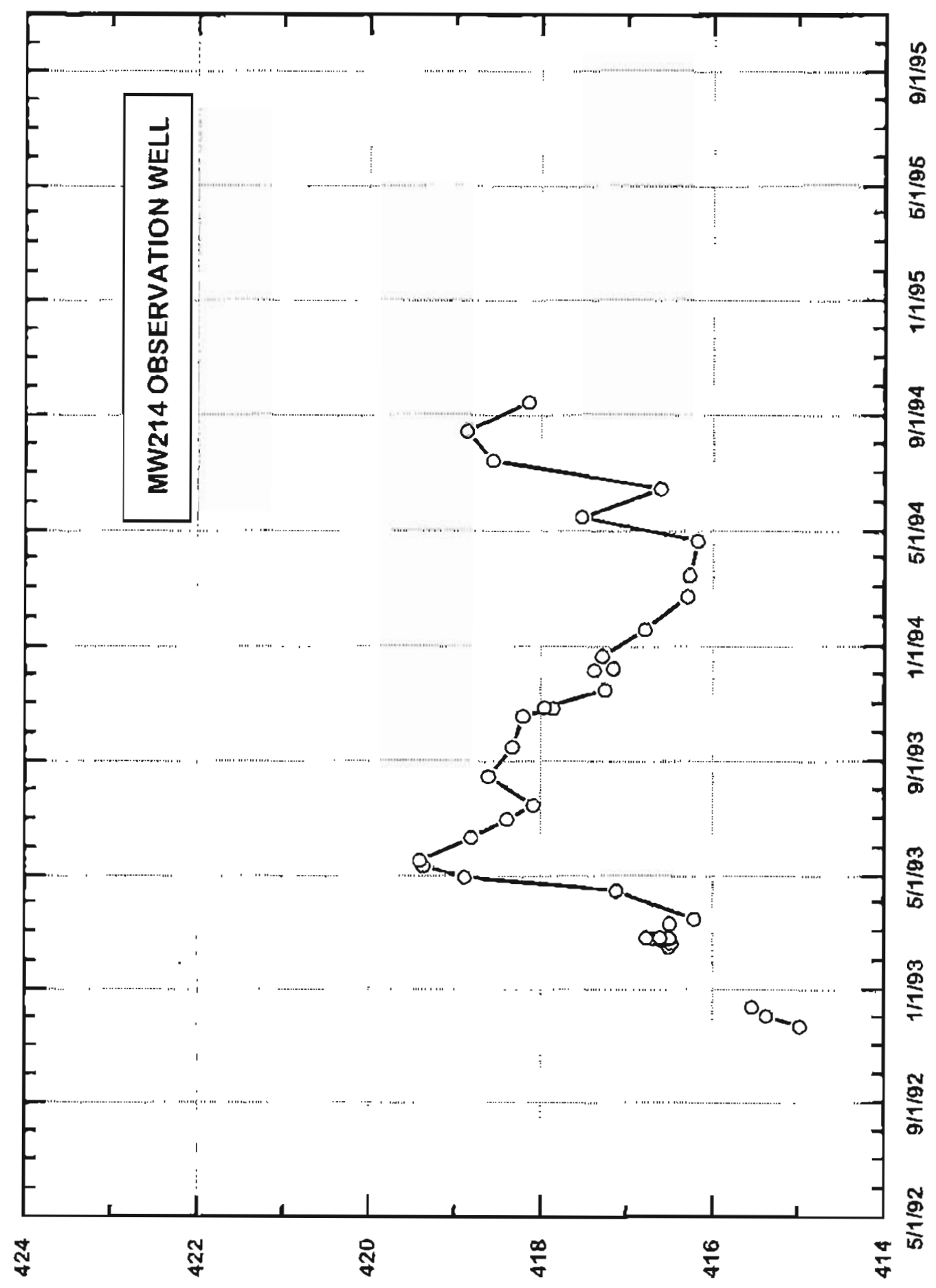

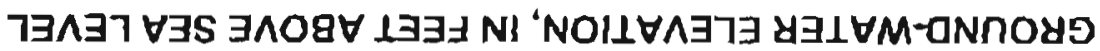


Site ID: Local Number. 645111147483601 C00100105CBCC1

All measurements in feet

Depth to botton of well from MP :

Depth from TOC to top of SI:

Depth from TOC to bottom of SI:

Land Surface Datum :

\begin{tabular}{c|c} 
Feet & Elevation \\
\hline NA & NA \\
NA & NA \\
NA & NA \\
& 433.5
\end{tabular}

Datum corrections, reference survey notes in site loiders

$$
\begin{array}{c|c}
\text { Date } & \text { MP elevation (feet above sea level) } \\
\hline 11-02-93 & 435.7
\end{array}
$$

\section{LS, Jand surface} $M M$, mass measuremen! $M P$, measuring point $N A$, not available $P M$, partial measurement SI, screened interval TOC, top of casing WS, water surface

\begin{tabular}{cccccccc}
\hline Date & Time & Method & MP to WS & Error & LS to WS & WS elevation & Remarks \\
\hline $01-20-94$ & 1410 & E-tape & 16.92 & 0.02 & 14.75 & 418.78 & MM \\
$03-16-94$ & 1459 & Steel tape & 17.62 & 0.01 & 15.45 & 418.08 & MM \\
$04-21-94$ & 1328 & Steel tape & 17.78 & 0.01 & 15.61 & 417.92 & MM \\
$06-15-94$ & 0959 & Steel tape & 17.24 & 0.01 & 15.07 & 418.46 & MM \\
$07-15-94$ & 0833 & Steel tape & 15.22 & 0.01 & 13.05 & 420.48 & MM \\
$08-15-94$ & 1110 & Steel tape & 15.31 & 0.01 & 13.14 & 420.39 & MM \\
$09-15-94$ & 1218 & Steel tape & 15.94 & 0.01 & 13.77 & 419.76 & MM \\
$10-18-94$ & 1434 & Steel tape & 17.01 & 0.01 & 14.84 & 418.69 & MM \\
$02-16-95$ & 1505 & Steel tape & 18.03 & 0.01 & 15.86 & 417.67 & MM \\
$07-18-95$ & 1126 & Steel tape & 14.43 & 0.01 & 12.26 & 421.27 & MM \\
$08-15-95$ & 1403 & E-tape & 15.75 & 0.02 & 13.58 & 419.95 & MM \\
$09-14-95$ & 1143 & E-tape & 14.57 & 0.02 & 12.40 & 421.13 & MM \\
$10-17-95$ & 1057 & E-tape & 15.42 & 0.02 & 13.25 & 420.28 & MM
\end{tabular}




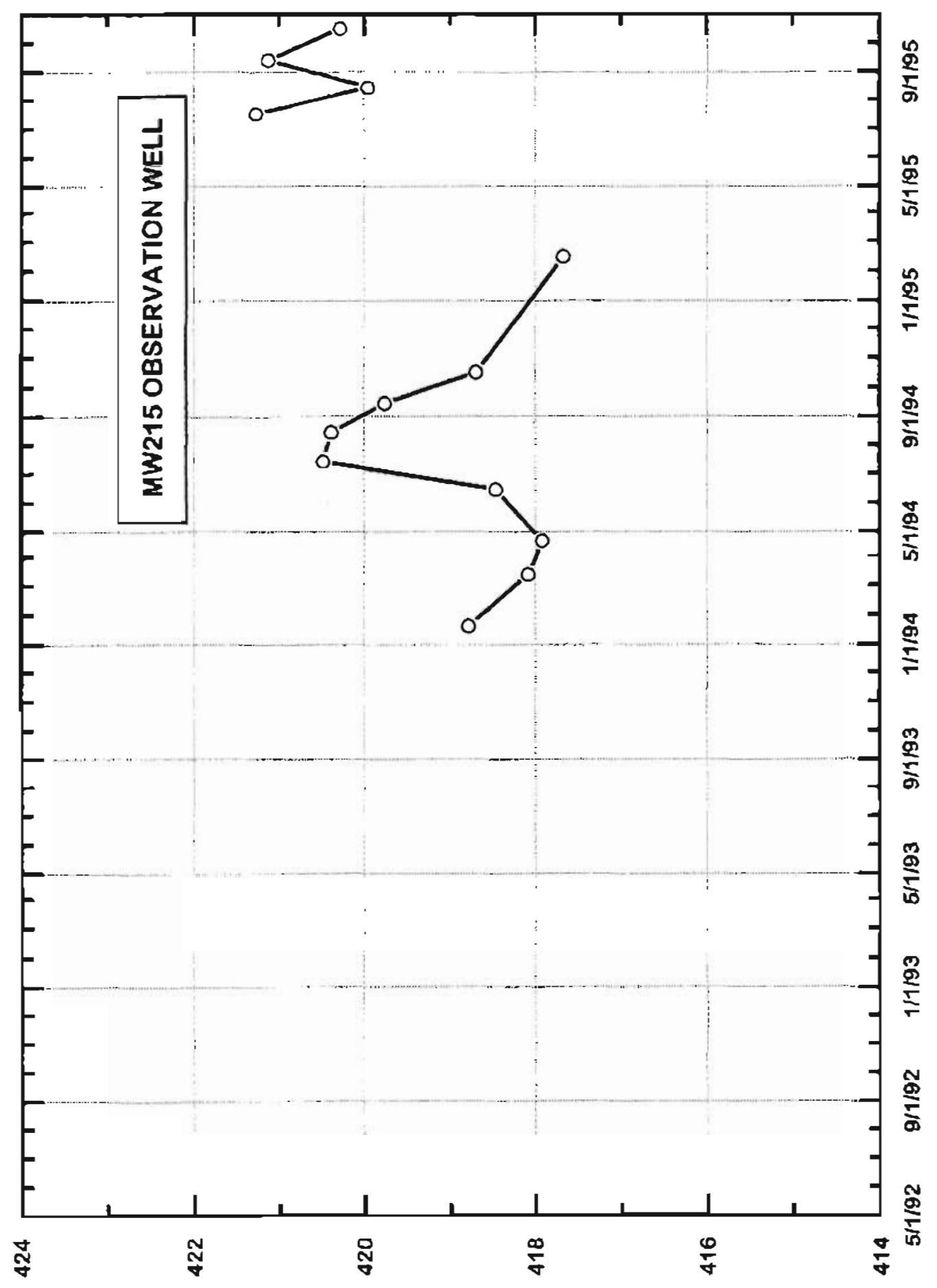

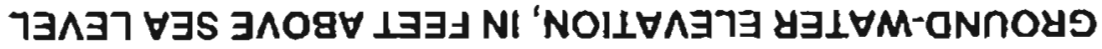


All measurements in feet

Depth to bottom of well from MP

Depth from TOC to top of SI:

Depth from TOC to bottom of $\mathrm{SI}$ :

\begin{tabular}{c|c} 
Feet & Elevation \\
\hline 60.6 & 372.2 \\
50.6 & 382.2 \\
60.6 & 372.2 \\
& 429.5
\end{tabular}

Land Surface Datum :

Datum corrections, reference survey notes in sile folders

$$
\begin{array}{c|c}
\text { Date } & \text { MP elevation (feet above sea level) } \\
\hline 01-23-95 & 432.81
\end{array}
$$

FC00100106CDDC2

$L S$, land surface

MM, mass measurement

$M P$, measuring point

NA, not available

PM, partial measurement

$\mathrm{SI}$, screened interval

TOC, top of casing

WS, water surface

\begin{tabular}{cccccccc}
\hline Date & Time & Method & MP to WS & Error & LS to WS & WS elevation \\
\hline $08-15-94$ & 1959 & Steel tape & 13.40 & 0.01 & 10.09 & 419.41 & Remarks \\
$09-15-94$ & 1133 & Steel tape & 14.76 & 0.01 & 11.45 & 418.05 & MM \\
$09-29-94$ & 1325 & Steel tape & 15.60 & 0.01 & 12.29 & 417.21 \\
$10-18-94$ & 1617 & Steel tape & 16.22 & 0.01 & 12.91 & 416.59 & PM \\
$07-17-95$ & 1408 & Steel tape & 13.50 & 0.01 & 10.19 & 419.31 & MM \\
\hline
\end{tabular}




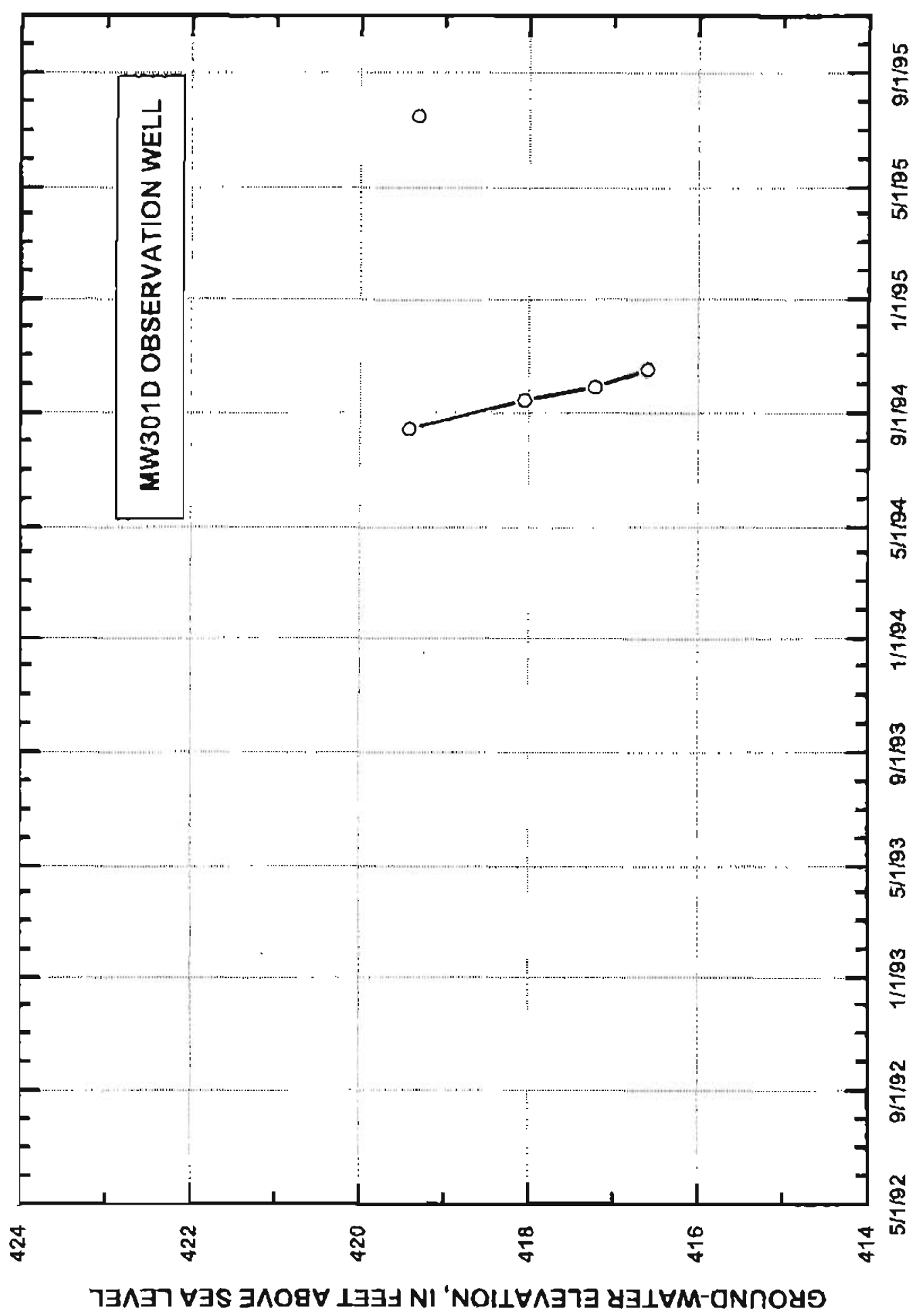


Site 10: Local Number: 645059147494002 FC00100106CDDC3

All measurements in feet Depth to bottom of well from MP : Depth from TOC to top of $\mathrm{S1}$ : Depth from TOC to bottom of $S 1$ : Land Surface Datum:

\begin{tabular}{c|c} 
Feet & Elevation \\
\hline 17.1 & 415.3 \\
7.1 & 425.3 \\
17.1 & 415.3 \\
& 429.5
\end{tabular}

Dahum corrections, reference survey notes in site folders

$$
\begin{array}{c|c}
\text { Date } & \text { MP elevation (feet above sea level) } \\
\hline 01-23-95 & 432.44
\end{array}
$$

LS, land surface

MM. mass measurement

MP, measuring point

NA, not available

PM, partial measurement

SI, screened interval

TOC, top of casing

WS, water surface

\begin{tabular}{cccccccc}
\hline Date & Time & Method & MP to WS & Error & LS to WS & WS elevation & Remarks \\
\hline $08-15-94$ & 2009 & Steel tape & 13.02 & 0.01 & 10.08 & 419.42 & MM \\
$09-15-94$ & 1438 & Steel tape & 14.35 & 0.01 & 11.41 & 418.09 & MM \\
$09-29-94$ & 1329 & Steel tape & 15.20 & 0.01 & 12.26 & 417.24 & PM \\
$10-20-94$ & 1236 & Steel tape & 15.93 & 0.01 & 12.99 & 416.51 & MM \\
$07-17-95$ & 1405 & Steel tape & 13.15 & 0.01 & 10.21 & 419.29 & MAA \\
\hline
\end{tabular}




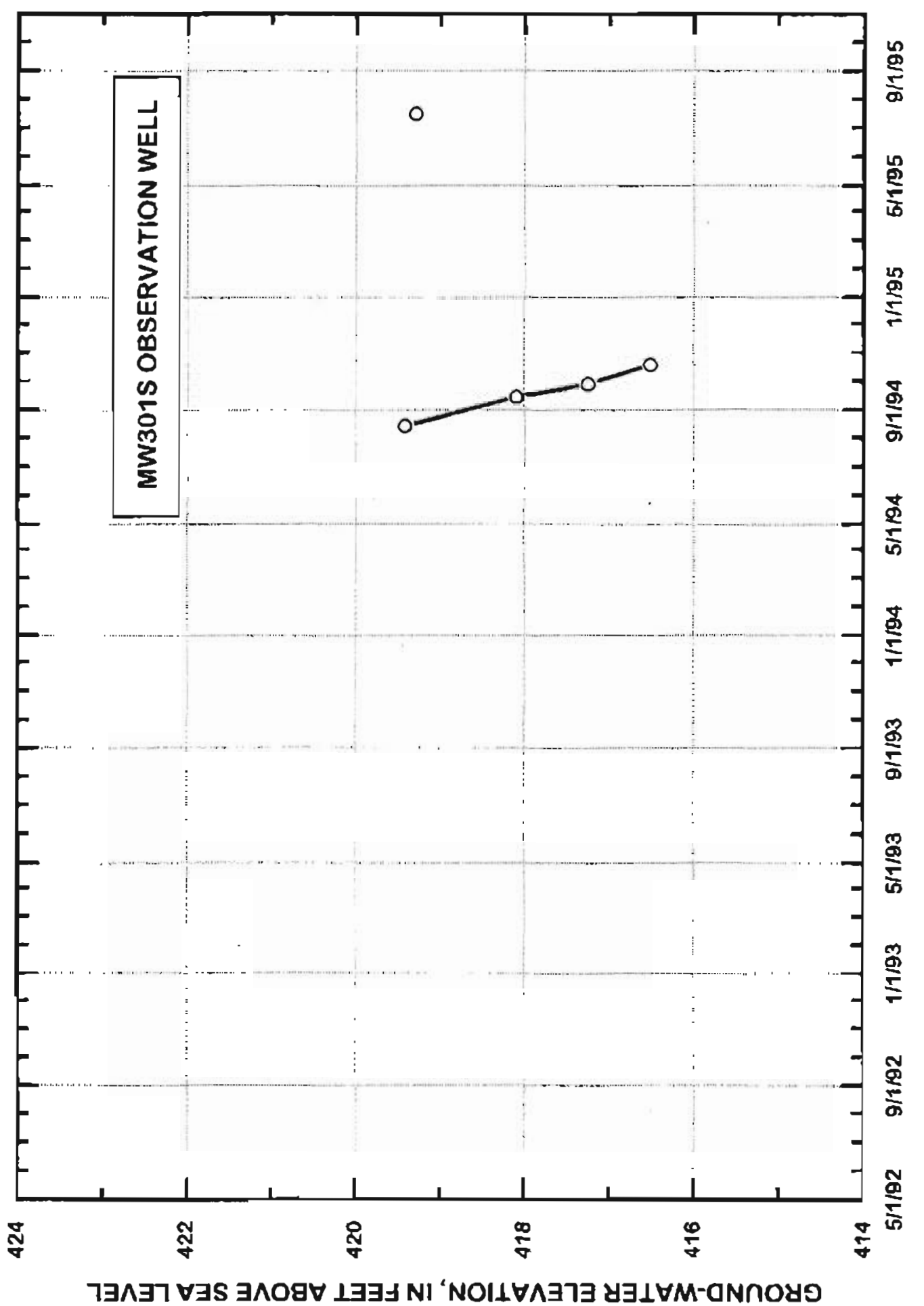


MW302D GROUND-WATER OBSERVATION WELL.

All measurements in feet Depth to bottom of well fom MP : Depth from TOC to top of Sl :

Depth from TOC to bottom of SI:

Land Surface Datum :

\begin{tabular}{c|c} 
Feet & Elevation \\
\hline 61.3 & 374.0 \\
51.3 & 384.0 \\
61.3 & 374.0 \\
& 432.2
\end{tabular}

Datum correchions, reference survey notes in site folders

$$
\begin{array}{c|c}
\text { Date } & \text { MP elevation (feet above sea level) } \\
\hline 01-23-95 & 435.32
\end{array}
$$

Site ID: Local Number:

LS, land surface

MM, mass measurement

$M P$, measuring point

NA, not available

PM, partial measutement PT, pump lest

S1, screened interval

TOC, top of casing

WS, water surface

\begin{tabular}{|c|c|c|c|c|c|c|c|}
\hline Date & Time & Method & $M P$ to WS & Error & LS to WS & WS elevation & Remarks \\
\hline $08-15-94$ & 2012 & Steel tape & 16.11 & $0 . \overline{01}$ & 12.99 & 419.21 & MM \\
\hline $09-15-94$ & 1815 & Steel tape & 15.64 & 0.01 & 12.52 & 419.68 & MM \\
\hline $09-29-94$ & 1316 & Steel tape & 18.24 & 0.01 & 15.12 & 417.08 & PM \\
\hline $10-18-94$ & 1604 & Steel tape & 18.86 & 0.01 & 15.74 & 416.46 & MM \\
\hline $02-20-95$ & 1236 & Steel tape & 19.29 & 0.01 & 16.17 & 416.03 & PT \\
\hline $02-22-95$ & 1627 & Sted tape & 19.26 & 0.01 & 16.14 & 416.06 & PT \\
\hline $05-26-95$ & 1113 & Steel tape & 17.60 & 0.01 & 14.48 & 417.72 & PM \\
\hline $07-17-95$ & 1402 & Steel tape & 16.25 & 0.01 & 13.13 & 419.07 & $\mathrm{MM}$ \\
\hline $08-16-95$ & 1550 & Stee tape & 16.69 & 0.01 & 13.57 & 418.63 & $M M$ \\
\hline $09-14-95$ & 1646 & E-tape & 15.72 & 0.02 & 12.60 & 419.60 & $M A$ \\
\hline
\end{tabular}




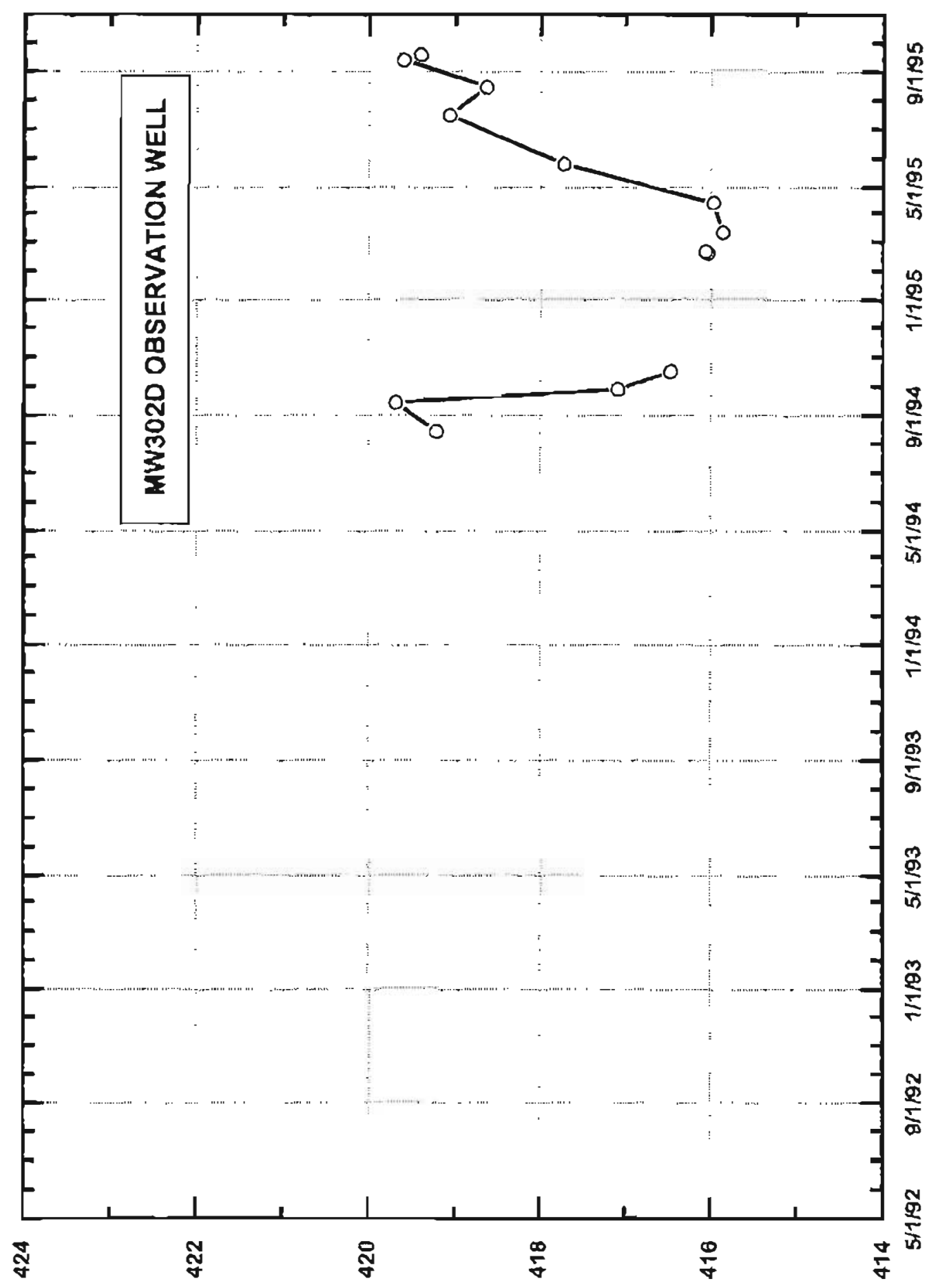

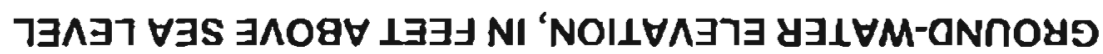


Site ID: Local Number:

\begin{tabular}{c|c} 
Feet & Elevation \\
\hline 20.7 & 414.3 \\
10.7 & 424.3 \\
20.7 & 414.3 \\
& 431.6
\end{tabular}

All measurements in feet Depth to bottom of well from MP : Depth from TOC to top of SI : Depth from TOC to bottom of Si : Land Surface Datum:

Datum corrections, reference survey notes in site folders

$$
\begin{array}{c|c}
\text { Date } & \text { MP elevation (feet above sea level) } \\
\hline 01-23-95 & 434.91
\end{array}
$$

645101147494002 FC00100106CDDC5

LS, land suface $M M$, mass measurement $M P$, measuring point NA, not available PM, partial measurement Sl, screened interval TOC, top of casing WS, water surface

\begin{tabular}{ccccccccc}
\hline Date & Time & & Method & MP to WS & Error & LS to WS & WS elevation & Remarks \\
\hline $00-15-94$ & 2019 & Steel tape & 15.70 & 0.01 & 12.39 & 419.21 & \\
$09-15-94$ & 1815 & Steet tape & 15.64 & 0.01 & 12.33 & 419.27 & MM \\
$09-29-94$ & 1321 & Steel tape & 17.81 & 0.01 & 14.50 & 417.10 & MM \\
$10-18-94$ & 1606 & Steel tape & 18.45 & 0.01 & 15.14 & 416.46 & PM & MM \\
$07-17-95$ & 1358 & Steel tape & 15.86 & 0.01 & 12.55 & 419.05 & MM & MM \\
$09-14-95$ & 1643 & E-tape & 15.31 & 0.02 & 12.00 & 419.60 & \\
\hline
\end{tabular}




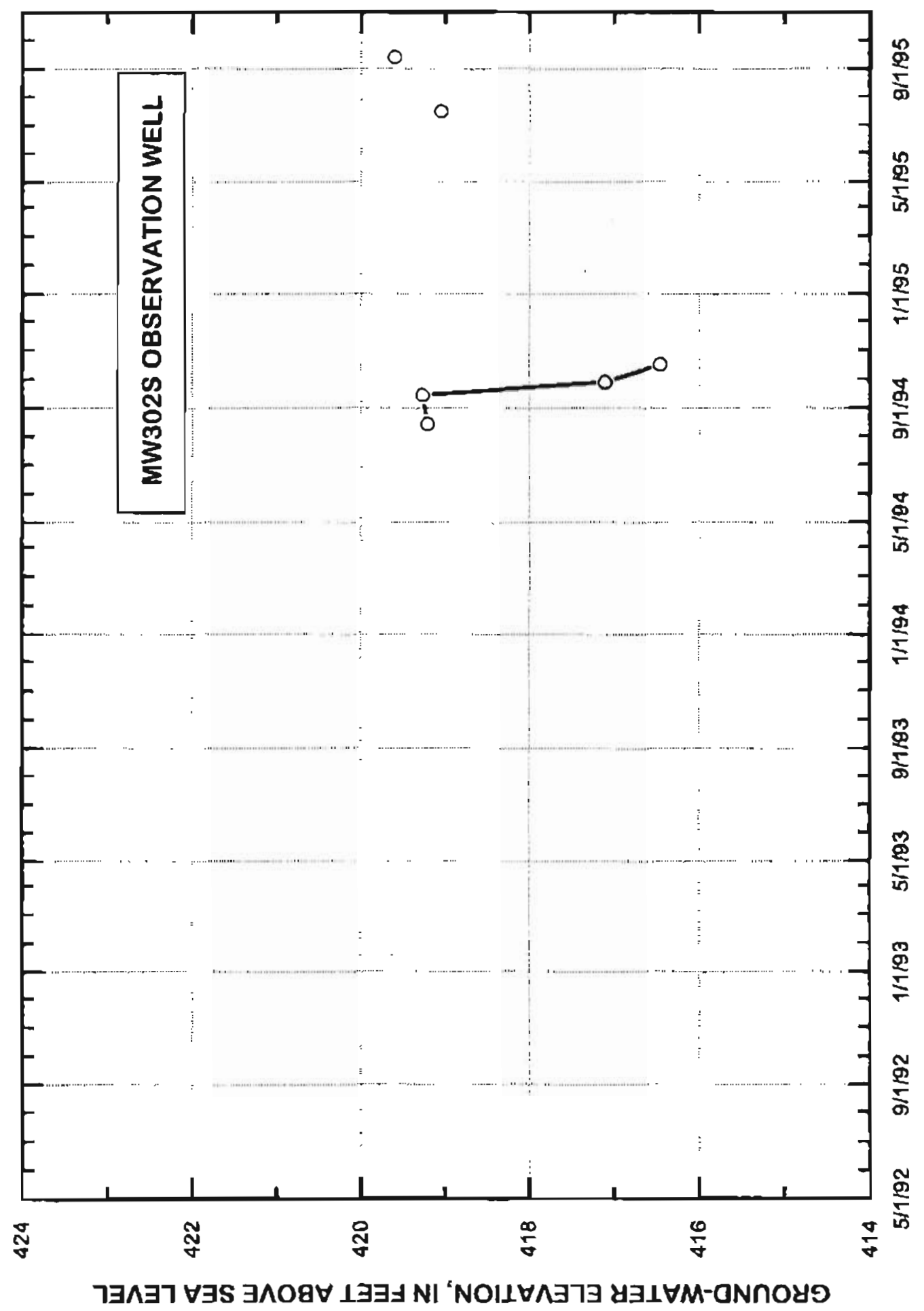


MWOOAD GROUND-WATER OBSERVATION WELL

All measurements in feet Depth to bottom of well from MP : Depth from TOC to top of SI: Depth from TOC to bottom of SI : Land Sufface Datum:

\begin{tabular}{c|c} 
Feet & Elevation \\
\hline 60.5 & 374.4 \\
50.5 & 384.4 \\
60.5 & 374.4 \\
& 431.5
\end{tabular}

Datum corrections, reterence survey notes in site folders

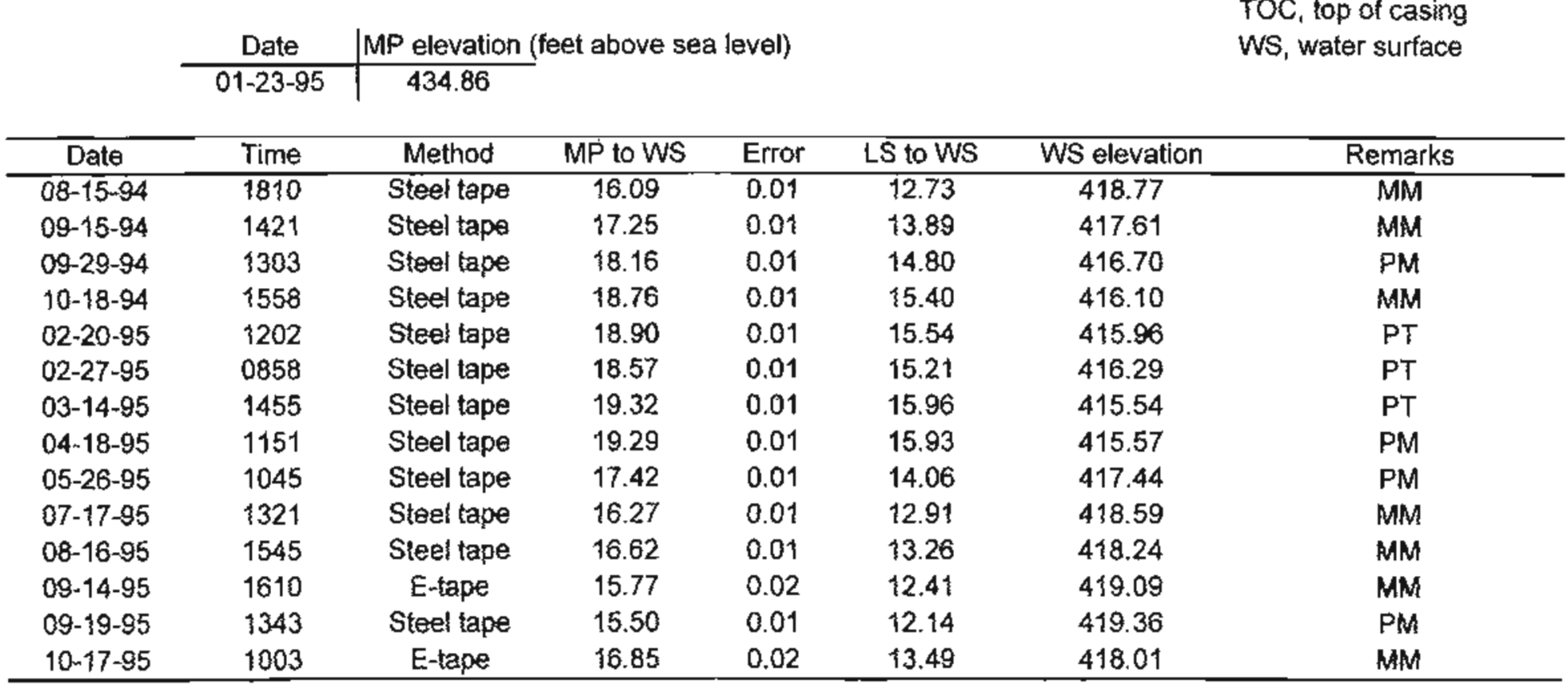

Site ID: Local Number: 645103147494002 FC00100106CDDB5

LS, land surface $M M$, mass measurement $M P$, measuring point NA, not available $P M$, partial measurement PT, pump test Si, screened interva FOC, top of casing . 


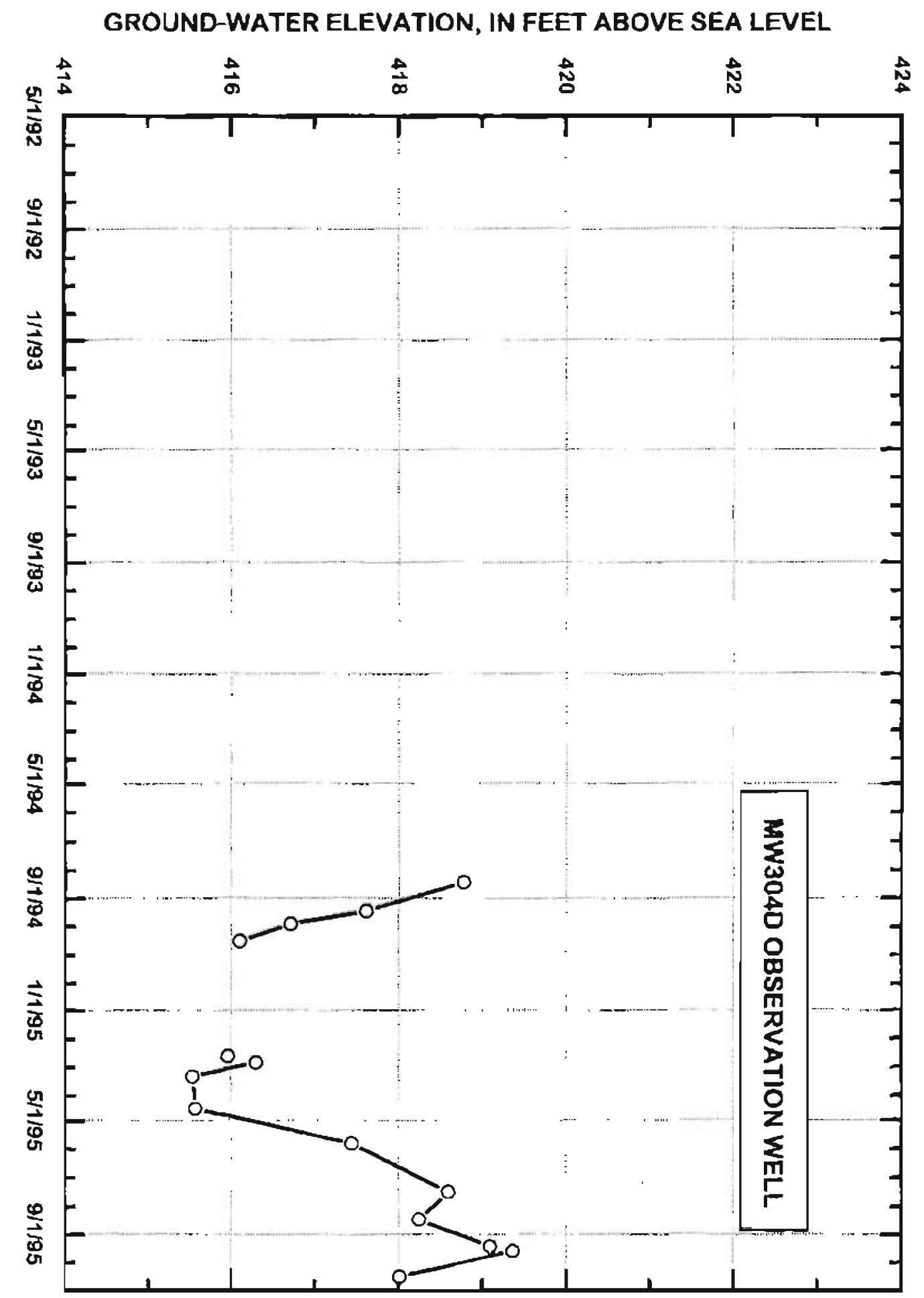


Site ID Local Number:

645103147494003 FC00100106CDDB6

All measurements in feet Depth to bottom of well from MP: Depth from 700 to top of $\mathrm{SI}$ :

\begin{tabular}{c|c} 
Feet & Elevation \\
\hline 20.0 & 414.5 \\
50.0 & 384.5 \\
60.0 & 374.5 \\
& 431.5
\end{tabular}

Land Surface Datum:

Datum corrections, reference survey notes in site folders

$$
\begin{array}{c|c}
\text { Date } & \text { MP elevation (feet above sea level) } \\
\hline 01-23-95 & 434.54
\end{array}
$$

LS, land surface $M M$, mass measurement MP, measuring point NA, not available PM, partial measurement Sl, screened interval TOC, top of casing WS, water surface

\begin{tabular}{cccccccc}
\hline Date & Time & Method & MP to WS & Error & LS to WS & WS elevation \\
\hline $08-15-94$ & 1815 & Steel tape & 15.64 & 0.01 & 12.60 & 418.90 & Remarks \\
$09-15-94$ & 1425 & Steel tape & 16.82 & 0.01 & 13.78 & 417.72 & MM \\
$09-29-94$ & 1306 & Steel tape & 17.68 & 0.01 & 14.64 & 416.86 & PM \\
$10-18-94$ & 1603 & Steel tape & 18.27 & 0.01 & 15.23 & 416.27 & 419.26 \\
$09-14-95$ & 1608 & E-tape & 15.28 & 0.02 & 12.24 & NM & MM \\
$10-17-95$ & 1015 & E-tape & 16.32 & 0.02 & 13.28 & 418.22 & MM \\
\hline
\end{tabular}




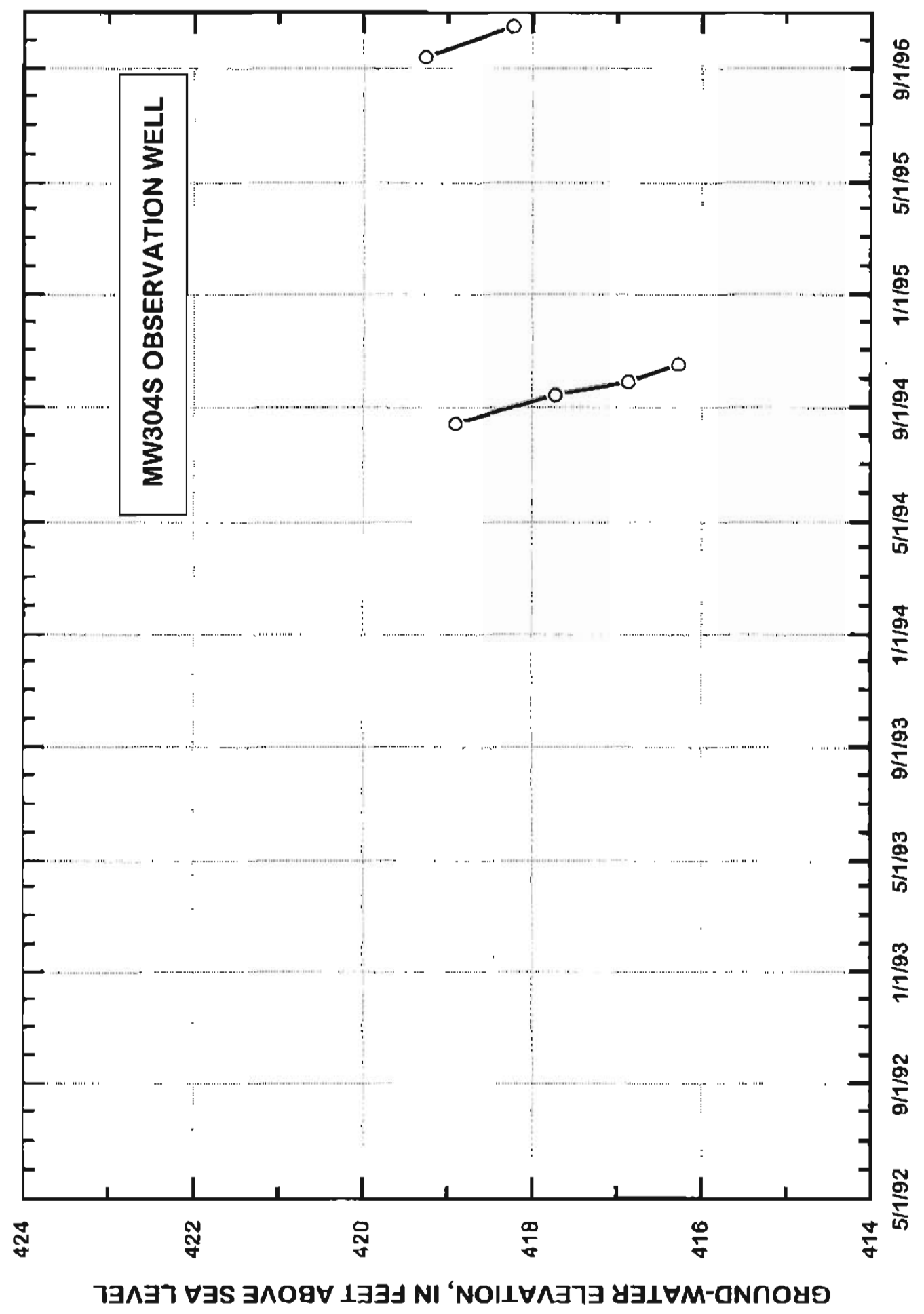


All measurements in feel

Depth to bottom of well from MP :

Depth from TOC to top of SI :

Depth from TOC to bottom of SI :

Land Surface Datum :

\begin{tabular}{c|c} 
Feel & Elevation \\
\hline 60.0 & 372.8 \\
50.0 & 382.8 \\
60.0 & 372.8 \\
& 428.9
\end{tabular}

Datum corrections, reference survey notes in site folders

\begin{tabular}{c|c} 
Date & MP elevation (feet above sea level) \\
\hline $01-23-95$ & 432.83
\end{tabular}

LS, land surface

MM, mass measurement

MP, measuring point

NA, not available

$P M$, partial measurement

PT. pump test

Sl, screened interval

TOC, top of casing

WS, water surface

\begin{tabular}{|c|c|c|c|c|c|c|c|}
\hline Date & Time & Method & $M P$ to WS & Error & LS to WS & WS elevation & Remarks \\
\hline $02-20-95$ & 1040 & Steel tape & 16.54 & 0.01 & 12.61 & $416.2 \theta$ & $\mathrm{PT}$ \\
\hline $02-27-95$ & 0932 & Steel tape & 36.70 & 0.01 & 12.77 & 416.73 & PT \\
\hline $04-45-95$ & 1157 & Steel tape & 16.22 & 0.01 & 12.29 & 416.61 & $M M$ \\
\hline $05-26-95$ & 1313 & Steel tape & 14.60 & 0.01 & 10.67 & 418.23 & PM \\
\hline $07-17-95$ & 1820 & Steel tape & 13.68 & 0.01 & 9.75 & 419.15 & $M M$ \\
\hline $08-15-95$ & 1624 & Steel tape & 13.79 & 0.01 & 9.86 & 419.04 & MM \\
\hline $09-14-95$ & 1542 & E-tape & 13.07 & 0.02 & 9.14 & 419.76 & MM \\
\hline $10-17-95$ & 1223 & E-tape & 13.78 & 0.02 & 9.85 & 419.05 & MM \\
\hline
\end{tabular}




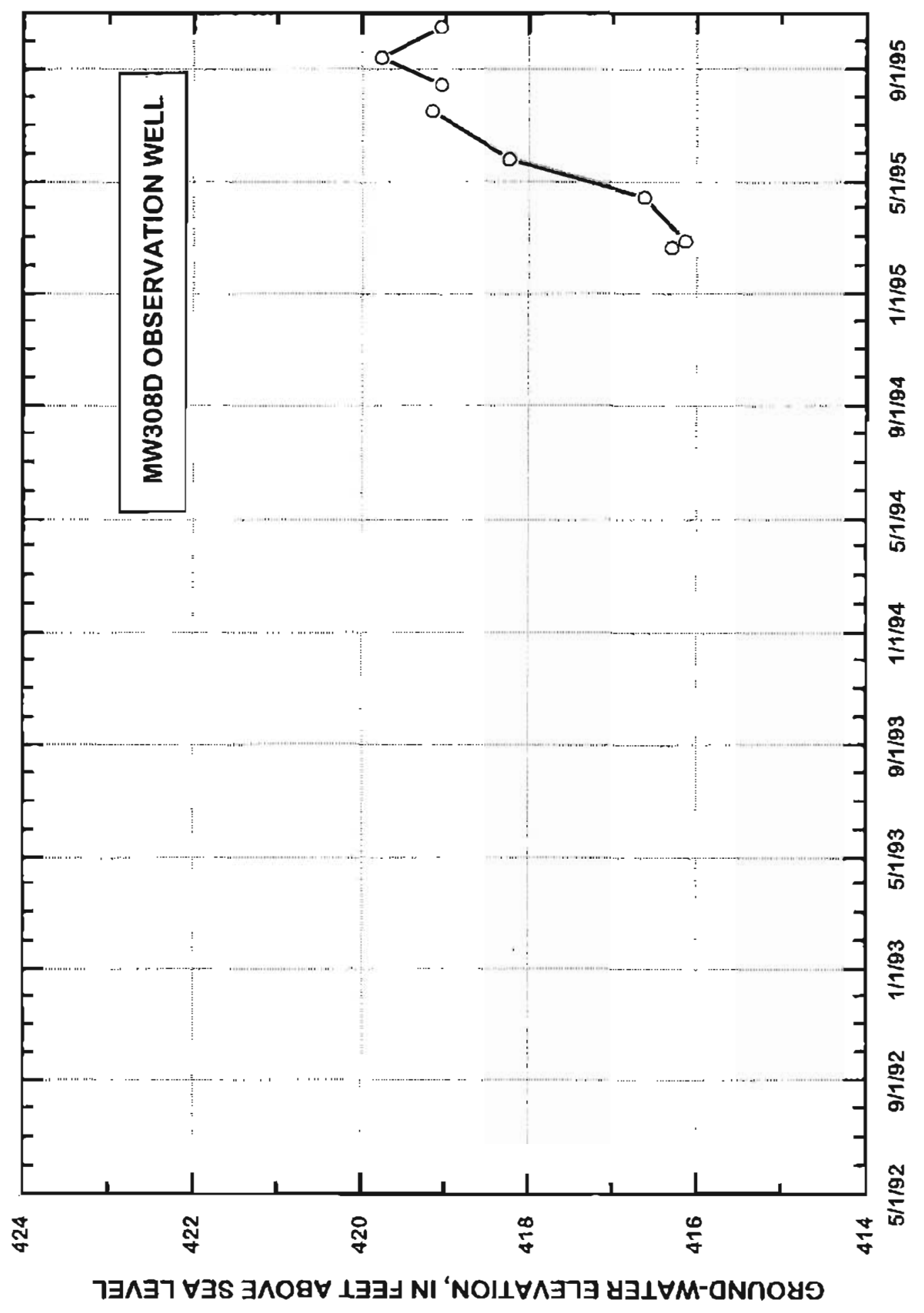



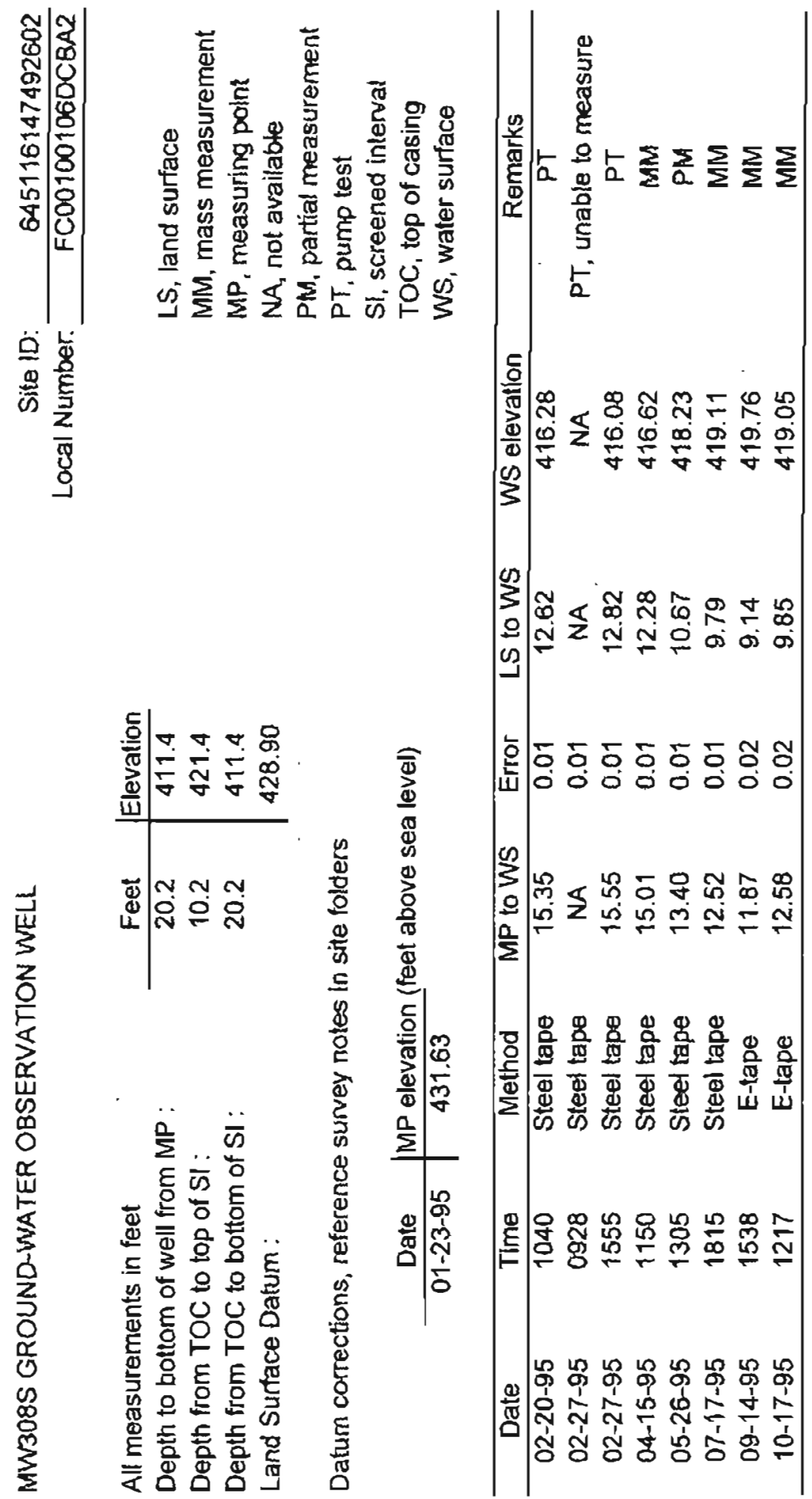


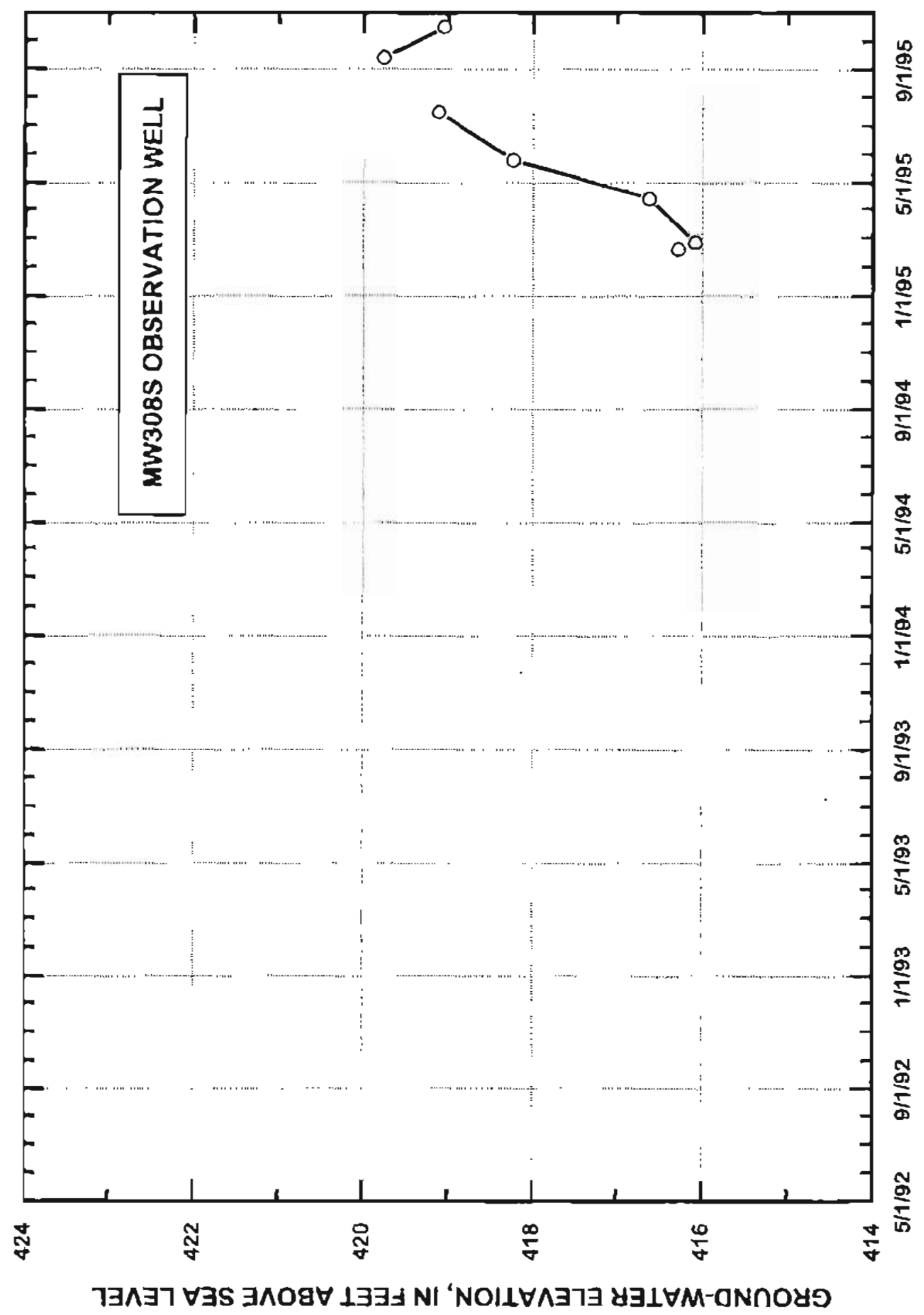


Site ID: Local Number: 645109147494001 FC00100106CDAB1

All measurements in feet

Depth to bottom of well from MP:

Depth from TOC to top of $S$ : :

Depth from TOC to bottom of S1:

\begin{tabular}{c|c} 
Feet & Elevation \\
\hline 60.0 & 376.4 \\
50.0 & 386.4 \\
60.0 & 376.4 \\
& 433.25
\end{tabular}

Datum corrections, reference survey notes in site folders

$$
\begin{array}{c|c}
\text { Date } & \text { MP elevation (feet above sea level) } \\
\hline 01-23-95 & 436.35
\end{array}
$$

LS, land surface MM, mass measurement MP, measuring point NA, not avallable PM, partial measurement Sl, screened interval TOC, top of casing WS, water surface

\begin{tabular}{|c|c|c|c|c|c|c|c|}
\hline Date & Time & Method & MP to WS & Error & LS to WS & WS elevation & Remarks \\
\hline $08-15-94$ & 1745 & Steel tape & 17.35 & 0.01 & 14.25 & 419.00 & $M M$ \\
\hline $09-15-94$ & 1410 & Steel tape & 18.20 & 0.01 & 15.10 & 418.15 & MM \\
\hline $09-29-94$ & 1245 & Steel tape & 19.02 & 0.01 & 15.92 & 417.33 & PM \\
\hline $10-18-94$ & 1526 & Steel tape & 19.65 & 0.01 & 16.55 & 416.70 & $\mathrm{MM}$ \\
\hline $07-17-95$ & 1346 & Steel tape & 17.50 & 0.01 & 14.40 & 418.85 & MM \\
\hline
\end{tabular}




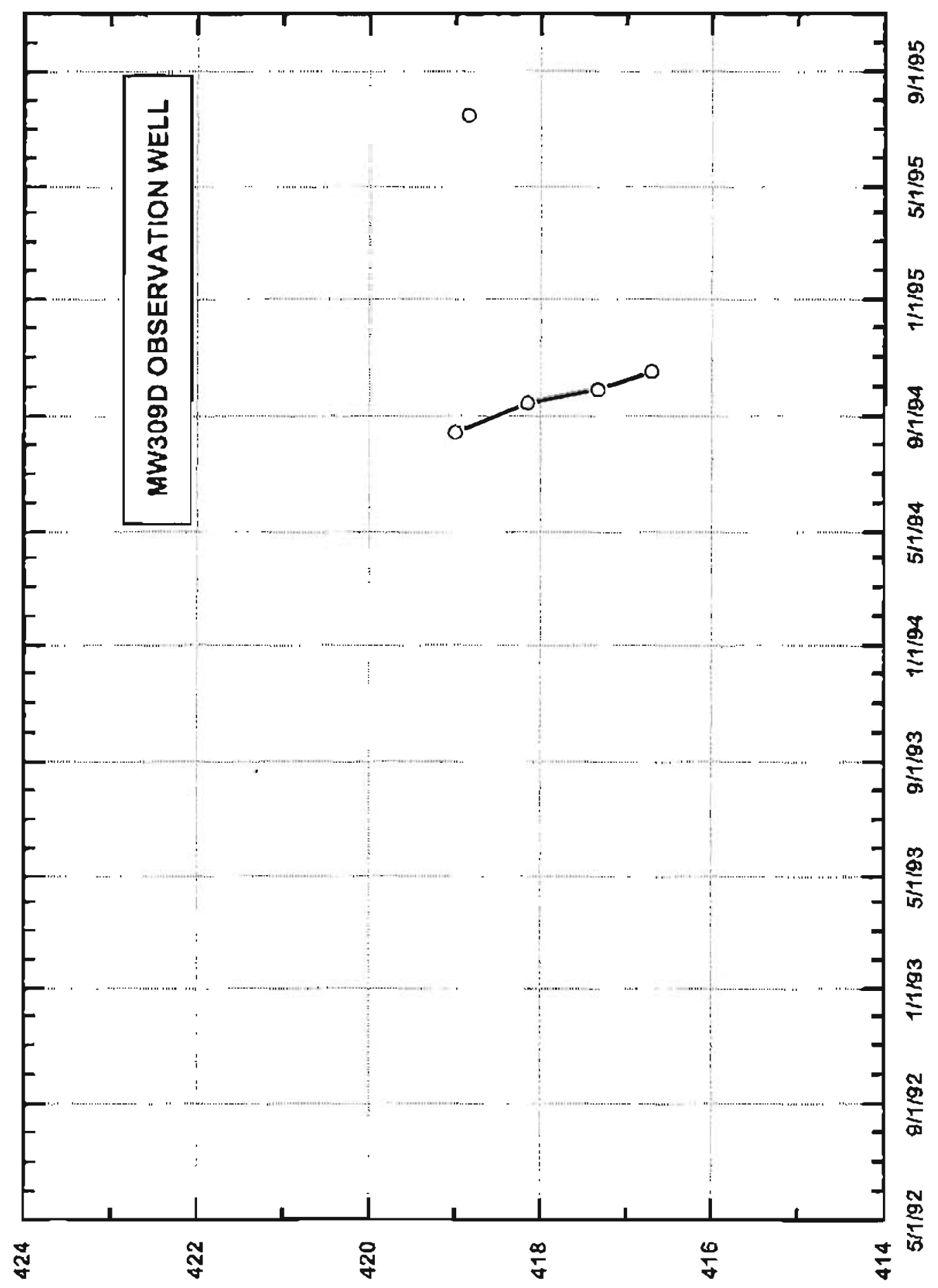

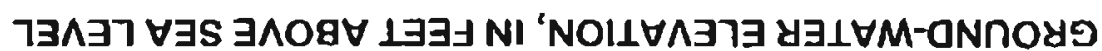


Alt measurements in feet Depth to bottom of well from MP :

Depth from TOC to top of SI :

Depth from TOC to bottom of SI:

\begin{tabular}{c|c} 
Feet & Elevation \\
\hline 21.4 & 415.5 \\
11.4 & 425.5 \\
21.4 & 415.5 \\
& 434.00
\end{tabular}

Land Surface Datum :

Datum corrections, reference survey notes in site folders

$$
\begin{array}{c|c}
\text { Dete } & \text { MP elevation (feet above sea level) } \\
\hline 01-23-95 & 436.91
\end{array}
$$

LS, land surface

MM, mass measurement

$M P$, measuring point

$N A_{\text {, not available }}$

PM, partial measurement

SI, screened interval

TOC, top of casing

WS, water surface

\begin{tabular}{cccccccc}
\hline Date & Time & Method & MP to WS & Error & LS to WS & WS elevation & Remarks \\
\hline $08-15-94$ & 1800 & Steel tape & 17.93 & 0.01 & 15.02 & 418.98 & AMA \\
$09-15-94$ & 1412 & Steel tape & 18.72 & 0.01 & 15.81 & 418.19 & MM \\
$09-29-94$ & 1252 & Steel tape & 19.54 & 0.01 & 16.63 & 417.37 & PM \\
$10-18-94$ & 1530 & Steel tape & 20.19 & 0.01 & 17.28 & 416.72 & MM \\
$07-17-95$ & 1342 & Steel tape & 18.08 & 0.01 & 15.17 & 418.83 & MM \\
\hline
\end{tabular}




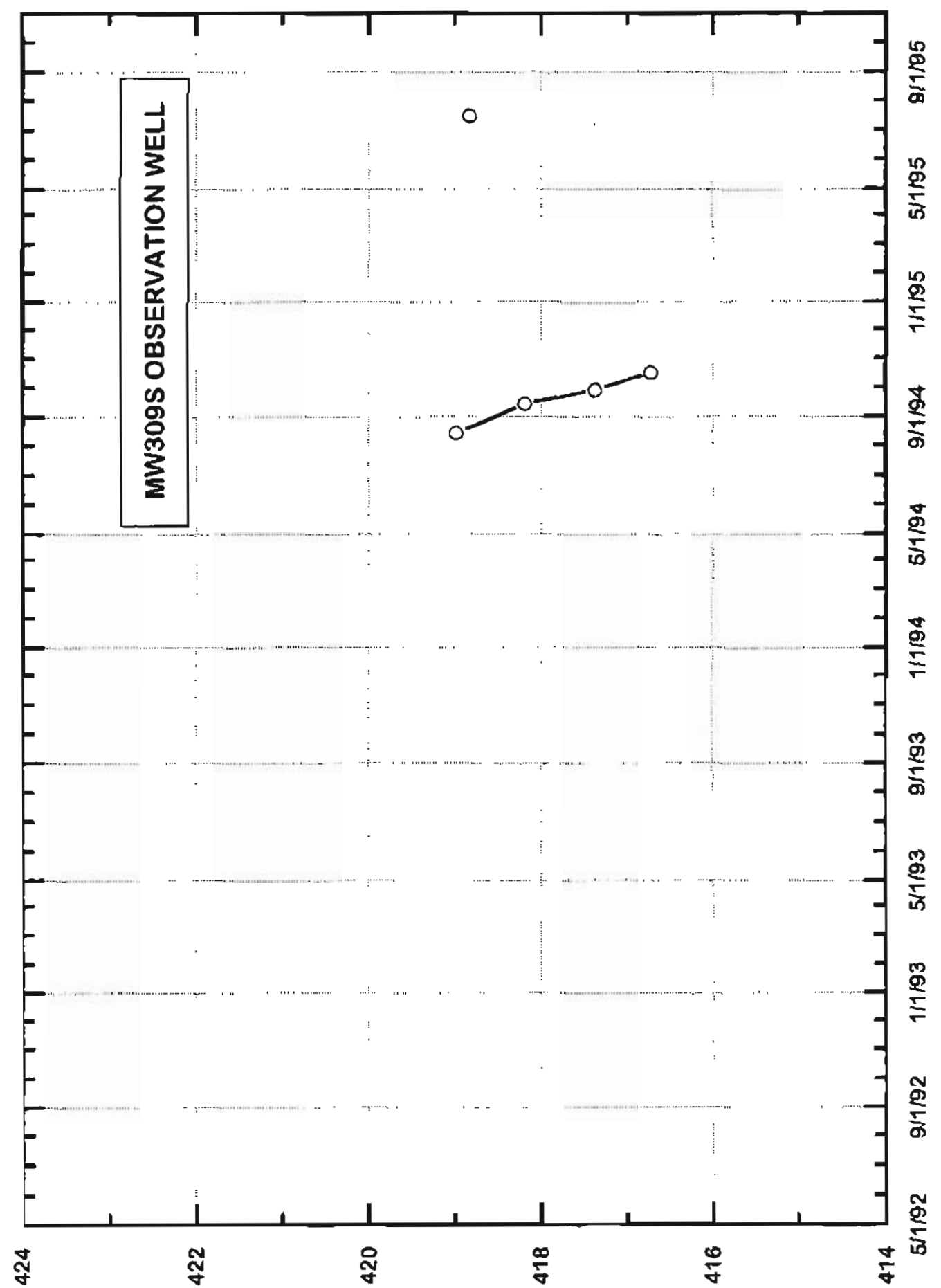

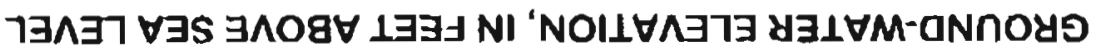


MW310 GROUND-WATER OBSERVATION WELLL

All measurements in feet

Depth to bottom of well from MP:

Depth from TOC to top of SI:

Depth from TOC to bottom of SI :

Land Surface Datum :

\begin{tabular}{c|c} 
Feet & Elevation \\
\hline 60.0 & 381.1 \\
50.0 & 391.1 \\
60.0 & 381.1 \\
& 438.00
\end{tabular}

Datum corrections, reference survey notes in site folders

\begin{tabular}{c|c} 
Date & MP elevation (feet above sea level) \\
\hline $01-23-95$ & 441.14
\end{tabular}
Site ID: Local Number:

645116147492601 FC00100106DBCB1

LS, land surface

$M M$, mass measurement

$M P$, measuring point

$N A$, not available

$P M_{1}$ partial measurement

PT, pump test

Si, screened interval

TOC, top of casing

WS, water surface

\begin{tabular}{|c|c|c|c|c|c|c|c|}
\hline Date & Time & Method & $\mathrm{MP}$ to WS & Estor & LS to WS & WS elevation & Remarks \\
\hline $08-16-94$ & 0915 & Steel tape & 21.16 & 0.01 & 18.02 & 419.98 & $M M$ \\
\hline $09-29-94$ & 1243 & Steel tape & 22.15 & 0.01 & 19.01 & 418.99 & PM \\
\hline $10-20-94$ & 1316 & Steel tape & 22.82 & 0.01 & 19.68 & 418.32 & MM \\
\hline $02-17-95$ & 1600 & Steel tape & 23.94 & 0.01 & 20.80 & 417.20 & PM \\
\hline $02-21-95$ & 1600 & Steel tape & 23.95 & 0.01 & 20.81 & 417.19 & $\mathrm{PT}^{\top}$ \\
\hline $02-26-95$ & 1720 & Steel tape & 24.91 & 0.01 & 21.77 & 416.23 & PT \\
\hline $02-27-95$ & 1010 & Steel tape & 24.96 & 0.01 & 21.82 & $4+6.18$ & $\mathrm{PT}$ \\
\hline $02-28-95$ & 1257 & Steel tape & 25.02 & 0.01 & 21.88 & 416.12 & $\mathrm{PT}$ \\
\hline 03-01-95 & 0921 & Steel tape & 25.12 & 0.01 & 21.98 & 416.02 & PT \\
\hline $03-02-95$ & 1317 & Steel tape & 24.90 & 0.01 & 21.76 & 416.24 & PT \\
\hline $03-08-95$ & 1510 & Steel tape & 25.30 & 0.01 & 22.16 & 415.84 & PT \\
\hline $03-13-95$ & 1109 & Steel tape & 25.32 & 0.01 & 22.18 & 415.82 & $\mathrm{PT}$ \\
\hline $03-20-95$ & 1444 & Steel tape & 24.22 & 0.01 & 21.08 & 416.92 & $\mathrm{MM}$ \\
\hline $04-15-95$ & 1116 & Steel tape & 23.58 & 0.01 & 20.44 & 417.56 & MM \\
\hline $06-15-95$ & 1451 & E-lape & 22.64 & 0.02 & 19.50 & 418.50 & $\mathrm{MM}$ \\
\hline $07-17-95$ & 1800 & Steel tape & 21.34 & 0.01 & 18.20 & $4 \$ 9.80$ & MM \\
\hline $08-15-95$ & 1343 & E-tape & 21.35 & 0.02 & 18.21 & 479.79 & $M A$ \\
\hline $09-14-95$ & 1531 & E-tape & 20.75 & 0.02 & 17.61 & 420.39 & $M M$ \\
\hline $10-17-95$ & 1303 & E-tape & 21.06 & 0.02 & 17.92 & 420.08 & MM \\
\hline
\end{tabular}




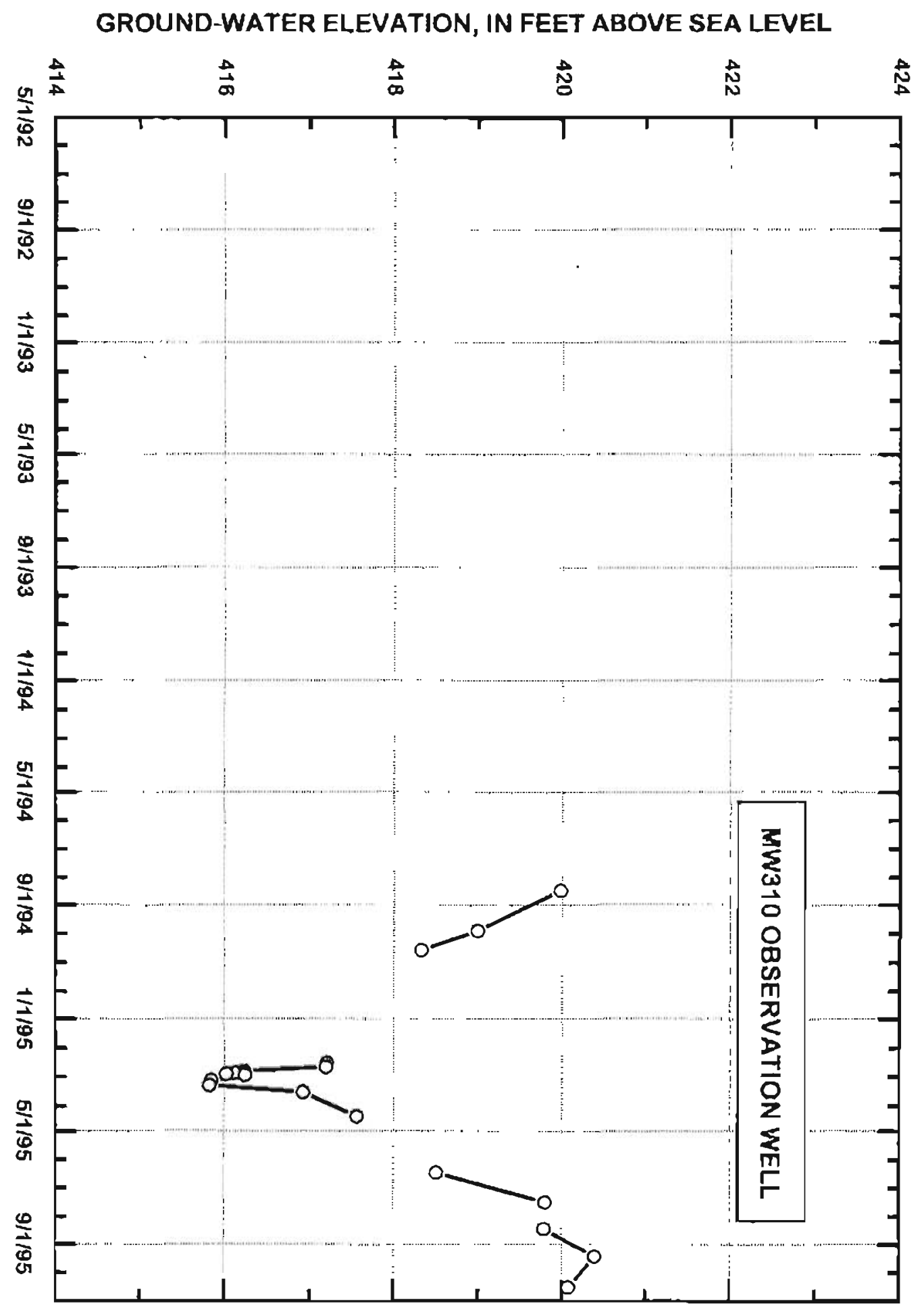


Site ID: Local Number: 645121147483504 FC00100105CBBB1

Al measurements in feet Depth to bottom of well from MP : Depth from TOC to top of SI : Depth from TOC to bottom of Si : Land Surface Datum:

\begin{tabular}{c|c} 
Feet & Elevation \\
\hline NA & NA \\
NA & NA \\
NA & NA \\
& 433.9
\end{tabular}

Datum corrections, reference survey notes in site folders

\begin{tabular}{c||c|c} 
Date & MP Elevation (feet above sea level) \\
\hline $06-23-94$ & 433.55
\end{tabular}
LS, land surface $A P$, measuring point MM, mass measurement $N A$, not available $\mathrm{PM}$, partiat measurement SI, screened interval TOC, top of casing WS, water surface

\begin{tabular}{|c|c|c|c|c|c|c|c|}
\hline Date & Time & Nethod & $M P$ to WS & Ëror & LS to WS & WS elevation & Remarks \\
\hline $11-06-92$ & 1624 & Steel tape & 17.99 & 0.01 & 18.34 & 415.56 & $M A$ \\
\hline $02-19-93$ & 1230 & Steel tape & 15.29 & 0.01 & 15.64 & 418.26 & MM \\
\hline $03-12-93$ & 1028 & Steel tape & 15.56 & 0.01 & 15.91 & 417.99 & MM \\
\hline $04-16-93$ & 1445 & Steel tape & 15.01 & 0.01 & 15.36 & 418.54 & MM \\
\hline $05-1 B-93$ & 1329 & Steel tape & 12.52 & 0.01 & 12.87 & 421.03 & MM \\
\hline $06-11-93$ & 1213 & Steel tape & 12.96 & 0.01 & 13.31 & 420.59 & MM \\
\hline $07-16-93$ & 1306 & Steol tape & 13.48 & 0.01 & 13.83 & 420.07 & MM \\
\hline $08-16-93$ & 0932 & Steed tape & 13.30 & 0.01 & 13.65 & 420.25 & $M M$ \\
\hline $06-15-94$ & 1017 & Steel tape & 14.83 & 0.01 & 15.18 & 418.72 & MM \\
\hline $07-01-94$ & 1554 & Steel tape & 13.42 & 0.01 & 13.77 & 420.13 & PM \\
\hline $07-15-94$ & 0857 & Steel tape & 12.91 & 0.01 & 13.26 & 420.64 & MM \\
\hline $08-15-94$ & 1131 & Steel tape & 13.05 & 0.01 & 13.40 & 420.50 & MM \\
\hline $09-15-94$ & 1221 & Steel tape & 13.54 & 0.01 & 13.89 & 420.01 & MM \\
\hline $04-15-95$ & 1050 & Steel tape & 15.68 & 0.01 & 16.03 & 417.87 & MM \\
\hline $05-17-95$ & 1015 & Steel tape & 14.20 & 0.01 & 14.55 & 419.35 & $M M$ \\
\hline $08-15-95$ & 1620 & E-tape & 13.41 & 0.02 & 13.76 & 420.14 & MM \\
\hline $09-14-95$ & 1137 & E-tape & 12.26 & 0.02 & 12.61 & 421.29 & MM \\
\hline $10-17-95$ & 1505 & E-tape & 12.97 & 0.02 & 13.32 & 420.58 & $M M$ \\
\hline
\end{tabular}




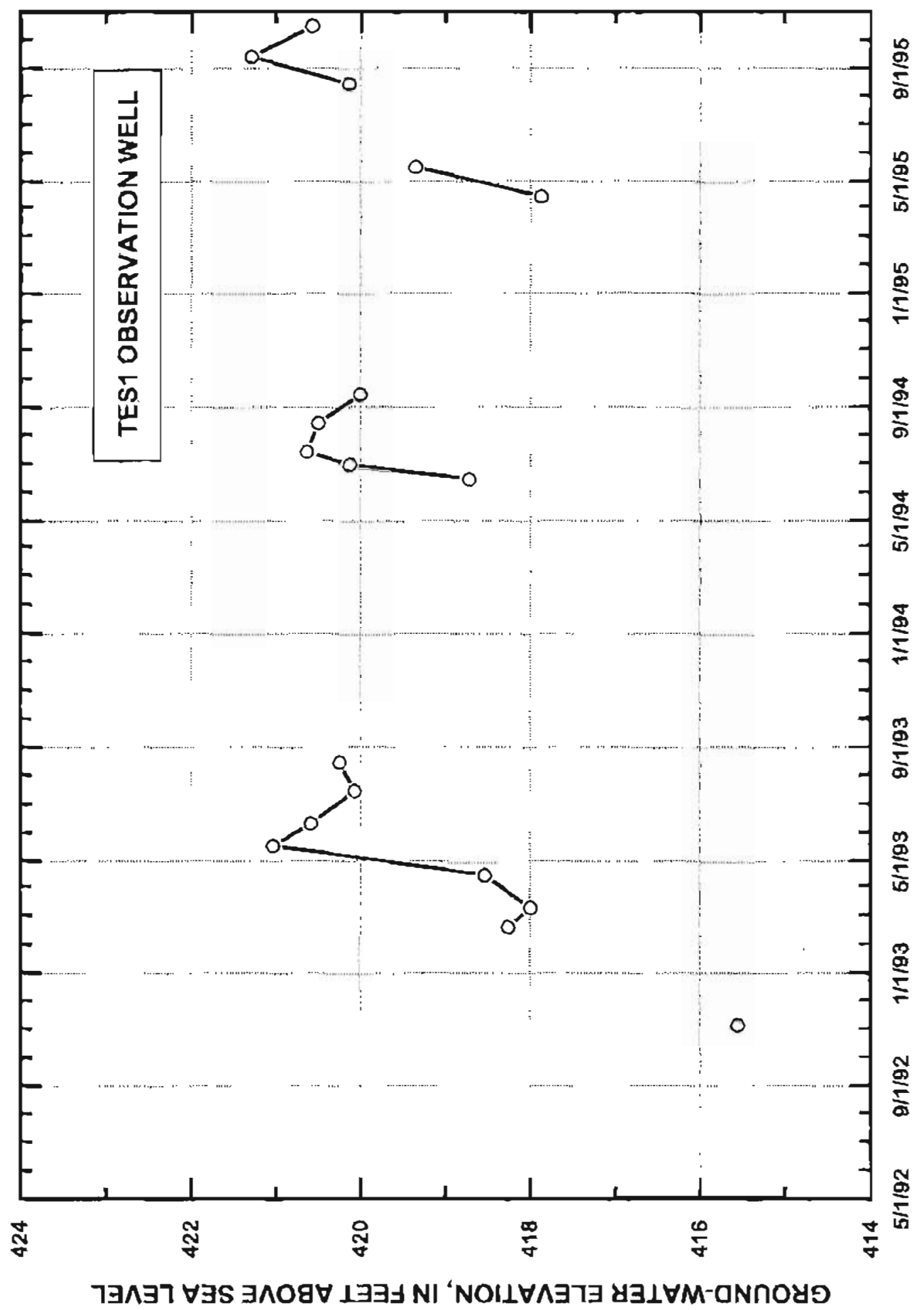


Site 10: Local Number: 645057147491601

Alt measurements in feet Depth to bottom of well from MP :

Depth from TOC to top of SI :

Depth from TOC to bottom of S! :

Land Surface Datum:

\begin{tabular}{c|c} 
Feet & Elevation \\
\hline 19.6 & 409.1 \\
16.9 & 411.8 \\
19.2 & 409.5 \\
& 426.6
\end{tabular}

Datum corrections, reference survey notes in site folders

\begin{tabular}{c|c} 
Date & MP Elevation (feet above sea level) \\
\hline $08-17-93$ & 428.71 \\
$06-23-94$ & 429.08
\end{tabular}

FC00100107ABBA1

LS, land surface

$M M$, mass measurement

MP, measuring point

$N A$, not available

PM, partial measurement

SI, screened interval

TOC. top of casing

WS, water surface

\begin{tabular}{|c|c|c|c|c|c|c|c|}
\hline Date & Tíme & Method & MP to WS & Eतror & LS to WS & WS elevation & Remarks \\
\hline $11-05-92$ & 1140 & Steel tape & $\{2.6\}$ & 0.01 & 10.50 & 416.10 & $\mathrm{MM}$ \\
\hline $12-03-92$ & 1207 & Steel tape & 11.97 & 0.01 & 9.86 & 416.74 & MM \\
\hline $12-14-92$ & 1119 & Steel tape & 11.66 & 0.01 & 9.55 & 417.05 & $\mathrm{MW}$ \\
\hline $05-18-93$ & 1025 & Steel tape & 8.39 & 0.01 & 6.28 & 420.32 & MA \\
\hline $06-03-93$ & 1140 & Stee tape & 8.77 & 0.01 & 6.56 & $4+9.94$ & $\mathrm{PM}$ \\
\hline $06-11-93$ & 1230 & Steel tape & 9.17 & 0.01 & 7.06 & 419.54 & MM \\
\hline $06-30-93$ & 1845 & E-tape & 9.09 & 0.02 & 6.98 & 419.62 & PM \\
\hline $07-16-93$ & 1324 & Steel tape & 9.44 & 0.01 & 7.33 & 419.27 & $A M$ \\
\hline $08-16-93$ & 1145 & Steel lape & 8.91 & 0.01 & 6.80 & 419.80 & $M M$ \\
\hline $09-15-93$ & 1625 & Steel tape & 9.03 & 0.01 & 6.92 & 419.68 & MM \\
\hline $10-19-93$ & 1423 & Steel tape & 9.46 & 0.01 & 7.35 & 419.25 & MM \\
\hline $11-16-93$ & 1328 & Steel tape & 10.27 & 0.01 & 8. 16 & 418.44 & $M M$ \\
\hline $12-22-93$ & 1339 & E-tape & 10.43 & 0.02 & 8.32 & 418.28 & MM \\
\hline $01-20-94$ & 1326 & E-tape & 10.95 & 0.02 & 8.84 & 417.76 & MM \\
\hline $02-24-94$ & 1432 & Steel tape & 11.35 & 0.01 & 9.24 & 417.36 & MM \\
\hline $03-16-94$ & 1330 & Steel tape & 11.38 & 0.01 & 9.27 & 417.33 & $M M$ \\
\hline $04-21-94$ & 1308 & Steel tape & 11.57 & 0.01 & 9.46 & 417.14 & MM \\
\hline $06-15-94$ & 0900 & Steel tape & 11.17 & 0.01 & 9.06 & 417.54 & MM \\
\hline $07-15-94$ & 0912 & Steel tape & 8.78 & 0.01 & 6.30 & 420.30 & MM \\
\hline
\end{tabular}


TH1 GROUND-WATER OBSERVATION WELL (Continued)

Site ID:

645057147491601

Local Number:

\begin{tabular}{|c|c|c|c|c|c|c|c|}
\hline Date & Time & Method & MP to WS & Error & LS to WS & WS elevation & Remarks \\
\hline $08-15-94$ & 1101 & Steel tape & 8.71 & 0.01 & 6.23 & 420.37 & MM \\
\hline $09-15-94$ & 1154 & Steel tape & 9.85 & 0.01 & 7.37 & 419.23 & MM \\
\hline $10-18-94$ & 1503 & Steel tape & 11.35 & 0.01 & 8.87 & 417.73 & MM \\
\hline $11-16-94$ & 1435 & Steel tape & 11.62 & 0.01 & 9.14 & 417.46 & MM \\
\hline $01-18-95$ & 1445 & Steel tape & NA & NA & NA & NA & MM, buried under snow \\
\hline $05-16-95$ & 1325 & Steel tape & 10.06 & 0.01 & 7.58 & 419.02 & MM \\
\hline $06-14-95$ & 1453 & Steel tape & 10.74 & 0.01 & 8.26 & 418.34 & MM \\
\hline $07-18-95$ & 1105 & Steel tape & 8.75 & 0.01 & 6.27 & 420.33 & $M M$ \\
\hline $08-15-95$ & $\{443$ & E-tape & 9.31 & 0.02 & 6.83 & 419.77 & MA \\
\hline $09-14-95$ & 1159 & E-tape & 8.00 & 0.02 & 5.52 & 421.08 & MM \\
\hline $10-17-95$ & 1045 & E-tape & 9.67 & 0.02 & 7.19 & 419.41 & $M M$ \\
\hline
\end{tabular}




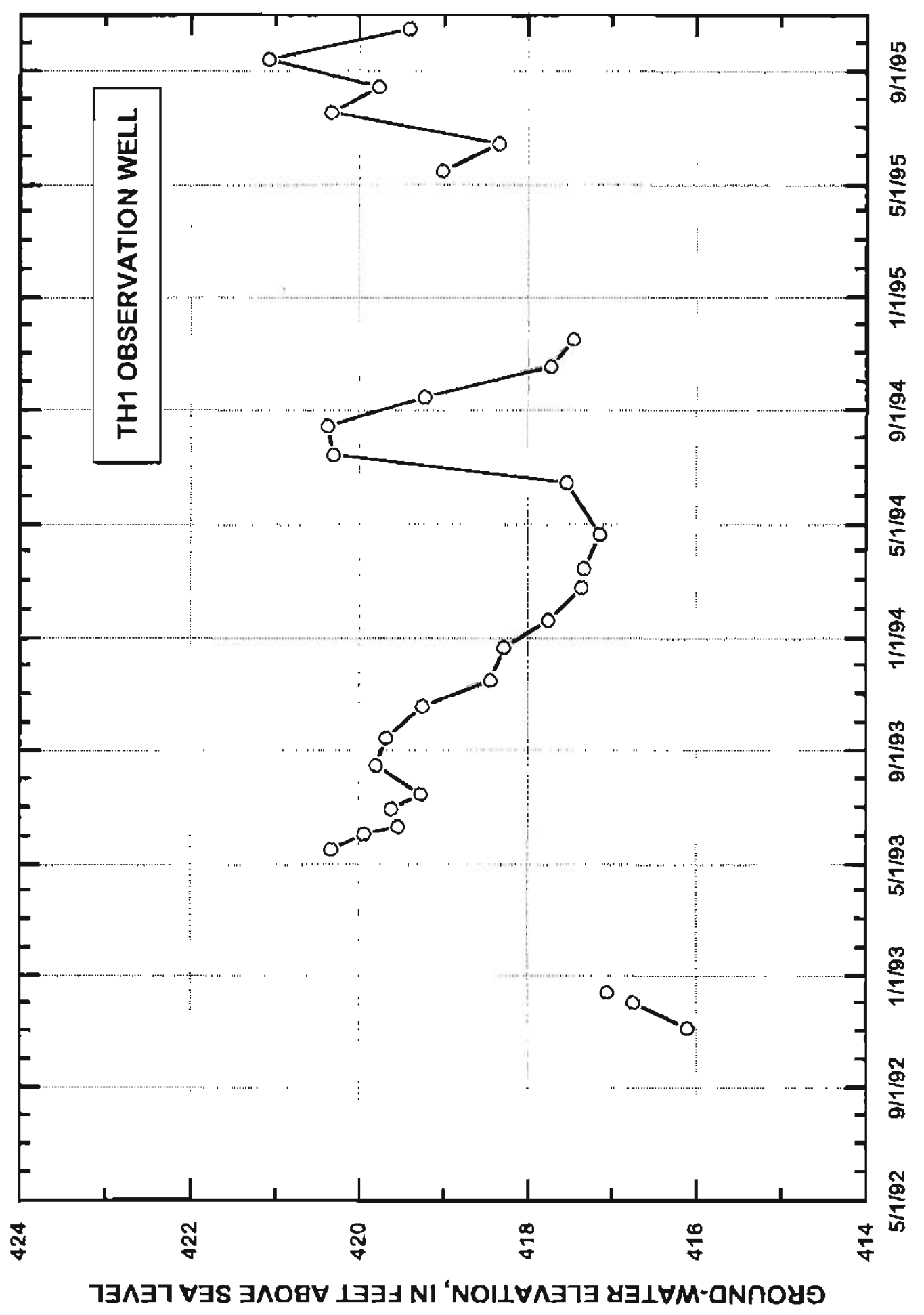


Site $\{\mathrm{D}$ : Local Number

\begin{tabular}{c|c} 
Feet & Elevation \\
\hline 22.9 & 406.2 \\
20.2 & 408.9 \\
22.4 & 406.7 \\
& 426.8
\end{tabular}

All measurements in feet Depth to bottom of well from MP : Depth from TOC to top of SI:

Depth from TOC to bottom of $\mathrm{SI}$ :

Land Surface Datum :

426.8

Dahm corractions, reference survey notes in site folders

\begin{tabular}{c|c} 
Date & MP Elevation (feel above sea level) \\
\hline $08-17-93$ & 428.79 \\
$06-23-94$ & 429.04
\end{tabular}

LS, land surface

$\mathrm{MM}$, mass measurement $M P$, measuring point

NA, not availabte

PM, partial measurement

$S I$, screened interval

TOC, top of casing

WS, water surface

\begin{tabular}{|c|c|c|c|c|c|c|c|}
\hline Date & Time & Method & MP to WS & Error & LS to WS & WS elevation & Remarks \\
\hline $11-06-92$ & 1525 & Steel tape & 12.88 & 0.01 & 10.89 & 415.91 & MM \\
\hline $12-03-92$ & 1200 & Steel tape & 11.75 & 0.01 & 9.76 & 417.04 & MM \\
\hline $12-14-92$ & 1110 & Steel tape & 11.42 & 0.01 & 9.43 & 417.37 & MM \\
\hline $05-18-93$ & 1030 & Steel tape & 8.32 & 0.01 & 6.33 & 420.47 & MM \\
\hline $06-11-93$ & 1225 & Steel tape & 9.27 & 0.01 & 7.28 & 419.52 & MA \\
\hline $06-30-93$ & 1840 & E-tape & 9.21 & 0.02 & 7.22 & 419.58 & $P M$ \\
\hline $07-16-93$ & 1321 & Steel tape & 9.50 & 0.02 & 7.51 & 419.29 & $A M A$ \\
\hline $08-16-93$ & 1140 & Steel lape & 9.00 & 0.01 & 7.01 & 419.79 & $M M$ \\
\hline $09-15-93$ & 1627 & Steel tape & 9.13 & 0.01 & 7.14 & 419.66 & MM \\
\hline $10-19-93$ & 1424 & Steel tape & 8.99 & 0.01 & 7.00 & 419.80 & MM \\
\hline $11-16-93$ & 1321 & Steel tape & 10.34 & 0.01 & 8.35 & 418.45 & MNA \\
\hline $12-22-93$ & 1410 & E-tape & 10.34 & 0.02 & 8.35 & 418.45 & MA \\
\hline $01-20-94$ & 1320 & E-tape & 10.92 & 0.02 & 8.93 & 417.87 & MM \\
\hline $02-24-94$ & 1420 & Steel tape & 11.23 & 0.01 & 9.24 & 417.56 & MM \\
\hline $03-16-94$ & 1331 & Steel tape & 11.32 & 0.01 & 9.33 & 417.47 & MM \\
\hline $04-18-94$ & 2025 & Steel tape & 11.60 & 0.01 & 9.61 & 417.19 & MM \\
\hline $04-21-94$ & 1448 & Steel tape & 12.58 & 0.01 & 10.59 & 446.21 & $M M$ \\
\hline $06-15-94$ & 0904 & Steel tape & 11.04 & 0.01 & 9.05 & 417.75 & MM \\
\hline $07-15-94$ & 0907 & Steel tape & 8.75 & 0.01 & 6.51 & 420.29 & MA \\
\hline
\end{tabular}




\section{TH2 GROUND-WATER OBSERVATION WELL (Continued)}

Site ID; Local Number: WS elevation 420.43

419.11

NA

419.13

418.39

420.44

419.80

421.14

419.42

645057147491001 FC00100107ABAB1

\begin{tabular}{|c|c|c|c|c|c|c|c|}
\hline Date & Time & Mefhod & MP to WS & Enror & LS to WS & WS elevation & Remarks \\
\hline $08-15-94$ & 1105 & Steed tape & 8.61 & 0.01 & 6.37 & 420.43 & $M M$ \\
\hline $09-15-94$ & 1158 & Steel tape & 9.93 & 0.01 & 7.69 & 419.11 & MM \\
\hline $01-18 \sim 95$ & 1446 & Steel tape & NA & 0.01 & NA & NA & MM, buried under snow \\
\hline $05-16-95$ & 1317 & Steel tape & 9.91 & 0.01 & 7.67 & 419.13 & MM \\
\hline $06-14-95$ & 1459 & Steel tape & 10.65 & 0.01 & 8.41 & 418.39 & $M M$ \\
\hline $07-18-95$ & 1111 & Steet tape & 8.60 & 0.01 & 6.36 & 420.44 & $M M$ \\
\hline $08-15-95$ & 1448 & E-tape & 9.24 & 0.02 & 7.00 & 419.80 & MM \\
\hline $09-14-95$ & 1155 & E-tape & 7.90 & 0.02 & 5.66 & 421.14 & MM \\
\hline $10-17-95$ & 1051 & E-tape & 9.62 & 0.02 & 7.38 & 419.42 & $\mathrm{MM}$ \\
\hline
\end{tabular}


GROUND-WATER ELEVATION, IN FEET ABOVE SEA LEVEL

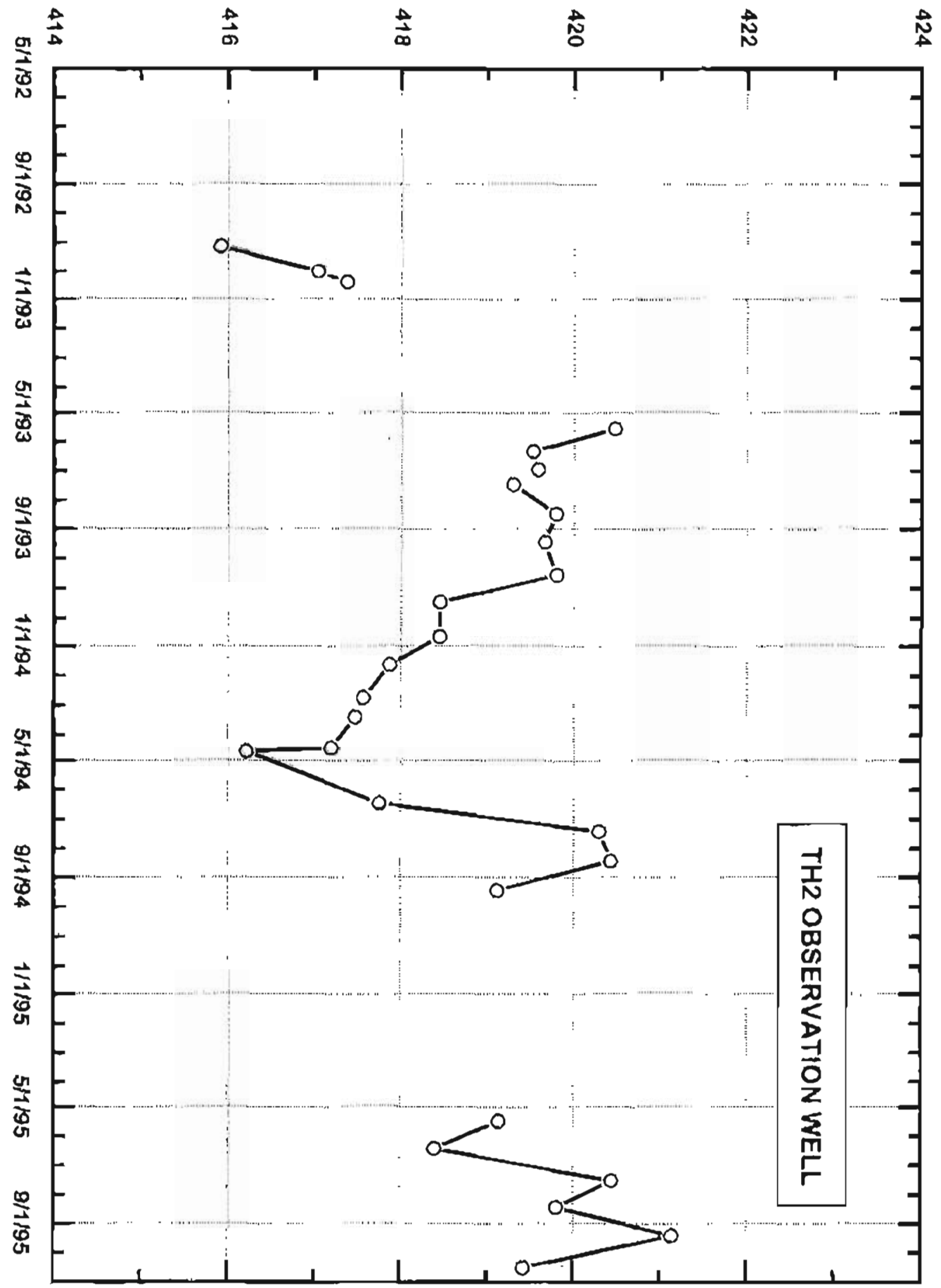


Site ID:

Local Number:

645103147491101

All measurements in feet

Depth to bottom of well from MP

Depth from TOC to top of Sl:

\begin{tabular}{c|c} 
Feet & Elevation \\
\hline 23.0 & 408.5 \\
20.3 & 411.2 \\
22.6 & 409.0 \\
& 428.53
\end{tabular}

Land Surface Datum

Datum corrections, reference survey noles in site folders

$$
\begin{array}{c|c}
\text { Date } & \text { MP Elevation (feet above sea level) } \\
\hline 08-17-93 & 431.53
\end{array}
$$

FC00100106DCDB1

LS, land surface

MM, mass measurement

MP, measuring point

NA, not available

PM, partial measurement

Sl, screened interval

TOC, top of casing

WS, water surface

\begin{tabular}{|c|c|c|c|c|c|c|c|}
\hline Date & Time & Method & MP to WS & Ërror & LS to WS & WS elevation & Remarks \\
\hline $11+06-92$ & 1500 & Steel tape & $1 \overline{5.48}$ & $\overline{0.01}$ & 12.48 & 416.05 & $M M$ \\
\hline $12-03-92$ & 1135 & Steel tape & 14.59 & 0.01 & 11.58 & 416.94 & $M M$ \\
\hline $12-14-92$ & 1221 & Steel tape & 14.28 & 0.01 & 11.28 & 417.25 & MM \\
\hline $02-20-93$ & 1630 & Steel tape & 13.68 & 0.01 & 10.68 & 417.85 & MM \\
\hline $02-25-93$ & 1242 & Steel tape & 13.73 & 0.01 & 10.73 & 417.80 & $M M$ \\
\hline $03-12-93$ & 1417 & Steel tape & 14.04 & 0.01 & 11.01 & 417.52 & MM \\
\hline $04-16-93$ & 1505 & Steet tape & 13.77 & 0.01 & 10.77 & 417.76 & $M M$ \\
\hline $08-16-93$ & 2026 & Steel tape & 11.71 & 0.01 & 8.71 & 419.82 & $M M$ \\
\hline $09-15-93$ & 1629 & Steel tape & 11.80 & 0.01 & 8.80 & 419.73 & $\mathrm{MM}$ \\
\hline $10-19-93$ & 1355 & Steel tape & 12.12 & 0.01 & 9.12 & 419.41 & MM \\
\hline $11-16-93$ & 1335 & Steel tape & 12.94 & 0.01 & 9.94 & $4 \uparrow 8.59$ & MM \\
\hline $02-24-94$ & 1503 & Steel tape & 13.88 & 0.01 & 10.88 & 417.65 & $M A$ \\
\hline $03-16-94$ & 1415 & Steel tape & 13.99 & 0.01 & 10.99 & 417.54 & $M M$ \\
\hline $04-21-94$ & 1402 & Steel tape & 14.24 & 0.01 & 11.24 & 417.29 & $M M$ \\
\hline $06-15-94$ & 1120 & Steel lape & NA & 0.01 & NA & NA & MM, blocked \\
\hline $07-15-94$ & 1120 & Steel tape & NA & 0.01 & NA & NA & MM, blocked \\
\hline $08-15-94$ & 1347 & Steel tape & 11.36 & 0.01 & 8.36 & 420.17 & $\mathrm{MM}$ \\
\hline $09-15-94$ & 1346 & Steel tape & 12.55 & 0.01 & 9.55 & 418.98 & MM \\
\hline $10-18-94$ & 1510 & Steel tape & 43.82 & 0.01 & 10.82 & 417.71 & MM \\
\hline $10-20-94$ & 1312 & Steel tape & 13.87 & 0.01 & 10.87 & $4 \uparrow 7.66$ & $\mathrm{MM}$ \\
\hline
\end{tabular}




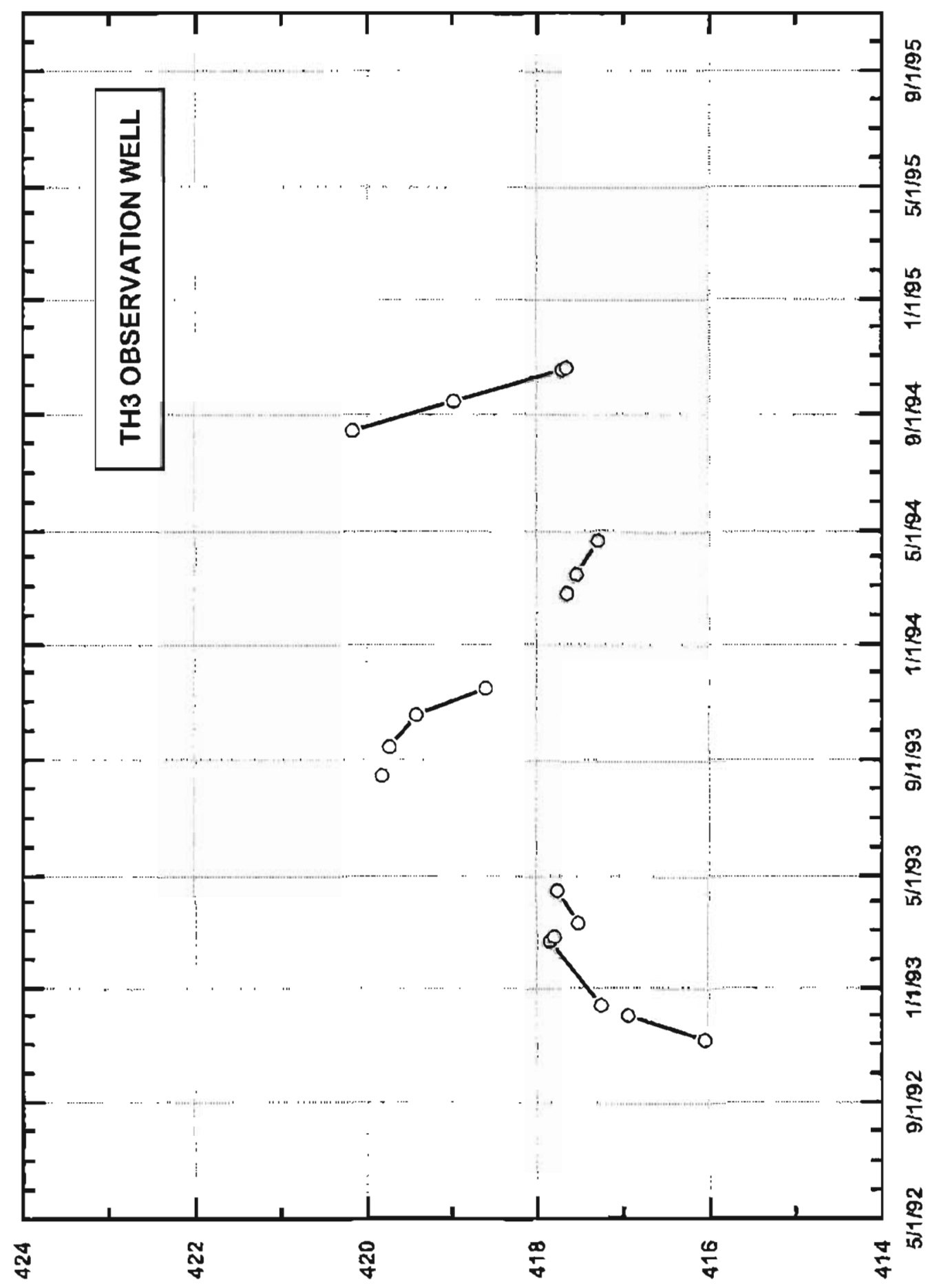

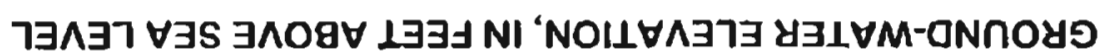


TH4 GROUND-WATER OBSERVATION WELL

All neasurements in feet

Depth to bottom of well from MP :

Depth from TOC to top of SI :

Depth from TOC to bottom of SI :

Land Surface Datum:

\begin{tabular}{c|c} 
Feet & Elevation \\
\hline 20.0 & 411.6 \\
17.3 & 414.3 \\
19.5 & 412.1 \\
& 428.9
\end{tabular}

Datum corrections, reference survey notes in site folders

$$
\begin{array}{c|c}
\text { Date } & \text { MP Elevation (feef above sea level) } \\
\hline 08-17-93 & 431.55
\end{array}
$$

Site ID:

Local Number: 645106147490401

LS, land surface

$M M$, mass measurement

$M P$, measuring point

NA, not available

$P M_{1}$ partial measurement

SI, screened interval

TOC, top of casing

WS, water surface

\begin{tabular}{|c|c|c|c|c|c|c|c|}
\hline Date & Time & Pethod & MP to WS & Eiror & LS to $\mathrm{WS}$ & WS elevation & Remarks \\
\hline $11-06-92$ & 1510 & Steel tape & 15.45 & 0.01 & 12.80 & 416.10 & MM \\
\hline $12-08-92$ & 1130 & Steel tape & 1.4 .41 & 0.01 & 11.76 & 417.14 & PM \\
\hline $12-14-92$ & 1233 & Steed tape & 14.25 & 0.01 & $\uparrow 1.60$ & 417.30 & $M M$ \\
\hline $02-25-93$ & 1255 & Steel tape & 13.62 & 0.01 & 10.97 & 417.93 & $M M$ \\
\hline $03-12-93$ & 1429 & Steel tape & 13.93 & 0.01 & 17.28 & 417.62 & $M M$ \\
\hline $04-16-93$ & 1511 & Steel tape & 13.64 & 0.01 & 10.99 & 417.91 & $M M$ \\
\hline $05-18-93$ & 1040 & Steel tape & 10.95 & 0.01 & 8.30 & 420.60 & $M M$ \\
\hline $06-11-93$ & 1250 & Steel tape & $\$ 1.60$ & 0.01 & 8.95 & 419.95 & $M M A$ \\
\hline $07-17-93$ & 1347 & Steel tape & 12.01 & 0.01 & 9.36 & 419.54 & $M M$ \\
\hline $08-16-93$ & 2021 & Steel tape & $\uparrow 1.61$ & 0.01 & 8.96 & 419.94 & MM \\
\hline $09-15-93$ & 1632 & Steel tape & 11.59 & 0.01 & B.94 & 419.96 & $M M$ \\
\hline $10-19-93$ & 1400 & Steel tape & 11.84 & 0.01 & 9.19 & 419.71 & MM \\
\hline $11-16-93$ & 1341 & Steel tape & 12.65 & 0.01 & 10.00 & 418.90 & MH \\
\hline $02-24-94$ & 1530 & Stesl tape & 13.71 & 0.01 & 11.06 & 417.84 & $\mathrm{AM}$ \\
\hline $06-15-94$ & 0910 & Steel tape & 13.45 & 0.01 & 10.80 & 418.10 & MAH \\
\hline $07-15-94$ & 1055 & Steel tape & 11.13 & 0.01 & 8.48 & 420.42 & $M M$ \\
\hline $08-15-94$ & 1345 & Steel tape & 11.27 & 0.01 & 8.62 & 420.28 & $\mathrm{MM}$ \\
\hline $09-15-94$ & 1349 & Steel tape & 12.30 & 0.01 & 9.65 & 419.25 & MAM \\
\hline $10-20-94$ & 1308 & Steel tape & $\{3.55$ & 0.01 & 10.90 & 419.00 & MA \\
\hline $08-15-95$ & 1409 & E-tape & 11.90 & 0.02 & 9.25 & 419.65 & MM \\
\hline
\end{tabular}




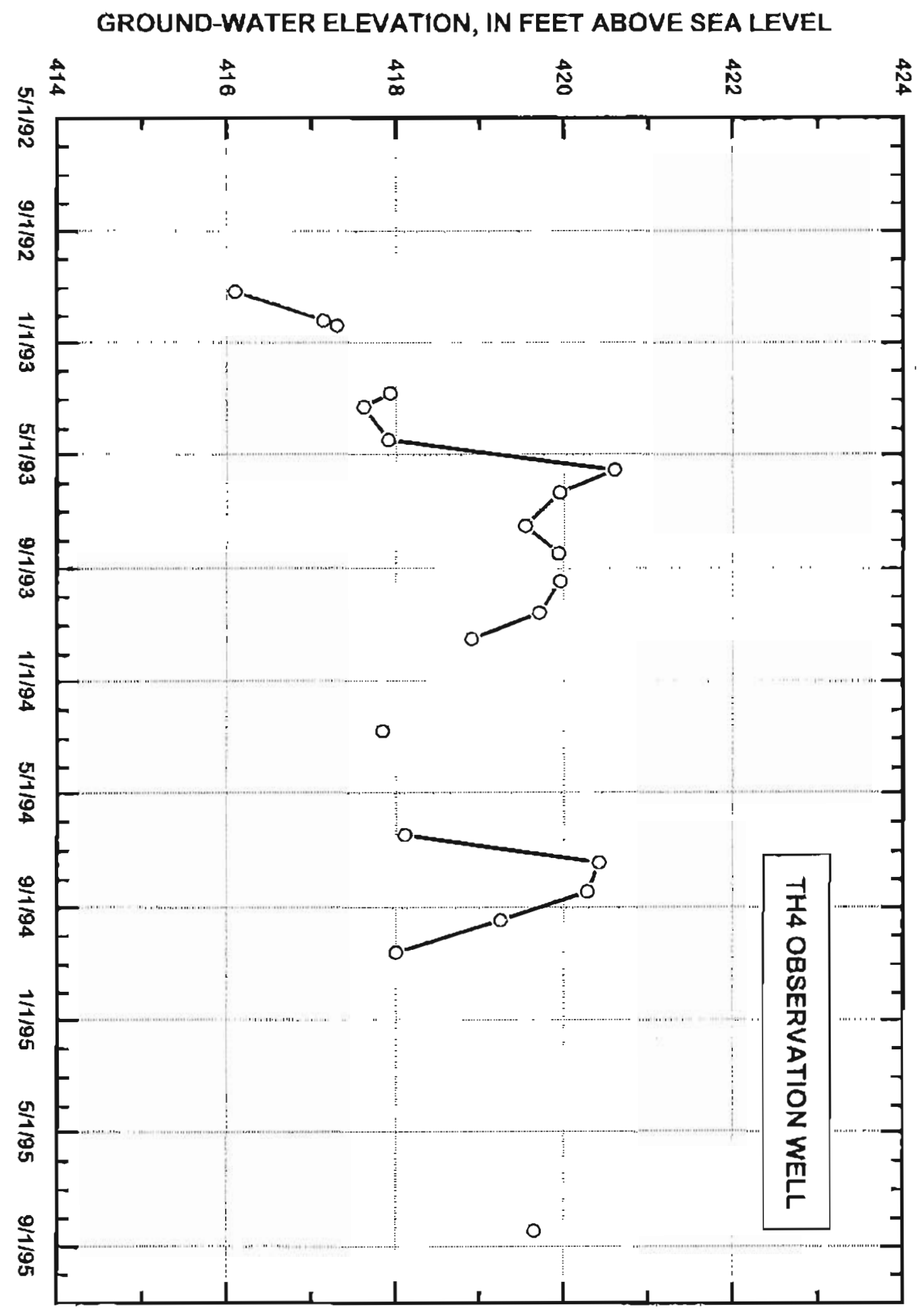


Site ID: Local Number:

\begin{tabular}{c|c} 
Feet & Elevation \\
\hline 23.0 & $404 . \overline{3}$ \\
20.3 & 407.0 \\
22.6 & 404.8 \\
& 424.0
\end{tabular}

Datum corrections, reference survey notes in site folders

\begin{tabular}{c|c} 
Date & MP Elevation (feet above sea level) \\
\hline $10-31-93$ & 427,31
\end{tabular}

All measurements in feet

Depth from TOC to top of SI :

Depth from TOC to bottom of SI:

Land Surface Datum:
Depth to bottom of well from MP :

The

\begin{tabular}{cc}
\hline Date & Time \\
\hline $11-06-92$ & 1450 \\
$12-04-92$ & 1508 \\
$12-14-92$ & 1208 \\
$02-24-93$ & 1415 \\
$02-25-93$ & 1230 \\
$03-12-93$ & 1420 \\
$04-02-93$ & 1651 \\
$04-09-93$ & 1430 \\
$05-18-93$ & 1050 \\
$06-11-93$ & 1005 \\
$07-17-93$ & 1338 \\
$08-16-93$ & 1000 \\
$10-19-93$ & 1412 \\
$10-27-93$ & 1801 \\
$10-28-93$ & 1122 \\
$11-16-93$ & 1700 \\
$12-08-93$ & 1553 \\
$12-09-93$ & 1030 \\
$12-09-93$ & 1642 \\
$12-09-93$ & 1725
\end{tabular}

\begin{tabular}{cccccc} 
Method & MP to WS & Error & LS to WS & WS elevation & Remarks \\
\hline Steel tape & 12.89 & 0.01 & 9.58 & 414.42 & MM \\
Steel tape & 11.80 & 0.01 & 8.49 & 415.51 & MM \\
Steel tape & 11.63 & 0.01 & 8.32 & 415.68 & MM \\
Steel tape & 11.58 & 0.01 & 8.27 & 415.73 & MM \\
Steel tape & 10.42 & 0.01 & 7.11 & 416.89 & MM \\
Steel tape & 10.67 & 0.01 & 7.36 & 416.64 & MM \\
Steel tape & 11.09 & 0.01 & 7.78 & 416.22 & PM \\
Steel tape & 10.94 & 0.01 & 7.63 & 416.37 & PM \\
Steel tape & 7.96 & 0.01 & 4.65 & 419.35 & MM \\
Steel tape & 8.27 & 0.01 & 4.96 & 419.04 & MM \\
Steel tape & 8.97 & 0.01 & 5.66 & 418.34 & MM \\
Steel tape & 8.45 & 0.01 & 5.14 & 418.86 & MM \\
Steel tape & 8.77 & 0.01 & 5.46 & 418.54 & MM \\
E-tape & 9.10 & 0.02 & 5.79 & 418.21 & PM \\
E-tape & 8.95 & 0.02 & 5.64 & 418.36 & MM \\
Steel tape & 11.68 & 0.01 & 8.37 & 415.63 & PM \\
E-tape & 9.85 & 0.02 & 6.54 & 417.46 & PM \\
Steel tape & 9.92 & 0.01 & 6.61 & 417.39 & PM \\
Steel tape & 9.95 & 0.01 & 6.64 & 417.36 & PM \\
Steel tape & 9.96 & 0.01 & 6.65 & 417.35 &
\end{tabular}

\section{LS, land surface}

MM, mass measurement

$M P$, measuring point

NA, not available

PM, partial measurement

SI, screened interval

TOC, top of casing

WS, water surface 


\begin{tabular}{|c|c|c|c|c|c|c|c|c|}
\hline & \multicolumn{4}{|c|}{ TH5 GROUND-WATER OBSERVATION WELL } & \multicolumn{2}{|c|}{ (Continued) } & \multirow{2}{*}{$\begin{array}{r}\text { Site } 10 \\
\text { Local Number } \\
\text { WS elevation }\end{array}$} & $\begin{array}{r}645110147492901 \\
\text { FC00100106CDAA1 }\end{array}$ \\
\hline & Date & Time & Method & MP to WS & Error & LS to WS & & Remarks \\
\hline & $12-09-93$ & 3758 & Steel tape & 9.96 & 0.01 & 6.65 & 417.35 & PM \\
\hline & $12-09-93$ & 2125 & Steel tape & 9.97 & 0.01 & 6.66 & 417.34 & PM \\
\hline & $12-22-93$ & $\{425$ & E-tape & 9.87 & 0.02 & 6.56 & 417.44 & MM \\
\hline & $01-19-94$ & 2125 & E-tape & 10.45 & 0.02 & 7.14 & 416.86 & $M M$ \\
\hline & $02-24-94$ & 1548 & Steet tape & $\uparrow 1.00$ & 0.01 & 7.69 & 416.31 & $M M$ \\
\hline & $03-16-94$ & $\{409$ & Steel tape & 11.11 & 0.01 & 7.80 & 416.20 & MM \\
\hline & $06-15-94$ & 0935 & Steel tape & 10.73 & 0.01 & 7.42 & 416.58 & MM \\
\hline & $06-21-94$ & 1703 & Steel tape & 10.21 & 0.01 & 6.90 & 417.10 & PM \\
\hline & $07-01-94$ & 1540 & Steel tape & 9.09 & 0.01 & 5.78 & 418.22 & PM \\
\hline & $07-15-94$ & 1101 & Steel tape & 8.73 & 0.01 & 5.42 & 418.58 & MM \\
\hline & $08-15-94$ & 1055 & Steel tape & 8.48 & 0.01 & 5.17 & 418.83 & MM \\
\hline & $09-15-94$ & 1403 & Steel tape & 9.18 & 0.01 & 5.87 & 418.13 & MM \\
\hline & $10-20-94$ & 1259 & Steel tape & 10.67 & 0.01 & 7.36 & 416.64 & MM \\
\hline & $02-16-95$ & 1555 & Steel tape & 11.52 & 0.01 & 8.21 & 415.79 & $\mathrm{MM}$ \\
\hline & $04-15-95$ & 1147 & Steel tape & NA & 0.01 & NA & NA & MM, slough filled with $\mathrm{H}_{2} \mathrm{O}$ \\
\hline$\xi$ & $07-17-95$ & 1835 & Steel tape & 8.77 & 0.01 & 5.46 & 418.54 & MM \\
\hline 恧 & $08-15-95$ & 1434 & E-tape & 8.90 & 0.02 & 5.59 & 418.41 & MM \\
\hline 㿼 & $09-14-95$ & 1554 & Steel tape & 8.15 & 0.01 & 4.84 & 419.16 & MM \\
\hline
\end{tabular}




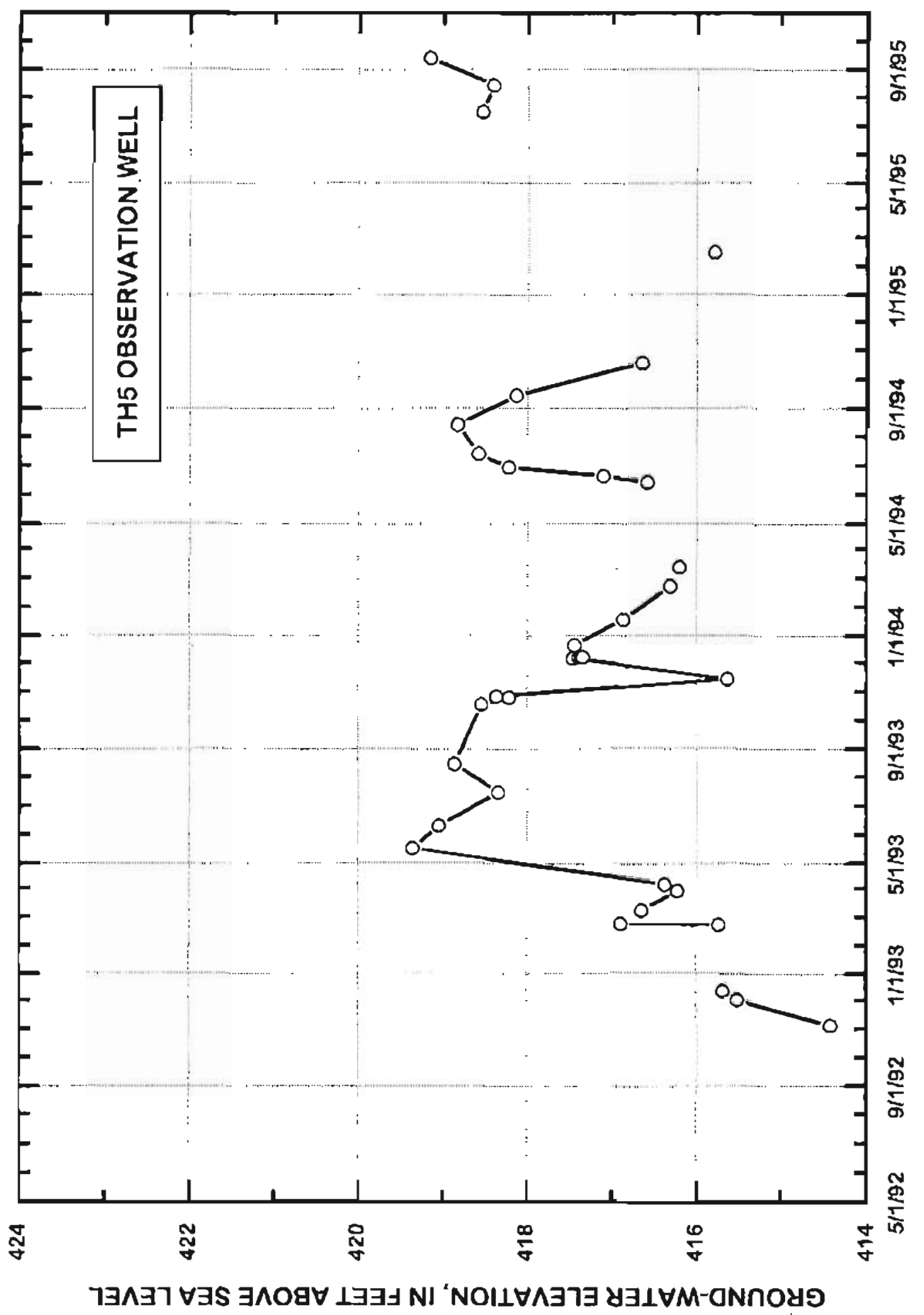


TH6 GROUND-WATER OBSERVATION WELL

All measurements in feet

Deplit to botion of well from MP :

Depth from TOC to top of $\mathrm{S1}$ :

Depth from TOC to bottom of SI:

Land Surface Datum :

\begin{tabular}{c|c} 
Feet & Elevation \\
\hline 22.0 & 412.6 \\
19.3 & 415.3 \\
21.5 & 413.1 \\
& 431.7
\end{tabular}

Datum corrections, reference survey notes in site folders

\begin{tabular}{c|c} 
Date & MP Elevation (feet above sea level) \\
\hline $02-15-93$ & 434.66 \\
$02-15-93$ & 434.58 \\
$08-18-93$ & 434.46
\end{tabular}

Site ID:

Local Number:

645107147493701 FC00100106CDAC1

LS, land surface MM, mass measurement MP, measuring point NA, not available PM, partial measurement $\mathrm{SI}_{\text {, screened interval }}$ TOC, top of casing WS, water surface

\begin{tabular}{cccccccc}
\hline Date & Time & Method & MP to WS & Error & LS to WS & WS elevation & Remarks \\
\hline $11-06-92$ & 1440 & Steel tape & 20.43 & 0.01 & 17.47 & 414.23 & MM \\
$12-04-92$ & 1430 & Steel tape & 19.31 & 0.01 & 16.35 & 415.35 & MM \\
$12-15-92$ & 1045 & Steel tape & 19.15 & 0.01 & 16.19 & 415.51 & MM
\end{tabular}

02-15-93 1430

$02-19-93 \quad 1010$

02-22-93 1609

02-24-93 0918

02-24-93 $\quad 1352$

02-24-93 1655

02-25-93 1050

02-25-93 1203

03-12-93 1230

03-17-93 1555

04-16-93 0930

05-12-93 1045

05-18-93 1515

06-11-93 1009

07-16-93

1050
Steel tape

Steel tape

Steel tape

Steel tape

Steel tape

Steel tape

Steel tape

Steel tape

Steel tape

Steel tape

Steel tape

Steel tape

Steel tape

Steel tape

Steel tape
18.14

18.13

17.98

17.92

18.12

18.22

17.99

17.83

18.12

18.43

17.74

15.50

15.38

15.80

16.54

\subsection{1}

0.01

0.01

0.01

0.01

0.01

0.01

0.01

0.01

0.01

0.01

0.01

0.01

0.01

0.01
15.18

15.24

15.09

15.03

15.23

15.33

15.10

14.94

15.23

15.54

14.85

12.61

12.49

12.91

13.65
416.52

416.46

416.61

416.67

416.47

416.37

416.60

416.76

416.47

416.16

416.85

419.09

419.21

418.79

418.05
$P M$, instalked instr. shelter MM, updated MP

MM
$P M$
$M M$
$M M$
$P M$
$M M$
$M M$
$P M$
$M M$
$P M$
$M M$
$M M$
$M M$


Site ID:

645107147493701 Local Number:

FC00100106CDAC1

\begin{tabular}{cccccccc}
\hline Date & Time & Method & MP to WS & Error & LS to WS & WS elevation & Remarks \\
\hline $08-16-93$ & 1010 & Steel tape & 15.90 & 0.01 & 13.01 & 418.69 & MM \\
$09-16-93$ & 1417 & E-tape & 16.25 & 0.02 & 13.49 & 418.21 & MM \\
$10-19-93$ & 1305 & Steel tape & 16.41 & 0.01 & 13.65 & 418.05 & MM \\
$10-27-93$ & 1749 & Steet tape & 16.73 & 0.01 & 13.97 & 417.73 & PM \\
$10-28-93$ & 1319 & Steel tape & 16.59 & 0.01 & 13.83 & 417.87 & PM \\
$11-16-93$ & 1552 & Steet tape & 17.31 & 0.01 & 14.55 & 417.15 & MM \\
$12-07-93$ & 1737 & Steel tape & 17.06 & 0.01 & 14.30 & 417.40 & PM \\
$12-08-93$ & 1545 & E-tape & 17.34 & 0.02 & 14.58 & 417.12 & PM \\
$12-09-93$ & 0944 & Steel tape & 17.40 & 0.01 & 14.64 & 417.06 & PM \\
$12-09-93$ & 2203 & Steel tape & 17.46 & 0.01 & 14.70 & 417.00 & PM \\
$12-22-93$ & 1600 & E-tape & 17.15 & 0.02 & 14.39 & 417.31 & MM \\
$01-19-94$ & 1111 & E-tape & 17.76 & 0.02 & 15.00 & 416.70 & MM \\
$02-22-94$ & 0935 & Steel tape & 18.18 & 0.01 & 15.42 & 416.28 & MM \\
$03-16-94$ & 1358 & Steel tape & 18.28 & 0.01 & 15.50 & 416.20 & MM \\
$04-21-94$ & 1355 & Steel tape & 18.32 & 0.01 & 15.56 & 416.14 & MM \\
$05-16-94$ & 2217 & Steel tape & 17.08 & 0.01 & 14.32 & 417.38 & MM \\
$06-15-94$ & 0917 & Steel tape & 17.95 & 0.01 & 15.19 & 416.51 & MM \\
$07-15-94$ & 0950 & Steel tape & 15.82 & 0.01 & 13.06 & 418.64 & MM \\
$08-15-94$ & 0932 & Steel tape & 15.56 & 0.01 & 12.80 & 418.90 & MM \\
$09-15-94$ & 1417 & Steel tape & 15.63 & 0.01 & 12.87 & 418.83 & MM \\
$10-20-94$ & 1223 & Steel tape & 18.02 & 0.01 & 15.26 & 416.44 & MM \\
\hline
\end{tabular}




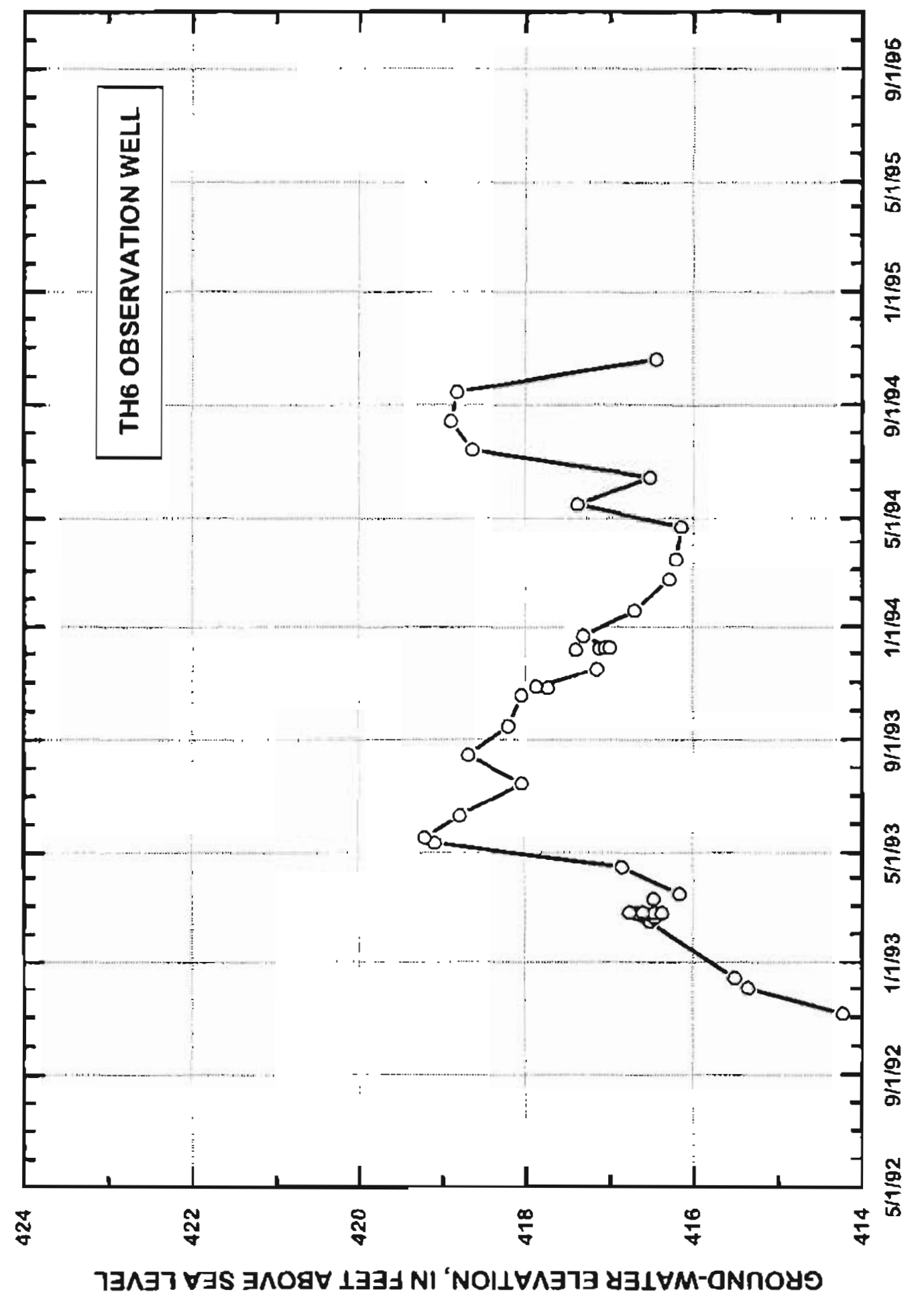


Alt measurements in feet

Depth to bottom of well from MP:

Depth from TOC to top of Si:

Depth from TOC to bottom of SI:

Land Surface Datum

\begin{tabular}{c|c} 
Feet & Elevation \\
\hline 22.9 & 409.6 \\
20.2 & 412.3 \\
22.5 & 410.1 \\
& 428.4
\end{tabular}

Datum corrections, reference survey notes in site folders

\begin{tabular}{c|c} 
Date & MP Elevation (feet above sea level) \\
\hline $07-08-93$ & 432.56 \\
$04-21-95$ & 433.19
\end{tabular}

FC00100106DBCD1

LS, land surface

MM, mass measuremen!

MP, measuring point

NA, not available

$P M$, partial measurement

PT, pump tes!

$\mathrm{SI}$, screened interval

TOC, top of casing

WS, water surface

\begin{tabular}{|c|c|c|c|c|c|c|c|}
\hline Date & Time & Method & MP to WS & EITOr & LS to WS & WS elevation & Remarks \\
\hline $10-16-92$ & 1440 & Steel tape & 18.25 & 0.01 & 14.09 & 414.31 & $P M$ \\
\hline $11-06-92$ & 1605 & Steel tape & 17.52 & 0.01 & 13.36 & 415.04 & MM \\
\hline $12-03-92$ & 1020 & Steet tape & 16.51 & 0.01 & 12.35 & 416.05 & $M M$ \\
\hline $12-15-92$ & 1100 & Steel tape & 16.21 & 0.01 & 12.05 & 416.35 & $M M$ \\
\hline $02-20-93$ & 1755 & Steel tape & 15.18 & 0.01 & 11.02 & 417.38 & $\mathrm{MM}$ \\
\hline $02-26-93$ & 1600 & Steel Tape & 15.05 & 0.01 & 10.89 & 417.51 & PM \\
\hline $03-12-93$ & 1020 & Steel tape & 15.33 & 0.01 & 11.17 & 417.23 & MM \\
\hline $04-16-93$ & 1415 & Steel tape & 14.20 & 0.01 & 10.04 & 418.36 & $M M$ \\
\hline $05-18-93$ & 1300 & Steel tape & 12.51 & 0.01 & 8.35 & 420.05 & MM \\
\hline $06-11-93$ & 1120 & Steel tape & 12.82 & 0.01 & 8.66 & 419.74 & MM \\
\hline $07-17-93$ & 1406 & Steel tape & 13.38 & 0.01 & 9.22 & 419.18 & MM \\
\hline $08-16-93$ & 1948 & Steel tape & 13.08 & 0.01 & 8.92 & 419.48 & $M M$ \\
\hline $10-19-93$ & 1516 & Steel tape & 13.03 & 0.01 & 8.87 & 419.53 & $M M$ \\
\hline $11-16-93$ & 1635 & Steel tape & 13.90 & 0.01 & 9.74 & $41 B .66$ & MM \\
\hline $12-07-93$ & 1535 & Steel tape & 14.17 & 0.01 & 10.01 & 418.39 & PM \\
\hline $12-08-93$ & 1612 & E-tape & 14.20 & 0.02 & 10.04 & 418.36 & PM \\
\hline $12-09-93$ & 1015 & Steel tape & 14.24 & 0.01 & 10.08 & 418.32 & $\mathrm{PM}$ \\
\hline $12-09-93$ & 2047 & Steel tape & 14.43 & 0.01 & 10.27 & 418.13 & $\mathrm{PM}$ \\
\hline $12-22-93$ & 1228 & E-tape & 14.38 & 0.02 & 10.22 & 418.18 & MM \\
\hline
\end{tabular}




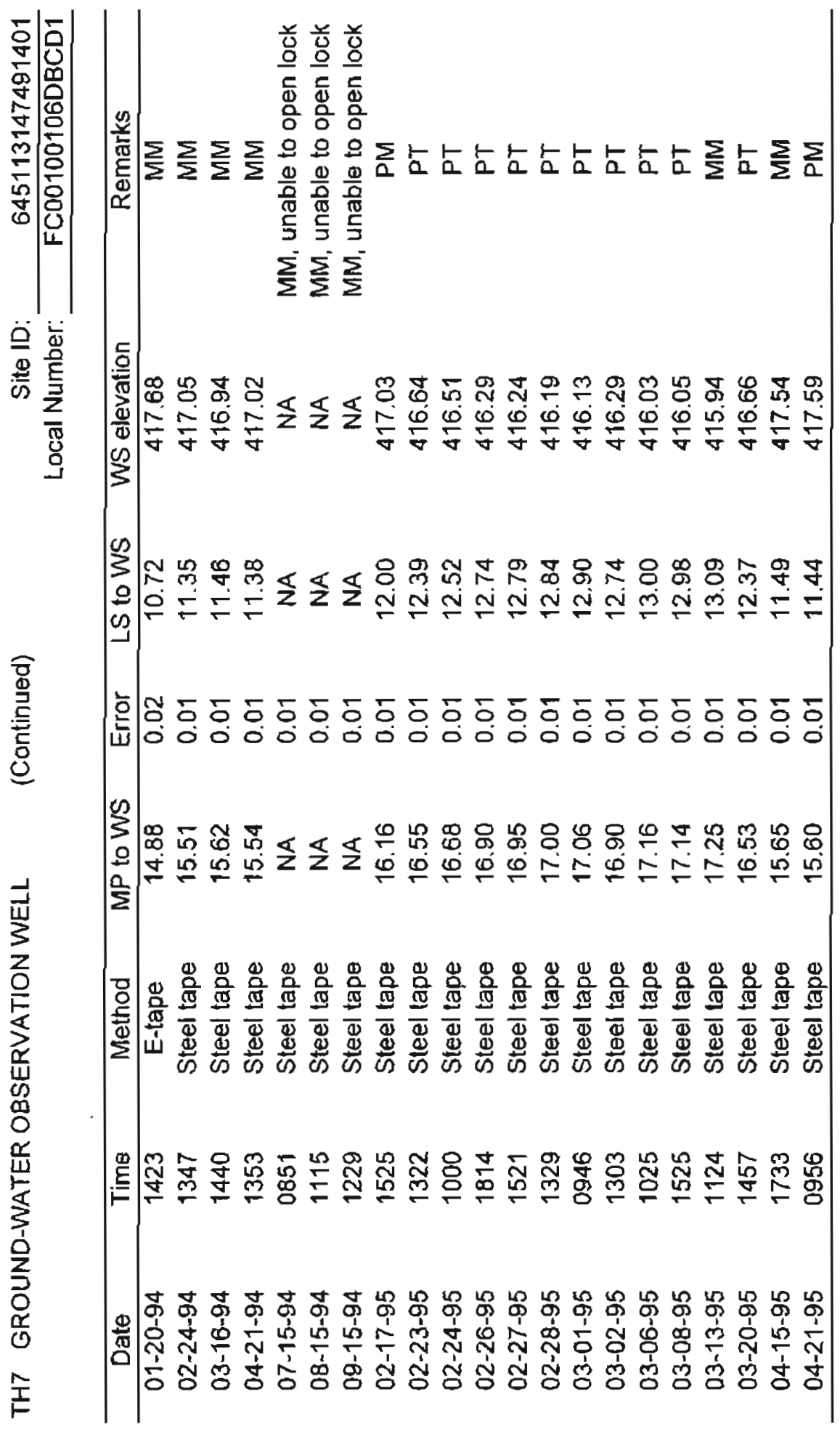




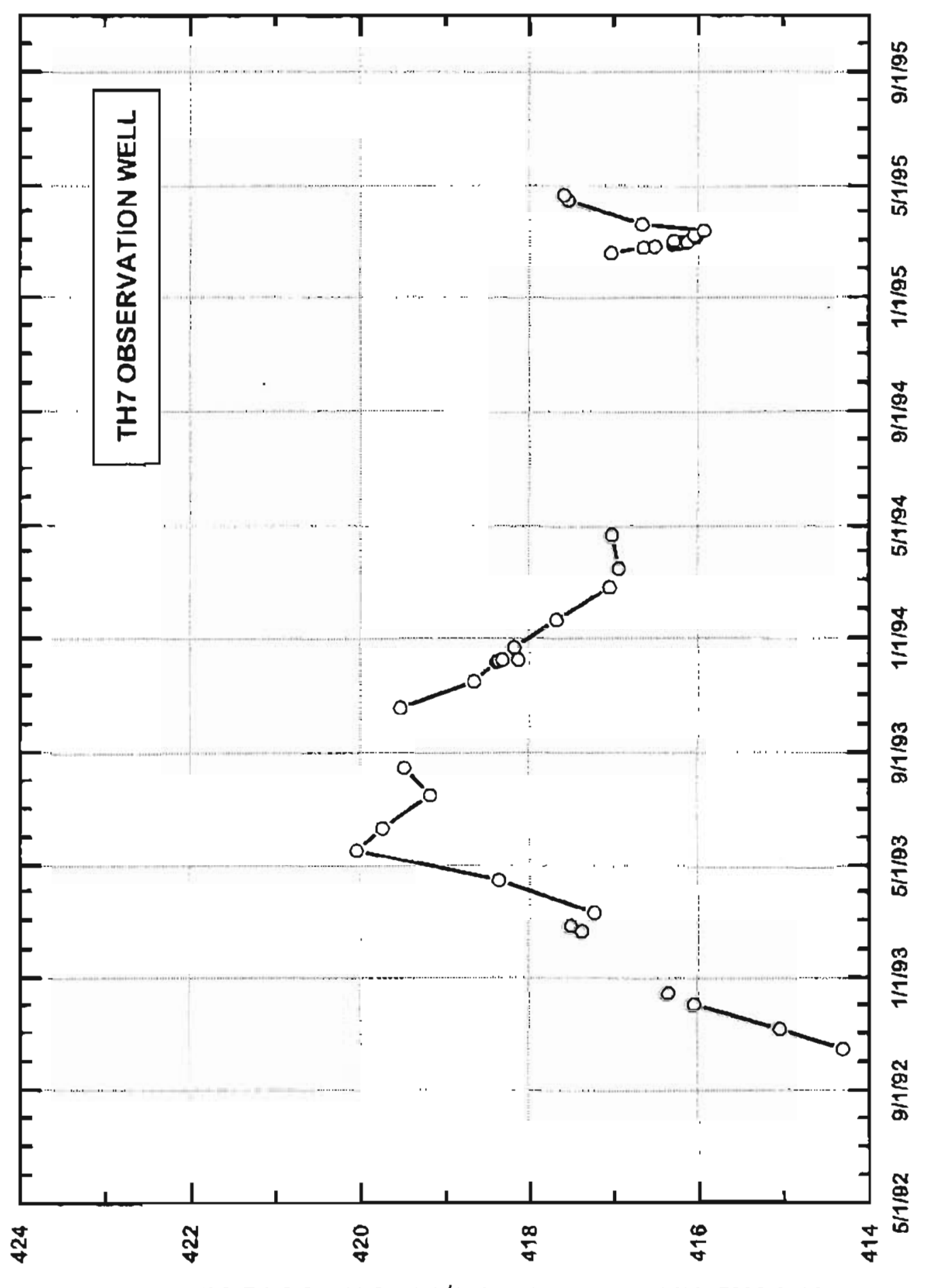

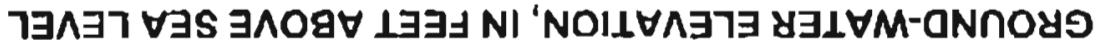


Site ID: Local Number: 645112147490201

All measurements in feet Depth to bottom of well from MP

Depth from TOC to top of SI :

Depth from TOC to bottom of SI :

\begin{tabular}{c|c} 
Feet & Elevation \\
\hline 20.8 & 412.9 \\
18.1 & 415.6 \\
20.4 & 413.4 \\
& 429.9
\end{tabular}

Datum corrections, reference survey notes in site folders

$$
\begin{array}{c|c}
\text { Date } & \text { MP Elevation (feet above sea level) } \\
\hline 07-08-93 & 433.75
\end{array}
$$

FC00100106DBDD1

LS, land surface

MM, mass measurement

$M P$, measuring point

NA, not available

PM, partial measurement

SI, screened interval

TOC, top of casing

WS, water surface

\begin{tabular}{|c|c|c|c|c|c|c|c|}
\hline Date & Time & Method & MP to WS & Error & LS to WS & WS elevation & Remarks \\
\hline $10-16-92$ & 1541 & Steet tape & 19.60 & 0.01 & 15.75 & $4 \nmid 4.15$ & PMA \\
\hline $41-06-92$ & 1600 & Steel tape & 18.65 & 0.01 & 14.80 & 415.10 & $M M$ \\
\hline $12-03-92$ & 1040 & Steel tape & 17.61 & 0.01 & 13.76 & 416.14 & $M M$ \\
\hline $12-15-92$ & 1115 & Steel tape & $\uparrow 7.24$ & 0.01 & 13.39 & 416.51 & $M M$ \\
\hline $02-20-93$ & 1745 & Steel tape & 16.13 & 0.01 & 12.28 & 417.62 & MM \\
\hline $02-26-93$ & 1620 & Steel tape & 16.05 & 0.01 & 12,20 & 417.70 & PNA \\
\hline $03-12-93$ & 1030 & Steel tape & 16.33 & 0.04 & 12.48 & 417.42 & MM \\
\hline $04-16-93$ & 1425 & Steel tape & 14.94 & 0.01 & 11.09 & 418.81 & $M M$ \\
\hline $05-18-93$ & 1313 & Steel tape & 13.48 & 0.01 & 9.63 & 420.27 & MM \\
\hline $06-11-93$ & 1127 & Steel tape & 13.77 & 0.01 & 9,92 & 419.98 & MM \\
\hline $07-17-93$ & 1413 & Steel tape & 14.33 & 0.01 & 10.48 & 419.42 & MM \\
\hline $08-\uparrow 6-93$ & 1954 & Steel tape & 14.08 & 0.01 & 10.23 & 419.67 & MM \\
\hline $10-19-93$ & 1513 & Steel tape & $\uparrow 4.01$ & 0.01 & 10.16 & 419.74 & MM \\
\hline $11-16-93$ & 1640 & Steel tape & 14.78 & 0.01 & 10.93 & 418.97 & MM \\
\hline $12-22-93$ & 1220 & E-tape & 15.1 & 0.02 & 11.25 & 418.65 & MM \\
\hline $01-20-94$ & 1640 & E-tape & 15.47 & 0.02 & 11.62 & 418.28 & MM \\
\hline $02-24-94$ & 1353 & Steel tape & 16.05 & 0.01 & 12.20 & 417.70 & MM \\
\hline $03-16-94$ & 1445 & Steel tape & 16.14 & 0.01 & 12.29 & 417.61 & MM \\
\hline $04-21-94$ & 1350 & Steel tape & 15.93 & 0.01 & 12.08 & 417.82 & MM \\
\hline $06-15-94$ & 1009 & Steel tape & 15.76 & 0.01 & 11.91 & 417.99 & MM \\
\hline
\end{tabular}


TH8 GROUND-WATER OBSERVATION WELL. (ContimUEd)

Site 10:

645112147490201 Local Number:

FC00100106DBDD1

\begin{tabular}{cccccccc}
\hline Dale & Time & Method & MP to WS & Error & LS to WS & WS elevation & Remarks \\
\hline $07-15-94$ & 0846 & Steel tape & 13.81 & 0.01 & 9.96 & 419.94 & MM \\
$08-15-94$ & 1118 & Steel tape & 13.86 & 0.01 & 10.01 & 419.89 & MM \\
$09-15-94$ & 1226 & Steel tape & 14.35 & 0.01 & 10.50 & 419.40 & MM \\
$10-18-94$ & 1445 & Steel tape & 15.53 & 0.01 & 11.68 & 418.22 & MM \\
\hline
\end{tabular}




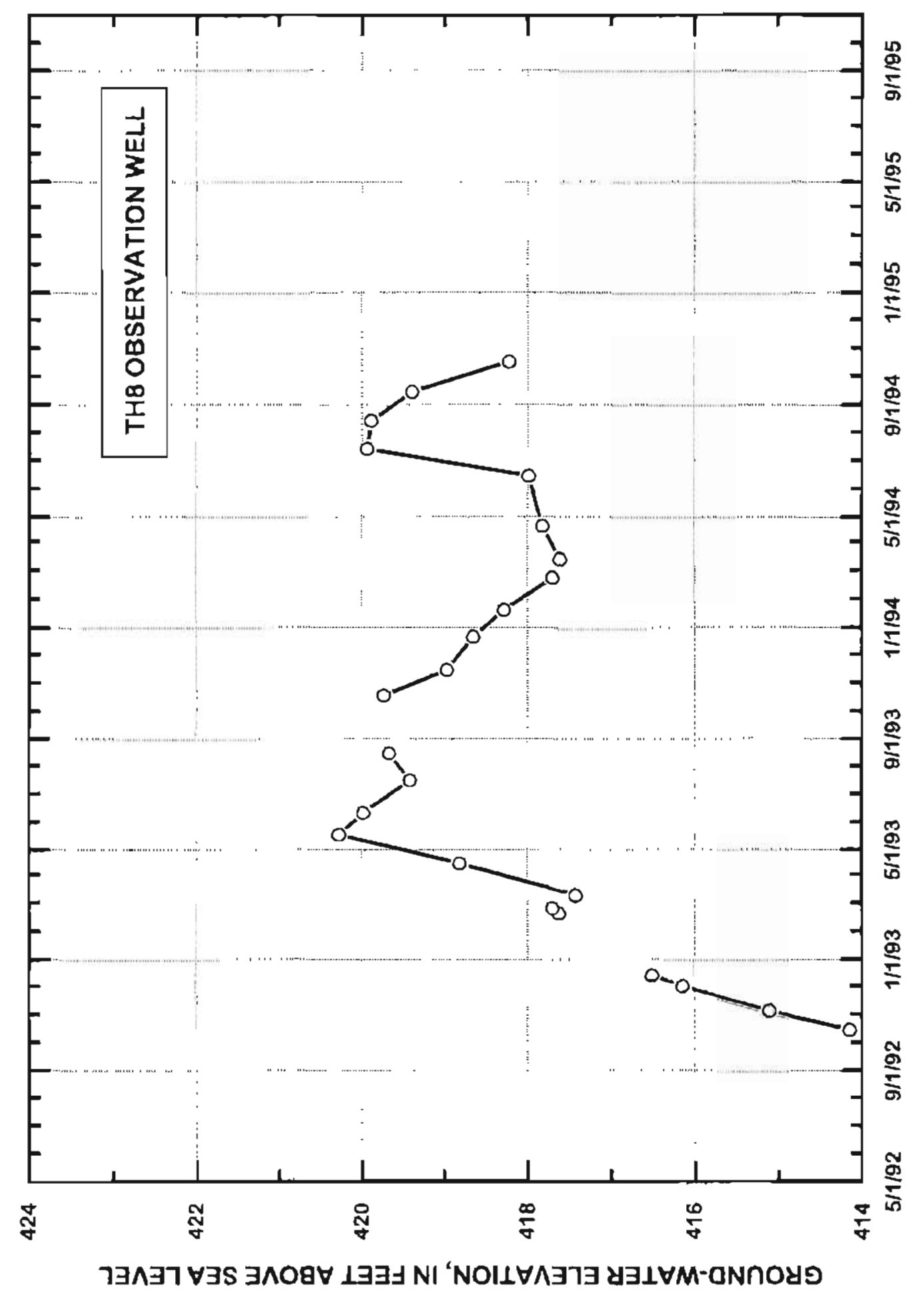


Site ID: Local Number:

\begin{tabular}{c|c} 
Feet & Elevation \\
\hline 21.2 & 413.0 \\
18.5 & 415.7 \\
20.8 & 413.5 \\
& 430.5
\end{tabular}

All measurements in feet Depth to bottom of well from MP : Depth from TOC to top of SI :

Depth from TOC to bottom of SI:

Land Surface Datum :

Datum cortections, reference survey notes in site folders

$$
\begin{array}{c|c}
\text { Date } & \text { MP Elevation (feet above sea levei) } \\
\hline 07-08-93 & 434.21
\end{array}
$$

FC00100106DACC1

LS, land surface $M M$, mass measurement MP, measuring point $N A$, not available $\mathrm{PM}$, partial moasurement $\mathrm{Sl}$, screened interval TOC, top of casing WS, water surface

\begin{tabular}{|c|c|c|c|c|c|c|c|}
\hline Date & Tme & Alethod & $\overline{M P}$ to $\mathrm{WS}$ & Error & LS to WS & WS elevation & Remarks \\
\hline $11-06-92$ & 1550 & Steel tape & 18.68 & 0.01 & 14.97 & 415.53 & $M M$ \\
\hline $12-03-92$ & 1100 & Steel tape & 17.70 & 0.01 & 13.99 & 416.51 & $M M$ \\
\hline $12-15-92$ & 1122 & Steel tape & 17.33 & 0.01 & 13.62 & 416.88 & MM \\
\hline $02-20-93$ & 1755 & Steel tape & 16.33 & 0.01 & 12.62 & 417.88 & MM \\
\hline $02-26-93$ & 1630 & Steel tape & 16.32 & 0.01 & 12.61 & 417.89 & PM \\
\hline $03-12-93$ & 1042 & Steel tape & 16.59 & 0,01 & 12.88 & 417.62 & MM \\
\hline $04-16-93$ & 1432 & Steel tape & 15.64 & 0.01 & 11.93 & 418.57 & MM \\
\hline $05-18-93$ & 1318 & Steel tape & 13.80 & 0.01 & 10.09 & 420.41 & $M M$ \\
\hline $06-11-93$ & 1135 & Steel tape & 14.14 & 0.01 & 10.43 & 420.07 & MM \\
\hline $07-17-93$ & 1420 & Steel tape & 14.63 & 0.01 & 10.92 & 419.58 & MM \\
\hline $08-16-93$ & 2005 & Steel tape & 14.35 & 0.01 & 10.64 & 419.86 & $M M$ \\
\hline $09-15-93$ & 1705 & Steal tape & 14.22 & 0.01 & 10.51 & 419.99 & MM \\
\hline $10-19-93$ & 1511 & Steel tape & 14.34 & 0.01 & 10.63 & 419.87 & MM \\
\hline $11-16-93$ & 1629 & Steel tape & 15.11 & 0.01 & 11.40 & 419.10 & MM \\
\hline $12-22-93$ & 1210 & E-tape & 15.33 & 0.02 & 11.62 & 418.88 & MAM \\
\hline $01-20-94$ & 1435 & E-tape & 15.78 & 0.02 & 12.07 & 418.43 & $M M$ \\
\hline $02-24-94$ & 1401 & Steel tape & 16.31 & 0.01 & 12.60 & 417.90 & MN \\
\hline $03-16-94$ & 1449 & Steel tape & 16.43 & 0.01 & 12.72 & $4 \$ 7.78$ & MM \\
\hline $04-21-94$ & 1347 & Steel tape & 16.43 & 0.01 & 12.72 & 417.78 & MM \\
\hline $06-15-94$ & 1003 & Steel tape & 16.08 & 0.01 & 12.37 & 418.13 & MM \\
\hline
\end{tabular}


TH9 GROUND-WATER OBSERVATION WELL (Continued)

Site ID: Local Number:

645111147485201

FCo0100106DACC1

\begin{tabular}{cccccccc}
\hline Date & Time & Method & MP to WS & Error & LS to WS & WS elevation & Remarks \\
\hline $07-15-94$ & 0841 & Steel tape & $\{4.02$ & 0.01 & 10.31 & 420.19 & MM \\
$08-15-94$ & 1122 & Steel tape & 14.12 & 0.01 & 10.41 & 420.09 & MM \\
$09-15-94$ & 1232 & Steel tape & 14.78 & 0.01 & 11.07 & 419.43 & MM \\
$10-18-94$ & 1438 & Steel tape & 15.91 & 0.01 & 12.20 & 418.30 & MM \\
\hline
\end{tabular}




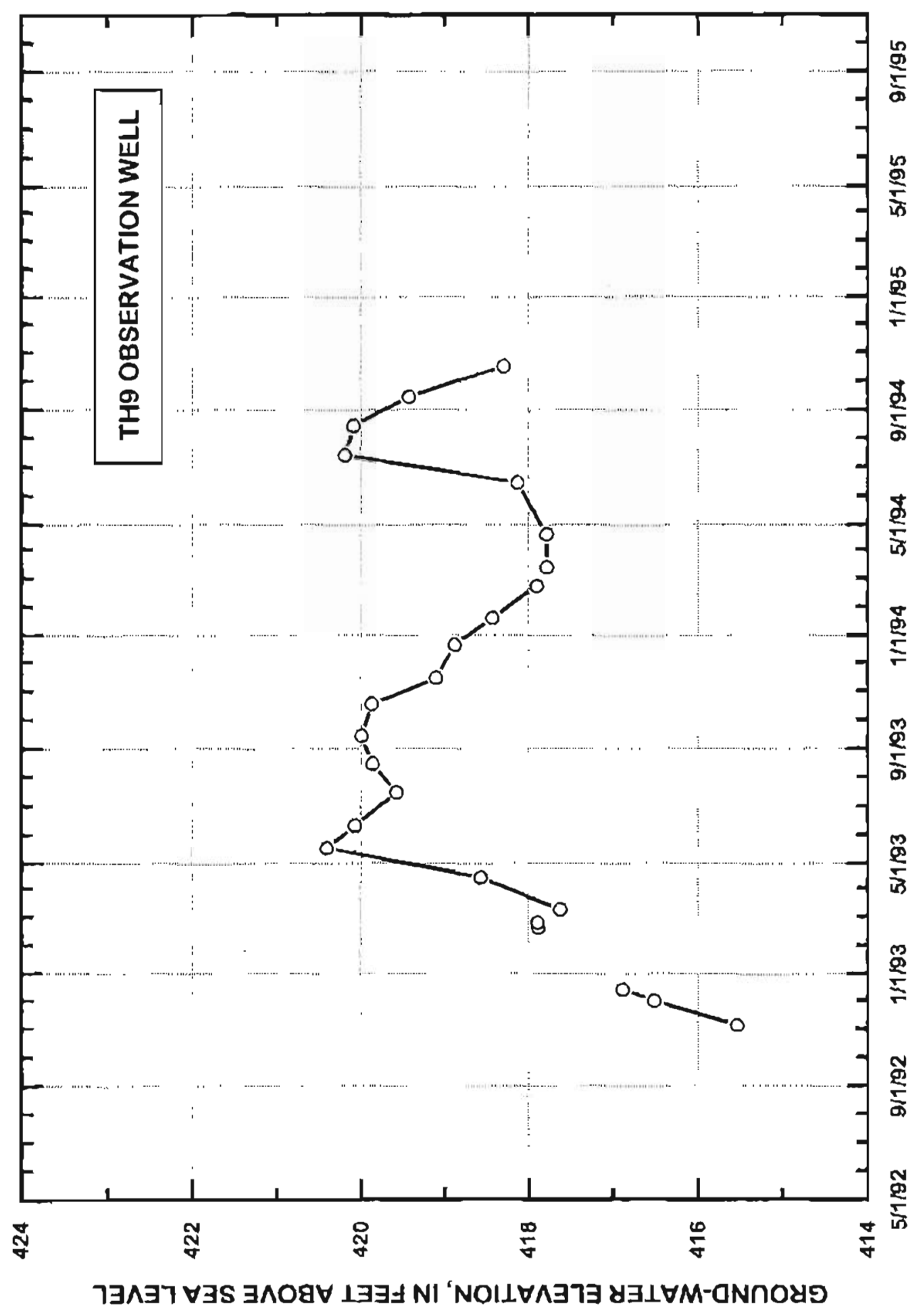


Site ID: Local Number 645115147484901

All measurements in feet

Depth to bottom of well from MP :

Depth from TOC to top of Si :

Depth from TOC to bottom of SI :

Land Surface Datum:

\begin{tabular}{c|c} 
Feet & Elevation \\
\hline 21.6 & 412.7 \\
18.9 & 415.4 \\
21.1 & 413.2 \\
& 431.8
\end{tabular}

Datum corrections, reference survey notes in site folders

$$
\begin{array}{c|c}
\text { Date } & \text { MP Elevation (feet above sea level) } \\
\hline 07-\overline{0}-93 & 434.34
\end{array}
$$

FC00100106DDBA 1

LS, land surface

$M M$, mass measurement

MP, measuring point

$N A_{4}$ not available

$P M$, partial measurement

Si, screened interval

TOC, top of casing

WS, water surface

\begin{tabular}{|c|c|c|c|c|c|c|c|}
\hline Dale & Time & Method & MP to WS & Errot & LS to WS & WS elevation & Remarks \\
\hline $11-06-92$ & 1535 & Steel tape & 18.60 & 0.01 & 16.06 & 415.74 & $\mathbf{M M}$ \\
\hline $12-03-92$ & $1\{2\}$ & Steel tape & 17.64 & 0.01 & 15.10 & 416.70 & $M M$ \\
\hline $12-14-92$ & 1058 & Steel tape & 17.33 & 0.01 & 14.79 & 417.01 & MN \\
\hline $02-20-93$ & 1100 & Steel tape & 16.41 & 0.01 & 13.87 & 417.93 & $\mathrm{MM}$ \\
\hline $02-25-93$ & 1646 & Steel tape & 16.38 & 0.01 & 13.84 & 417.96 & MM \\
\hline $03-12-93$ & 1037 & Steel tape & 18.69 & 0.01 & 14.45 & 417.65 & $M M$ \\
\hline $04-16-93$ & 1520 & Steel tape & 16.02 & 0.01 & 13.48 & 418.32 & $M M$ \\
\hline $05-18-93$ & 1328 & Steel tape & 13,88 & 0.01 & 11.34 & 420.46 & MM \\
\hline $06-11-93$ & 1218 & Steel tape & 14.32 & 0.01 & 11.78 & 420.02 & MA \\
\hline $06-30-93$ & 1855 & E-tape & 14.46 & 0.02 & 11.92 & 419.88 & PM \\
\hline $07-16-93$ & 1317 & Steel tape & 14.73 & 0.01 & 12.19 & 419.61 & $M M$ \\
\hline $08-16-93$ & 1955 & Steel tape & 14.44 & 0.01 & 11.90 & 419.90 & MM \\
\hline $09-15-93$ & 1659 & Steel tape & 14.39 & 0.01 & 11.85 & 419.95 & $\mathrm{MM}$ \\
\hline $10-19-93$ & 1427 & Steel tape & 14.47 & 0.01 & 11.93 & 419.87 & $M M$ \\
\hline $11-16-93$ & 1620 & Steel tape & 15.26 & 0.01 & 12.72 & 419.08 & MA \\
\hline $12-22-93$ & 1201 & Etape & 15.38 & 0.02 & 12.84 & 418.96 & $M M$ \\
\hline $01-20-94$ & 1445 & E-tape & 15.91 & 0.02 & 13.37 & 418.43 & MM \\
\hline $02-24-94$ & 1410 & Steel tape & 16.40 & 0.01 & 13.86 & 417.94 & $M M$ \\
\hline $03-16-94$ & 1455 & Steel tape & 16.52 & 0.01 & 13.98 & $\$ 17.82$ & MM \\
\hline $04-21-94$ & 1332 & Steel tape & 16.65 & 0.01 & 14.11 & 417.69 & MM \\
\hline
\end{tabular}


TH to GROUND-WATER OBSERVATION WELL (Continued)

Site ID:

$645115+47484901$ Locał Number:

FC00100106DDBA1

\begin{tabular}{cccccccc}
\hline Date & Time & Method & MP to WS & Error & LS to WS & WS elevation & Remarks \\
\hline $06-15-94$ & 0955 & Steel tape & 16.15 & 0.01 & 13.61 & 418.19 & MM \\
$07-15-94$ & 0836 & Steel tape & 14.07 & 0.01 & 11.53 & 420.27 & MM \\
$08-15-94$ & 1127 & Steel tape & 14.14 & 0.01 & 11.60 & 420.20 & MM \\
$09-15-94$ & 1442 & Steel tape & 14.92 & 0.01 & 12.38 & 419.42 & MM \\
$10-18-94$ & 1456 & Steel tape & 16.03 & 0.01 & 13.49 & 418.31 & MM \\
$01-18-95$ & 1455 & Steel tape & 16.73 & 0.01 & 14.19 & 417.61 & MM \\
$02-16-95$ & 1512 & Steel tape & 17.00 & 0.01 & 14.46 & 417.34 & MM \\
$05-16-95$ & 1317 & Steel tape & 15.37 & 0.01 & 12.83 & 418.97 & MM \\
$07-18-95$ & 1120 & Steel tape & 14.21 & 0.01 & 11.67 & 420.13 & MM \\
$08-15-95$ & 1353 & E-tape & 14.64 & 0.02 & 12.10 & 419.70 & MM \\
$09-14-95$ & 1235 & Steel tape & 13.37 & 0.01 & 10.83 & 420.97 & MM \\
$10-17-95$ & 1240 & Steel tape & 14.46 & 0.01 & 11.92 & 419.88 &
\end{tabular}




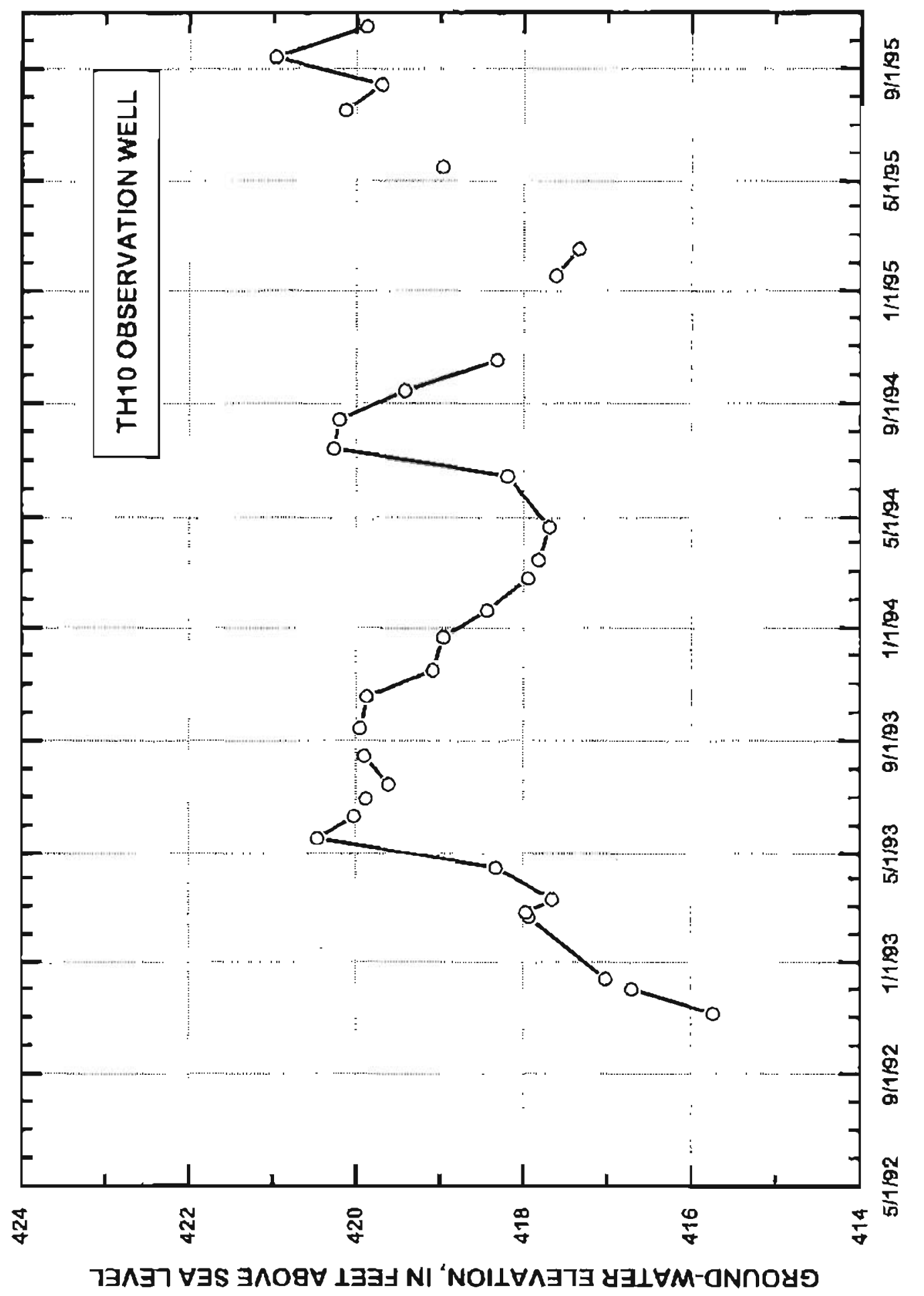


All measurements in feet Depth to bottom of well from MP : Depth fon TOC to top of SI: Depth from TOC to bottom of SI: Land Surface Datum :

\begin{tabular}{c|c} 
Feet & Elevation \\
\hline NA & NA \\
NA & NA \\
NA & NA \\
& 429.1
\end{tabular}

Datum corrections, reference survey notes in site folders

$$
\begin{array}{c|c}
\text { Dale } & \text { MP Elevation (feet above sea levei) } \\
\hline 12-12-95 & 432.34
\end{array}
$$

LS, land surface $M M$, mass measurement $M P$, measuring point $N A$ not available PM, partial measurement SI, screened intervał TOC, top of casing WS, water surface

\begin{tabular}{cccccccc}
\hline Date & Time & Method & MP to WS & Error & LS to WS & WS elevation & Remarks \\
\hline $09-15-94$ & 1137 & Steel tape & 14.23 & 0.01 & 10.99 & 418.11 & MM \\
$10-18-94$ & 1641 & Steel tape & 15.00 & 0.01 & 12.76 & 416.34 & MM \\
$11-16-94$ & 1435 & Steel tape & 11.62 & 0.01 & 8.38 & 420.72 & MM \\
$12-23-94$ & 1443 & Steel tape & 15.56 & 0.01 & 12.32 & 416.78 & MM \\
$01-18-95$ & 1418 & Steel tape & 16.10 & 0.01 & 12.86 & 416.24 & MM \\
$02-16-95$ & 1528 & Steel tape & 16.29 & 0.01 & 13.05 & 416.05 & MM \\
$04-15-95$ & 1235 & Steel tape & 16.24 & 0.01 & 13.00 & 416.10 & MM \\
$05-16-95$ & 1400 & Steel tape & 14.09 & 0.01 & 10.85 & 418.25 & MM \\
$08-15-95$ & 1558 & E-tape & 13.33 & 0.02 & 10.09 & 419.01 & MM \\
$09-14-95$ & 1213 & Steel tape & 12.06 & 0.01 & 8.82 & 420.28 & MM \\
$10-17-95$ & 1033 & Steet tape & 14.56 & 0.01 & 11.32 & 417.78 & MM \\
$11-21-95$ & 1039 & Steel tape & 14.77 & 0.01 & 11.53 & 417.57 \\
$02-14-96$ & 1445 & Steel tape & 15.66 & 0.01 & 12.42 & 416.68 & MM \\
$03-19-96$ & 1400 & E-tape & 15.47 & 0.01 & 12.23 & 416.87 \\
$04-22-96$ & 1157 & E-tape & 15.40 & 0.01 & 12.16 & 416.94 & MM \\
\hline
\end{tabular}




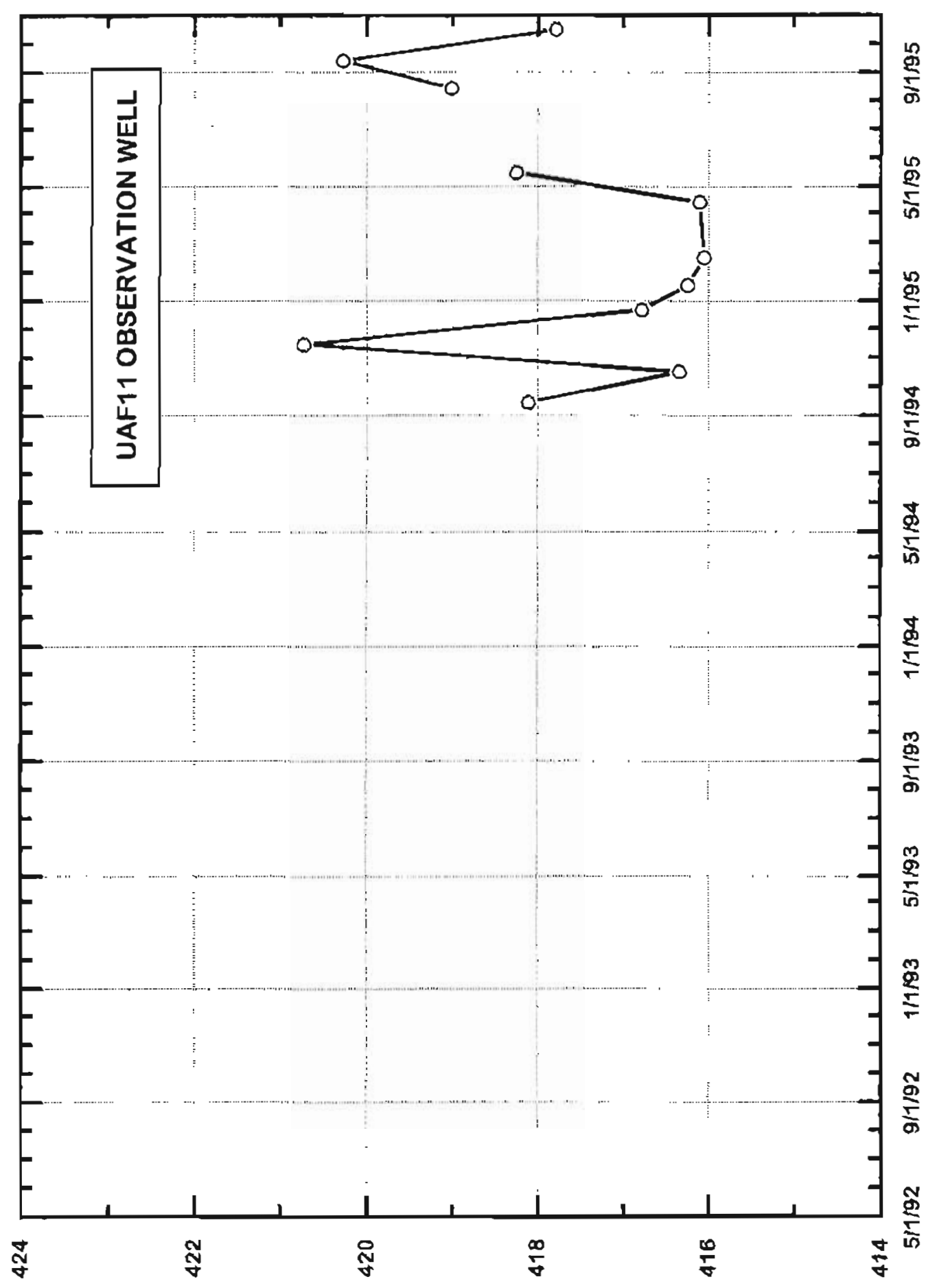

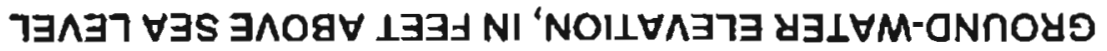


CU1 SLOUGH OBSERVATION SITE

Station ID:

1551401760

CULVERT SITE

All measurements in feat

Culvert diameter.

5.50

Land Surface Datum:

419.18

LS, fand surface

$M M$, mass measurement

MP, measuring polnt

Datum corrections, reference survey notes in site folders

NA, not available

$\mathrm{PM}$, partial measurement

\begin{tabular}{c|c} 
Date & MP Elevation (feet above sea level) \\
\hline $07.01-92$ & 424.68
\end{tabular}

WS, water surface

\begin{tabular}{|c|c|c|c|c|c|c|c|}
\hline Date & Time & Method & MP to WS & Error & LS to WS & WS elevation & Remarks \\
\hline $06-01-92$ & 2057 & Culvert & -1.55 & 0.01 & 3.95 & $423 . \overline{13}$ & PM \\
\hline $06-02-92$ & 0720 & Culvert & -1.58 & 0.01 & 3.92 & 423.10 & PM \\
\hline $06-02-92$ & 2300 & Culvert & -1.50 & 0.01 & 4.00 & 423.18 & PM \\
\hline $06-04-92$ & 0800 & Culvert & -0.96 & 0.01 & 4.54 & 423.72 & PM \\
\hline $06-16-92$ & 0800 & Culvert & $N A$ & 0.01 & NA & NA & PM, filled with debris \\
\hline $06-17-92$ & 0910 & Culvert & NA & 0.01 & NA & NA & PM, fitled with debris \\
\hline $06-22-92$ & 0855 & Cutvert & NA & 0.01 & NA & NA & PM, filed with debris \\
\hline $07-17-92$ & 1203 & Cutvert & -5.07 & 0.01 & 0.43 & 419.61 & MM \\
\hline $08-13-92$ & 1925 & Cutvert & -4.89 & 0.01 & 0.61 & 419.79 & MM \\
\hline $11-05-92$ & 1120 & Cutvert & NA & 0.01 & NA & $\mathrm{NA}$ & PM, frozen, muddy bottom \\
\hline $06-03-93$ & 1115 & Culvert & NA & 0.01 & NA & NA & PM, dry near culvert \\
\hline $06-03-93$ & 1510 & Culvert & -4.91 & 0.01 & 0.59 & 419.77 & PM, used eye level \\
\hline $07-16-93$ & 1136 & Culvert & NA & 0.01 & NA & NA & $\mathrm{MN}_{2} \mathrm{~d} y \mathrm{y}$ \\
\hline $07-28-93$ & 1756 & Culvert & NA & 0.01 & NA & NA & $\mathrm{PM}_{\varepsilon}$ filled with debris \\
\hline $08-16-93$ & 1120 & Cuivert & -4.55 & 0.01 & 0.95 & 420.13 & $M M$ \\
\hline $10-19-93$ & 1352 & Culvert & NA & 0.01 & NA & NA & $M M, d r y$ \\
\hline $01-20-94$ & 1348 & Cutvert & NA & 0.01 & NA & NA & $M M_{1}$ frozen \\
\hline $04-21-94$ & 1415 & Culvert & -4.47 & 0.01 & 1.03 & 420.21 & MM \\
\hline $06-09-94$ & $\uparrow 725$ & Culver & NA & 0.01 & NA & $N A$ & PM \\
\hline $06-15-94$ & 0845 & Culvert & NA & 0.01 & NA & NA & MM \\
\hline $06-21-94$ & $163 \uparrow$ & Culvert & -2.84 & 0.01 & 2.66 & 421.84 & $P M$ \\
\hline $06-27-94$ & 1559 & Culvert & -2.08 & 0.01 & 3.42 & 422.60 & PM \\
\hline $07-01-94$ & 4505 & Culvert & -3.35 & 0.01 & 2.15 & 421.33 & PM \\
\hline
\end{tabular}


CU' SLOUGH OBSERVATION SITE

Station ID:

1551401760 CULVERT SITE

\begin{tabular}{|c|c|c|c|c|c|c|c|}
\hline Date & Time & Method & MP to WS & Error & LS to WS & WS elevation & Remarks \\
\hline $0 \overline{7-15-94}$ & 1103 & Cuvert & -4.90 & 0.01 & 0.60 & 419.78 & MA \\
\hline $08-05-94$ & 1600 & Cutvert & -4.45 & 0.01 & 1.05 & 420.23 & PM \\
\hline $08-12-94$ & 1610 & Cutvert & -3.91 & 0.01 & 1.59 & 420.77 & PM \\
\hline $08-15-94$ & 1026 & Culvert & -4.52 & 0.01 & 0.98 & 420.16 & MW \\
\hline $09-15-94$ & 1130 & Cutvert & NA & 0.01 & NA & NA & MM \\
\hline $10-18-94$ & 1629 & Culvert & NA & 0.01 & NA & $\mathrm{HA}$ & $\mathrm{MM}, \mathrm{dry}$ \\
\hline $11-16-94$ & 1411 & Culvert & NA & 0.01 & NA & $\mathrm{NA}$ & $M M, d r y$ \\
\hline $12-23-94$ & 1430 & Cutvert & NA & 0.01 & NA & NA & MA, dry \\
\hline $01-18-95$ & 1410 & Culvert & NA & 0.01 & NA & NA & MN; dy \\
\hline $02-16-95$ & 1538 & Culvert & NA & 0.01 & NA & NA & MM, dry \\
\hline $04-15-95$ & 1240 & Culvert & NA & 0.01 & NA & NA & MM, snow on culvert \\
\hline $05-16-95$ & 1410 & Culvert & NA & 0.01 & $\mathrm{NA}$ & NA & MM, dry \\
\hline $07-17-95$ & 1426 & Culvert & -3.87 & 0.01 & 1.63 & 420.81 & MM \\
\hline $08-15-95$ & 1608 & Culvert & -5.46 & 0.01 & 0.04 & 419.22 & $\mathrm{MM}$ \\
\hline $09-14-95$ & 1227 & Culvert & -4.69 & 0.01 & 0.81 & 419.99 & MM \\
\hline $10-17-95$ & 1042 & Culvert & NA & 0.01 & $\mathrm{NA}$ & $N A$ & $\mathrm{MM}_{\mathrm{r}} \mathrm{dry}$ \\
\hline
\end{tabular}


WATER ELEVATION, IN FEET ABOVE SEA LEVEL

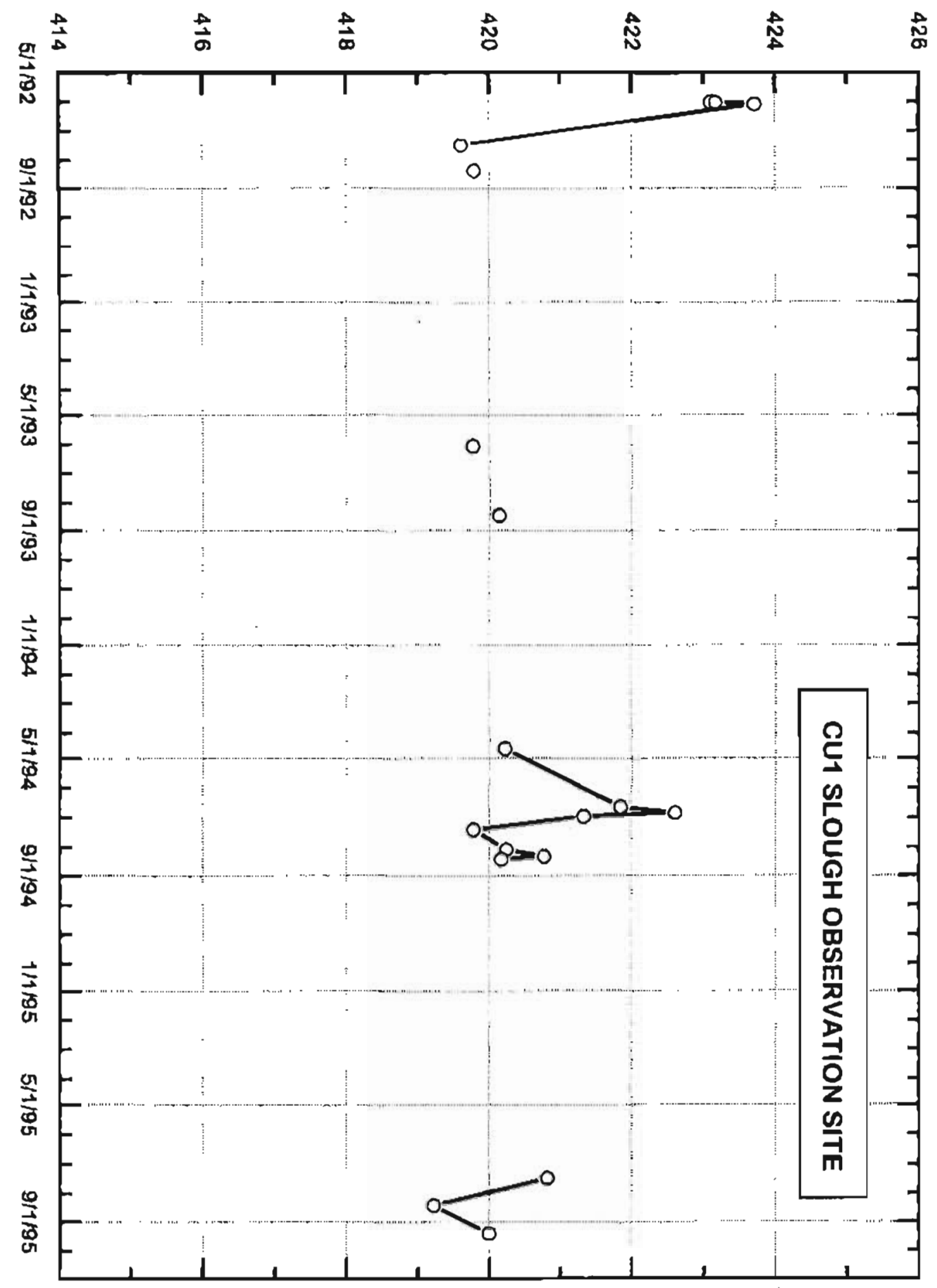




\section{CULVERT SITE}

Ali measurements in feet

Culvert diameter:

5.50

Land Surface Datum:

419.18

Datum corrections, reference survey notes in site folders

Date

MP Elevation (feet above sea level)
$S$, and surface MM, mass measurement

MP. measuring point

$N A$, not avaitable

PM, partial measurement

WS, water surface

\begin{tabular}{|c|c|c|c|c|c|c|c|}
\hline Date & Tinne & Method & MP to WS & Error & LS to WS & WS elevation & Remarks \\
\hline $06-01-92$ & 2057 & Culvert & -1.40 & $\overline{0.01}$ & 4.10 & $423 . \uparrow 1$ & $\mathrm{PM}$ \\
\hline $06-02-92$ & 0720 & Culvert & -1.44 & 0.01 & 4,06 & 423.07 & PM \\
\hline $06-02-92$ & 2300 & Culvert & -1.36 & 0.01 & 4.14 & 423.15 & PM \\
\hline $06-04-92$ & 0800 & Cukert & -0.80 & 0.01 & 4.70 & 423.71 & $\mathrm{PM}$ \\
\hline $06-16-92$ & 0800 & Cukvert & -5.28 & 0.01 & 0.22 & 419.23 & $P M$ \\
\hline $06-17-92$ & 0910 & Culvent & NA & 0.01 & NA & NA & MM, perched \\
\hline $06-22-92$ & 0855 & Cutvert & -5.09 & 0.01 & 0.41 & 419.42 & $P M$ \\
\hline $07-17-92$ & 1206 & Culvert & -4.14 & 0.01 & 1.39 & 420.40 & $\mathbf{P M}$ \\
\hline $08-13-92$ & 1934 & Culvert & -4.98 & 0.01 & 0.52 & 419.53 & MM \\
\hline $11-05-92$ & 1120 & Culvert & NA & 0.01 & NA & $\mathrm{NA}$ & PM, frozen \\
\hline $06-03-93$ & 1115 & Culvert & NA & 0.01 & NA & NA & $P M, d r y$ \\
\hline $07-16-93$ & 1136 & Culvert & NA & 0.01 & NA & NA & MW dry \\
\hline $07-28-93$ & 1756 & Culvert & -4.85 & 0.01 & 0.65 & 419.66 & PM \\
\hline $08-16-93$ & 1120 & Culvert & -4.47 & 0.01 & 1.03 & 420.04 & MM, stagnant \\
\hline $10-19-93$ & 1352 & Culvert & NA & 0.01 & NA & NA & MM, dry \\
\hline $01-20-94$ & 1348 & Culvert & NA & 0.01 & NA & NA & MM, frozen \\
\hline $04-21-94$ & 1415 & Culvert & -4.60 & 0.01 & 0.80 & 419.91 & MM \\
\hline $06-09-94$ & 1725 & Cuivert & $N A$ & 0.01 & NA & NA & PM \\
\hline $06-15-94$ & 0845 & Culvert & NA & 0.01 & NA & NA & $M M$ \\
\hline $06-21-94$ & 1631 & Culvert & -2.57 & 0.04 & 2.83 & 421.94 & $P M$ \\
\hline $06-27-94$ & 1502 & Culvert & -1.90 & 0.01 & 3.60 & 422.61 & $\mathrm{PM}$ \\
\hline $07-01-94$ & 1505 & Culvert & -3.19 & 0.01 & 2.31 & 421.32 & PM \\
\hline $07-15-94$ & 1103 & Culvert & -5.41 & 0.01 & 0.09 & 419.10 & MM \\
\hline
\end{tabular}


CU2 SLOUGH OBSERVATION SITE

(Continued)

Station ID:

1551401760

\begin{tabular}{|c|c|c|c|c|c|c|c|}
\hline Date & Time & Method & MP to WS & Eтог & LS to WS & WS elevation & Remarks \\
\hline $08-05-94$ & 1600 & Culver & -4.14 & 0.01 & 1.36 & 420.37 & PM \\
\hline $08-12-94$ & 1810 & Culvert & -3.74 & 0.01 & 1.76 & 420.77 & PM \\
\hline $08-15-94$ & t026 & Cutvert & -4.38 & 0.01 & 1.12 & 420.13 & MM \\
\hline $09-15-94$ & 1130 & Culvert & NA & 0.01 & $\mathrm{NA}$ & $N A$ & $\mathrm{MM}$ \\
\hline $10-18-94$ & 1629 & Culvert & $\mathrm{NA}$ & 0.01 & $\mathrm{NA}$ & NA & $M M_{1} d r y$ \\
\hline $11-16-94$ & 1411 & Culvert & NA & 0.01 & $\mathrm{NA}$ & NA & MM, dry \\
\hline $12-23-94$ & 1430 & Culvert & NA & 0.01 & $\mathrm{NA}$ & NA & $M M, d r y$ \\
\hline $01-18-95$ & 1410 & Culvert & NA & 0.01 & NA & NA & MAM, dry \\
\hline $02-16-95$ & 1538 & Culvert & $N A$ & 0.01 & NA & NA & $M M_{1}$ dry \\
\hline $04-15-95$ & 1240 & Cukert & -4.21 & 0.01 & 1.29 & 420.30 & $M M$ \\
\hline $05-16-95$ & 1410 & Culvert & $\mathrm{NA}$ & 0.01 & $\mathrm{NA}$ & NA & ABA, dry \\
\hline $07-17-95$ & 1430 & Culvert & -3.73 & 0.01 & 1.77 & 420.78 & MM \\
\hline $08-15-95$ & 1608 & Culvert & NA & 0.01 & NA & $\mathrm{NA}$ & $M M, d r y$ \\
\hline $09-14-95$ & $\$ 229$ & Culvert & NA & 0.01 & NA & NA & $M M, d r y$ \\
\hline $10-17-95$ & 1042 & Culvert & NA & 0.01 & NA & NA & $M M, d r y$ \\
\hline
\end{tabular}




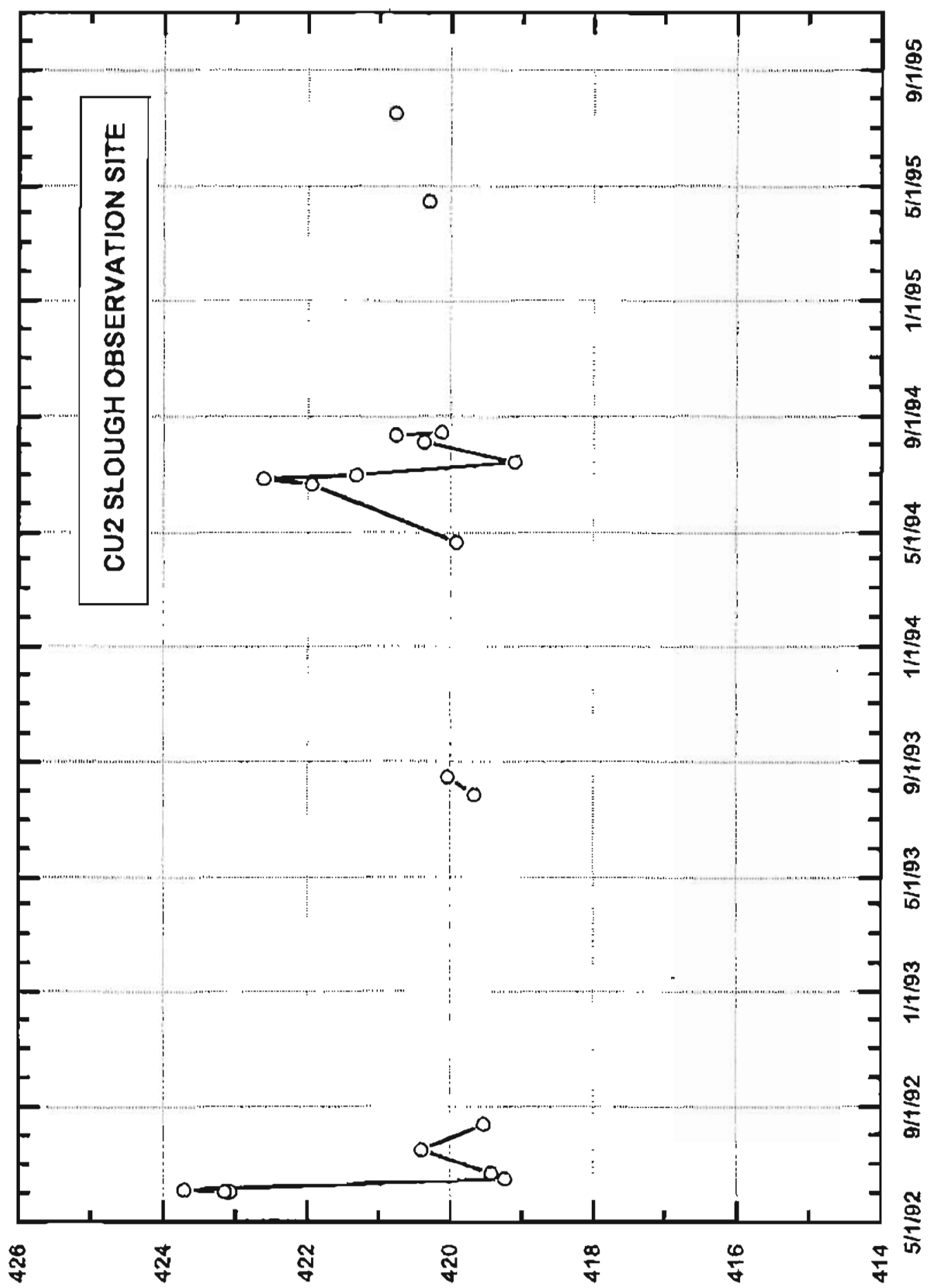

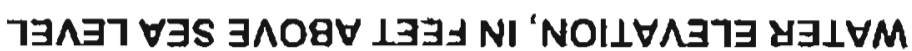


All measurements in feet

Culvert dlameter: $\quad 4.00$

Land Surace Datum:

422.36

Datum corrections, reference survey notes in site folders:

\begin{tabular}{c|c} 
Date & MP Elevation (feet above sea level) \\
\hline $07-01-92$ & 426.36
\end{tabular}
LS, land surface $M M$, mass measurement $M P$, measuring point NA, not available PM, partial measurement WS, water surface

\begin{tabular}{cccccccc}
\hline Date & Time & Method & MP to WS & Error & LS to WS & WS elevation & Remarks \\
\hline $06-01-92$ & 2057 & Culvert & -3.24 & 0.01 & 0.76 & 423.12 & PM \\
$06-02-92$ & 0720 & Culvert & -3.28 & 0.01 & 0.72 & 423.08 & PM \\
$06-02-92$ & 2300 & Culvert & -3.20 & 0.01 & 0.80 & 423.16 & PM \\
$06-04-92$ & 0800 & Culvert & -2.64 & 0.01 & 1.36 & 423.72 & PM \\
$06-16-92$ & 0800 & Culvert & NA & 0.01 & NA & NA & Dry \\
$06-17-92$ & 0910 & Culvert & -5.10 & 0.01 & -1.10 & 421.26 & PM \\
$06-22-92$ & 0855 & Culvert & NA & 0.01 & NA & NA & Perched \\
$08-13-92$ & 1937 & Culvert & NA & 0.01 & NA & NA & MM, perched \\
$11-05-92$ & 1120 & Culvert & NA & 0.01 & NA & NA & Frozen \\
$06-03-93$ & 1115 & Culvert & NA & 0.01 & NA & NA & PM, dry \\
$07-16-93$ & 1136 & Culvert & NA & 0.01 & NA & NA & MM, dry \\
$07-28-93$ & 1756 & Culvert & NA & 0.01 & NA & NA & PM, dry \\
$08-16-93$ & 1120 & Culvert & NA & 0.01 & NA & NA & MM, dry \\
$10-19-93$ & 1352 & Culvert & NA & 0.01 & NA & NA & MM, dry \\
$01-20-94$ & 1348 & Culvert & NA & 0.01 & NA & NA & MM, frozen \\
$04-21-94$ & 1415 & Culvert & -3.58 & 0.01 & 0.42 & 422.78 & MM \\
$06-09-94$ & 1725 & Culvert & NA & 0.01 & NA & NA & PM \\
$06-15-94$ & 0945 & Culvert & NA & 0.01 & NA & NA & MM \\
$06-21-94$ & 1631 & Culvert & -4.38 & 0.01 & -0.38 & 421.98 & PM \\
$06-27-94$ & 1605 & Culvert & -5.73 & 0.01 & -1.73 & 420.63 & PM \\
$07-01-94$ & 1505 & Culvert & NA & 0.01 & NA & NA & PM, dry \\
$07-15-94$ & 1103 & Cuivert & NA & 0.01 & NA & NA & NM \\
$08-05-94$ & 1600 & Cuivert & NA & 0.01 & NA & NA & PM, dry
\end{tabular}




\begin{tabular}{|c|c|c|c|c|c|c|c|}
\hline $\begin{array}{l}\text { CU3 SLOUQ } \\
\text { CULVERT }\end{array}$ & BSERV & SITE & & ontinut & & Station ID: & 1551401760 \\
\hline Date & Time & Method & MP to WS & Error & LS to WS & WS elevation & Remarks \\
\hline $08-12-94$ & 1610 & Culvert & NA & $0 . \overline{01}$ & $\mathrm{NA}$ & NA & PM, dry \\
\hline $08-15-94$ & 1214 & Cukert & NA & 0.01 & NA & NA & MM \\
\hline $09-15-94$ & 1130 & Cuivert & NA & 0.01 & NA & NA & $\mathrm{MM}$ \\
\hline $10-18-94$ & 1629 & Culvert & NA & 0.01 & NA & NA & $M M, d r y$ \\
\hline $11-16-94$ & 1411 & Culvert & NA & 0.01 & NA & NA & $M M_{1}$ dry \\
\hline $12-23-94$ & 1430 & Cukvert & NA & 0.01 & NA & NA & $M M, d r y$ \\
\hline $01-18-95$ & 1410 & Cuhert & NA & 0.01 & NA & NA & $M M, d r y$ \\
\hline $02-16-95$ & 1538 & Culvert & NA & 0.01 & NA & NA & MM, dry \\
\hline $04-15-95$ & 1240 & Culvert & -3.61 & 0.01 & 0.39 & 422.75 & $M M$ \\
\hline $05-16-95$ & 1410 & Culvert & NA & 0.01 & NA & NA & $M M, d r y$ \\
\hline $07-17-95$ & 1432 & Culvert & NA & 0.01 & NA & NA & MM, dry \\
\hline $08-15-95$ & 1608 & Culvert & $\mathrm{NA}$ & 0.01 & NA & NA & $M M, d r y$ \\
\hline $09-14-95$ & 1225 & Culvert & -4.87 & 0.01 & -0.87 & 421.49 & $M M, d r y$ \\
\hline $10-17-95$ & 1042 & Cuivert & $\mathrm{NA}$ & 0.01 & NA & NA & MM. dry \\
\hline
\end{tabular}




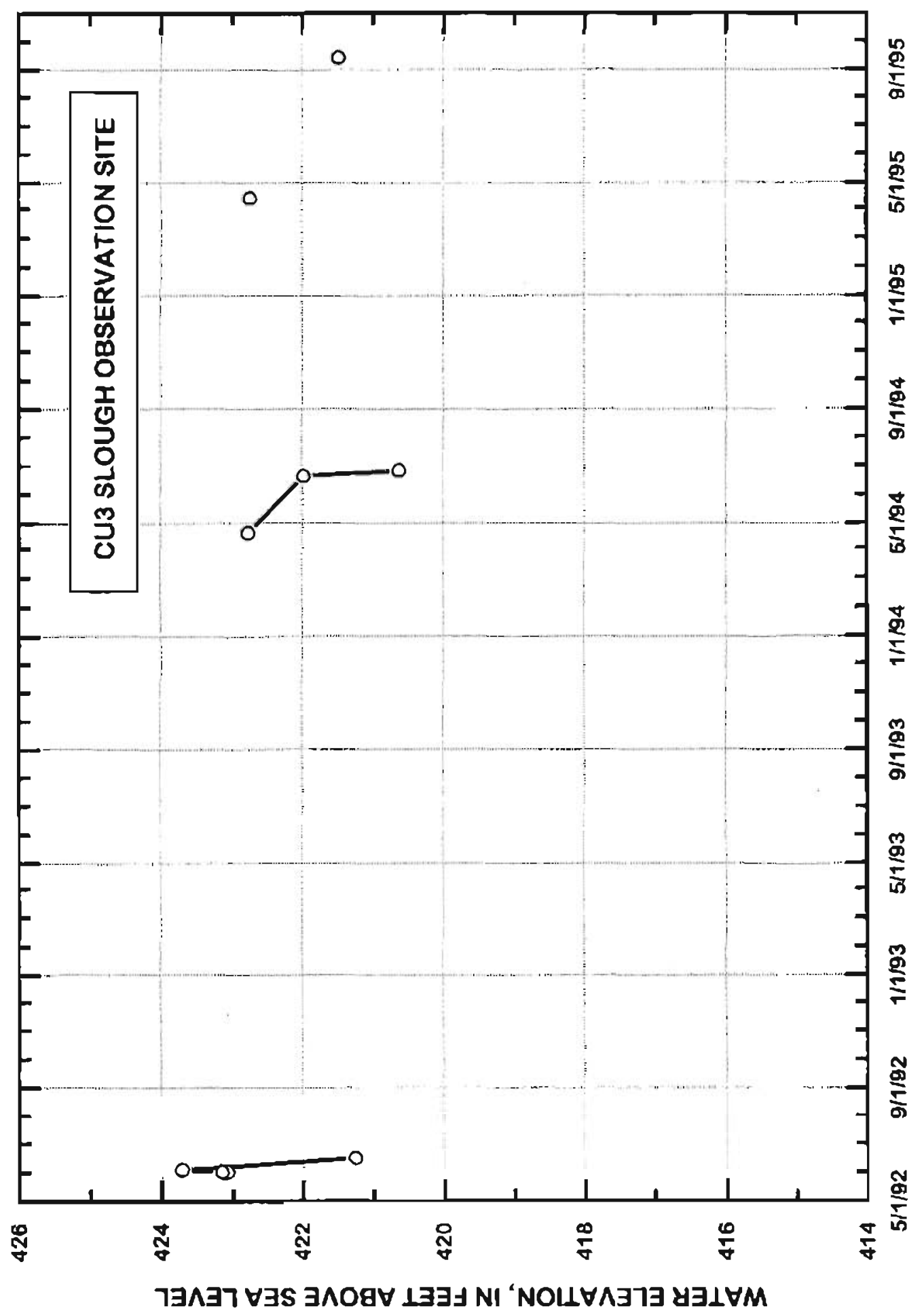

WATER-ELEVATION TABLES AND HYDROGAAPHS 186 
All measurements in feet

Culvert diameter:

5.50

Land Surface Datum:

418.74

Datum corrections, reference survey notes in site folders

\begin{tabular}{c|c} 
Date & MP Elevation (feet above sea level) \\
\hline $08-17-93$ & 424.21 \\
$06-23-94$ & 424.45
\end{tabular}

LS, land surface

MM, mass measurement

MP, measuring point

$N A$, not available

$P M$, partial measurement

WS, water surface

\begin{tabular}{|c|c|c|c|c|c|c|c|}
\hline Date & Time & Method & MP to WS & Error & LS to WS & WS elevation & Remarks \\
\hline $06-02-92$ & 0750 & Cutvert & -1.21 & 0.01 & 4.29 & $42 \overline{3.00}$ & PM \\
\hline $06-02-92$ & 2305 & Culvert & -1.13 & 0.01 & 4.37 & 423.08 & PM \\
\hline $06-04-92$ & 0942 & Culvert & -0.59 & 0.01 & 4.91 & 423.62 & $\mathrm{PM}$ \\
\hline $06-08-92$ & 1819 & Culvert & -3.29 & 0.01 & 2.21 & 420.92 & $\mathrm{PM}$ \\
\hline $06-16-92$ & 0816 & Culvert & -3.58 & 0.01 & 1.92 & 420.63 & $\mathrm{PM}$ \\
\hline $06-17-92$ & 0830 & Culvert & -3.61 & 0.01 & 1.89 & 420.60 & $\mathrm{PM}$ \\
\hline $06-22-92$ & 0810 & Culvert & -3.52 & 0.01 & 1.98 & 420.69 & PM \\
\hline $07-17-92$ & 1058 & Culvert & -5.32 & 0.01 & 0.18 & 418.89 & $\mathbf{P M}$ \\
\hline $11-05-92$ & 1135 & Culvert & NA & 0.01 & NA & NA & PM, frozen, muddy bottom \\
\hline $07-16-93$ & 1327 & Culvert & NA & 0.01 & NA & NA & MM, dry \\
\hline $08-16-93$ & 1148 & Culvert & NA & 0.01 & NA & NA & $\mathrm{MM}_{\mathrm{L}} \mathrm{dry}$ \\
\hline $10-19-93$ & 1420 & Culvert & NA & 0.01 & NA & NA & $M M$, frozen \\
\hline $11-16-93$ & 1330 & Culvert & NA & 0.01 & NA & NA & MM, frozen \\
\hline $01-20-94$ & 1330 & Culvert & NA & 0.01 & NA & NA & $M M$, frozen \\
\hline $02-24-94$ & 1437 & Culvert & NA & 0.01 & NA & NA & MM, frozen \\
\hline $03-16-94$ & 1335 & Cutvert & NA & 0.01 & NA & NA & MM, frozen \\
\hline $04-21-94$ & $\uparrow 313$ & Cutivert & -1.51 & 0.01 & 3.99 & 422.70 & MM \\
\hline $06-09-94$ & 1701 & Culvert & NA & 0.01 & $\mathrm{NA}$ & NA & PM \\
\hline $06-15-94$ & 0858 & Culvert & NA & 0.01 & $\mathrm{NA}$ & NA & MN \\
\hline $06-21-94$ & 1649 & Cuvert & $N A$ & 0.04 & NA & NA & $P M_{1} d r y$ \\
\hline
\end{tabular}


CU4 SLOUGH OBSERVATION SITE

Station ID:

1551401710

\begin{tabular}{cccccccc} 
CULVERT SITE & \multicolumn{7}{c}{} \\
\hline Date & Time & Methor & MP to WS & Error & LS to WS & WS elevation & Remarks \\
\hline $06-27-94$ & 1615 & Culvert & -1.75 & 0.01 & 3.75 & 422.70 & PM \\
$07-15-94$ & 0913 & Culvert & NA & 0.01 & NA & NA & MM, dry \\
$08-05-94$ & 1635 & Culvert & NA & 0.01 & NA & NA & MM, dry \\
$08-15-94$ & 1104 & Culvert & NA & 0.01 & NA & NA & MM, dry \\
$09-15-94$ & 1153 & Cutvert & NA & 0.01 & NA & NA & MM, dry \\
$10-18-94$ & 1505 & Culvert & NA & 0.01 & NA & NA & MM, dry \\
$11-16-94$ & 1437 & Cutvert & NA & 0.01 & NA & NA & MM, dry \\
$12-23-94$ & 1440 & Cutvert & NA & 0.01 & NA & NA & MM, dry \\
$01-18-95$ & 1444 & Culvert & NA & 0.01 & NA & NA & MM, dry \\
$02-16-95$ & 1539 & Culvert & NA & 0.01 & NA & NA & MM, dry \\
$04-15-95$ & 1303 & Culvert & -2.97 & 0.01 & 2.53 & 421.48 & MM \\
$05-16-95$ & 1319 & Culvert & NA & 0.01 & NA & NA & MM, dry \\
$06-14-95$ & 1455 & Culvert & NA & 0.01 & NA & NA & MM, dry \\
$07-18-95$ & 1101 & Culvert & -4.05 & 0.01 & 1.45 & 420.40 & MM \\
$08-15-95$ & 1437 & Culvert & NA & 0.01 & NA & NA & MM, dry \\
$09-14-95$ & 1201 & Culvert & -3.35 & 0.01 & NA & 421.10 & MM \\
$10-17-95$ & 1050 & Culvert & NA & 0.01 & NA & NA & MM, dry \\
\hline
\end{tabular}




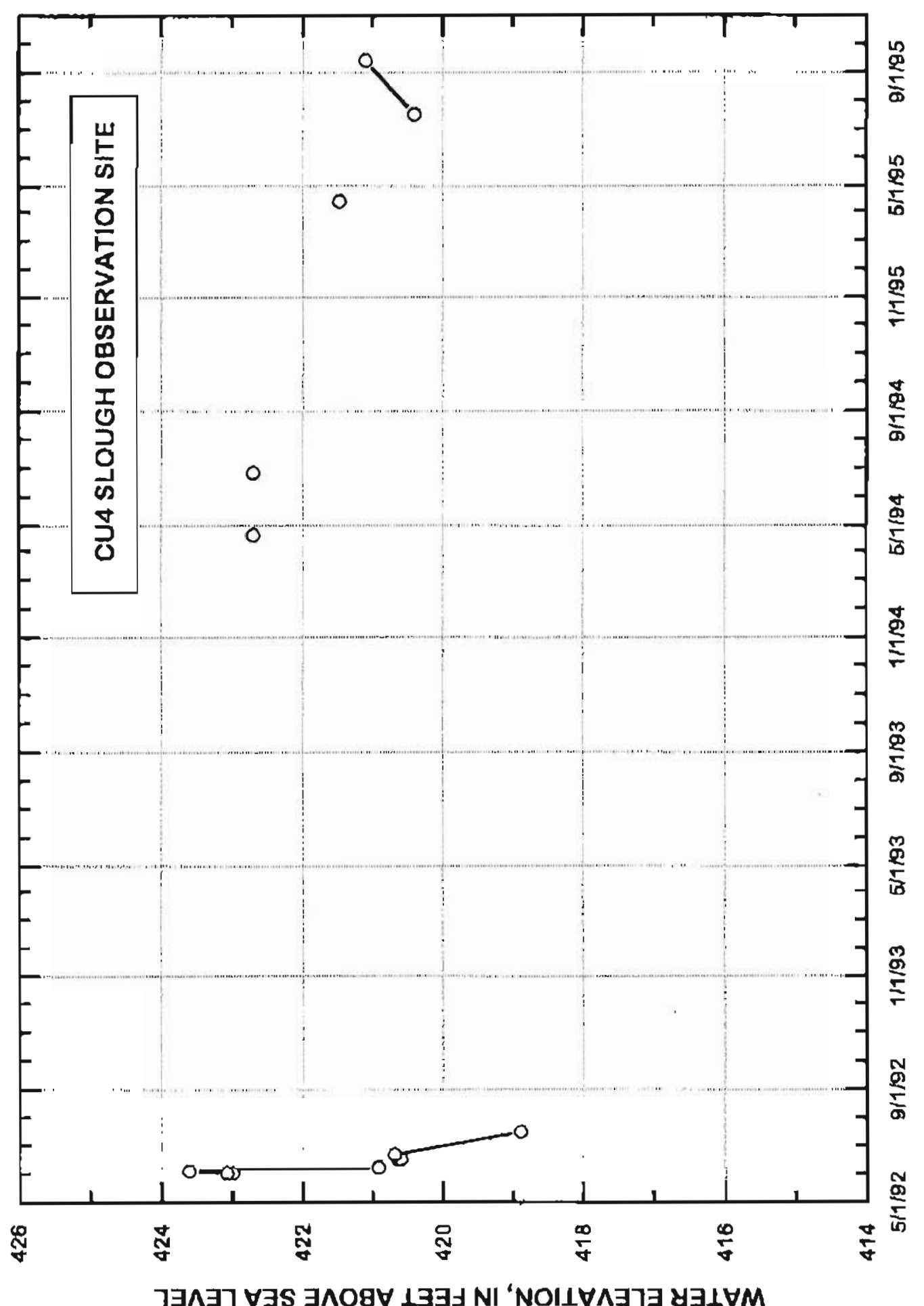


CU5 SLOUGH OBSERVATION SITE

Station ID:

1551401730

CULVERT SITE

All measurements in feet

Culvert diameter: $\quad 3.00$

Land Surface Datum : $\quad \mathbf{4 1 9 . 1 8}$

LS, land surface

$M M$, mass measurement

MP, measuring polnt

Datum corrections, reference survey notes in site folders

NA, not avallable

$P M$, partial measurement

WS, water surface

\begin{tabular}{c|c} 
Date & MP Elevation (feet above sea level) \\
\hline $07-01-92$ & 422.18
\end{tabular}

\begin{tabular}{cccccccc}
\hline Date & Time & Method & MP to WS & Error & LS to WS & WS elevation & Remarks \\
\hline $06-17-92$ & 0845 & Culvert & -1.65 & 0.01 & 1.35 & 420.53 & PM \\
$06-22-92$ & 0819 & Culvert & -1.65 & 0.01 & 1.35 & 420.53 & PM \\
$07-17-92$ & 1132 & Culvert & NA & 0.01 & NA & NA & PM, damp \\
$08-13-92$ & 1911 & Culvert & -2.42 & 0.01 & 0.58 & 419.76 & PM \\
$11-05-92$ & 1030 & Cuivert & NA & 0.01 & NA & NA & PM, dry \\
$06-03-93$ & 1128 & Culvert & -2.33 & 0.01 & 0.67 & 419.85 & PM \\
$07-01-93$ & 1326 & Culvert & -1.50 & 0.01 & 1.50 & 420.68 & PM \\
$07-17-93$ & 1317 & Culvert & NA & 0.01 & NA & NA & MM, dry \\
$08-16-93$ & 1100 & Culvert & -2.30 & 0.01 & 0.70 & 419.88 & MM, stagnant water \\
$10-19-93$ & 1340 & Culvert & NA & 0.01 & NA & NA & PM, frozen \\
$01-20-94$ & 1335 & Culvert & NA & 0.01 & NA & NA & MM, frozen \\
$04-21-94$ & 1425 & Culvert & -1.83 & 0.01 & 1.17 & 420.35 & MM, slow moving water \\
$06-09-94$ & 1709 & Culvert & N/A & 0.01 & NA & NA & PM \\
$06-15-94$ & 0838 & Culvert & N/A & 0.01 & NA & NA & MM \\
$06-21-94$ & 1643 & Cuivert & 0.67 & 0.01 & 3.67 & 422.85 & PM, WS above culvert \\
$06-27-94$ & 1610 & Culvert & NA & 0.01 & NA & NA & PM, WS above culvert \\
$07-01-94$ & 1517 & Culvert & -0.81 & 0.01 & 2.19 & 421.37 & PM \\
$07-15-94$ & 0915 & Culvert & -2.27 & 0.01 & 0.73 & 419.91 & MM \\
$08-05-94$ & 1630 & Culvert & -1.87 & 0.01 & 1.13 & 420.31 & PM \\
$08-12-94$ & 1554 & Culvert & -1.48 & 0.01 & 1.52 & 420.70 & PM \\
$08-15-94$ & 1050 & Culvert & -1.93 & 0.01 & 1.07 & 420.25 & MM \\
$09-15-94$ & 1140 & Cuivert & NA & 0.01 & NA & NA & MM, dry
\end{tabular}




\begin{tabular}{|c|c|c|c|c|c|c|c|}
\hline $\begin{array}{l}\text { CU5 SLOUG } \\
\text { CULVERT }\end{array}$ & SERV & SITE & & ontinut & & Station ID: & 1551401730 \\
\hline Dafe & Time & Method & MP to WS & Error & LS to WS & WS elevation & Remarks \\
\hline $10-18-94$ & 1640 & Culvert & NA & 0.01 & NA & NA & MM, dry \\
\hline $11-16-94$ & 1415 & Culvert & NA & 0.01 & NA & NA & $M M_{1} d r y$ \\
\hline $12-23-94$ & 1436 & Culvert & NA & 0.01 & NA & NA & $M M, d r y$ \\
\hline $04-18-95$ & 1440 & Culvent & NA & 0.01 & NA & NA & $M M, d r y$ \\
\hline $02-16-95$ & 1525 & Culvert & NA & 0.01 & NA & NA & $\mathrm{MA}_{1} \mathrm{dry}$ \\
\hline $04-15-95$ & 1231 & Culvert & NA & 0.01 & NA & NA & MM, covered in snow \\
\hline $05-16-95$ & 1354 & Culvert & NA & 0.01 & NA & NA & MM, dry \\
\hline $05-31-95$ & 0850 & Culvert & NA & 0.01 & NA & NA & $P M, d r y$ \\
\hline $07-18-95$ & 0900 & Culvert & -1.53 & 0.01 & 1.47 & 420.65 & MM \\
\hline $08-15-95$ & 1354 & Culvert & $\mathrm{NA}$ & 0.01 & NA & NA & MM, dry \\
\hline $09-14-95$ & 1208 & Culvert & -1.97 & 0.01 & 1.03 & 420.21 & $M M$ \\
\hline $10-17-95$ & 1030 & Culvert & NA & 0.01 & $\mathrm{NA}$ & NA & $M M_{x} d r y$ \\
\hline
\end{tabular}




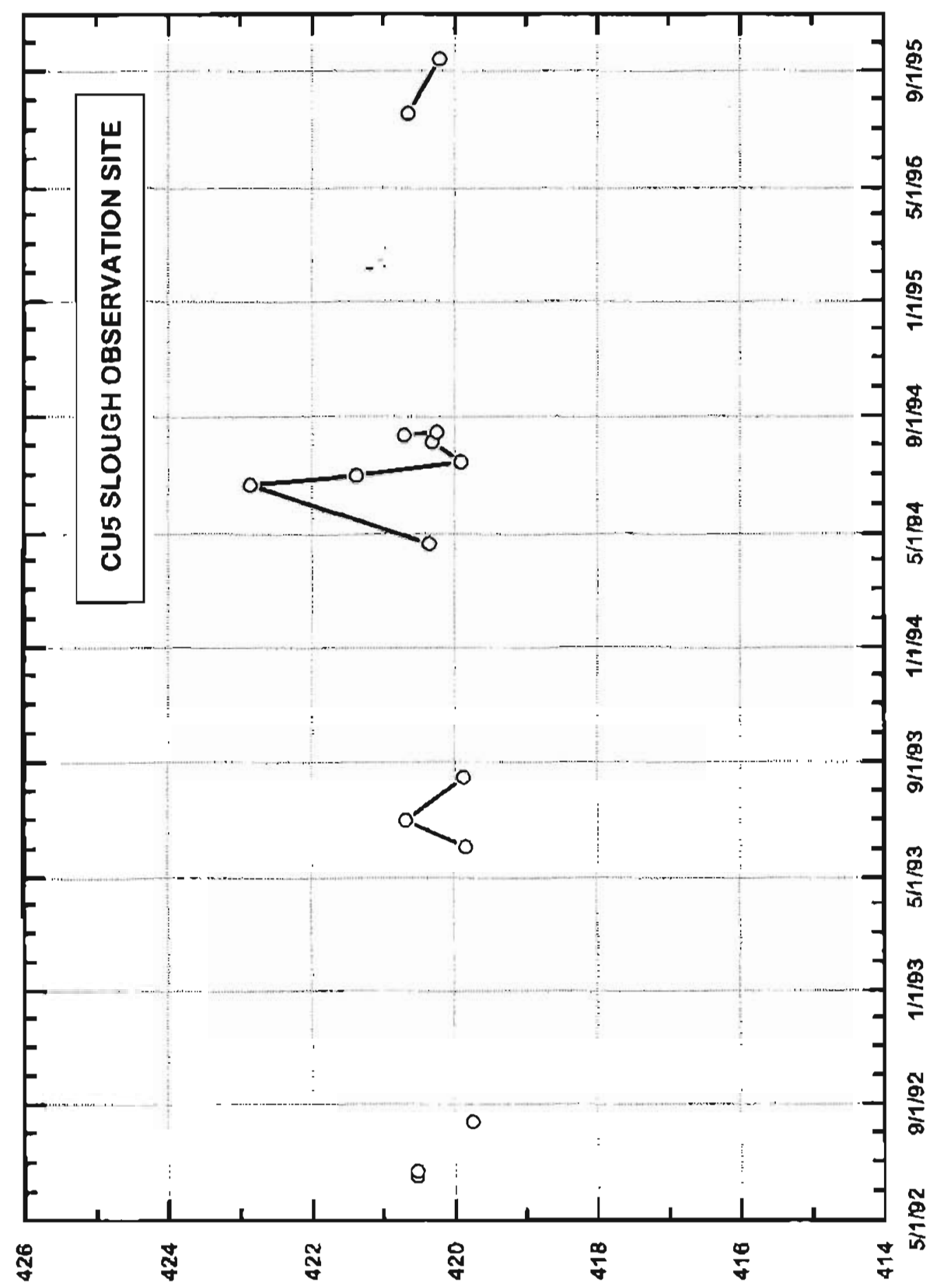

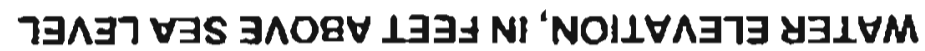




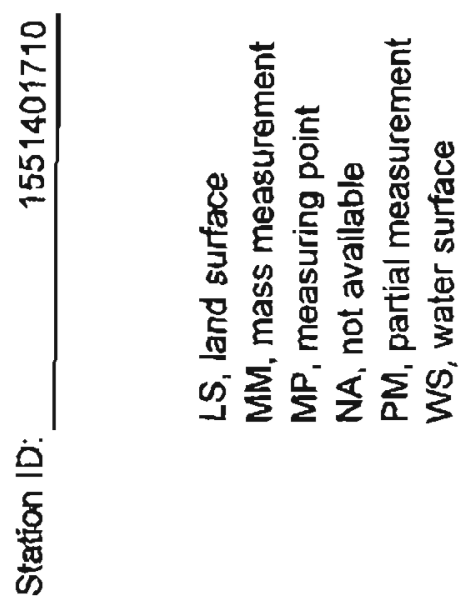

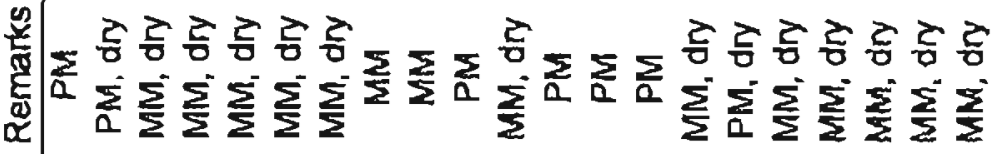

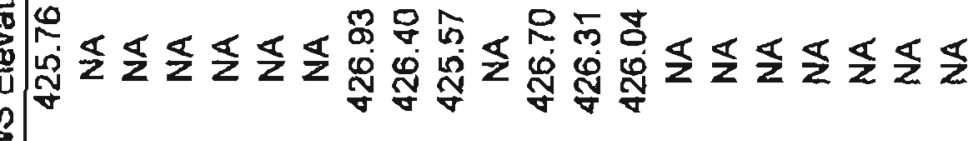

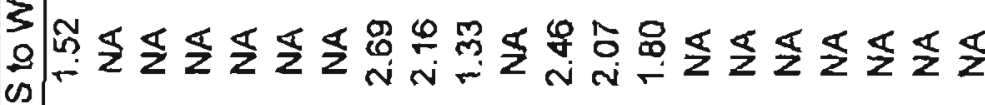
cos

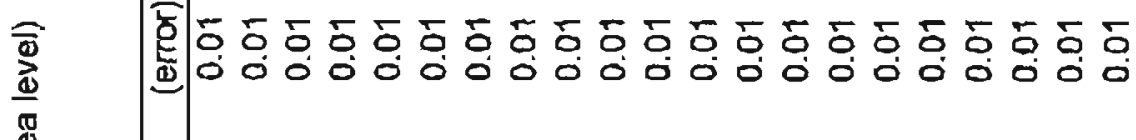

m

象

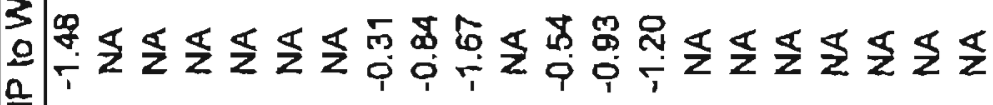

$\frac{2}{2}$

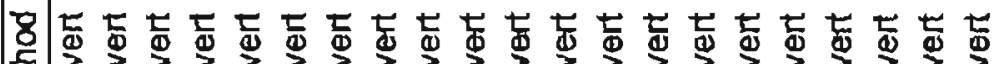

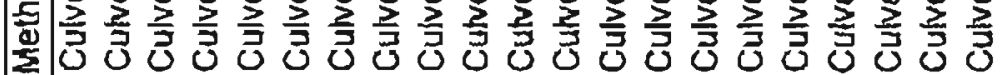

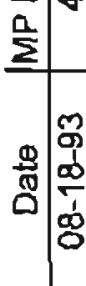

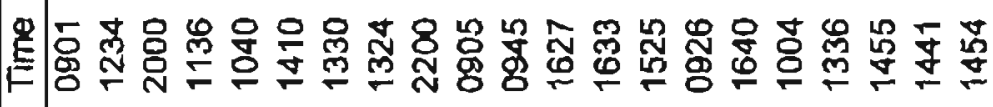

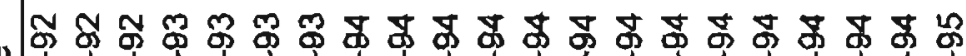

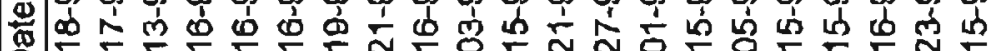

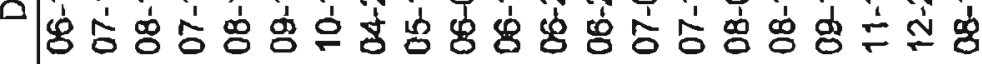




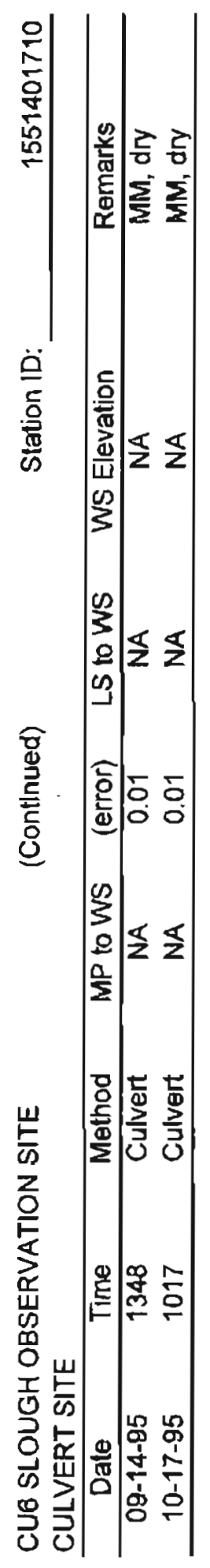




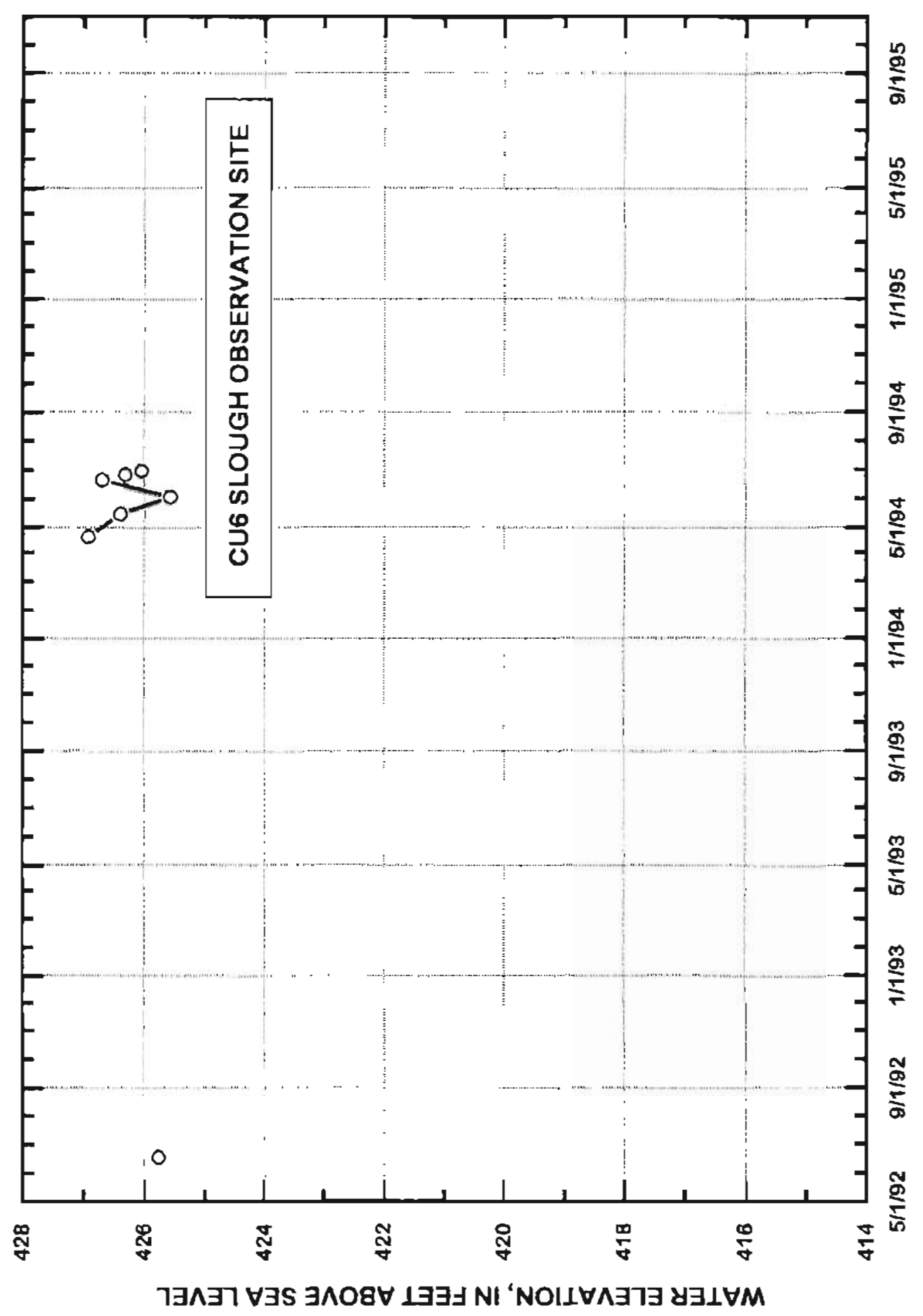




\section{CULVERT SITE}

All measurements in feet

Cuivert diameter.

Land Surtace Datum:

417.91

Datum corractions, refarence survey notes in site folders

\begin{tabular}{c|c} 
Date & MP Elevation (leet above sea level) \\
\hline $07-01-92$ & 420.91
\end{tabular}
LS, land surface $M M_{2}$ mass measurement MP, measuring point NA, not available PM, partiat measurement WS, weter surface

\begin{tabular}{|c|c|c|c|c|c|c|c|}
\hline Date & Time & Method & MP to WS & Error & LS to WS & WS elevation & Remarks \\
\hline $07-17-92$ & 1132 & Culvert & -1.51 & 0.01 & 1.49 & 419.40 & MM \\
\hline $08-13-92$ & 1909 & Culvert & -1.15 & 0.01 & 1.85 & 419.76 & PM \\
\hline $09-14-92$ & 0849 & Culvert & NA & 0.01 & NA & NA & $P M, d r y$ \\
\hline $11-05-92$ & 1030 & Culvert & NA & 0.01 & NA & NA & $P M_{1}$ dry \\
\hline $06-03-93$ & 1129 & Culvert & -1.05 & 0.01 & 1.95 & 419.86 & PM \\
\hline $07-17-93$ & 1317 & Culvert & NA & 0.01 & NA & NA & MM, dry \\
\hline $08-16-93$ & 1101 & Culvert & -1.38 & 0.01 & 1.62 & 419.53 & $\mathrm{MM}$ \\
\hline $10-19-93$ & 1340 & Culvert & $\mathrm{NA}$ & 0.01 & NA & NA & MM, frozen \\
\hline $01-20-94$ & 1335 & Culvert & NA & 0.01 & NA & NA & $M M$, frozen \\
\hline $04-21-94$ & 1425 & Culvert & -0.43 & 0.01 & 2.57 & 420,48 & MM \\
\hline $06-09-94$ & 1709 & Cutvert & $\mathbf{N A}$ & 0.01 & $\mathbf{N A}$ & NA & PM \\
\hline $06-\$ 5-94$ & 0838 & Culvert & $\mathrm{NA}$ & 0.01 & NA & NA & MM \\
\hline $06-21-94$ & 1643 & Culvert & 0.53 & 0.01 & 3.53 & 421.44 & PM, WS above culvert \\
\hline $06-27-94$ & 1812 & Cunert & NA & 0.01 & NA & NA & PM, WS above culvert \\
\hline $07-01-94$ & 1517 & Culvert & -0.50 & 0.01 & 2.50 & 420.41 & PM \\
\hline $07-15-94$ & 0915 & Culvert & -0.96 & 0.01 & 2.04 & 419.95 & MA \\
\hline $08-05-94$ & 1630 & Culvert & -0.58 & 0.01 & 2.42 & 420.33 & PM \\
\hline $08-12-94$ & 1554 & Culvert & -0.17 & 0.01 & 2.83 & 420.74 & PM \\
\hline $08-15-94$ & 1050 & Culvert & -0.58 & 0.01 & 2.42 & 420.33 & $M M$ \\
\hline $09-15-94$ & $1 \uparrow 40$ & Cukert & NA & 0.01 & NA & NA & $\mathrm{MA}, \mathrm{dry}$ \\
\hline $10-18-94$ & 1640 & Culvert & NA & 0.01 & NA & NA & $M M_{1}$ dry \\
\hline $11-16-94$ & 1415 & Culvert & NA & 0.01 & NA & NA & MM, dry \\
\hline
\end{tabular}




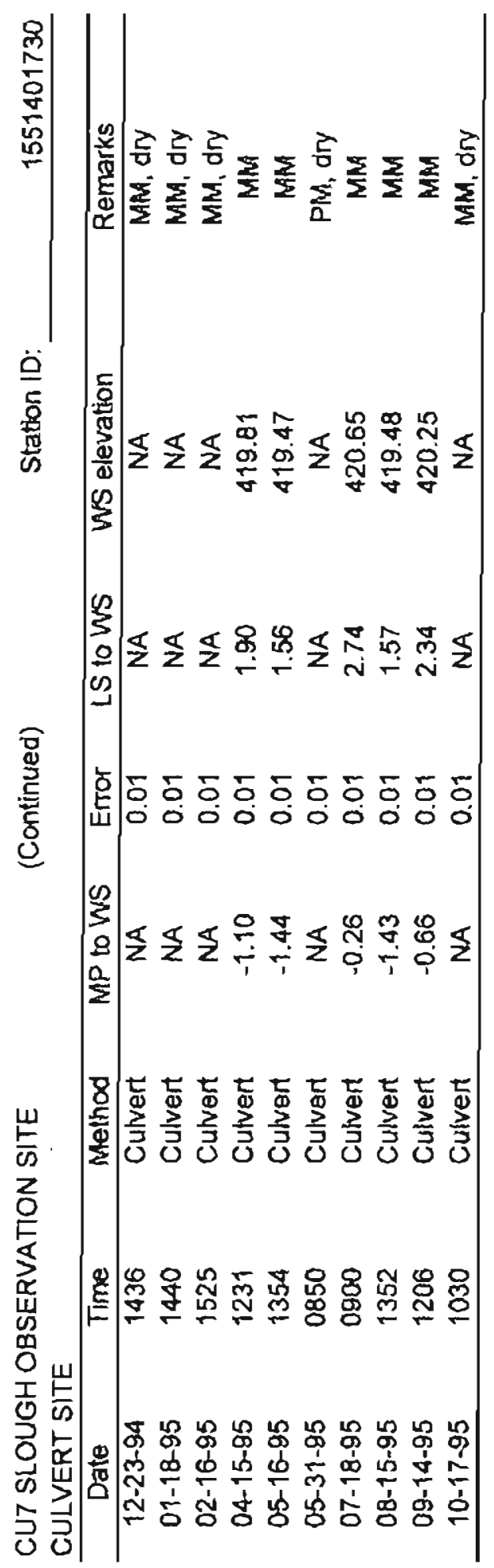




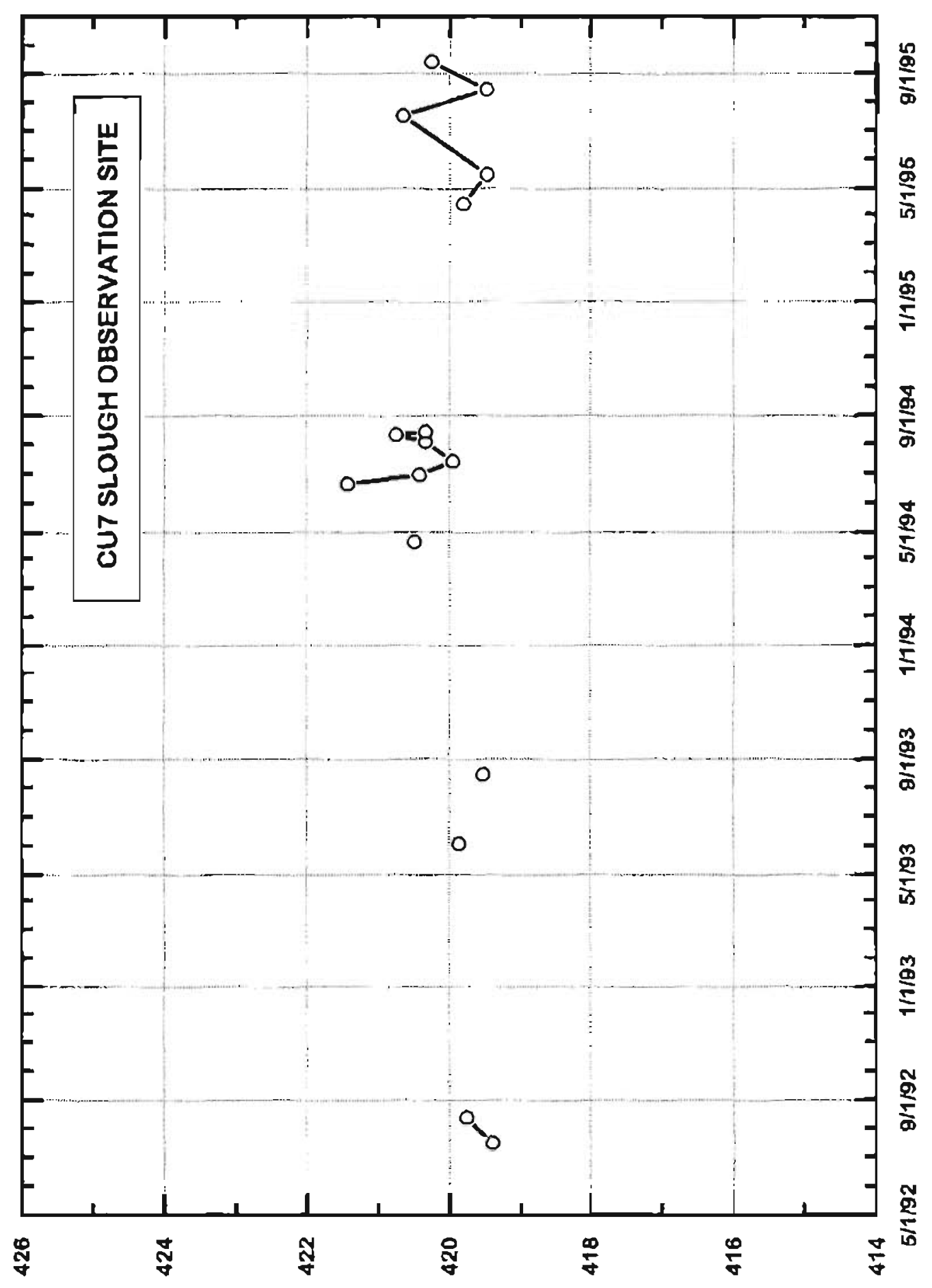

1ヨ^ヨา $\forall \exists S \exists \wedge$ ง 
RB1 SLOUGH OBSERVATION SITE

Station ID: 1551401790

REBAR SITE

All measurements in feet

Rebar stick up: $\quad 1.35$

Land Surface Datum (c16): $\quad 423.33$

Datum corrections, reference survey notes in site folders

S, land surface

AAM, mass measurement

MP, measuring point

NA, not aväiable

$P M_{\text {, partial measurement }}$

\begin{tabular}{c|c} 
Date & MP Elevation (feet above sea level) \\
\hline $07-01-\overline{92}$ & 424.68
\end{tabular}

WS, water surface

\begin{tabular}{|c|c|c|c|c|c|c|c|}
\hline Date & Time & Method & MP to $W S$ & Error & LS to WS & WS elevation & Remarks \\
\hline $06-01-92$ & 2037 & Rebar & 0.20 & 0.01 & 1.55 & 424.88 & $\mathrm{PM}$ \\
\hline $06-02-92$ & 0715 & Rebar & 0.18 & 0.01 & 1.53 & 424.86 & $P M$ \\
\hline $06-02-92$ & 2230 & Rebar & 0.25 & 0.01 & 3.60 & 424.93 & PM \\
\hline $06-04-92$ & 0751 & Rebar & 0.80 & 0.01 & 2.15 & 425.48 & PM \\
\hline $06-16-92$ & 0740 & Rebar & NA & 0.01 & NA & NA & PM, dry \\
\hline $09-14-92$ & 1107 & Rebar & -5.55 & 0.01 & $-4,20$ & 419.13 & $P M$ \\
\hline $11-06-92$ & 1035 & Rebar & MA & 0.01 & NA & NA & $\mathrm{MM}, \mathrm{dry}$ \\
\hline $06-03-93$ & 1455 & Rebar & -5.09 & 0.01 & -3.74 & 419.59 & PM \\
\hline $07-16-93$ & 1333 & Rebar & 0.23 & 0.01 & 1.58 & 424.91 & MA \\
\hline $07-28-93$ & 1754 & Rebar & -3.10 & 0.01 & -1.75 & 421.58 & PNA \\
\hline $08-16-93$ & 1115 & Rebar & -2.88 & 0.01 & -1.53 & 421.80 & MM \\
\hline $10-19-93$ & 1351 & Rebar & NA & 0.01 & NA & NA & MM, dry \\
\hline $06-09-94$ & 1715 & Rebar & $\mathrm{NA}$ & 0.01 & NA & $\mathrm{NA}$ & PM \\
\hline $06-15-94$ & 0842 & Rebar & NA & 0.01 & NA & NA & MN \\
\hline $07-01-94$ & 1508 & Rebar & NA & 0.01 & NA & NA & Under water \\
\hline $07-15-94$ & 0919 & Rebar & -0.22 & 0.01 & -1.13 & 424.46 & MM \\
\hline $08-05-94$ & 1620 & Reber & NA & 0.01 & NA & NA & PM, unable to locate \\
\hline $08-12-94$ & 1600 & Rebar & NA & 0.01 & NA & NA & PM, unable to locate \\
\hline $08-15-94$ & 1045 & Rebar & NA & 0.01 & NA & NA & PM, unable to locate \\
\hline $09-15-94$ & 1133 & Rebar & NA & 0.01 & NA & NA & $P M$, ory \\
\hline $10-18-94$ & 1638 & Rebar & NA & 0.01 & NA & NA & $M M, d r y$ \\
\hline
\end{tabular}


RB I SLOUGH OBSERVATION SITE

(Continued)

Station ID:

1551401790 REBAR SITE

\begin{tabular}{cccccccc}
\hline Date & Time & Method & MP to WS & Error & LS to WS & WS elevation & Remarks \\
\hline $11-16-94$ & 1413 & Rebar & NA & 0.01 & NA & NA & MM, dry \\
$12-23-94$ & 1433 & Rebar & NA & 0.01 & NA & NA & MM, dry \\
$01-18-95$ & 1414 & Rebar & NA & 0.01 & NA & NA & MM, dry \\
$02-16-95$ & 1535 & Rebar & NA & 0.01 & NA & NA & MM, dry \\
$04-15-95$ & 1254 & Rebar & -0.28 & 0.01 & -1.07 & 424.40 & MM \\
$05-16-95$ & 1404 & Rebar & -1.69 & 0.01 & 0.34 & 422.99 & MM \\
$08-15-95$ & 1605 & Rebar & -0.30 & 0.01 & -1.05 & 424.38 & Site disturbed, check MP \\
\hline
\end{tabular}




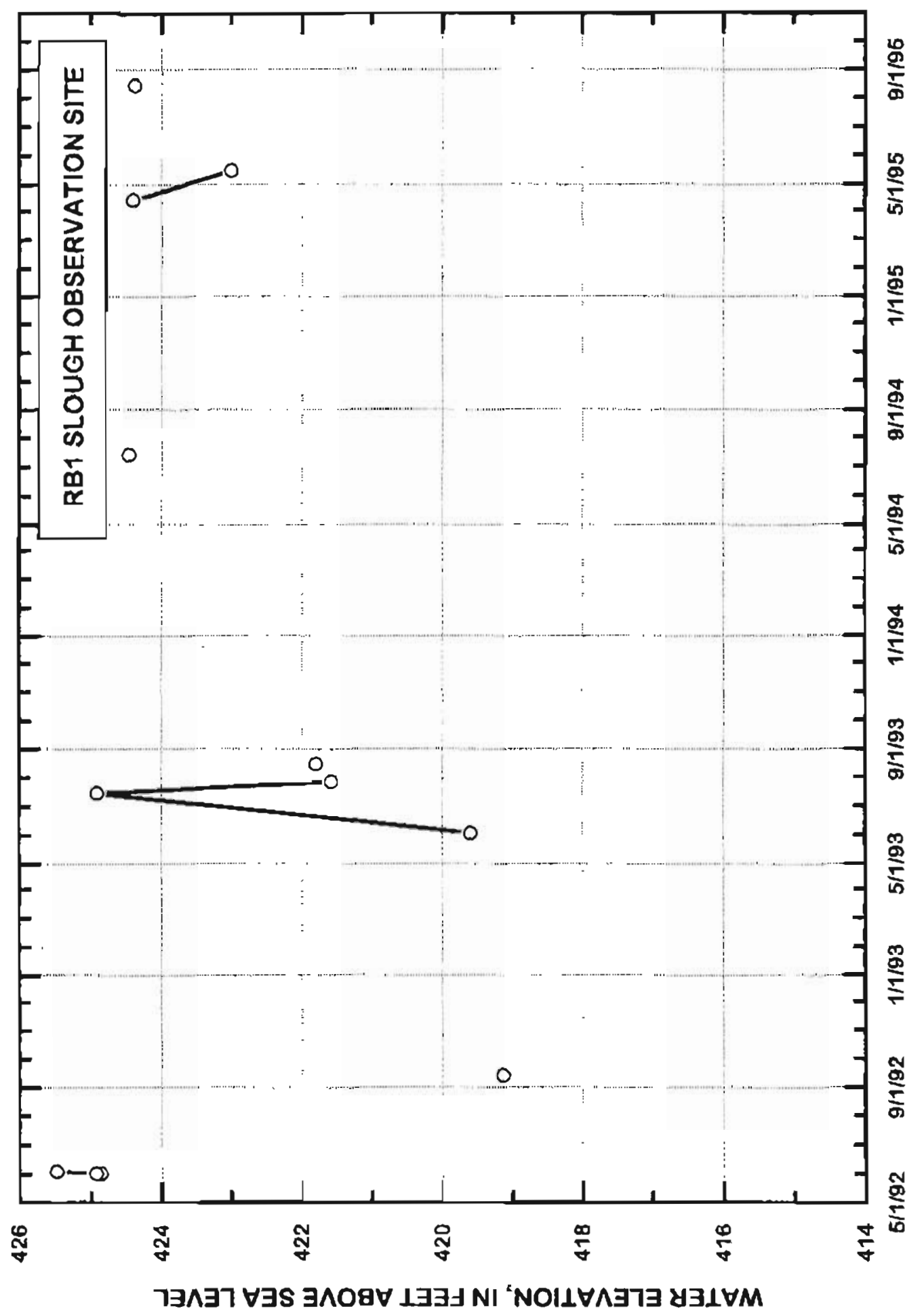


RB2 SLOUGH OBSERVATION SITE

Station ID:

1551401730

All measurements in feet

Rebar stick up: $\quad 1.35$

Land Surface Datum : $\quad \mathbf{4 2 3 . 6 9}$

Datum corrections, reference survey notes in site folders

S, land surface

MM, mass measurement

$M P$, measuring point

NA, not avallable

PM, partial measurement

\begin{tabular}{c|c} 
Date & MP Elevation (feet above sea level) \\
\hline $07-0 \overline{7-92}$ & 425.04
\end{tabular}

WS, water surface

\begin{tabular}{cccccccc}
\hline Date & Sime & Method & MP to WS & Error & LS to WS & WS elevation & Remarks \\
\hline $06-01-92$ & 2113 & Rebar & 0.29 & 0.01 & 1.64 & 425.33 & PM \\
$06-02-92$ & 2148 & Rebar & 0.28 & 0.01 & 1.63 & 425.32 & PM \\
$06-04-92$ & 0807 & Rebar & 0.06 & 0.01 & 1.41 & 425.10 & PM \\
$06-13-92$ & 1813 & Rebar & 0.28 & 0.01 & 1.63 & 425.32 & PM \\
$06-16-92$ & 0845 & Rebar & -0.13 & 0.01 & -1.48 & 424.91 & PM \\
$06-17-92$ & 1017 & Rebar & -0.18 & 0.01 & -1.53 & 424.86 & PM \\
$06-22-92$ & 0900 & Rebar & -0.50 & 0.01 & -1.85 & 424.54 & PM \\
$09-14-92$ & 1131 & Rebar & NA & 0.01 & NA & NA & PM, dry \\
$06-15-94$ & 0937 & Rebar & NA & 0.01 & NA & NA & PMy \\
$06-21-94$ & 1704 & Rebar & NA & 0.01 & NA & NA & PM, dry \\
$07-01-94$ & 1539 & Rebar & NA & 0.01 & NA & NA & MM, dry \\
$08-15-94$ & 1056 & Rebar & NA & 0.01 & NA & NA & MM, dry \\
$09-15-94$ & 1405 & Rebar & NA & 0.01 & NA & NA & MM, skough fillod with H20 \\
$04-15-95$ & 1148 & Rebar & NA & 0.01 & NA & NA
\end{tabular}




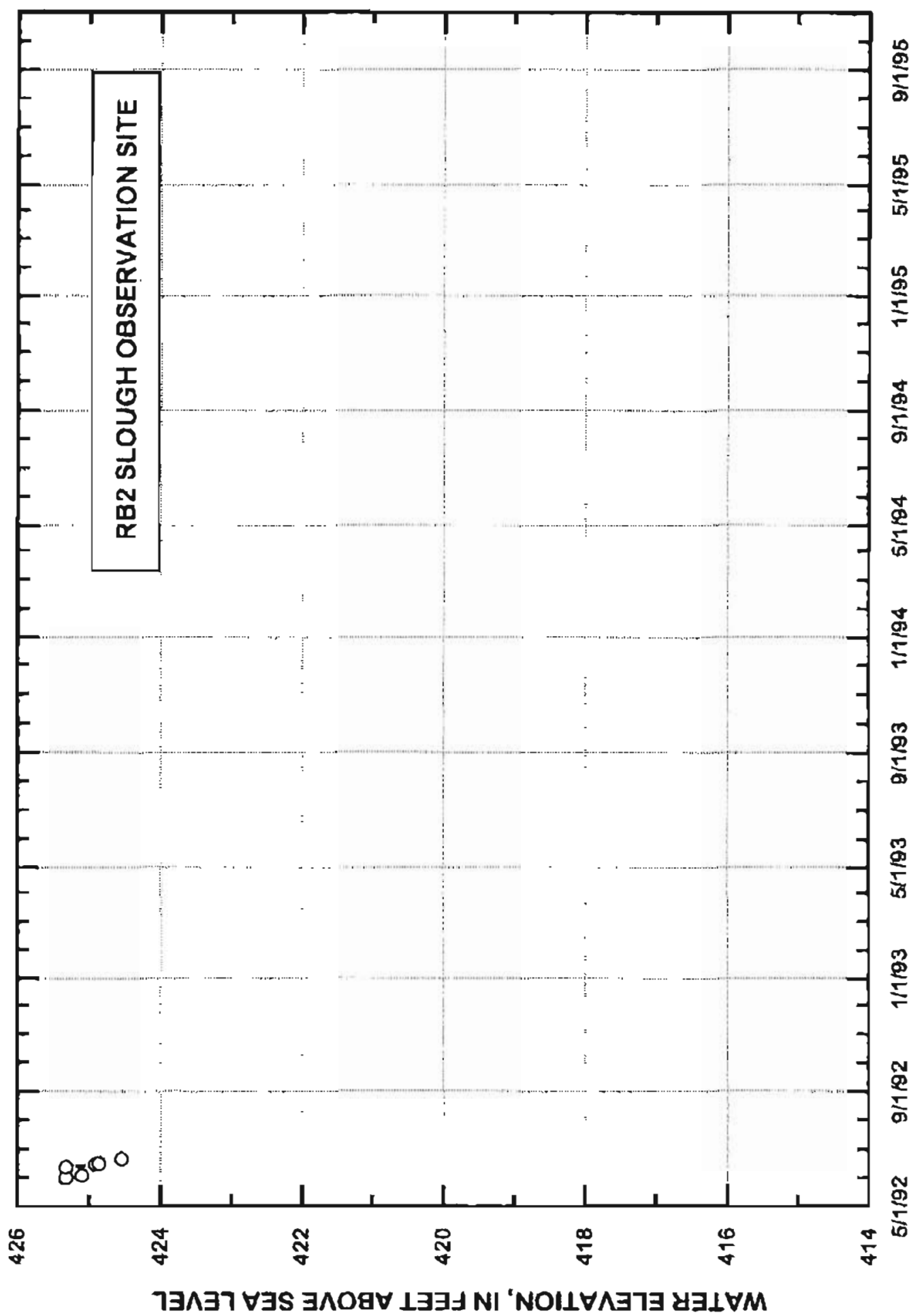


Chena River at University Avenue Bridge

Site ID: 15514016

Wire Weight Gage

All measurements in feet

Datum corrections, reference survey notes in site folders

Land Surface Datum : $\quad \mathbf{4 1 5 . 0 0}$

\begin{tabular}{c|cc} 
Date & CB elevation (feet above sea level) \\
\hline $08-10-92$ & 438.90 & \\
$06-22-93$ & 438.86 & Updated dalum
\end{tabular}

$\mathrm{CB}$, check bar

f/s, foot per second

MM, mass measurement

$P M_{\text {s }}$ partlat measurement

WS, water surface

06-22-93 438.86 Updated dalum

\begin{tabular}{ccccccc}
\hline Date & Time & Check bar & CE to WS & ETor & WS etevation & Remarks \\
\hline $05-07-92$ & 1011 & 38.85 & 17.42 & 0.02 & 417.47 & PM \\
$05-14-92$ & 1031 & 38.85 & 18.85 & 0.02 & 418.90 & MM \\
$05-24-92$ & 1900 & 38.85 & 22.56 & 0.02 & 422.61 & PM, turbid, floating debris \\
$05-26-92$ & 1254 & 38.85 & 24.30 & 0.02 & 424.35 & PM \\
$05-26-92$ & 1400 & 38.85 & 24.33 & 0.02 & 424.38 & PM, moderate debris \\
$05-27-92$ & 0810 & 38.85 & 24.58 & 0.02 & 424.63 & PM \\
$05-29-92$ & 1630 & 38.85 & 25.97 & 0.02 & 426.02 & PM \\
$05-30-92$ & 1030 & 38.85 & 24.97 & 0.02 & 425.02 & PM \\
$05-3+-92$ & 1810 & 38.85 & 24.78 & 0.02 & 424.83 & PM \\
$06-01-92$ & 2155 & 38.85 & 24.51 & 0.02 & 424.56 & PM \\
$06-02-92$ & 0737 & 38.85 & 24.50 & 0.02 & 424.55 & PM \\
$06-02-92$ & 2316 & 38.85 & 24.56 & 0.02 & 424.61 & PM \\
$06-03-92$ & 2322 & 38.85 & 24.89 & 0.02 & 424.94 & PM \\
$06-04-92$ & 1000 & 38.85 & 25.10 & 0.02 & 425.15 & PM \\
$06-08-92$ & 1913 & 38.85 & 24.08 & 0.02 & 424.13 & MM \\
$06-16-92$ & 0830 & 38.85 & 20.28 & 0.02 & 420.33 & PM \\
$06-17-92$ & 0805 & 38.85 & 20.42 & 0.02 & 420.47 & PM \\
$06-22-92$ & 0733 & 38.85 & 20.48 & 0.02 & 420.53 & PM \\
$07-08-92$ & 1400 & 38.85 & 20.24 & 0.02 & 420.29 & MM \\
$07-17-92$ & 1058 & 38.85 & 20.59 & 0.02 & 420.64 & PM \\
$07-24-92$ & 1745 & 38.85 & 20.71 & 0.02 & 420.76 & MM
\end{tabular}




\begin{tabular}{|c|c|c|c|c|c|c|}
\hline \multicolumn{4}{|c|}{$\begin{array}{l}\text { Chena River at University Avenue Bridge } \\
\text { Wire Weight Gage }\end{array}$} & \multirow{2}{*}{$\begin{array}{c}\text { (Continued) } \\
\text { Error }\end{array}$} & Site ID: & 15514016 \\
\hline Date & Time & Check bar & WS & & WS elevation & Remarks \\
\hline $09-14-92$ & 0820 & 38.85 & 18.15 & 0.02 & 418.20 & PM \\
\hline $11-05-92$ & 0930 & 38.85 & 17.23 & 0.02 & 417.28 & $\mathrm{AMM}$, ice \\
\hline $12-15-92$ & 1200 & 38.85 & 17.96 & 0.02 & 418.01 & MM, ice \\
\hline $02-20-93$ & 1333 & 38.85 & 17.41 & 0.02 & $4 \uparrow 7.46$ & $M M, \mathrm{kCe}$ \\
\hline $03-12-93$ & $\{520$ & 38.85 & 17.11 & 0.04 & 417.16 & MM, ice \\
\hline $04-02-93$ & 1004 & 38.85 & 16.97 & 0.04 & 417.02 & PM, ice \\
\hline $04-02-93$ & 1532 & 38.85 & 16.98 & 0.04 & 417.03 & PM, ice \\
\hline $04-02-93$ & 1605 & 38.85 & 16.99 & 0.04 & 417.04 & $P M$, ice \\
\hline $04-09-93$ & 1335 & 38.85 & 17.21 & 0.04 & 417.26 & PM, flowing water \\
\hline $04-12-93$ & 1633 & 38.85 & 17.39 & 0.02 & 417.44 & PM, clear of ice \\
\hline $04-14-93$ & 1408 & 38.85 & 17.48 & 0.02 & 417.53 & PM \\
\hline $04-16-93$ & $\$ 210$ & 38.85 & 17.67 & 0.02 & 417.72 & $M M$ \\
\hline $04-19-93$ & 1745 & 38.85 & 18.24 & 0.02 & 418.29 & PM \\
\hline $04-21-93$ & 1620 & 38.85 & 19.04 & 0.02 & 419.09 & PM \\
\hline $04-23-93$ & 1630 & 38.85 & 20.02 & 0.02 & 420.07 & $\mathrm{PM}$ \\
\hline $04-25-93$ & 0840 & 38.85 & 20.66 & 0.02 & 420.71 & $\mathrm{PM}_{1}$ no jee running, vel., $1 \mathrm{f} / \mathrm{s}$ \\
\hline $05-0+-93$ & 1959 & 38.85 & 21.12 & 0.02 & 421.17 & PM \\
\hline $05-02-93$ & 2150 & 38.85 & 21.18 & 0.02 & 421.23 & $\mathrm{PM}$ \\
\hline $05-03-93$ & 1111 & 38.85 & 21.30 & 0.02 & 421.35 & $P M$ \\
\hline $05-05-93$ & 1205 & 38.85 & 21.15 & 0.02 & 421.20 & $\mathrm{PM}$ \\
\hline $05-08-93$ & 2142 & 38.85 & 21.12 & 0.02 & 421.17 & PM \\
\hline $05-10-93$ & 1355 & 38.85 & 21.72 & 0.02 & 421.77 & PM \\
\hline $05-12-93$ & 1130 & 38.85 & 21.10 & 0.02 & 421.15 & PM \\
\hline $05-14-93$ & 1100 & 38.85 & 20.90 & 0.02 & 420.95 & $\mathrm{PM}$ \\
\hline $05-18-93$ & 1550 & 38.85 & 22.69 & 0.02 & 422.74 & MM \\
\hline $05-24-93$ & 1405 & 38.85 & 19.94 & 0.02 & 419.99 & PM \\
\hline $05-26-93$ & 1715 & 38.85 & 19.77 & 0.02 & 419.82 & PM \\
\hline $05-29-93$ & 1410 & 38.85 & 19.63 & 0.03 & 419.68 & PM \\
\hline $06-02-93$ & 1545 & 38.84 & 19.24 & 0.02 & 419.30 & PM \\
\hline $06-08-93$ & 1405 & 38.85 & 19.07 & 0.02 & 419,12 & PM \\
\hline $06-11-93$ & 1228 & 38.85 & 18.84 & 0.02 & 418.89 & MM \\
\hline $06-15-93$ & 1930 & 38.85 & 18.83 & 0.02 & 418.88 & PM \\
\hline
\end{tabular}



Wire Weight Gage

\begin{tabular}{|c|c|c|c|c|c|c|}
\hline Date & Time & Check bar & Ws & EтTor & WS elevation & Remarks \\
\hline $06-21-93$ & 1045 & 38.85 & 20.76 & 0.02 & 420.81 & $P M$ \\
\hline $06-25-93$ & 1652 & 38.84 & 19.89 & 0.02 & $419.9\}$ & $P A_{1}$ has been surveyed \\
\hline $06-30-93$ & 1935 & 38.85 & 19.57 & 0.02 & 419.58 & $\mathrm{PM}$ \\
\hline $07-02-93$ & 1830 & 38.85 & 20.84 & 0.02 & 420.85 & PM \\
\hline $07-08-93$ & 1447 & 38.85 & 19.29 & 0.02 & 419.30 & PM \\
\hline $07-17-93$ & 1709 & 38.84 & 19.68 & 0.02 & 419.70 & MM \\
\hline $07-21-93$ & 1700 & 38.84 & 20.21 & 0.02 & 420.23 & $P M$ \\
\hline $07-28-93$ & 1802 & 38.84 & 19.17 & 0.02 & 419.19 & $P M$ \\
\hline $08-05-93$ & 1852 & 38.84 & 19.77 & 0.02 & 419.79 & PM \\
\hline $08-11-93$ & $\uparrow 435$ & 38.85 & 20.31 & 0.02 & 420.32 & PM \\
\hline $08-16-93$ & 1134 & 38.85 & 20.35 & 0.02 & 420.36 & MM \\
\hline $08-27-93$ & 1940 & 38.85 & 19.34 & 0.02 & 419,35 & PM \\
\hline $09-08-93$ & 1844 & 38.85 & 20.45 & 0.02 & 420.46 & PM \\
\hline $09-45-93$ & 1600 & 38.85 & 19.47 & 0.02 & 419.48 & MM \\
\hline $09-29-93$ & 1655 & 38.84 & 19.86 & 0.02 & 419.88 & $\mathrm{PM}$ \\
\hline $10-08-93$ & 3602 & 38.85 & 19.35 & 0.02 & 419.36 & PM \\
\hline $10-14-93$ & 1317 & 38.85 & 19.49 & 0.02 & 419.50 & PM \\
\hline $10-19-93$ & 1317 & 38.85 & 19.45 & 0.02 & 419.46 & MM \\
\hline $01-19-94$ & 1315 & 38.85 & 17.93 & 0.02 & 417.94 & MM \\
\hline $02-22-94$ & 1549 & 38.86 & 17.75 & 0.02 & 417.75 & MM \\
\hline $03-31-94$ & 1500 & 38.85 & 17.13 & 0.02 & 417.14 & MM \\
\hline $04-18-94$ & 2019 & 38.85 & 17.96 & 0.02 & 417.97 & MM \\
\hline $04-21-94$ & 1538 & 38.85 & 17.10 & 0.02 & 417.11 & MM \\
\hline $04-25-94$ & 1245 & 38.85 & 18.51 & 0.02 & 418.52 & $\mathrm{PM}$ \\
\hline $04-28-94$ & 1215 & 38.85 & 21.57 & 0.02 & 421.58 & $\mathrm{PM}$ \\
\hline $05-03-94$ & 1557 & 38.85 & 19.74 & 0.02 & 419.75 & MM \\
\hline $05-10-94$ & 1455 & 38.85 & 18.74 & 0.02 & 418.75 & PM \\
\hline $05-20-94$ & 1132 & 38.85 & 48.27 & 0.02 & 418.28 & PM \\
\hline $05-23-94$ & 1628 & 38.85 & 18.19 & 0.02 & 418.20 & PM \\
\hline $05-27-94$ & 0928 & 38.85 & 18.20 & 0.02 & 418.21 & PM \\
\hline $05-31-94$ & 1005 & 38.85 & 17.92 & 0.02 & 417.93 & PM \\
\hline $06-03-94$ & 1215 & 38.85 & 17.80 & 0.02 & 417.81 & PM \\
\hline
\end{tabular}




\begin{tabular}{|c|c|c|c|c|c|c|c|}
\hline & \multicolumn{4}{|c|}{$\begin{array}{l}\text { Chera River at University Avenue Bridge } \\
\text { Wire Weight Gage }\end{array}$} & (Continued) & Site ID: & 15514016 \\
\hline & Date & Time & Check bar & WS & Error & WS elevation & Remarks \\
\hline & $06-06-94$ & 1519 & 38.85 & 17.89 & 0.02 & 417.90 & PM \\
\hline & $06-09-94$ & 1545 & 38.85 & 18.32 & 0.02 & 418.33 & $\mathrm{PM}$ \\
\hline & $06-10-94$ & 1606 & 38.85 & 18.30 & 0.02 & 418.31 & $\mathrm{PM}$ \\
\hline & $06-24 \sim 94$ & 1127 & 38.85 & 24.28 & 0.02 & 424.29 & $\mathrm{PM}$ \\
\hline & $06-27-94$ & 1612 & 38.85 & 24.14 & 0.02 & 424.15 & $\mathrm{PM}$ \\
\hline & $06-29-94$ & 1601 & 38.85 & 23.96 & 0.02 & 423.97 & $\mathrm{PM}$ \\
\hline & $07-06-94$ & 1705 & 38.85 & 20.65 & 0.02 & 420.66 & PM \\
\hline & $07-08-94$ & 1514 & 38.85 & 20.31 & 0,02 & 420.32 & PM \\
\hline & $07-15-94$ & 0738 & 38.85 & 19.66 & 0.02 & 419.67 & MM \\
\hline & $07-19-94$ & 1703 & 38.85 & 19.90 & 0.02 & 419.91 & $\mathrm{PM}$ \\
\hline & $07-21-94$ & 1600 & 38.85 & 19.81 & 0.02 & 419.82 & PM \\
\hline & $07-27-94$ & 1234 & 38.85 & 19.47 & 0.02 & 419.48 & PM \\
\hline & $07-29-94$ & 1115 & 38.85 & 20.11 & 0.02 & 420.12 & PM \\
\hline & $08-02-94$ & 1550 & 38.85 & 20.82 & 0.02 & 420.83 & PM \\
\hline & $08-04-94$ & 1627 & 38.85 & 20.54 & 0.02 & 420.55 & PM \\
\hline & $08-09-94$ & 1558 & 38.85 & 20.62 & 0.02 & 420.63 & $\mathrm{PM}$ \\
\hline$\xi$ & $08-11-94$ & 1054 & 38.85 & 20.68 & 0.02 & 420.69 & PM \\
\hline$\frac{2}{4}$ & $08-15-94$ & 0910 & 38.85 & 20.28 & 0.02 & 420.29 & MM \\
\hline 勇 & $08-19-94$ & 1544 & 38.85 & 20.25 & 0.02 & 420.26 & PM \\
\hline 严 & $08-23-94$ & 1502 & 38.85 & 19.42 & 0.02 & 419.43 & PM \\
\hline 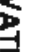 & $10-19-94$ & 1225 & 38.85 & 17.48 & 0.02 & 417.49 & MM \\
\hline$\frac{\partial}{2}$ & $12-23-94$ & 1302 & 38.85 & 17.75 & 0.02 & 417.76 & MM \\
\hline$\overline{5}$ & $01-19-95$ & 1200 & 38.84 & 17.36 & 0.02 & 417.38 & MM \\
\hline & $03-22-95$ & 1025 & 38.85 & 16.81 & 0.02 & 416.82 & MM \\
\hline & $04-14-95$ & 0958 & 38.86 & 16.93 & 0.02 & 416.93 & MM \\
\hline 宓 & $04-23-96$ & 1059 & 38.85 & 18.36 & 0.02 & 418.37 & $P M$ \\
\hline$\underline{x}$ & $04-25-95$ & 1140 & 38.85 & 19.52 & 0.02 & 419.53 & PM \\
\hline 8 & $04-27-95$ & 1326 & 38.85 & 19.90 & 0.02 & 419.91 & $P M$ \\
\hline है & $05-0 t-95$ & $\{225$ & 38.85 & 19.75 & 0.02 & $4\{9.76$ & $\mathrm{PM}$ \\
\hline 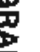 & $05-05-95$ & 1145 & 38.85 & 21.36 & 0.02 & 421.37 & $\mathrm{PM}$ \\
\hline & $05-10-95$ & 1427 & 38.85 & 19.72 & 0.02 & 418.73 & $P M$ \\
\hline & $05-12-95$ & 1212 & 38.85 & 19.68 & 0.02 & 419.69 & PM \\
\hline
\end{tabular}



Wire Weight Gage

\begin{tabular}{ccccccc}
\hline Date & Time & Check bar & WS & Eror & WS elevation & Remarks \\
\hline $05-16-95$ & 1536 & 38.85 & 19.46 & 0.02 & 419.47 & MM \\
$05-18-95$ & 1126 & 38.85 & 19.08 & 0.02 & 419.09 & PM \\
$05-22-95$ & 1404 & 38.85 & 18.80 & 0.02 & 418.81 & PM \\
$06-05-95$ & 1310 & 38.85 & 18.77 & 0.02 & 418.78 & PM \\
$06-08-95$ & 1024 & 38.85 & 18.37 & 0.02 & 418.38 & PM \\
$06-12-95$ & 1328 & 38.85 & 18.19 & 0.02 & 418.20 & PM \\
$06-15-95$ & 1045 & 38.85 & 18.15 & 0.02 & 418.16 & MM \\
$06-20-95$ & 1520 & 38.85 & 18.32 & 0.02 & 418.33 & PM \\
$06-26-95$ & 1625 & 38.85 & 21.86 & 0.02 & 421.87 & PM \\
$06-28-95$ & 1235 & 38.85 & 24.08 & 0.02 & 424.09 & PM \\
$06-30-95$ & 1245 & 38.85 & 22.99 & 0.02 & 423.00 & PM \\
$07-03-95$ & 1125 & 38.85 & 21.07 & 0.02 & 421.08 & PM \\
$07-10-95$ & 1046 & 38.85 & 20.51 & 0.02 & 420.52 & PM \\
$07-13-95$ & 1244 & 38.85 & 20.37 & 0.02 & 420.38 & MM \\
$07-17-95$ & 1511 & 38.85 & 21.25 & 0.02 & 421.26 & PM \\
$07-21-95$ & 1514 & 38.85 & 20.39 & 0.02 & 420.40 & PM \\
$07-25-95$ & 0937 & 38.85 & 20.50 & 0.02 & 420.51 & PM \\
$07-27-95$ & 1018 & 38.85 & 20.11 & 0.02 & 420.12 & PM \\
$08-01-95$ & 1319 & 38.85 & 19.24 & 0.02 & 419.25 & PM \\
$08-09-95$ & 1023 & 38.85 & 19.61 & 0.02 & 419.62 & MM \\
$08-15-95$ & 1603 & 38.85 & 19.87 & 0.02 & 419.88 & PM \\
$08-21-95$ & 1300 & 38.85 & 20.17 & 0.02 & 420.18 & MM \\
$09-15-95$ & 1355 & 38.85 & 20.90 & 0.02 & 420.91 & MM \\
$10-17-95$ & 1216 & 38.85 & 18.64 & 0.02 & 418.65 & \\
\hline & & & & & &
\end{tabular}




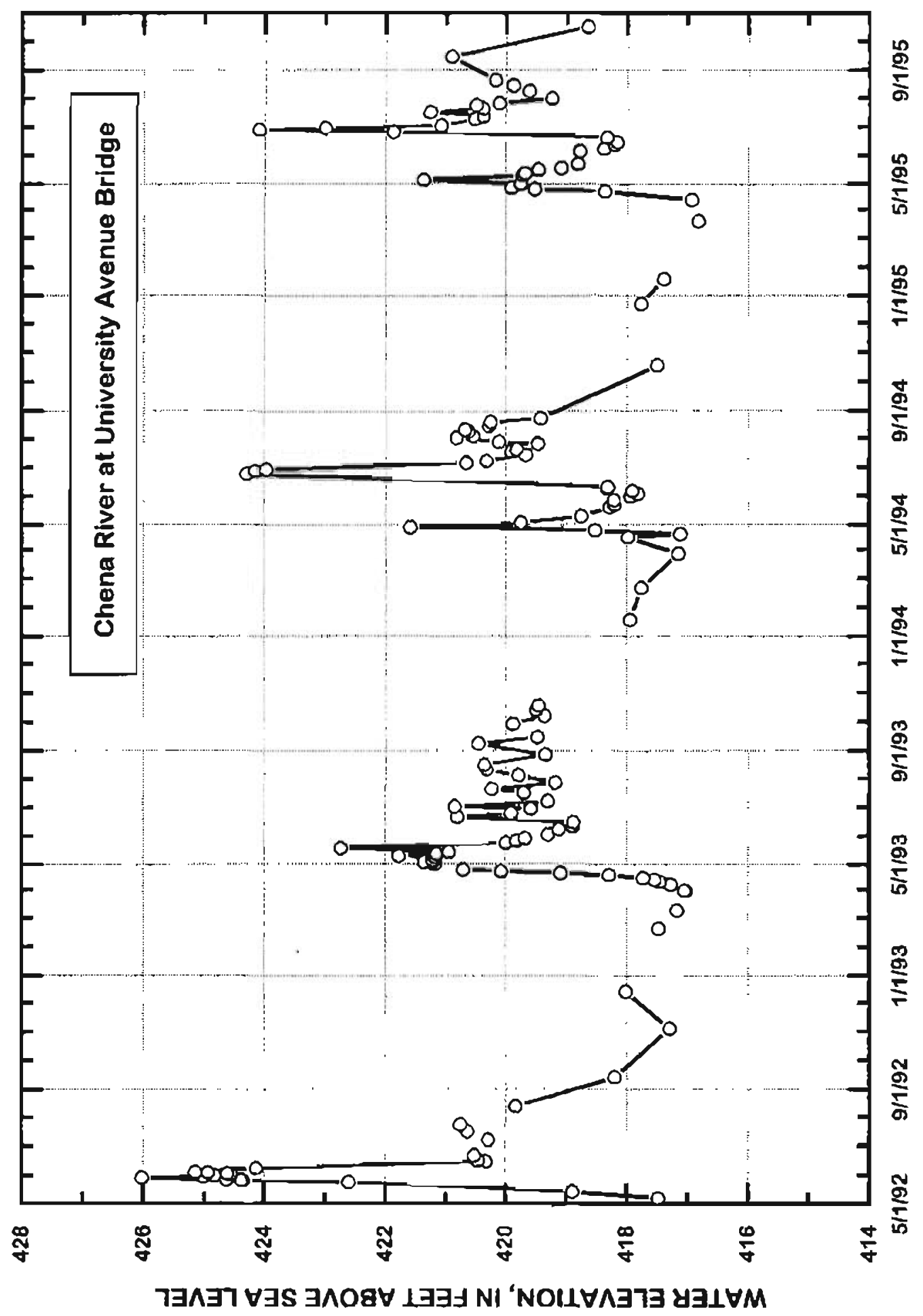


Chena River at Parks Highway Bridge

Site ID: 15514018

Wire Weight Gage

All measurements in feet

Datum corrections, reference survey notes in site folders

Land Surface Datum: 410.00

\begin{tabular}{c|c} 
Date & CB elevation (foet above sea level) \\
\hline $08-05-92$ & 448.99
\end{tabular}

CB, check bar

MM, mass measurement

NA, not available

PM, partial measurement

WS, water sutace

\begin{tabular}{|c|c|c|c|c|c|c|}
\hline Date & Time & Check bar & CB to WS & Error & WS etevation & Remarks \\
\hline $05-19-92$ & 1630 & 90.89 & 57.20 & 0.02 & 415.30 & PM, installed \\
\hline $05-2+-92$ & 0846 & 90.91 & 57.92 & 0.02 & 416.00 & PN, installed backing plate \\
\hline $05-24-92$ & 0800 & 90.90 & 62.48 & 0.02 & 420.57 & $\mathrm{PM}$ \\
\hline $05-24-92$ & 1830 & 90.90 & 62.48 & 0.02 & 420.57 & PM \\
\hline $05-26-92$ & 0641 & 90.90 & 64.37 & 0.02 & 422.46 & PM \\
\hline $05-26-92$ & 1240 & 90.90 & 64.38 & 0.03 & 422.47 & PM \\
\hline $05-27-92$ & 0724 & 90.90 & 64.73 & 0.02 & 422.82 & PM, snowmelt rise \\
\hline $05-29-92$ & 1611 & 90.91 & 65.20 & 0.02 & 423.28 & $\mathbf{P M}$ \\
\hline $05-30-92$ & 1050 & 90.91 & 65.25 & 0.02 & 423.33 & PM \\
\hline $06-01-92$ & 2016 & 90.91 & 64.87 & 0.02 & 422.95 & PM, after heavy rains \\
\hline $06-02-92$ & 0704 & 90.91 & 64.84 & 0.02 & 422.92 & $P M$ \\
\hline $06-02-92$ & 2240 & 90.91 & 64.94 & 0.02 & 423.02 & PM \\
\hline $06-04-92$ & 0740 & 90.91 & 65.48 & 0.02 & 423.56 & $P M$ \\
\hline $06-16-92$ & 0735 & 90.91 & 60.88 & 0.02 & 418.96 & MM \\
\hline $06-17-92$ & 1006 & 90.91 & 61.30 & 0.02 & 419.38 & $\mathbf{M M}$ \\
\hline $06-22-92$ & 0833 & 90.91 & 61.25 & 0.02 & 419.33 & PM \\
\hline $07-17-92$ & 1150 & 90.91 & 62,25 & 0.02 & 420.33 & $M M$ \\
\hline $07-21-92$ & 1830 & 90.90 & 62.65 & 0.02 & 420.74 & PM \\
\hline $08-13-92$ & 0747 & 90.91 & 61.43 & 0.02 & 419.51 & MM \\
\hline $09-14-92$ & 1115 & 90.90 & 57.63 & 0.02 & 415.72 & PM \\
\hline $11-05-92$ & 1055 & 90.90 & 57.60 & 0.10 & 415.69 & $M M$, ice \\
\hline $02-20-93$ & 1500 & 90.86 & 58.06 & 0.04 & 416.19 & $M M$, ice \\
\hline $03-12-93$ & 1600 & 90.85 & 58.89 & 0.02 & 416.83 & $M M$, ice \\
\hline
\end{tabular}




\begin{tabular}{|c|c|c|c|c|c|c|c|}
\hline & $\begin{array}{l}\text { Chena Rive } \\
\text { Wire Weigh }\end{array}$ & Parks H & way Bridge & & Continued) & Site ID & 15514018 \\
\hline & Date & Time & Check bar & ws & Error & WS elevation & Remarks \\
\hline & $04-02-93$ & 1035 & 90.86 & 58.45 & 0.02 & 416.58 & PMt, ice \\
\hline & $04-02-93$ & 1540 & 90.86 & 58.48 & 0.02 & 416.61 & PM, ice \\
\hline & $04-09-93$ & 1353 & 90.88 & 58.78 & 0.02 & 416.89 & PM, flowing water \\
\hline & $04-12-93$ & 1620 & 90.88 & 58.96 & 0.02 & 417.07 & PM, rough water surface \\
\hline & $04-14-93$ & $\$ 420$ & 90.88 & $5 B .86$ & 0.02 & 416.97 & PM, open water \\
\hline & $04-16-93$ & 1150 & 90.88 & 59.25 & 0.02 & 417.36 & MM, open water \\
\hline & $04-19-93$ & 1735 & 90.88 & 59.69 & 0.02 & 417.80 & PM \\
\hline & $04-21-93$ & 1612 & 90.88 & 60.22 & 0.03 & 418.33 & Rough water surface \\
\hline & $04-23-93$ & 1615 & 90.88 & 61.25 & 0.03 & 419.36 & Rough water surface \\
\hline & $05-01-93$ & 1927 & 90.86 & 61.15 & 0.02 & 419.28 & $P M$ \\
\hline & $05-02-93$ & 2214 & 90.85 & 61.39 & 0.02 & 419.53 & $P M$ \\
\hline & $05-03-93$ & 1103 & 80.86 & 61.34 & 0.02 & 419.47 & $P M$ \\
\hline & $05-05-93$ & 1158 & 90.86 & 61.24 & 0.02 & 419.37 & Rough water surface \\
\hline & $05-08-93$ & 2112 & 90.86 & 61.14 & 0.02 & 419.27 & $\mathrm{PM}$ \\
\hline & $05-14-93$ & 1020 & 90.90 & 60.84 & 0.02 & 418.93 & $P M$ \\
\hline & $05-18-93$ & $\{542$ & 90.90 & 62.91 & 0.02 & 421.00 & MM \\
\hline 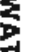 & $05-24-93$ & 1357 & 90.90 & 60.24 & 0.02 & 418.33 & PM \\
\hline 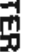 & $05-26-93$ & 1700 & 90.90 & 60.19 & 0.02 & 418.28 & $\mathrm{PM}$ \\
\hline In & $05-29-93$ & 1400 & 90.90 & 59.02 & 0.02 & 417.11 & $\mathrm{PM}$ \\
\hline$\leq$ & $06-02-93$ & 1536 & 90.90 & 59.72 & 0.02 & 417.81 & PM \\
\hline$y$ & $06-08-93$ & 1355 & 90.89 & 59.03 & 0.02 & 417.13 & PM \\
\hline 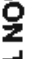 & $06-11-93$ & 1218 & 90.90 & 59.66 & 0.02 & 417.75 & MM \\
\hline 量 & $06-14-93$ & 2000 & 90.90 & 59.57 & 0.02 & 417.66 & PM \\
\hline 㟧 & $06-15-93$ & 1940 & 90.90 & 59.50 & 0.02 & 417.59 & PM \\
\hline 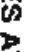 & $06-21-93$ & 1043 & 90.90 & 61.88 & 0.02 & 419.97 & $P M$ \\
\hline z & $06-25-93$ & 1646 & 90.90 & 61.18 & 0.02 & 419.27 & $P M$ \\
\hline I & $06-30-93$ & 1925 & 90.90 & 60.06 & 0.02 & 418.15 & PM \\
\hline & $07-02-93$ & 1800 & 90.90 & 62.50 & 0.02 & 420.59 & PM \\
\hline 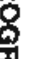 & $07-09-93$ & 0902 & 90.90 & 60.78 & 0.02 & 418.87 & PM \\
\hline W & $07-16-93$ & 1337 & 90.90 & 60.10 & 0.02 & 418.19 & $M M$ \\
\hline & $07-21-93$ & 1650 & 90.90 & 61.01 & 0.02 & 419.10 & $\mathrm{PM}$ \\
\hline & $07-28-93$ & 1747 & 90.90 & 61.51 & 0.02 & 419.60 & PMA \\
\hline
\end{tabular}




\begin{tabular}{|c|c|c|c|c|c|c|}
\hline Date & Time & Check bar & WS & ErTor & WS elevation & Remarks \\
\hline $08-05-93$ & 1830 & 90.90 & 61.47 & 0.02 & 419.56 & $\overline{P M}$ \\
\hline $08-11-93$ & 1423 & 90.90 & 61.73 & 0.02 & 419.82 & PM \\
\hline $08-16-93$ & 1110 & 90.90 & 61.98 & 0.02 & 420.07 & MM \\
\hline $08-27-93$ & 1933 & 80.90 & 59.55 & 0.02 & 417.64 & PM \\
\hline $09-08-93$ & 0852 & 90.90 & 61.73 & 0.02 & 419.82 & PM \\
\hline $09-16-93$ & 1553 & 90.90 & 59.83 & 0.02 & 417.92 & MM \\
\hline $09-29-93$ & 1645 & 90.90 & 59.53 & 0.02 & 417.62 & Cable needs rewinding \\
\hline $10-14-93$ & 1310 & 90.89 & 59.00 & 0.02 & 417.10 & $P M$ \\
\hline $10-19-83$ & 1345 & 90.89 & 58.92 & 0.02 & 417.02 & MM \\
\hline $11-16-93$ & 1300 & 90.90 & NA & 0.02 & NA & MM, Frozen \\
\hline $11-18-93$ & 1300 & 90.90 & 59.33 & 0.02 & 417.42 & MM \\
\hline $12-02-93$ & 1210 & 90.90 & 59.25 & 0.02 & 417.34 & PM \\
\hline $12-22-93$ & 1147 & 90.90 & 58.43 & 0.02 & 416.52 & $\mathrm{MM}$ \\
\hline $01-19-94$ & 1355 & 90.90 & 57.82 & 0.02 & 415.91 & MM \\
\hline $02-22-94$ & 1610 & 90.91 & 58.41 & 0.02 & 416.49 & MM \\
\hline $04-21-94$ & 1630 & 90.90 & 57.94 & 0.02 & 416.03 & MM \\
\hline $04-25-94$ & 1255 & 90.89 & 59.87 & 0.02 & 417.97 & PMA \\
\hline $04-28-94$ & 1227 & 90.89 & 62.78 & 0.03 & 420.88 & PM \\
\hline $05-03-94$ & 1546 & 90.90 & 59.63 & 0.03 & 417.72 & PM \\
\hline $05-10-94$ & 1507 & 90.90 & 57.03 & 0.02 & 415.12 & PM \\
\hline $05-13-94$ & 1135 & 90.90 & 58.14 & 0.02 & 416.23 & $\mathrm{MM}$ \\
\hline $05-20-94$ & 0943 & 90.90 & 57.57 & 0.02 & 415.66 & PM \\
\hline $05-23-94$ & 1700 & 90.90 & 57.43 & 0.02 & 415.52 & PM \\
\hline $05-27-94$ & 0923 & 90.89 & 57.44 & 0.02 & 415.54 & PM \\
\hline $05-31-94$ & 1019 & 90.90 & 57.38 & 0.02 & 415.47 & PMA \\
\hline $06-03-94$ & 1054 & 90.90 & 57.14 & 0.02 & 415.23 & PAA \\
\hline $06-06-94$ & 1528 & 90.90 & 57.54 & 0.02 & 415.63 & PM \\
\hline $06-09-94$ & 1523 & 90.91 & 58.48 & 0.02 & 416.56 & $P M$ \\
\hline $06-10-94$ & 1434 & 90.89 & 58.34 & 0.02 & 416.44 & PM \\
\hline $06-13-94$ & 1056 & 80.89 & 58.40 & 0.02 & 416.50 & PM \\
\hline $06-15-94$ & 1445 & 90.89 & 59.15 & 0.02 & 417.25 & $\mathrm{MM}$ \\
\hline $06-17-94$ & 1522 & 90.90 & 59.98 & 0.02 & 418.07 & PM \\
\hline
\end{tabular}




\begin{tabular}{|c|c|c|c|c|c|c|}
\hline \multicolumn{3}{|c|}{$\begin{array}{l}\text { Chena River at Parks Highway Bridge } \\
\text { Wre Weight Gage }\end{array}$} & & ontinued) & Site ID: & 15514018 \\
\hline Date & Tiste & Check bar & WS & Error & WS elevation & Remarks \\
\hline $06-20-94$ & 1715 & 90.90 & 61.79 & 0.02 & 419.88 & $P M$ \\
\hline $06-22-94$ & 0911 & 90.90 & 64.24 & 0.02 & 422.33 & PM \\
\hline $06-22-94$ & 1158 & 90.90 & 64.41 & 0.02 & 422,50 & PM \\
\hline $06-24-94$ & 0903 & 90.90 & 64.75 & 0.02 & 422.84 & PM \\
\hline $06-24-94$ & 1151 & 90.90 & 64.73 & 0.02 & 422.82 & PM \\
\hline $06-27-94$ & 1623 & 90.90 & 64.61 & 0.02 & 422.70 & PM \\
\hline $06-29-94$ & $\{613$ & 90.90 & 64.21 & 0.02 & 422.30 & PM \\
\hline $07-06-94$ & 1715 & 90.89 & 61.20 & 0.02 & 419.30 & PM \\
\hline $07-08-94$ & $\$ 527$ & 90.89 & 60.93 & 0.02 & 419.03 & PM \\
\hline $07-15-94$ & 0750 & 90.90 & 60.82 & 0.02 & 418.91 & $M M$ \\
\hline $07-19-94$ & $\{703$ & 90.90 & 61.24 & 0.02 & 419.33 & PM \\
\hline $07-21-94$ & 1613 & 90.89 & 60.06 & 0.02 & 418.16 & PM \\
\hline $07-27-94$ & 1256 & 90.90 & 60.81 & 0.02 & 418.90 & PM \\
\hline $07-29-94$ & 1127 & 90.90 & 61.45 & 0.02 & 419.54 & PM \\
\hline $08-02-94$ & 1602 & 90.89 & 61.82 & 0.02 & 419.92 & PM \\
\hline $08-04-94$ & 1645 & 90.89 & 62.21 & 0.02 & 420.31 & PM \\
\hline $08-09-94$ & 1611 & 90.89 & 62.35 & 0.02 & 420.45 & $P M$ \\
\hline $08-1\}-94$ & 1110 & 90.89 & 62.44 & 0.02 & 420.54 & PM \\
\hline $08-15-94$ & 0855 & 90.90 & 61.96 & 0.02 & 420.05 & $M M$ \\
\hline $08-19-94$ & 1557 & 90.90 & 61.98 & 0.02 & 420.07 & $\mathrm{PM}$ \\
\hline $08-23-94$ & 1515 & 90.90 & 61.00 & 0.02 & 419.09 & PM \\
\hline $08-26-94$ & $\{607$ & 90.90 & 59.90 & 0.02 & 417.99 & PM \\
\hline $09-15-94$ & 1204 & 90.89 & 57.83 & 0.02 & 415.93 & MM \\
\hline $09-22-94$ & 1007 & 90.90 & 57.41 & 0.02 & 415.50 & PM \\
\hline $10-03-94$ & 1104 & 90,90 & 56.87 & 0.02 & 414.96 & $\mathrm{PM}$ \\
\hline $10-19-94$ & 1245 & 90.90 & 57.10 & 0.03 & $4 \uparrow 5.19$ & MM \\
\hline $11-16-94$ & 1140 & 90.90 & 57.54 & 0.02 & 415.63 & MM \\
\hline $12-23-94$ & 1247 & 90.90 & 58.13 & 0.02 & 416.22 & MM \\
\hline $12-23-94$ & 1247 & 90.90 & 58.13 & 0.02 & 416.22 & MM \\
\hline 01-19-95 & 1200 & 90.90 & 57.34 & 0.02 & 415.43 & $M M$ \\
\hline $04-27-95$ & 0930 & 90.86 & 59.58 & 0.02 & 497.71 & PM \\
\hline $05-01-95$ & 1236 & 90.86 & 59.27 & 0.02 & 417.40 & PMA \\
\hline
\end{tabular}


Chena River at Parks Highway Bridge

(Continued)

Site ID:

15514018 Wire Weight Gage

\begin{tabular}{|c|c|c|c|c|c|c|}
\hline Date & Tline & Check bar & WS & ErTor & WS elevation & Remarks \\
\hline $05-05-95$ & 1200 & 90.85 & 61.39 & 0.02 & 419.53 & PM \\
\hline $05-10-95$ & 1439 & 90.85 & 59.48 & 0.02 & 417.62 & PM \\
\hline $05-12-95$ & 1217 & 90.85 & 59.52 & 0.02 & 417.66 & PM \\
\hline $05-16-95$ & 1547 & 90.85 & 59.59 & 0.02 & 417.73 & MM \\
\hline $05-18-95$ & 1140 & 90.85 & 59.06 & 0.02 & 417.20 & PM \\
\hline $05-22-95$ & 1417 & 90.85 & 58.44 & 0.02 & 416.58 & PM \\
\hline $06-05-95$ & 1255 & 90.85 & 58.40 & 0.02 & 416.54 & PM \\
\hline $06-08-95$ & 1036 & 90.85 & 57.92 & 0.05 & 416.06 & PM \\
\hline $06-12-95$ & 1329 & 90.85 & 57.88 & 0.02 & 416.02 & PMA \\
\hline $06-15-95$ & 1056 & 90.86 & 58.84 & 0.02 & 416.97 & $M M$ \\
\hline $06-20-95$ & 1533 & 90.86 & 59.50 & 0.02 & 417.63 & $P M$ \\
\hline $06-28-95$ & 1635 & 90.86 & 62.90 & 0.02 & 421.03 & $P M$ \\
\hline $06-28-95$ & 1305 & 90.86 & 62.64 & 0.02 & 420.77 & PM \\
\hline $06-30-95$ & 1255 & 90.86 & 63.28 & 0.02 & $421.4 \uparrow$ & PM \\
\hline $07-03-95$ & 1136 & 90.86 & 81.81 & 0.02 & 419.94 & PM \\
\hline $07-10-95$ & 1056 & 90.86 & 61.95 & 0.02 & 420.08 & PM \\
\hline 07-13-95 & 1256 & 90.86 & 60.90 & 0.02 & 419.03 & $P M$ \\
\hline $07-17-95$ & 1522 & 90.86 & 62.59 & 0.02 & 420.72 & $M M$ \\
\hline $07-21-95$ & 1530 & 90.86 & 61.86 & 0.02 & 419.99 & PM \\
\hline $07-25-95$ & 0947 & 90.86 & 62.10 & 0.02 & 420.23 & PM \\
\hline $07-27-95$ & 1030 & 90.87 & 61.62 & 0.02 & 419.74 & PM \\
\hline $08-01-95$ & 1330 & 80.87 & 60.53 & 0.02 & 418.65 & PM \\
\hline $08-09-95$ & 1034 & 90.87 & 60.59 & 0.02 & 418.71 & PM \\
\hline $08-15-95$ & $\uparrow 620$ & 90.87 & 60.97 & 0.02 & 419.09 & MM \\
\hline $08-21-95$ & 1312 & 90.87 & 60.85 & 0.02 & 418.97 & $P M$ \\
\hline $09-15-95$ & 1643 & 90.86 & 61.17 & 0.02 & 419.30 & MM \\
\hline $10-17-95$ & NA & 90.86 & NA & 0.02 & NA & $M M$ \\
\hline
\end{tabular}




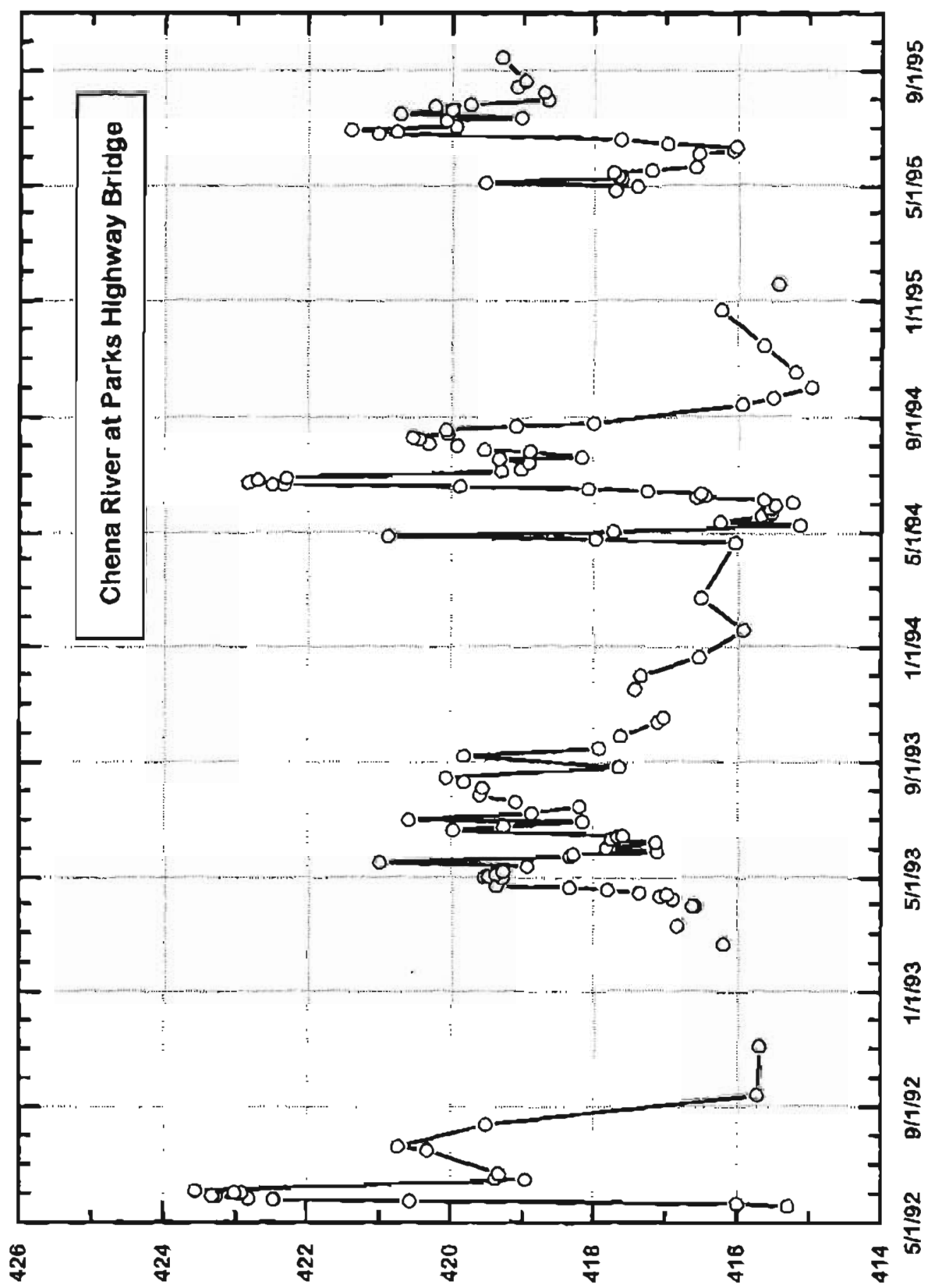

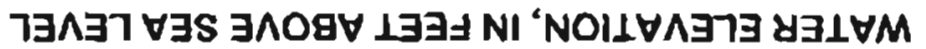

